\title{
Monitoring Radiation Damage in the ATLAS Pixel Detector
}

\author{
Dissertation \\ zur Erlangung des mathematisch-naturwissenschaftlichen Doktorgrades \\ "Doctor rerum naturalium" \\ der Georg-August-Universität Göttingen \\ im Promotionsprogramm ProPhys \\ der Georg-August University School of Science (GAUSS)
}

vorgelegt von

André Lukas Schorlemmer

aus Herdecke

Göttingen, 2014 
Betreuungsausschuss:

Prof. Dr. Arnulf Quadt, II. Physikalisches Institut, Georg-August-Universität Göttingen PD Dr. Jörn Große-Knetter, II. Physikalisches Institut, Georg-August-Universität Göttingen Dr. Beniamino Di Girolamo, Physics Department: ATLAS Detector Operation, CERN

Mitglieder der Prüfungskommission:

Referent: $\quad$ Prof. Dr. Arnulf Quadt, II. Physikalisches Institut, Georg-August-Universität Göttingen

Korreferent: PD Dr. Jörn Große-Knetter, II. Physikalisches Institut, Georg-August-Universität Göttingen

Weitere Mitglieder der Prüfungskommission:

Prof. Dr. Ariane Frey, II. Physikalisches Institut, Georg-August-Universität Göttingen

Jun.-Prof. Dr. Steffen Schumann, II. Physikalisches Institut, Georg-August-Universität Göttingen

PD Dr. Ralf Bernhard, Angehöriger der Abteilung Quadt (ATLAS), II.Physikalisches Institut, Georg-August-Universität Göttingen angestellt am Corvinianum Northeim

PD Dr. Kevin Kröninger, II. Physikalisches Institut, Georg-August-Universität Göttingen

Tag der mündlichen Prüfung: 09.07.2014 


\title{
GEORG-AUGUST-UNIVERSITÄT GÖTTINGEN \\ II. Physikalisches Institut
}

\section{Monitoring Radiation Damage in the ATLAS Pixel Detector}

by

\author{
André Lukas Schorlemmer
}

\begin{abstract}
Radiation hardness is one of the most important features of the ATLAS pixel detector in order to ensure a good performance and a long lifetime. Monitoring of radiation damage is crucial in order to assess and predict the expected performance of the detector. Key values for the assessment of radiation damage in silicon, such as the depletion voltage and depletion depth in the sensors, are measured on a regular basis during operations. This thesis summarises the monitoring program that is conducted in order to assess the impact of radiation damage and compares it to model predictions.

In addition, the physics performance of the ATLAS detector highly depends on the amount of disabled modules in the ATLAS pixel detector. A worrying amount of module failures was observed during run I. Thus it was decided to recover repairable modules during the long shutdown (LS1) by extracting the pixel detector. The impact of the module repairs and module failures on the detector performance is analysed in this thesis.
\end{abstract}





\section{Contents}

\begin{tabular}{ll}
\hline 1. Introduction & 1
\end{tabular}

1.1. The Standard Model . . . . . . . . . . . . . . . . . . . . . . . . 2

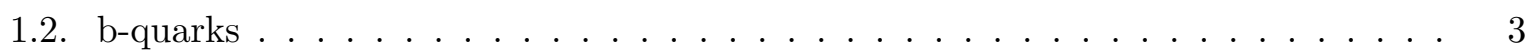

1.3. The Future of Physics at the LHC . . . . . . . . . . . . . . 4

\begin{tabular}{ll}
\hline 2. Semiconductor Detectors & 7
\end{tabular}

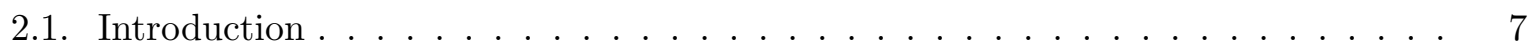

2.2. Energy Loss in Matter . . . . . . . . . . . . . . . . . . . . 8

2.3. Design of Semiconductor Sensors . . . . . . . . . . . . . . . . . . . . . . . . . 11

$2.3 .1 . \quad$ p-n Junction . . . . . . . . . . . . . . . . . . . . . . 14

2.3.2. Shockley-Ramo Theorem . . . . . . . . . . . . . . . . . . 16

2.3.3. Sensors for Pixel Detectors . . . . . . . . . . . . . . . . . 16

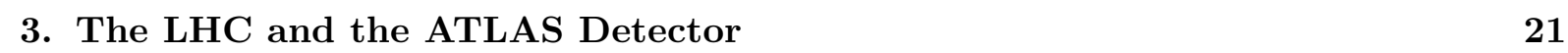

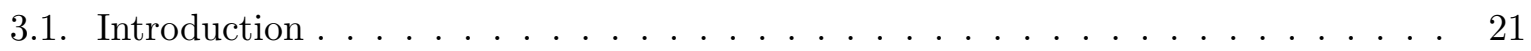

3.2. Large Hadron Collider . . . . . . . . . . . . . . . . . . . . . . . 21

3.3. ATLAS Detector . . . . . . . . . . . . . . . . . . . . . . 22

3.3.1. Coordinate System . . . . . . . . . . . . . . . . . 23

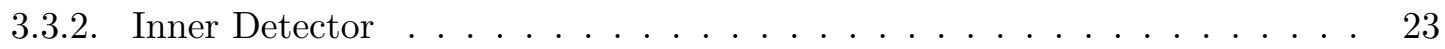

$3.3 .3 . \quad$ Calorimetry . . . . . . . . . . . . . . . . . . . . 24

3.3.4. Muon System . . . . . . . . . . . . . . . . . . . . . . 25

3.3.5. ATLAS Trigger System . . . . . . . . . . . . . . . 27

4. The ATLAS Pixel Detector 29

4.1. Introduction . . . . . . . . . . . . . . . . . . . . . . . . . 29

4.2. Layout of the Pixel Detector . . . . . . . . . . . . . . . . . . . 29

4.3. Pixel Module . . . . . . . . . . . . . . . . . . . . . . . . . . . . 30

4.3 .1$. Flex-Hybrid . . . . . . . . . . . . . . . . . . . . . . . . . . . . . . . . . . . . 31

4.3.2. The Pixel Sensor . . . . . . . . . . . . . . . . . . . . . . . 32

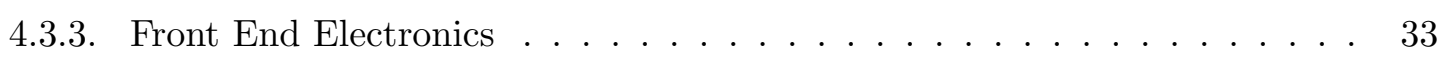

4.4. Readout System and Optoboards . . . . . . . . . . . . . . . . . . 34

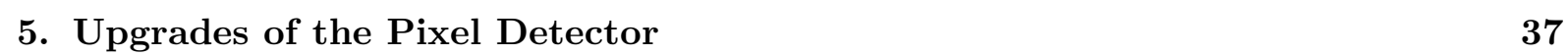

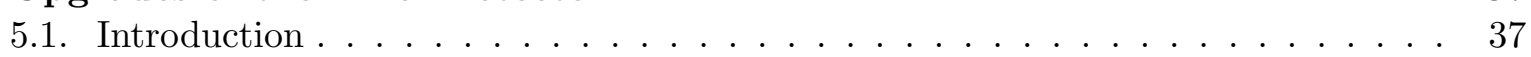

5.2. Insertable B-Layer $\ldots \ldots \ldots \ldots$. . . . . . . . . . . . . . . . . . . 37

5.2 .1$. Motivation . . . . . . . . . . . . . . . . . . 37

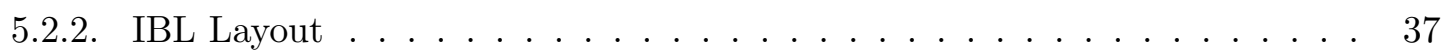

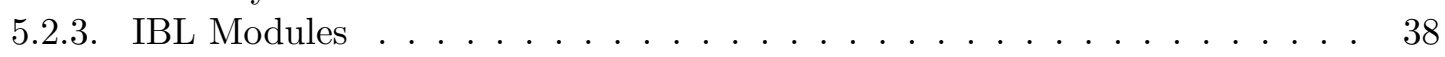

$5.3 . \quad$ New Service Quarter Panels . . . . . . . . . . . . . . . . . . . . 40

5.3 .1$. Motivation . . . . . . . . . . . . . . . . . 40

$5.3 .2 . \quad$ New Pixel Services . . . . . . . . . . . . . . . . . . . . . . 41 
6. Monitoring Radiation Damage in the Pixel Sensor 43

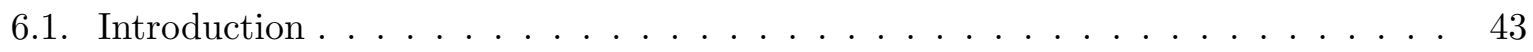

6.2. Radiation Damage in Silicon . . . . . . . . . . . . . . . . . . 44

6.2 .1$. Bulk Damage . . . . . . . . . . . . . . . . . . . . . 44

6.2 .2 . Impact on Sensor Performance . . . . . . . . . . . . . . . . . . . 47

$6.3 . \quad$ Local Coordinate System _ . . . . . . . . . . . . . . . . . . . . . 52

6.4. Measurement of the effective Depletion Voltage before type-inversion . . . . . . . 54

6.4.1. Measurement Method . . . . . . . . . . . . . . . . 54

6.4.2. Scans and Module Selection . . . . . . . . . . . . . . . . . 56

6.4.3. Evolution of the effective Depletion Voltage . . . . . . . . . . . . . . . 57

6.5. Track Position based Depletion Depth Measurement . . . . . . . . . . . . . . 63

6.5 .1$. Measurement Method . . . . . . . . . . . . . . . . . 63

6.5.2. Sources of Systematic Uncertainties . . . . . . . . . . . . . . . 70

$6.5 .3 . \quad$ Data Samples and Track Selections . . . . . . . . . . . . . . . . . . . . 71

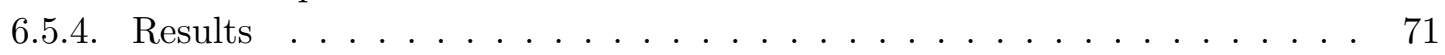

6.6. Cluster Depth based Depletion Depth Measurement . . . . . . . . . . . . . . 73

6.6.1. Measurement Method . . . . . . . . . . . . . . . . . . 73

6.6.2. Validation with Monte Carlo Simulations . . . . . . . . . . . . . . 78

6.6.3. Sources of Systematic Uncertainties . . . . . . . . . . . . . . 78

6.6.4. Geometric Constraints . . . . . . . . . . . . . . . . . . . . . 79

6.6 .5 . Data Samples and Quality Cuts. . . . . . . . . . . . . . . . . . 81

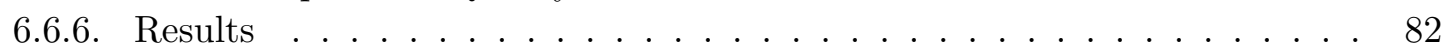

6.7. Measurement of the Effective Depletion Voltage after Type-Inversion . . . . . . . 87

6.7.1. Measurement Method . . . . . . . . . . . . . . . 87

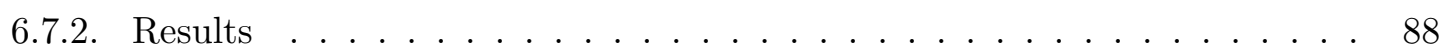

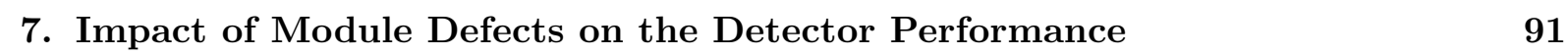

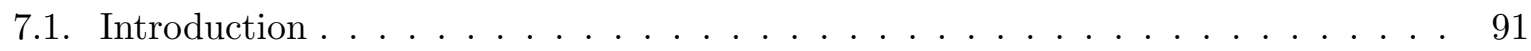

7.2. Evolution of Module Failures . . . . . . . . . . . . . . . . . . . . 91

7.3. Prediction of Module Failures $\ldots \ldots \ldots \ldots$. . . . . . . . . . . . . 92

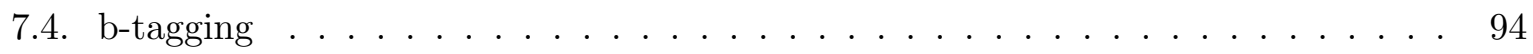

7.4.1. The Impact Parameter based Algorithm IP3D . . . . . . . . . . . . . . 95

7.4.2. The Secondary Vertex based Algorithm SV1 . . . . . . . . . . . . . . . 97

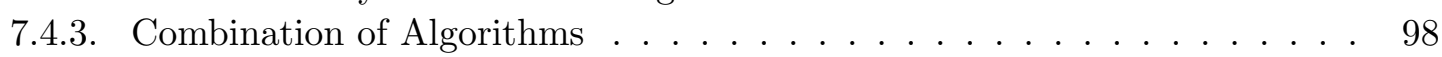

7.4.4. Efficiency and Jet Rejection . . . . . . . . . . . . . . . . . . . . . . 98

7.5. Data Samples and Selection . . . . . . . . . . . . . . . . . . . . . . . . . . . 98

7.6. Impact on Light Jet Rejection. . . . . . . . . . . . . . . . . . . . . . . . . 99

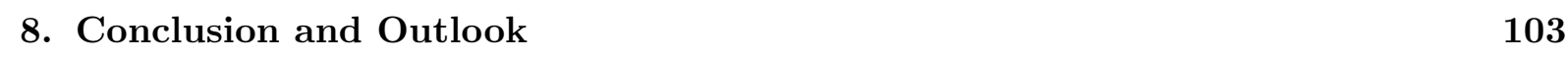

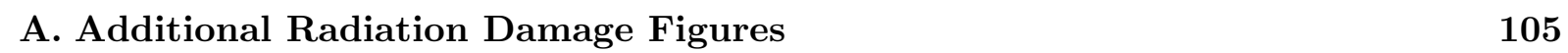

A.1. Additional Depletion Voltage Plots . . . . . . . . . . . . . . . . . . 105

A.2. Additional Cluster Depth Plots . . . . . . . . . . . . . . . . . . . . . . . 106

A.3. Additional Depletion Voltage Plots after type-inversion. . . . . . . . . . . . . 107

\begin{tabular}{ll}
\hline B. Additional Tables & 109
\end{tabular}

\begin{tabular}{ll}
\hline Bibliography & 111
\end{tabular} 
\begin{tabular}{ll}
\hline Acknowledgements & 117
\end{tabular} 



\section{Introduction}

The goal of particle physics is to describe and understand the most basic constituents of matter. Elementary particles are explained within the Standard Model, a theory that has been very precisely measured and tested. However, a number of questions still remain unanswered.

In order to further improve the knowledge of the known particles and possibly find hints on new physics beyond the Standard Model a new particle collider, The Large Hadron Collider, was built in Geneva, Switzerland. To answer some of the remaining questions in the Standard Model new very precise measurements at high energies are needed and thus new detector technologies had to be developed and built. The start up of the new accelerator in 2010 was an important milestone for particle physics, as it is currently the newest and most powerful particle collider in the world. Only after a few years of operation in 2012 a new particle was discovered, which was later confirmed to be a Higgs boson. This discovery lead to the award of the Nobel Prize in physics to François Englert and Peter Higgs in 2013, which is a great success for particle physics.

Several detectors were built together with the Large Hadron Collider for the purpose of probing the fundamental particles in our universe. One of these experiments is the ATLAS detector, which consists of several subdetectors. An important function of each experiment in high energy physics is vertex detection and the reconstruction of particle tracks. The innermost detector layer of the ATLAS detector is responsible for this task and it consists partially of pixel modules. These modules use silicon sensors as the active area for particle detection. The high energy and the high particle flux in the collider present a challenge for every detector system that is used in such an environment.

Therefore, radiation hard semiconductors have to be used in the pixel detector. Nevertheless, the highly energetic particles lead to radiation damage in the sensors. This leads to a decrease in detector performance and limits its lifetime. A good performance of the detector is crucial for new discoveries and precision measurements. In order to ensure that the damage is as large as expected it is monitored on a regular basis. The impact of radiation damage becomes more and more visible in the pixel detector. The monitoring program developed throughout the course of this thesis and the results of these measurements are presented.

Furthermore, pixel detector upgrades have been installed during the first long shutdown of the Large Hadron Collider between 2013/2014. The upgrades are designed to mitigate the impact of radiation damage and module failures in the pixel detector. The impact of the upgrades on the detector performance is studied using Monte Carlo data and the results of these studies are shown.

A short summary of the Standard Model in particle physics is given in the following Section, while a general overview of semiconductor detectors is given in Chapter 2 . The Large Hadron Collider and the ATLAS detector are described in Chapter 3 . A detailed overview of the ATLAS pixel detector is given in Chapter 4 and improvements of the current pixel detector are presented in Chapter 5. Radiation damage effects in silicon and the current monitoring program in the pixel detector are described in Chapter 6. The impact of module failures on the detector performance is explained in Chapter 7 and a final conclusion is given in Chapter 8 . 


\subsection{The Standard Model}

The Standard Model (SM) describes the fundamental components of matter as particles and three of the four known fundamental forces between them. The most basic constituents of matter are described as particles with a half-integer spin (fermions) and there are two types of elementary fermions, quarks and leptons. Leptons are summarised in Table 1.1 and quarks are summarised in Table 1.2 ,

The electrical charge of all particles is shown in units of the electron charge (e). Leptons have either a charge that is zero or equal to the electron charge, while the charge of a single quark is a rational number with an absolute value smaller than the electron charge. Six leptons and six quarks exist and for each particle there is an anti-particle with opposite quantum numbers (charge, colour, etc.). Quarks and leptons are combined into generations depending on their physical properties.

\begin{tabular}{|c|c|c|c|}
\hline Generation & Particle & Mass & Charge $(\mathrm{e})$ \\
\hline 1 & $e$-neutrino $\left(\nu_{e}\right)$ & $<2 \mathrm{eV}$ & 0 \\
& electron $(e)$ & $0.511 \mathrm{MeV}$ & -1 \\
\hline 2 & $\mu$-neutrino $\left(\nu_{\mu}\right)$ & $<0.19 \mathrm{MeV}$ & 0 \\
& muon $(\mu)$ & $105.7 \mathrm{MeV}$ & -1 \\
\hline 3 & $\tau$-neutrino $\left(\nu_{\tau}\right)$ & $<18.2 \mathrm{MeV}$ & 0 \\
& $\operatorname{tau}(\tau)$ & $1.777 \mathrm{GeV}$ & -1 \\
\hline
\end{tabular}

Table 1.1.: Leptons in the Standard Model and their properties [1].

\begin{tabular}{|c|c|c|c|}
\hline Generation & Particle & Mass & Charge (e) \\
\hline 1 & up $(u)$ & $1.8-3 \mathrm{MeV}$ & $+\frac{2}{3}$ \\
& down $(d)$ & $4.5-5.3 \mathrm{MeV}$ & $-\frac{1}{3}$ \\
\hline 2 & charm $(c)$ & $1.27 \mathrm{GeV}$ & $+\frac{2}{3}$ \\
& strange $(s)$ & $95 \mathrm{MeV}$ & $-\frac{1}{3}$ \\
\hline 3 & top $(t)$ & $173 \mathrm{GeV}$ & $+\frac{2}{3}$ \\
& bottom $(b)$ & $4.2 \mathrm{GeV}$ & $-\frac{1}{3}$ \\
\hline
\end{tabular}

Table 1.2.: The quarks and their properties 11 .

There are four known fundamental forces in nature, three of them are described by the Standard Model. Interactions between particles are described by exchange particles with integer spin (bosons). The gauge bosons and the corresponding forces are summarised in Table 1.3.

Quarks carry colour charge and thus interact strongly, while gluons are responsible for the strong interaction between the quarks. Gluons also carry colour charge and thus interact strongly with each other, which leads to the short range of the strong interaction and explains why quarks are confined.

The carrier of the electromagnetic interaction is the photon. Like the gluon it has no mass, which leads to an infinite range of the electromagnetic interaction. Weak interactions are carried out by the electrically charged $W$ bosons and the neutral $Z$ boson. The range of the weak force is limited due to the large mass of the gauge bosons. At higher energies the electric and the weak interactions are combined into the electroweak interaction [2, 3, 4].

The Standard Model can not describe several phenomena. One of the most interesting questions is the origin of mass of elementary particles. A very promising extension to the Standard 


\begin{tabular}{|c|c|c|}
\hline Interaction & Particles & Mass \\
\hline strong & 8 gluons $(g)$ & 0 \\
electromagnetic & photon $(\gamma)$ & $<1 \cdot 10^{-18} \mathrm{eV}$ \\
weak & $W$-Bosons $\left(W^{ \pm}\right)$ & $80.4 \mathrm{GeV}$ \\
weak & $Z$-Boson $\left(Z^{0}\right)$ & $91.2 \mathrm{GeV}$ \\
\hline
\end{tabular}

Table 1.3.: Interactions and the associated particles in the Standard Model [1].

Model is the Higgs mechanism [5, 6, 77. In electroweak theory the $W$ and $Z$ bosons need to be massless. Using the Higgs mechanism heavy masses of the bosons are created by a coupling between the bosons and a scalar background field. In addition, it is also possible to explain the masses of other particles in the Standard Model with this mechanism. One consequence of the Higgs theory is the postulation of a new spinless particle, the Higgs boson.

The recent discovery of a new boson with an approximate mass of $125 \mathrm{GeV}$ at the LHC [8, 9$]$ is an important milestone for particle physics [1]. The recently observed properties of the boson strongly indicate that the particle is a Higgs boson. However, further measurements are needed in order to confirm if the boson is the Standard Model Higgs boson, or if the observed Higgs particle shows properties that can only be explained by models beyond the Standard Model.

\section{2. b-quarks}

Understanding the production of b-quarks is important for studies of QCD predictions, thus, a short and non extensive summary of the production and decay mechanisms is given in the following. Examples of Feynman diagrams demonstrating some of the dominating b-quark production modes in hadron colliders are shown in Figure 1.1. In these cases b-quarks are produced as quark-antiquark pairs. Only the leading order diagrams are shown for demonstration. Additional information on heavy quark production as well as higher order processes can for example be found in 10,11 .
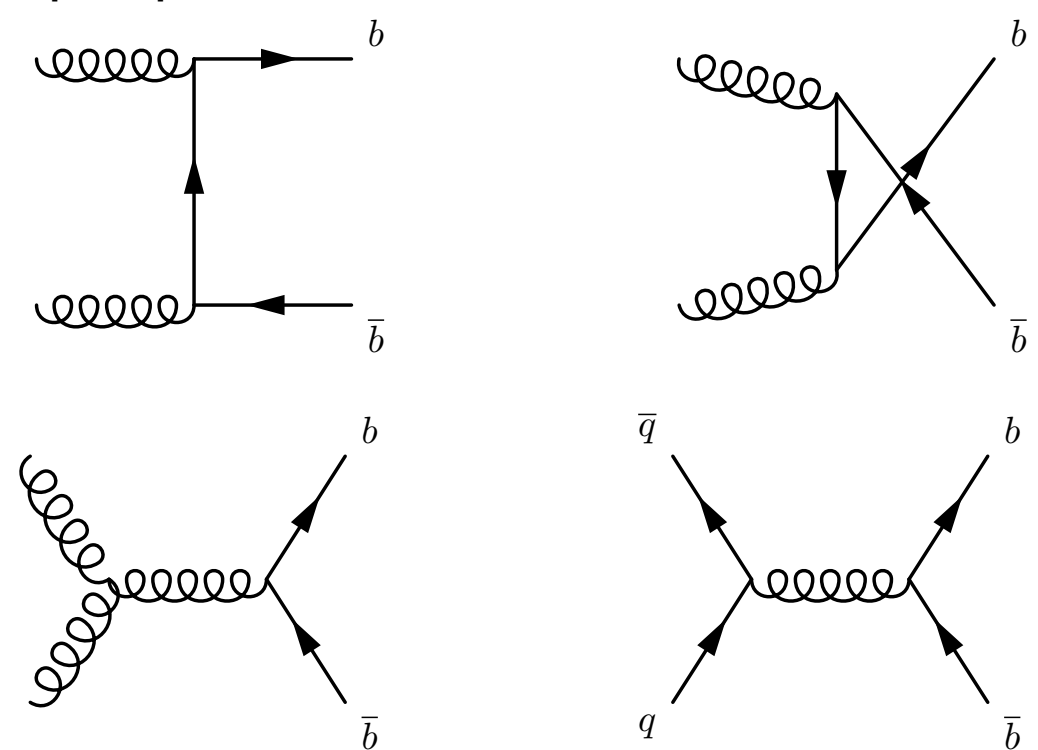

Figure 1.1.: Feynman diagrams demonstrating the production of b-quarks via gluon gluon fusion and quark-antiquark annihilation 10 .

Important signatures of physics processes that are studied at the LHC also often contain a 
b-quark. For precise studies of these processes it is important to identify the b-quarks that are produced in the decay chain and many studies exploit the fact of this identification. For example top quark and Higgs boson properties are of great interest and b-quarks are often produced during their decay. Two Feynman diagrams of the most common top and Higgs decay modes are shown in Figure 1.2 .
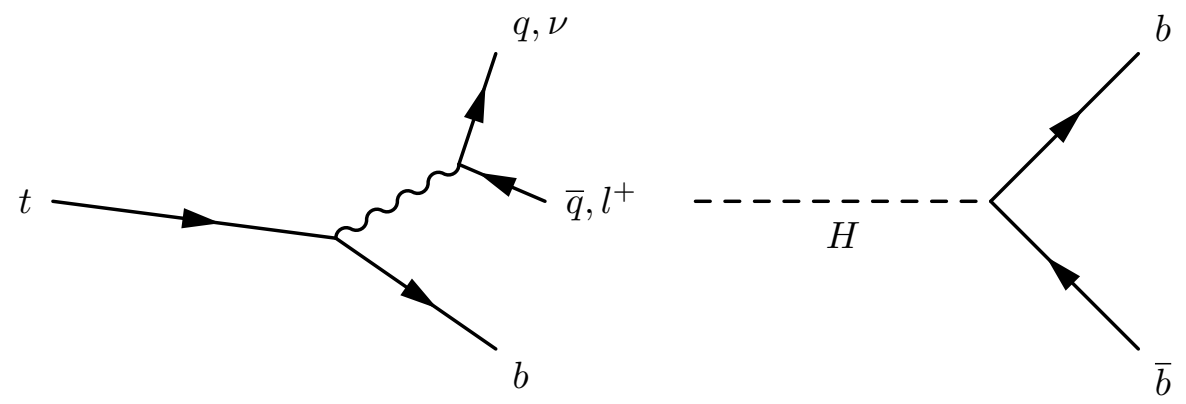

Figure 1.2.: Physics processes involving b-quarks. An example for the decay of the top quark is shown of the left, while an example of the Higgs decay is shown on the right.

b-quarks hadronise into mesons, a pair of a quark and an anti-quark as well as baryons, a composition of three quarks. A list of particles containing b-quarks can be found in [1]. During the hadronisation the b-hadron keeps in general most of the momentum of the b-quark unlike most other hadrons, which are only composed of lighter quarks. The fraction of the b-quark momentum that is transmitted to the newly formed b-hadron is described by the fragmentation function [1, 12].

According to [1] ground state b-hadrons decay weakly, which is described in the spectator model. The dominant mode of decay is $b \rightarrow c W^{*-}$ and the virtual $W^{*-}$ boson decays into a pair of leptons or hadrons. An example of a b-hadron decay is shown in Figure 1.3. The mean lifetime for the b-hadron mixture is $1.568 \pm 0.009 \mathrm{ps}$ [1] and the difference in the lifetime for the different b-hadrons is relatively small. Since b-hadrons keep most of their momentum during hadronisation they can fly a few millimetres before they decay. This displacement from the primary interaction point offers the opportunity to identify b-jets.

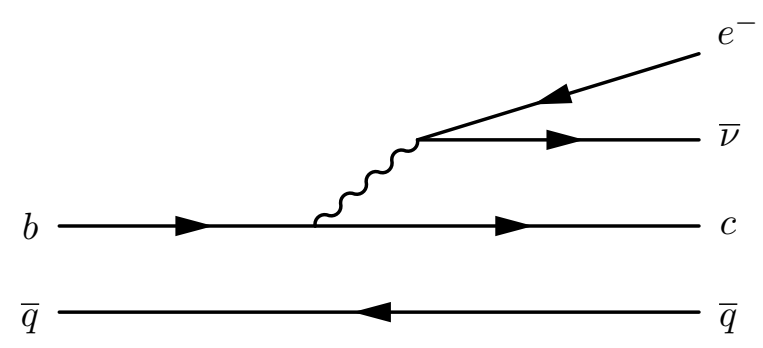

Figure 1.3.: Example of b-hadron decay 11$]$.

\subsection{The Future of Physics at the LHC}

In order to further improve the potential for the discovery of new physics in the Large Hadron Collider (LHC). It is planned to upgrade the detectors and the accelerator ring during several shutdown cycles. A major upgrade of the LHC is planned around 2020, with the goal of increasing the number of collisions and collecting a total luminosity of up to $3000 f b^{-1}[13$. 
After the discovery of the Higgs boson and with growing statistics in the future it will be possible to focus on more detailed measurements of its properties. A detailed discussion of the following short summary of the Higgs and weak boson scattering measurement program is presented in [14. Not all properties of the Higgs boson have been measured precisely yet and several models such as supersymmetry predict different couplings than the Standard Model Higgs would posses. Thus, precise measurements of the Higgs coupling are very interesting in order to test the Standard Model further and in the search for new physics. Furthermore, the Standard Model Higgs couples to itself, thus, for a full investigation of its properties it is necessary to measure the Higgs self coupling. This could be achieved by using the Higgs boson pair production at the LHC.

The cross section of vector boson scattering would rise infinitely without the Higgs mechanism at the $\mathrm{TeV}$ scale. Other models that might lead to the cross section limitation have been proposed and therefore measurements of the vector boson scattering cross section are interesting since they could lead to new physics.

Furthermore, gravity is not explained in the frame of the Standard Model and only a fraction of the universe is composed of the known constituents. A large part of the universe consists of the so called dark matter and dark energy, which are not included in the current theory. Some of the most popular explanations for these questions are the already mentioned supersymmetric theories. Supersymmetric models predict several new particles and many of them are expected to have b-quarks in their decay chains. In order to be able to discover phenomena beyond the Standard Model it is thus very important for detectors to identify particle jets coming from b-quarks as reliably as possible. A more extensive discussion of the search for supersymmetric models and additional topics such as exotic resonances can be found in [13]. 



\section{Semiconductor Detectors}

\subsection{Introduction}

In general, semiconductor detectors consist of a sensor where the signal is produced and the readout electronics, where the induced signal is processed. Particles passing through matter can be detected due to the fact that they ionise the atoms in the sensor and thus create electron hole pairs. The created charge carriers are separated in the presence of an electric field. They travel towards the readout electronics, which is designed to measure the electric pulse of each charged particle passing the detector.

The most widely used material in semiconductor detectors is silicon it is well developed, used in various applications and available for a relatively low prize. Additional materials that are also frequently used in semiconductor detectors as sensors are germanium and diamond.

Semiconductors have specific properties that makes them especially suitable for particle detection and they are explained using silicon detectors as an example. According to [15] silicon detectors have the following advantages compared to detectors that use gas:

- The band gap of $1.12 \mathrm{eV}$ in silicon is relatively small, while the average energy of creating an electron whole pair is $3.6 \mathrm{eV}$. Gas detectors have an ionisation energy of about $30 \mathrm{eV}$. Thus, many charge carriers are created per unit energy loss if a charged particle passes a silicon sensor.

- Silicon is a high density material $\left(\rho=2.23 \frac{\mathrm{g}}{\mathrm{cm}^{3}}\right)$. This leads to a large amount of lost energy per travelled length segment of the passing particle. This offers the opportunity to build very thin sensors that deliver a sufficiently large signal.

- Electrons and holes have a very high mobility within the silicon sensor. The mobilities are $\mu_{n}=1450 \frac{\mathrm{cm}^{2}}{\mathrm{Vs}}$ for electrons and $\mu_{p}=450 \frac{\mathrm{cm}^{2}}{\mathrm{Vs}}$ for holes. This leads to a very fast charge collection.

- The high mechanical rigidity of silicon offers the opportunity to design structures that are self-supporting.

- A large improvement over gas detectors is the option to dope parts of the silicon sensor, thus creating a space charge region. This allows the creation of a sophisticated field configuration without interfering with the movement of signal charges.

- The sensor and the electronics can be joined in one single device, due to the fact that both parts can be manufactured out of silicon.

In gas detectors it is often necessary to find a balance between readout speed and precision depending on the requirements. Gas detectors that are used for position measurements are thus limited by their readout speed. Drift chambers for example, are limited in their precision by the drift time of the free charge carriers, while pixel detectors can be fast and very precise, which makes them better suited for position measurements in high occupancy environments. 
A short summary of basic interactions of particles passing through matter is given in Section 2.2. A special emphasis is given to the reactions that are important for particle detection in high energy physics. General principles of silicon detectors and their design are described in Section 2.3 .

\subsection{Energy Loss in Matter}

Charged particles lose part of their energy if they pass through matter. Particles with sufficient energy and a mass larger than the electron mass lose their energy primarily by ionisation and excitation of electrons, which is the desired behaviour in particle sensors. Additional interactions of highly energetic particles also lead to non ionising energy losses, which have a negative impact on the sensor performance. This Chapter focuses on the general concept of silicon detectors and therefore, only ionising energy losses are discussed in the following, while the additional processes are presented in Chapter 6 .

The average energy loss per distance (stopping power) is given by the Bethe-Bloch equation [16]:

$$
-\frac{\mathrm{d} E}{\mathrm{~d} x}=2 \pi N_{0} r_{e}^{2} m_{e} c^{2} \rho \frac{Z}{A} \frac{z^{2}}{\beta^{2}}\left[\ln \left(\frac{2 m_{e} \gamma^{2} v^{2} W_{\max }}{I^{2}}\right)-2 \beta^{2}-\delta-2 \frac{C}{Z}\right]
$$

with the following values:

Avogadro's number:

Classical electron radius:

Electron mass:

Speed of light:

Density of the material:

Atomic number of the material:

Atomic weight of the material:

Charge in units of $e$ :

Ratio of the velocity $v$ to the speed of light $c$ :

Lorentz factor:

Maximum energy transfer in a single collision:

Mean excitation potential:

Density correction:

Shell correction:

$$
\begin{aligned}
& N_{0}=6.022 \cdot 10^{23} \mathrm{~mol}^{-1}, \\
& r_{e}=2.817 \cdot 10^{-13} \mathrm{~cm}, \\
& m_{e}, \\
& c, \\
& \rho, \\
& Z, \\
& A, \\
& z, \\
& \beta=\frac{v}{c}, \\
& \gamma=\frac{1}{\sqrt{1-\beta^{2}}}, \\
&
\end{aligned}
$$

$W_{\max }$,

$I$,

$\delta$,

C.

The maximum energy transfer is:

$$
W_{\max }=\frac{2 m_{e} c^{2} \beta^{2} \gamma^{2}}{1+2 \frac{m_{e}}{M} \sqrt{1+\beta^{2} \gamma^{2}}+\frac{m_{e}^{2}}{M^{2}}} .
$$

In case of $M \gg m_{e}$, the formula reduces to:

$$
W_{\text {max }} \approx 2 m_{e} c^{2} \beta^{2} \gamma^{2} .
$$

The density correction is necessary because the electric field of the particles tends to polarise the atoms along its track. This polarisation shields electrons further away from the particle track and collisions with outer electrons will thus contribute less to the energy loss than initially 
predicted by the formula. The magnitude of the effect depends on the density of the material and the correction becomes larger with increasing particle energy.

The approximation in the Bethe-Bloch formula that the electron is stationary with regard to the passing particle is not valid if the velocity of the passing particle is similar to the velocity of the bound electrons. In order to correct the formula, the shell correction term is added.

An example of the stopping power as a function of the muon momentum is shown in Figure 2.1. The Bethe-Bloch formula is only valid at moderate energies. At lower energies the Bethe-Bloch formula is applicable to a $\beta \approx 0.05$ with a precision of $\approx 1 \%$ due to the shell correction, while no adequate theory exist for $0.01<\beta<0.05[1]$. At very high energies radiative energy losses play a key role, which are not described by the Bethe-Bloch formula. In iron the critical point for radiative losses is found at several hundred $\mathrm{GeV}$ for muons and pions, while it is significantly higher for protons. Particles passing through matter with energies at which the stopping power is minimal are called minimum ionising particles. The increase of the stopping power after the minimum is relatively small as visible in the figure. Thus, in particle physics traversing particles are often approximated as minimum ionising particles.

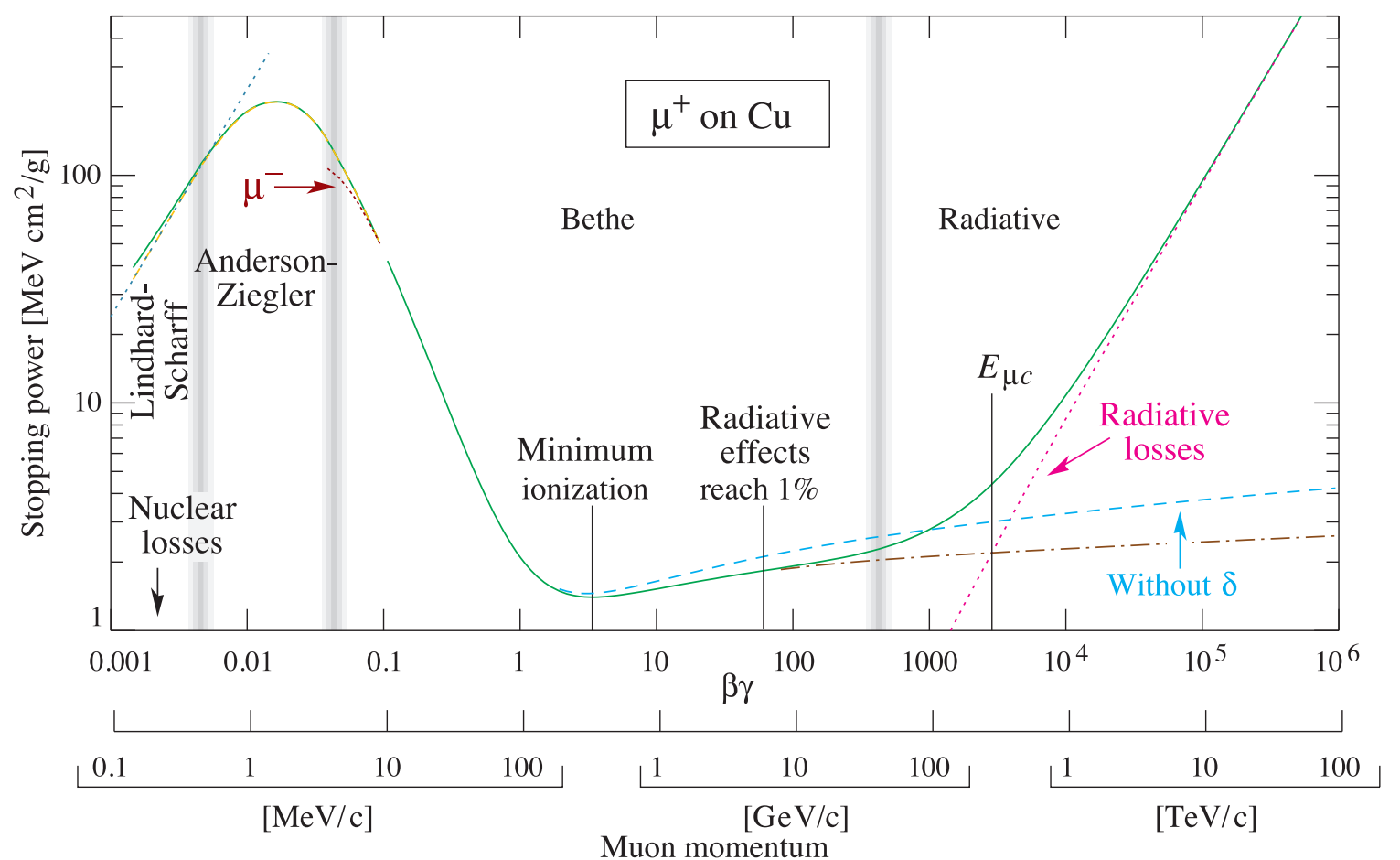

Figure 2.1.: Stopping power $\frac{\mathrm{d} E}{\mathrm{~d} x}$ as a function of the momentum. Positive muons in copper are shown as an example [1].

Only the mean energy loss is described by the Bethe-Bloch formula, while the energy loss of a single particle can fluctuate drastically. The mean value and the most probable value are not identical because the shape of the energy loss distribution is asymmetric. The most probable energy loss is described by Landau [1, 17]:

$$
\Delta_{p}=\xi\left[\ln \frac{2 m c^{2} \beta^{2} \gamma^{2}}{I}+\ln \frac{\xi}{I}+0.200-\beta^{2}-\delta(\beta \gamma)\right] .
$$

with a sensor thickness $x$ in $\frac{g}{c m^{2}}$ and $\xi=\frac{K}{2}\left\langle\frac{Z}{A}\right\rangle \frac{x}{\beta^{2}} \mathrm{MeV}$. The energy loss distribution has been described by Landau, Varilov, and Symon [18, 19]. All three theories have a different range of 
application and they describe the energy loss distributions well as long as the sensors are not too thin.

For silicon detectors the Landau distribution is usually the best approximation. However, for very thin silicon sensors the width of the energy loss distribution is much wider than the width of the Landau distribution. Even so, the most probable value can still be approximated by Equation 2.4 .

Examples of straggling functions in silicon are given in Figure 2.2. The functions are normalised to unity at the most probable value and the full width at half maximum, $w$, is indicated. It is clearly visible that the most probable value depends on the thickness of the sensor, while the mean value is constant. Over $90 \%$ of the interactions lead to an energy loss that is smaller than the mean loss, while the asymmetric tail is only caused by a small amount of collisions with a high energy transfer [1]. The large tail is mainly created by $\delta$-electrons which have received enough energy in the collision to ionise additional electrons. Various theoretical descriptions have been developed in order to characterise straggling functions in thin silicon sensors. Additional information on this topic can be found in 17,20 .

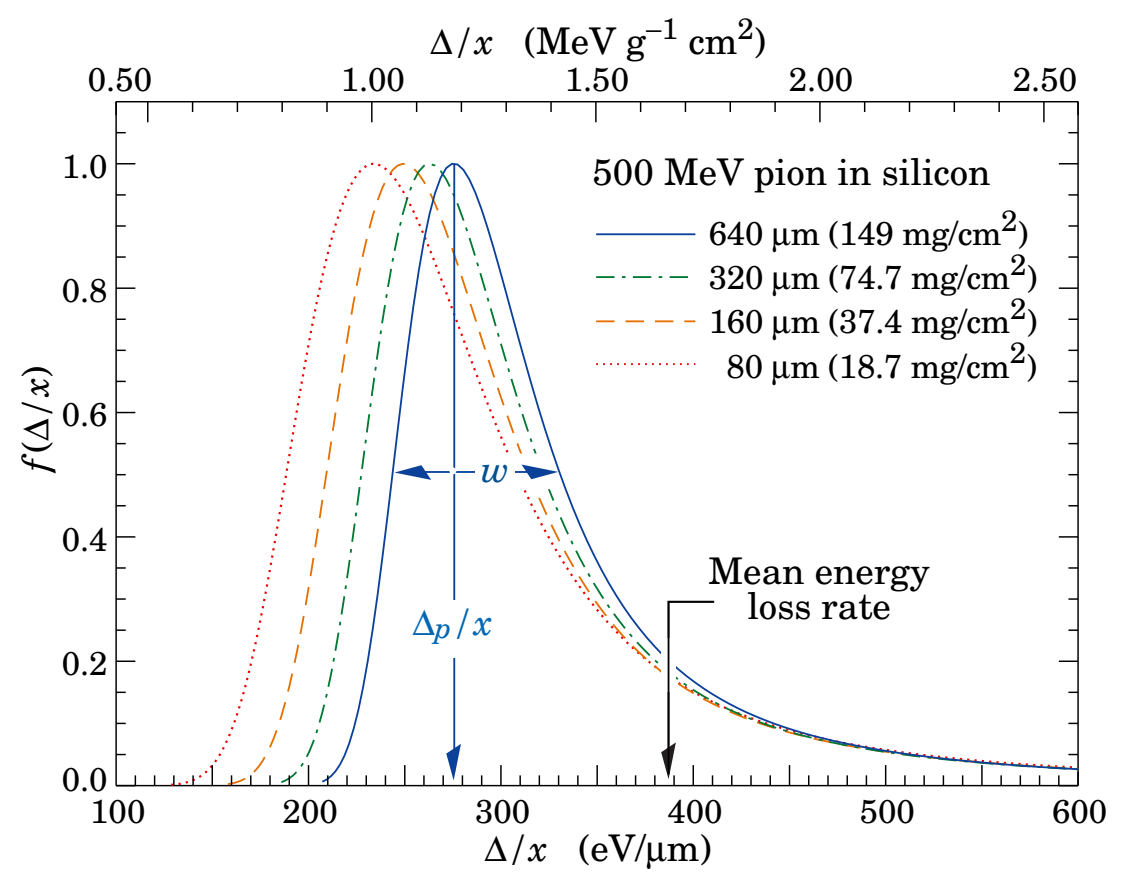

Figure 2.2.: Straggling function in silicon shown for $500 \mathrm{MeV}$ pions and a varying sensor thickness [1].

The energy loss of electrons is slightly different compared to that of heavier charged particles. Radiative losses always need to be regarded, because of the relatively small electron mass. Thus, the energy loss of electrons is always a combination of collisional and radiative energy losses [16]:

$$
\left(\frac{d E}{d x}\right)_{\text {tot }}=\left(\frac{d E}{d x}\right)_{\mathrm{rad}}+\left(\frac{d E}{d x}\right)_{\mathrm{coll}}
$$

The radiation losses are created by the deflection of the electron in the electric field of a nucleus (Bremsstrahlung). The effect is still relatively small for electrons with energies of a magnitude of $1 \mathrm{MeV}$. The probability of radiative losses rises quickly with the electron energy and it is 
similar or larger than the probability for collision losses around a magnitude of $10 \mathrm{MeV}$ [16]. At particle energies below a few hundred $\mathrm{GeV}$, electrons and positrons are the only particles where radiative losses are an important factor.

Collision losses for electrons are in general similar to collision losses of heavier particles, however, the Bethe-Bloch formula (Equation 2.1) needs to be modified. First the electron mass is small and thus it might be deflected and second, collisions take place between identical and indistinguishable particles. The modified formula can be found in [16].

\subsection{Design of Semiconductor Sensors}

A short summary of the principles of semiconductor devices with a focus on applications in pixel detectors is given in the following. An excellent and extensive explanation of semiconductor devices can be found in [21], while semiconductor detectors focused on applications in high energy physics are explained in detail in 15,22 .

In a crystal lattice all allowed energy levels of electrons are arranged in bands. The difference between insulators and conductors is the size of the gap betweens the bands. A gap between bands is a "forbidden zone", because no states exist for these energies. The highest filled band is called the valence band and the lowest empty or partially filled band is the conduction band. The size of the band gap between these two bands depends highly on the studied material. Conductors are materials where the valence band is only partially filled or where the size of the gap is zero, which allows electrons to move within the material. In insulators the band gap is in general large and electrons are bound to their nuclei at moderate temperatures.

Semiconductors have a small band gap. As mentioned, for silicon it has a size of $1.12 \mathrm{eV}[21$, which means that electrons can be lifted into the conduction band by thermal excitation. A semiconductor is called intrinsic if the amount of impurity atoms in its crystal is very small. In this case the probability of electrons occupying the conduction band is given by the Fermi-Dirac equation:

$$
F(E)=\frac{1}{1+\exp \left(\frac{E-E_{F}}{k T}\right)},
$$

where $T$ is the absolute temperature in Kelvin, $k$ is the Boltzmann constant and $E_{F}$ is the Fermi energy. At this energy, the occupation probability is exactly 0.5. The number of free electrons per unit volume $n$ in a semiconductor can be calculated by integrating the product $N(E) F(E)$ over the energy $d E . N(E)$ is the density and the interval ranges from the bottom to the top of the conduction band. The calculation is explained in detail [21], with a result of:

$$
n=N_{C} \exp \left(\frac{-\left(E_{C}-E_{F}\right)}{k T}\right)
$$

for the free electron density, where $N_{C}$ is the effective density of states in the conduction band and $E_{C}$ is the minimum energy of the conduction band. For silicon, the density of states is:

$$
N_{C}=12\left(\frac{2 \pi m_{n} k T}{h^{2}}\right)^{3 / 2}
$$

The effective mass of the electron is $m_{n}$ and $h$ is the Planck constant. A similar description is used for the free hole density:

$$
p=N_{V} \exp \left(\frac{-\left(E_{F}-E_{V}\right)}{k T}\right)
$$


$E_{V}$ is the energy at the top of the valence band and $N_{V}$ is called the effective density of states in the valence band. Silicon has a density in the valence band of:

$$
N_{V}=2\left(\frac{2 \pi m_{p} k T}{h^{2}}\right)^{3 / 2}
$$

where $m_{p}$ is the effective mass of the holes in the semiconductor. The electron concentration in an intrinsic semiconductor is equal to the hole concentration: $n=p=n_{i}$. This relationship changes if doped material is used. However, the product $n p$ of both concentrations at thermal equilibrium is independent of the Fermi level and thus $n p$ is also independent of the doping concentration:

$$
n_{i}^{2}=n p=N_{C} N_{V} \exp \left(\frac{-\left(E_{C}-E_{V}\right)}{k T}\right)
$$

For silicon, $n_{i}$ has a value of $9.65 \cdot 10^{9} \mathrm{~cm}^{-3}$ at room temperature 21, 23. The properties of a semiconductor can be changed by inserting foreign atoms into its lattice. Doped semiconductors are called extrinsic due to the large amount of foreign atoms. A schematic view of the energy band of extrinsic semiconductors is shown in Figure 2.3. Atoms with one more valence electron than the semiconductor material are called donors (n-doped). The energy level of donors is close to the conduction band. Due to small ionisation energies for donors, the additional electron is lifted into the conduction band which leads to a high concentration of free electrons in the semiconductor. Donor atoms in silicon need an energy of approximately $0.03 \mathrm{eV}$ to be ionised [21].

Acceptor atoms have one valence electron less than the semiconductor and their energy level is close to the level of the valence band. Thus, electrons are attracted by the impurities and a hole is left in the valence band. The holes are treated effectively as particle with a positive charge. Holes are mobile at moderate temperatures and thus can act as charge carriers. Normally, there is enough thermal energy available in silicon sensors to ionise all donors and acceptors in doped silicon detectors. Thus, the number of free charge carriers is $n=N_{D}$ in n-doped material and $p=N_{A}$ in p-doped semiconductors 21].
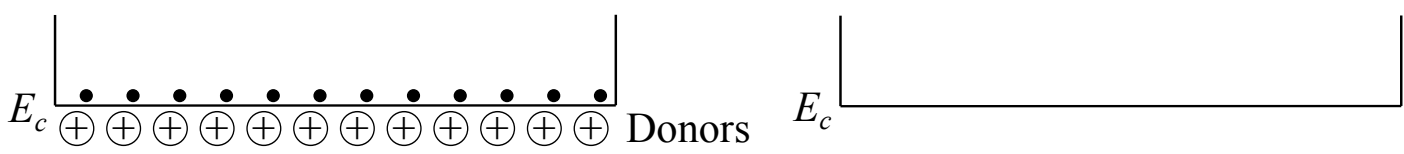

$E_{i}$
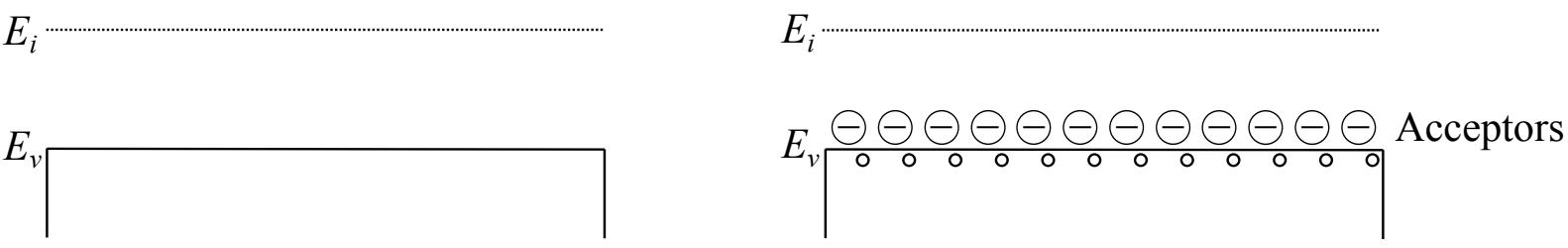

Figure 2.3.: Illustration of the energy bands in doped semiconductors. An n-doped sensor is shown on the left, while p-doped material is shown on the right. The Fermi level for an intrinsic semiconductor $E_{i}$ is indicated in both diagrams.

Drift and diffusion of charge carriers are two important factors that cause the movement of free charge carriers in semiconductors. An external electric field leads to a drift of the charge carriers in the semiconductor. The direction is defined by the field lines and the overall drift 
velocity of electrons $v_{n}$ and holes $v_{p}$ depends on the strength of the electric field $E$ and the mobility $\mu_{n, p}$ of the charge carrier 21]:

$$
\begin{aligned}
& v_{n}=-\mu_{n} E, \\
& v_{p}=\mu_{p} E .
\end{aligned}
$$

The mobility of holes in a semiconductor is in general smaller than the mobility of electrons. Both mobilities are a function of the mean free time of the charge carriers, which of course depends on the different types of scattering mechanisms that can take place in the semiconductor. Thus, the mobility is a function of the temperature and amount of impurities present in the sensor. In addition the drift velocity is only proportional to the electric field for small field strengths. At high electric fields the increase of the acceleration is counteracted by the large amount of collisions per time and the gain of the drift velocities $v_{n}$ and $v_{p}$ is no longer linear. The increase in velocity for charge carriers slows down and converges towards the corresponding saturation values for electrons and holes.

The displacement of free charge carriers due to thermal movement is random and overall zero. However, if there is a concentration difference in the charge carrier density the free electrons and holes begin to diffuse. On average charge carriers diffuse from a region with a large density into the one with smaller density.

The total current density $J$ in the semiconductor is given by the sum of the drift and diffusion components. If the carrier concentration changes in x-direction and an external electric field $E$ in x-direction is present, then the current density equations for electrons $J_{n}$ and holes $J_{p}$ are written as 21:

$$
\begin{aligned}
& J_{n}=e \mu_{n} n E+e D_{n} \frac{d n}{d x}, \\
& J_{p}=e \mu_{p} n E-e D_{p} \frac{d p}{d x},
\end{aligned}
$$

where $D_{n, p}$ is the Einstein relation which describes the dependence between the diffusion parameter and drift mobility of charge carriers 21:

$$
D_{n, p}=\left(\frac{k T}{e}\right) \mu_{n, p} .
$$

Silicon detectors are often used in the presence of a magnetic field in order to allow measurements of the particle momentum. However, the magnetic field influences not only the particle that is passing through the sensor but also the free charge carriers moving inside the silicon sensor. Thus created electron hole pairs drifting towards the electrodes in the presence of an electric field are deflected by the Lorentz force. The change in drift direction is in general described by the opening angle $\Theta_{L}$ measured with respect to the direction of the electric field. Typical values of the so called Lorentz angle lie between a few and $20^{\circ}$ and the equations for electrons $\Theta_{L, n}$ and holes $\Theta_{L, p}$ are [22]:

$$
\begin{aligned}
& \tan \Theta_{L, n}=\mu_{H, n} B_{\perp}, \\
& \tan \Theta_{L, p}=\mu_{H, p} B_{\perp},
\end{aligned}
$$

where $\mu_{H}$ is the hall mobility and $B_{\perp}$ is the magnetic field component that is perpendicular to the direction of the charge carrier drift. The hall mobility can be derived from the drift 
mobility: $\mu_{H}=r \mu$. The Lorentz factor $r$ is slightly temperature dependent and its value is 1.15 for electrons and 0.72 for holes at $0^{\circ} 22$.

\subsection{1. p-n Junction}

By connecting $\mathrm{p}$ and $\mathrm{n}$ doped material, a $\mathrm{p}-\mathrm{n}$ diode is created, which is the general structure that is used for semiconductor sensors. The general principle of semiconductor p-n junctions is illustrated in Figure 2.4. In the n-doped part the majority carrier are electrons, while holes dominate the p-type region. In the region where both types are joined, charge carriers diffuse across the junction due to the concentration difference. Electrons diffuse into the p-doped side, while holes diffuse into the n-doped semiconductor.

The diffusion leads to a recombination of electrons and holes in close proximity of the p-n junction, which creates a zone that is depleted of free charge carriers (depleted region). As a result, a negative space charge is created in the p-side of the sensor, while a positive charge is formed in the n-doped region. The space charge leads to an electric field oriented in opposite direction of the carriers diffusion. This effect prevents further diffusion of charge carriers into the area around the junction. As soon as thermal equilibrium is reached, the values of the electron and hole current at the junction decreases to exactly zero and the Fermi level is constant in the whole diode.
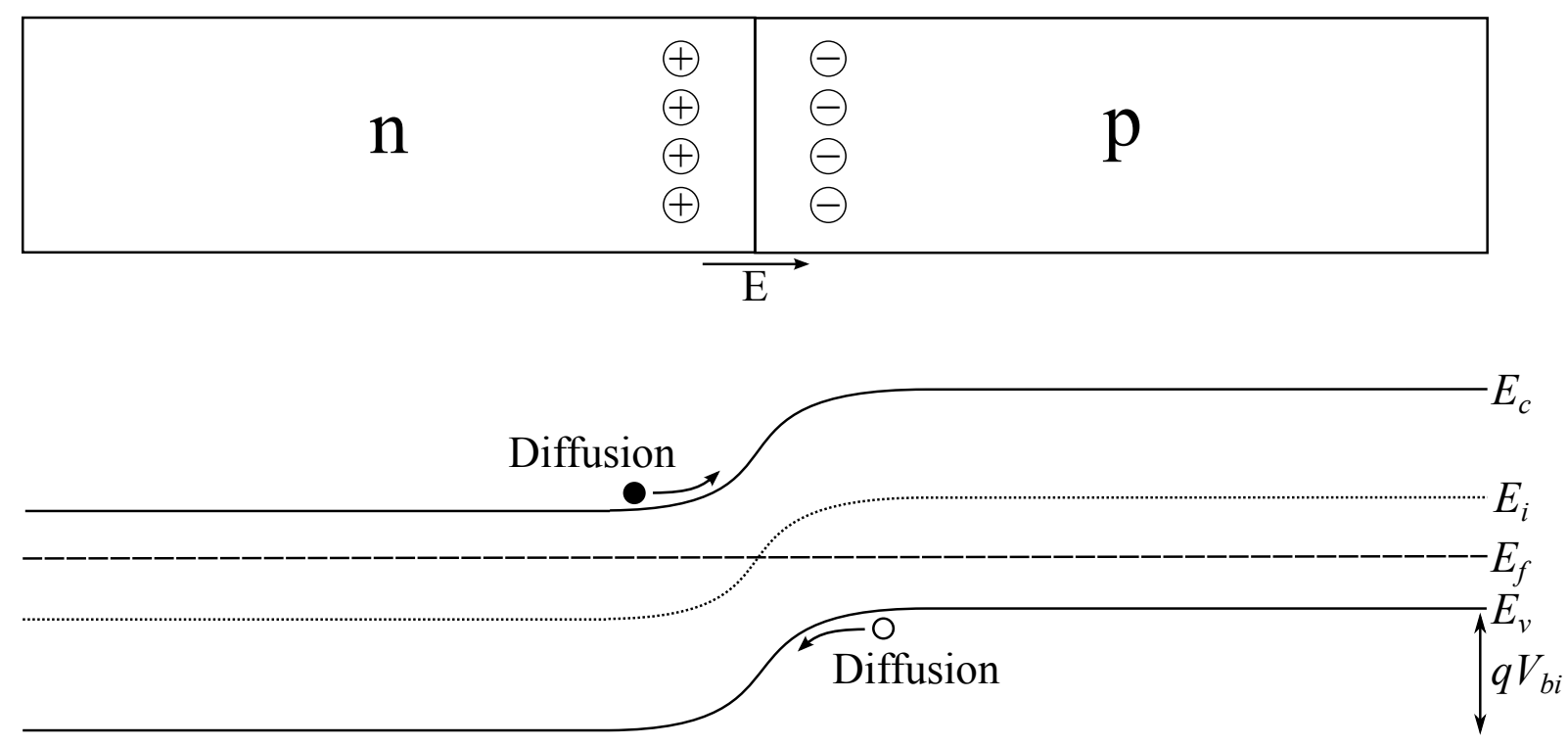

Figure 2.4.: Schematic view of a p-n junction. Charge carriers diffuse through the junction and recombine, due to the large concentration difference of electrons and holes at the junction, which leads to the creation of an electric field. The field is shown for the case of thermal equilibrium.

The built-in voltage $V_{\mathrm{bi}}$ of the junction is the potential difference between the p-side and the n-side at thermal equilibrium. The size of the depleted region $D$ that surrounds the junction is a function of $V_{\mathrm{bi}}$. Both values can be derived by solving the Poisson equation in the p-n junction. The reader is referred to the previously mentioned literature 21 for a derivation of the following equations. Assuming all donors and acceptors are ionised one obtains:

$$
V_{\mathrm{bi}}=\frac{k T}{e} \ln \left(\frac{N_{A} N_{D}}{n_{i}^{2}}\right)
$$


for the built in voltage and the width of the depleted region is calculated to be

$$
D=\sqrt{\frac{2 \epsilon \epsilon_{0}}{e}\left(\frac{1}{N_{A}}+\frac{1}{N_{D}}\right) V_{\mathrm{bi}}},
$$

where $e$ is the magnitude of the electron charge, $\epsilon_{0}$ is the permittivity in vacuum and $\epsilon$ is the relative permittivity of the semiconductor.

An external voltage is often applied in order to change the behaviour of the p-n junction. The external voltage $V$ is defined to be positive in the following discussion if it is applied as a forward bias and negative if it is applied as a reverse bias. A forward bias leads to the fact that the potential difference $\Delta V=\left(V_{\mathrm{bi}}-V\right)$ that is illustrated in Figure 2.4 decreases, while a reverse bias voltage leads to an increase in the difference. Applying a forward bias also increases the current that flows through the junction, while nearly no current flows for a reverse bias. With increasing reverse voltage the current stays constant at almost zero until the breakdown voltage is reached, at which point the current increases rapidly. An illustration of the current voltage characteristics of a p-n junction is shown Figure 2.5.

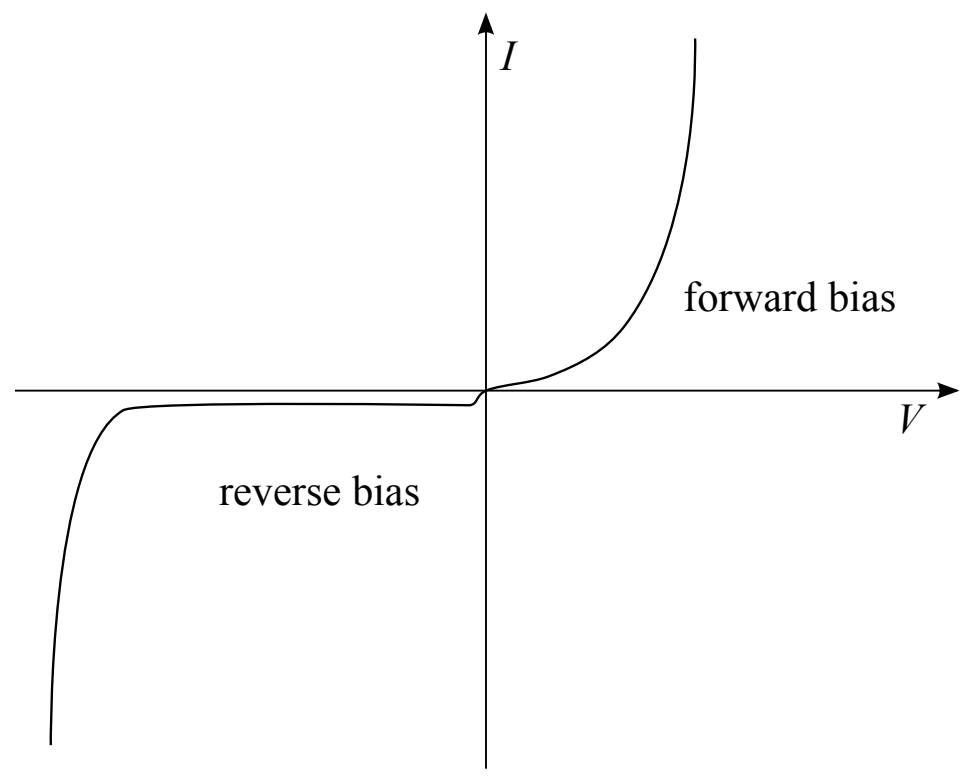

Figure 2.5.: Illustration of the current voltage characteristics of a p-n junction for forward and reverse bias voltages.

Furthermore, since the depleted region depends on the applied voltage it also varies in size if the bias voltage is changed:

$$
D=\sqrt{\frac{2 \epsilon \epsilon_{0}}{e}\left(\frac{1}{N_{A}}+\frac{1}{N_{D}}\right)\left(V_{\mathrm{bi}}-V\right)} .
$$

In general, semiconductor detectors are operated with a reverse bias voltage and only a small dark current (leakage current) is measurable. A charged particle passing through the sensor produces free charge carriers by ionisation, as mentioned previously. The created electron and hole pairs recombine in general quickly if they are created in the neutral region of the sensor, while they are separated in the depleted region due to the presence of the electric field. In order 
to increase the active region of the sensor and collect the created charge carriers it is thus desired to deplete the sensors fully.

In detectors, a highly asymmetric p-n junction is often used as the basic sensor design. A thin and highly doped p-implant $\left(N_{A}>10^{18} \mathrm{~cm}^{-3}\right)$ is combined with a larger bulk that is only doped with a low concentration of donors $\left(N_{D} \approx 10^{12} \mathrm{~cm}^{-3}\right)$ [22]. The size of the depleted region is mainly defined by its depth in the bulk since the implant is very thin. Thus, the term $\frac{1}{N_{A}}$ in Equation 2.21 can be neglected. In addition, the built-in voltage is in general small compared to the applied reverse bias and can therefore also be neglected. Hence, Equation 2.21 can be simplified to:

$$
D \approx \sqrt{\frac{2 \epsilon \epsilon_{0}}{e N_{D}}(-V)},
$$

and for the formula of the voltage that is needed to deplete the diode fully thus follows directly [22]:

$$
\left|V_{\mathrm{depl}}\right| \approx \frac{e N_{D} S^{2}}{2 \epsilon \epsilon_{0}},
$$

where $S$ is the total thickness of the sensor. Equation 2.23 is only valid for unstructured diodes and the depletion voltage of semiconductor detectors used in high energy physics differs from the theoretical value. However, this approximation is a good orientation for the estimation of such a crucial sensor property.

\subsubsection{Shockley-Ramo Theorem}

Moving charge carriers produced by a passing particle that drift in an electric field induce electric charge on the electrodes of the detector. The collected charge in the readout electronics is then converted into an output signal. The Shockley-Ramo theorem 24] offers a simple way to calculate the charge that is induced, $Q$, in an electrode by a free moving point charge $q$. For the calculation only one electrode $L$ of the sensor is selected. A charge $q$ moving from point $\vec{x}_{i}$ to $\vec{x}_{f}$ induces the following charge on $L[24]$ :

$$
Q_{L}=\int_{\vec{x}_{i}}^{\vec{x}_{f}} q \vec{E}_{0} \cdot \mathrm{d} \vec{x}=-q\left[\varphi_{0}\left(\vec{x}_{f}\right)-\varphi_{0}\left(\vec{x}_{i}\right)\right],
$$

where $\vec{E}_{0}$ is the weighting field and $\varphi_{0}$ is the weighting potential. The weighting field and potential can be estimated by selecting an electrode, which is set to the dimensionless unit potential 1, while all other electrodes are at ground potential and all charges are removed. It can then be calculated easily by solving the Poisson equation for the given configuration as long as the layout of the electrodes in the detector is known. The weighting potential can thus take values between 0 and 1 and it describes the induced charge on the electrode as a fraction of the free moving charge.

\subsubsection{Sensors for Pixel Detectors}

The ATLAS Pixel detector uses the so called hybrid technology for its sensors. This means that the sensor and the readout electronics are manufactured separately and joined afterwards. This section focuses on the general design of n-in-n sensors that are often used in high energy physics applications, while details about the ATLAS pixel detector including the electronics are discussed in Chapter 4. 


\section{Design}

The general design of an n-in-n sensor is based on the asymmetric p-n junction that has been discussed earlier. An illustration of the layout is given in Figure 2.6. N-in-n sensors are often used if radiation hardness is crucial, which is the case in the ATLAS pixel detector. The back side of the shown sensor is highly p-doped, while the n-bulk is only lightly doped. Highly ndoped pixels are added at the top of the sensor in order to achieve a high granularity of the detector. Each pixel is connected to the readout electronics, while the backside is not used for charge collection.

Sensors are usually cut at the side during their manufacturing, which leads to the creation of many crystal defects close to the edge of the sensor. These defects induce a very high current across the sensor, if the region at the edge is depleted. In order to avoid these effects, the electric potential in the detector is reduced in a controlled way by several guard rings which are placed at the edge of the sensor.

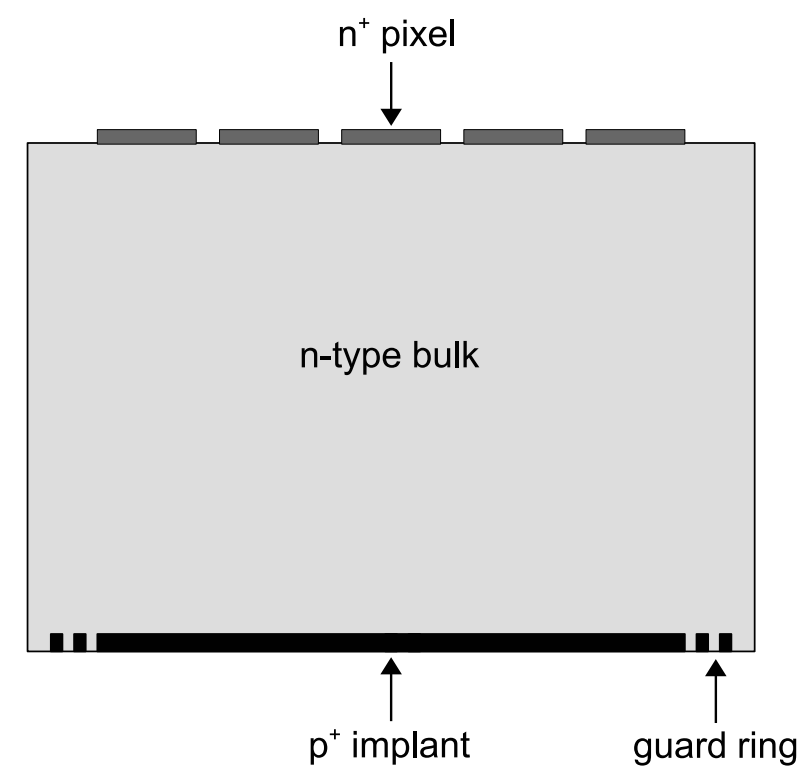

Figure 2.6.: Schematic view of an n-in-n pixel sensor.

\section{Charge Collection}

A high voltage is applied in order to operate the sensor fully depleted under normal conditions. Electrons are accelerated towards the pixel side, while holes drift towards the back side of the sensor, as mentioned previously. According to the Shockley-Ramo theorem, the induced signal depends on the weighting potential of the sensor. In detectors the size of a pixel is often in the same order of magnitude as the wafer thickness - this layout has a large influence on the induced charge. A simulation of the weighting potential for a pixel detector is shown Figure 2.7. The weighting potential was calculated for the pixel that was hit by a particle passing through the sensor perpendicularly and its neighbours indicated by the numbers.

A particle passing through the sensor creates electron hole pairs all along its path and an electron hole pair that is created in the centre of the sensor is taken as an example for the following discussion. In a non pixelated pad detector the weighting field is linear and it is assumed that both charge carriers travel all the way to the end of the sensor along the electric 

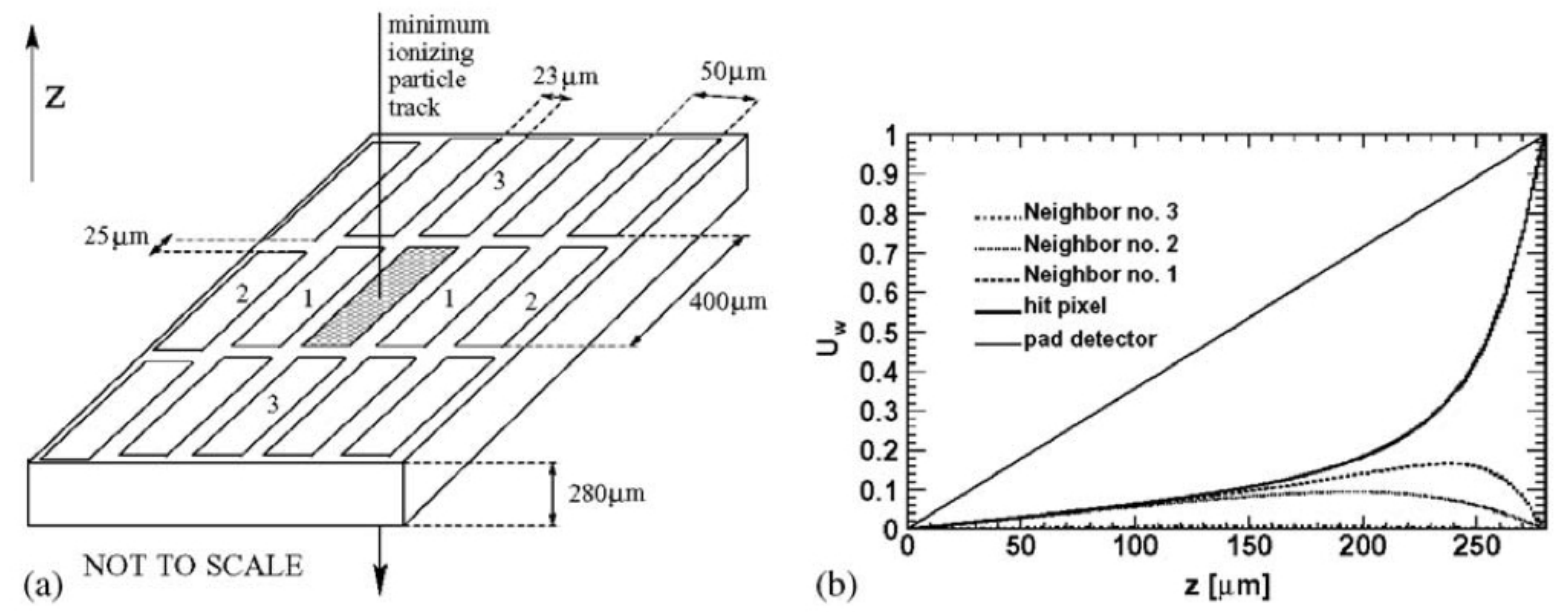

Figure 2.7.: Simulation of a pixel segment of an ATLAS pixel detector module with a sensor thickness of $280 \mu \mathrm{m}$ and a pixel size of $40 \times 250 \mu \mathrm{m}^{2}$ (a). The weighting potential is calculated for the grey pixel and its neighbours. The calculated weighting potential as a function of the sensor depth is given in (b). The hit pixel and its neighbours are shown and the weighting potential for a pad detector is added as a comparison 25 .

field lines. Using Equation 2.24 one obtains that the electron will induce a charge fraction of $\frac{e}{2}$ in the electrode while the hole will also contribute $\frac{e}{2}$ leading to a total induced charge of $e$. On average one half of the induced charge in pad detectors is thus created by the hole current and the other half by the electron current.

In pixel detectors the signal is mainly created by electrons. Due to the configuration of the electrodes the weighting potential is much larger at the pixel side than it is at the backside of the sensor. Therefore, electrons created at the back side of the sensor need to travel towards the pixels before they induce a large fraction of the signal, while holes travel towards the back side of the sensor and thus only induce a large fraction of the signal if they are created close to the pixel side.

Particles travelling through the sensor generally induce charge into a cluster of adjacent pixels, which is then used to extract precise information on the particle position. However, as mentioned earlier it is common to operate pixel detectors in the presence of a magnetic field. Thus the Lorentz angle has a large impact on the formation of clusters in a pixel detector. The effect is shown in Figure 2.8. The Lorentz effect is only illustrated for electrons, since they are the dominant factor for charge collection in pixel detectors and the concept is similar for holes. If the magnetic field is turned off, the cluster size is minimal for a track passing the sensor perpendicularly. In the presence of a magnetic field the cluster size increases as it is shown in Figure 2.8(a). The solid line represents a highly energetic charged particle passing through the sensor, while free electrons that drift towards the pixel implants are illustrated by the dashed lines. Pixels in which charge is induced are shown as white rectangles, while no signal is seen in the black pixels. The minimum cluster size is shifted in the presence of a magnetic field, as it is illustrated in Figure 2.8(b). This change in the expected cluster size is often so large that it needs to be taken into account while designing the detector and during the reconstruction of particle tracks in order to achieve full detector performance. 


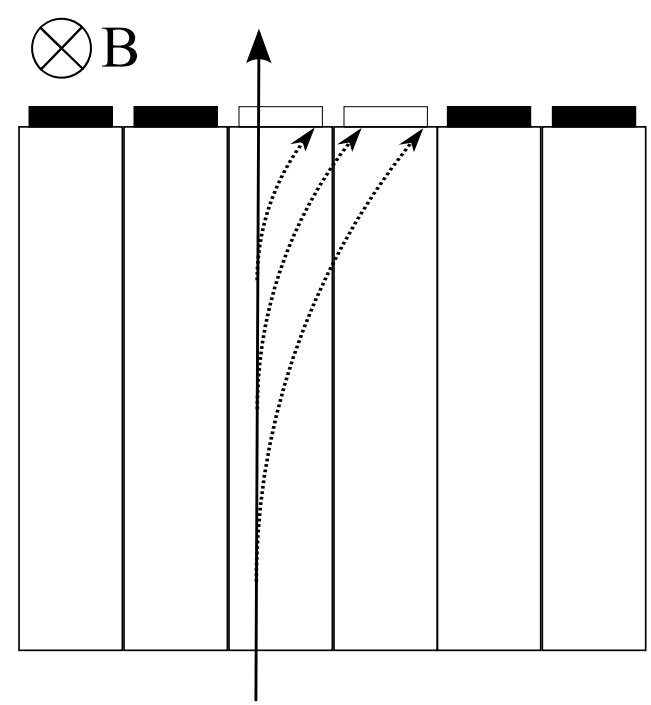

(a)

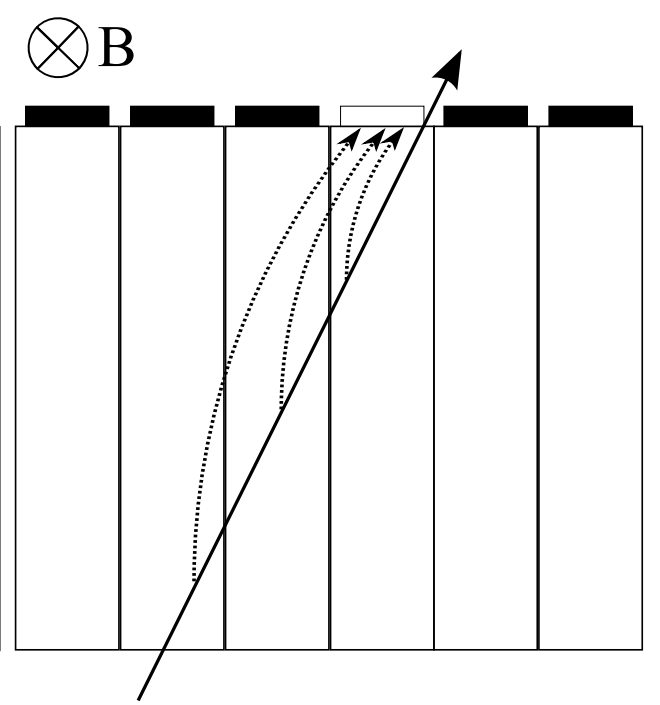

(b)

Figure 2.8.: Charge collection in the pixel sensor with magnetic field turned on. A particle crossing the sensor perpendicularly (a) and a particle crossing the sensor at the Lorentz angle (b) is shown. 



\section{The LHC and the ATLAS Detector}

\subsection{Introduction}

The Large Hadron Collider (LHC) at CERN ${ }^{1}$ is currently the most powerful particle collider in the world. As mentioned, goals of the LHC are the discovery of new particles and new physics beyond the Standard Model. For this purpose, several detectors are located in the accelerator ring. The four largest experiments at the LHC are ALICE [26], ATLAS [27], CMS [28], and LHCb [29]. ATLAS is one of the two general purpose experiments of the LHC. An introduction of the accelerator complex at CERN is given in Section 3.2, while the ATLAS detector is described in Section 3.3 .

\subsection{Large Hadron Collider}

The LHC is mainly designed for proton-proton collisions with a maximum centre-of-mass energy of $14 \mathrm{TeV}$ [30], while it is also possible to collide heavy ions $(\mathrm{Pb})$. Interesting physics events that are studied at the LHC are created very rarely, thus it is important to have a very high event rate $R$ in the LHC. The number of produced events per second is described as $R_{\text {event }}=L \sigma_{\text {event }}$, where $L$ is the luminosity of the accelerator and $\sigma_{\text {event }}$ is the cross section of the corresponding physics event. Therefore, it is desirable to achieve a high luminosity in the LHC. The luminosity $L$ is a function of the beam parameters 30 :

$$
L=\frac{N_{b}^{2} n_{b} f_{\mathrm{rev}} \gamma_{r}}{4 \pi \epsilon_{n} \beta *} F
$$

where $f_{\text {rev }}$ is the revolution frequency, $\gamma_{r}$ the relativistic gamma factor, $\epsilon_{n}$ the normalized transverse beam emittance, and $\beta *$ the beta function at the collision point, which is related to the transverse size of the particle beam. $F$ is the geometric luminosity reduction factor. The reduction factor takes into account the angle at which the bunches cross at the interaction point. The beam in the LHC is segmented into several packages of protons. Each one of them is called a bunch and $n_{b}$ is the number of bunches per beam, while $N_{b}$ is the number of protons per bunch. The LHC is designed to deliver a peak luminosity of $10^{34} \mathrm{~cm}^{-2} \mathrm{~s}^{-1}$ for protons in ATLAS and CMS.

The accelerator ring of the LHC consists of two beam pipes and superconducting magnets. The LHC is placed in the old $26.7 \mathrm{~km}$ long tunnel. It was constructed for the Large ElectronPositron Collider (LEP) that was built between 1984 and 1989. The LHC consists of eight arcs and eight straight sections. The straight sections are about $528 \mathrm{~m}$ long and they house the experiments and the machinery for beam injection.

The particle beam is bent by 1232 superconducting dipole magnets. The counter rotating proton-proton beams require opposite magnetic dipole fields. Thus, the magnets have been designed to hold two beam pipes with two isolated magnetic fields. Each magnet is approximately

\footnotetext{
${ }^{1}$ Conseil Européen pour la Recherche Nucléaire
} 
$15 \mathrm{~m}$ long and it is cooled to a temperature below $2 \mathrm{~K}$. Thus, it is possible to operate the magnets with a magnetic field of about $8 \mathrm{~T}$ at a beam energy of $7 \mathrm{TeV}$.

The LHC injection chain for protons consists of the Linac2, the Proton Synchroton Booster (PSB), Proton Synchroton (PS) and the Super Proton Synchrotron (SPS). A schematic view of the CERN accelerator complex is shown in Figure 3.1 .

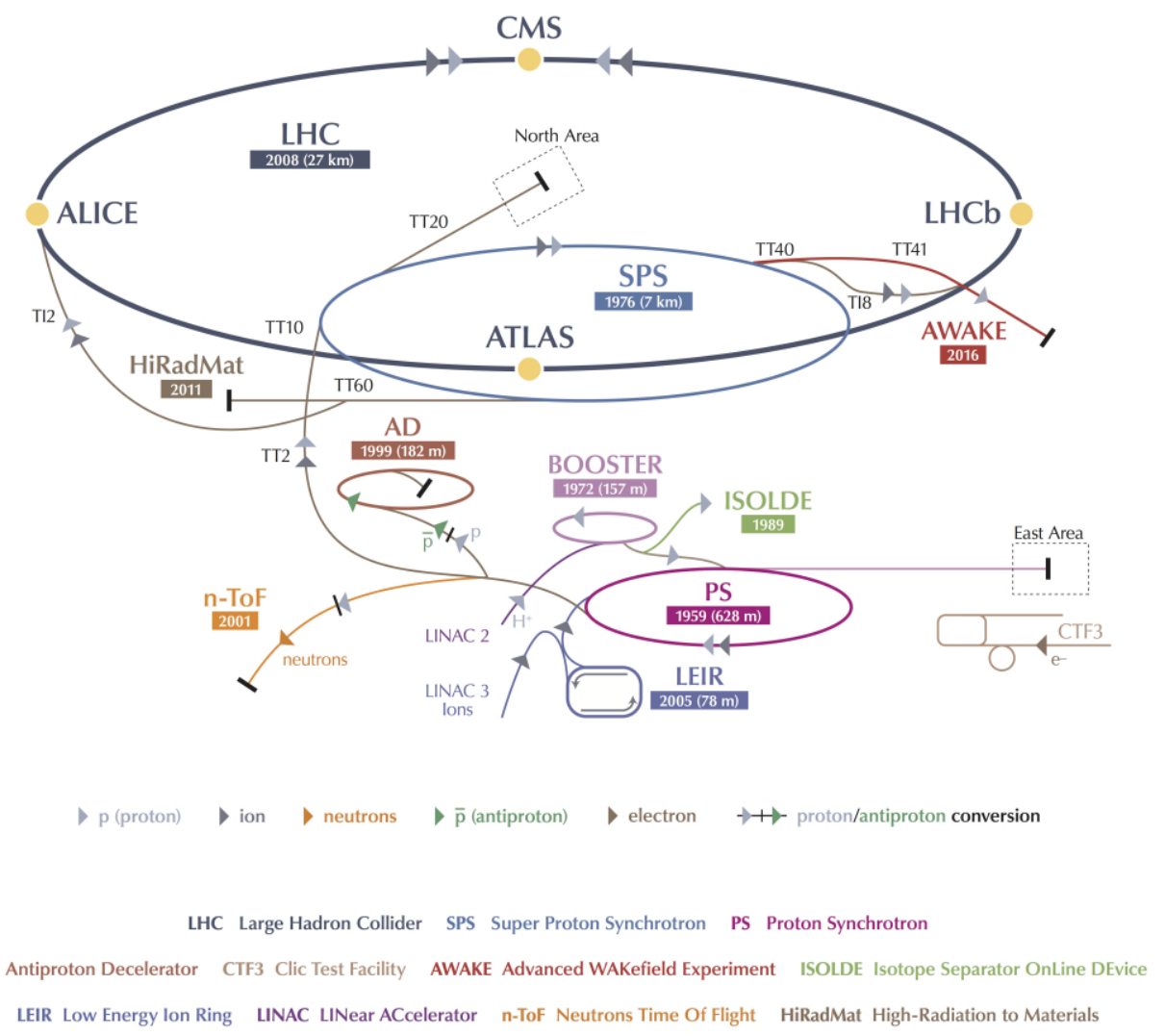

Figure 3.1.: Overview of the LHC accelerator complex [31]. Protons are injected by Linac2 and passed through the Booster, PS and SPS before they are transferred to the LHC.

Protons are injected into Linac2 and accelerated to an energy of $50 \mathrm{MeV}$, after which they are transferred to the PSB. In the PSB, particles are accelerated to an energy of $25 \mathrm{GeV}$ and transmitted to the SPS. The SPS increases the beam energy further up to $450 \mathrm{GeV}$. Two injection lines exist between the SPS and the LHC, one for each beam pipe of the LHC. Protons are injected-counter rotating in the two lines and they can be accelerated in the LHC up to a maximum beam energy of $7 \mathrm{TeV}$.

\subsection{ATLAS Detector}

The ATLAS detector is cylindrical with a length of $44 \mathrm{~m}$ and a height of $25 \mathrm{~m}$. The total weight is approximately $7000 \mathrm{t}$ [27]. The detector has a forward-backward symmetry and it covers the full angle around the interaction point. The detector consists of several subdetectors which are arranged in an onion like shape. An overview of the ATLAS detector and its layers is given in Figure 3.2 . 


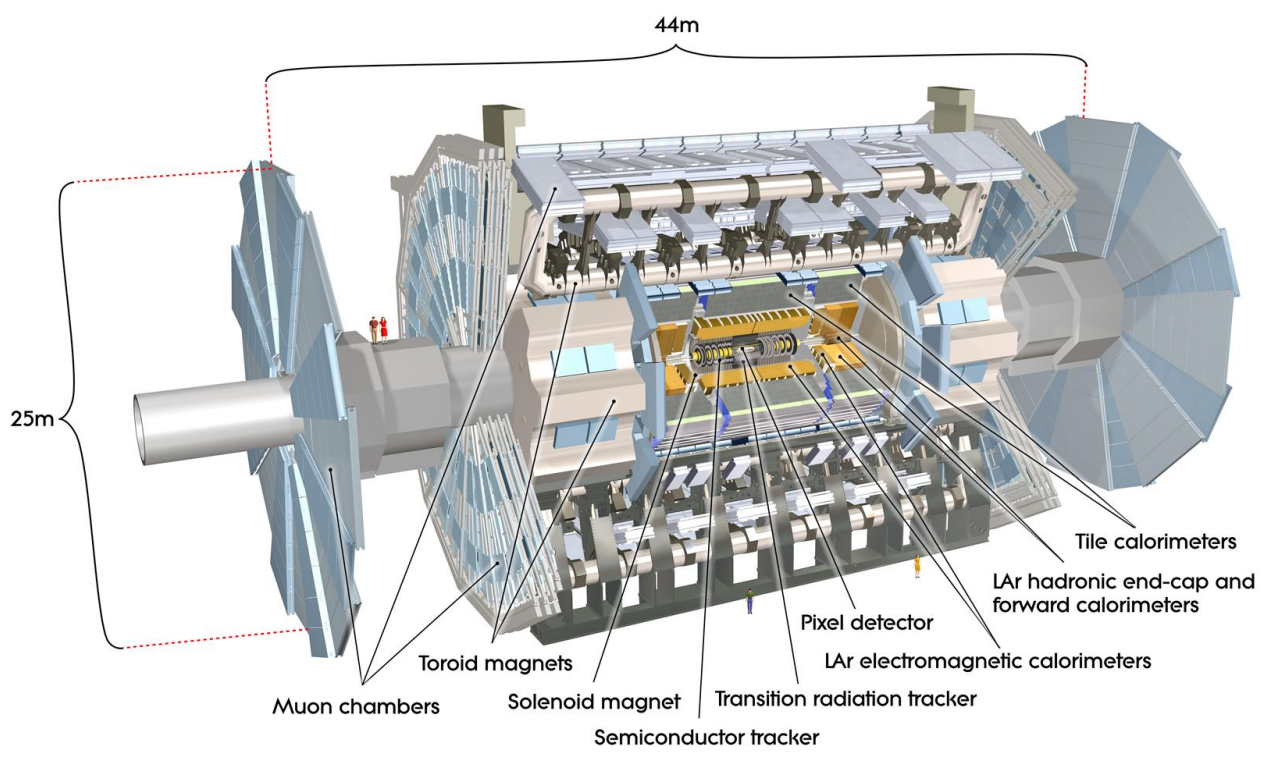

Figure 3.2.: Overview of the ATLAS detector and its subsystems 27].

The inner detector is responsible for vertex and momentum measurements. It is surrounded by a solenoidal magnet that produces a magnetic field of $2 \mathrm{~T}$. The calorimetry systems consist of a high granularity electromagnetic liquid-argon calorimeter and a hadronic scintillator-tile calorimeter. Eight toroidal magnet coils and two end-cap magnets are located behind the calorimeter within the muon system. They provide a large bending power, which leads to a high muon momentum resolution in the the muon chambers.

\subsubsection{Coordinate System}

Subsystems of the ATLAS detector as well as particle properties are described within a global coordinate system that has its origin at the interaction point. The z-axis is parallel to the beam line and it is inclined with respect to the horizontal, due to the fact that the beamline is inclined by $1.23 \%$. Positive $\mathrm{z}$ points slightly downhill towards LHCb. The positive-z side of the ATLAS detector is called A-side, while the negative side is called C-side. The $\mathrm{x}$-axis is horizontal and points towards the centre of the LHC accelerator ring and the y-axis points upwards and it is also inclined by $1.23 \%$ with respect to the vertical 32 .

Due to the symmetry of the ATLAS detector, a global spherical coordinate system is defined. The $R \phi$-plane of the detector is equal to the global xy-plane. $R$ is the radius and $\phi$ is the azimuthal angle measured relative to the x-axis, while $\Theta$ is the angle between the particle track and the beam line and the pseudorapidity is described as:

$$
\eta=-\ln \left(\tan \left(\frac{\Theta}{2}\right)\right) .
$$

\subsubsection{Inner Detector}

The inner detector is the high resolution tracking system of the ATLAS detector. It consists of three sub-detectors, the innermost detector being the pixel detector is followed by a strip 
detector, the Semi-Conductor Tracker (SCT). They cover a pseudorapidity range of $|\eta|<2.5$ [27. The outer part of the inner detector is covered by the Transition Radiation Tracker (TRT), which uses transition radiation for electron identification within a range of $|\eta|<2.0$. The system is illustrated in Figure 3.3 .

The magnetic field in the inner detector created by the solenoid bends the tracks of charged particles and thus offers the opportunity to measure the momentum of this particle.

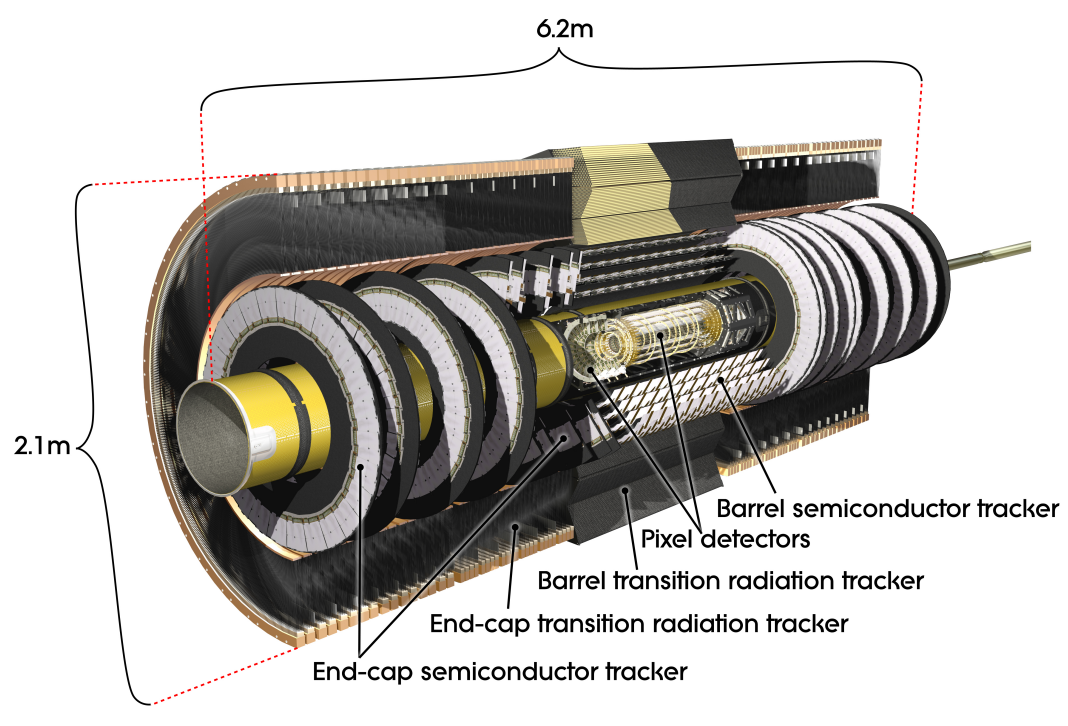

Figure 3.3.: ATLAS inner detector, with its three subcomponents, the pixel detector, the SCT and the TRT 27].

A detailed description of the ATLAS pixel detector is given in Chapter 4 , since the analysis that is presented in this thesis is closely related to the pixel detector.

The SCT uses strip detector technology instead of pixels, because strip modules were cheaper to produce at the time of the detector construction. SCT sensors use p-in-n technology with a strip pitch of $\sim 80 \mu \mathrm{m}$. In total the SCT consists of 4088 modules arranged in four barrel layers and 9 end-cap disks. Overall the SCT has a total silicon surface area of $63 \mathrm{~m}^{2}$. The four barrel layers are located at radii of $29.9 \mathrm{~cm}, 37.1 \mathrm{~cm}, 44.3 \mathrm{~cm}$, and $51.4 \mathrm{~cm}$.

The TRT combines particle tracking and identification. Relativistic charged particles passing through the boundary between two materials with varying dielectric constants emit transition radiation. The intensity of the transition radiation is a function of the lorentz factor $\gamma$. Heavier particles thus radiate less than lighter particles for a fixed energy. This dependence offers the opportunity to identify charged particles in the detector. The TRT consists of drift tubes with a diameter of $4 \mathrm{~mm}$, combined into 73 layers in the barrel and 160 straw planes in the end-caps. In the barrel, polypropylene fibres are interlaced in order to create transition radiation, while polypropylene radiator foils are used in the end-caps.

\subsubsection{Calorimetry}

The calorimeter system in the ATLAS detector covers a pseudorapidity range of $|\eta|<4.9[27$. A schematic view of the ATLAS calorimeter is given in Figure 3.4. The system consists of the electromagnetic and the hadronic calorimeter. Both systems use different technologies due to the changing physics requirements. The whole system is placed in three separate cryostats. The 
barrel cryostat houses the electromagnetic barrel calorimeter and the hadron barrel. The two end-cap cryostats hold the tile extended barrel, the electromagnetic end-cap calorimeters and the forward calorimeters.

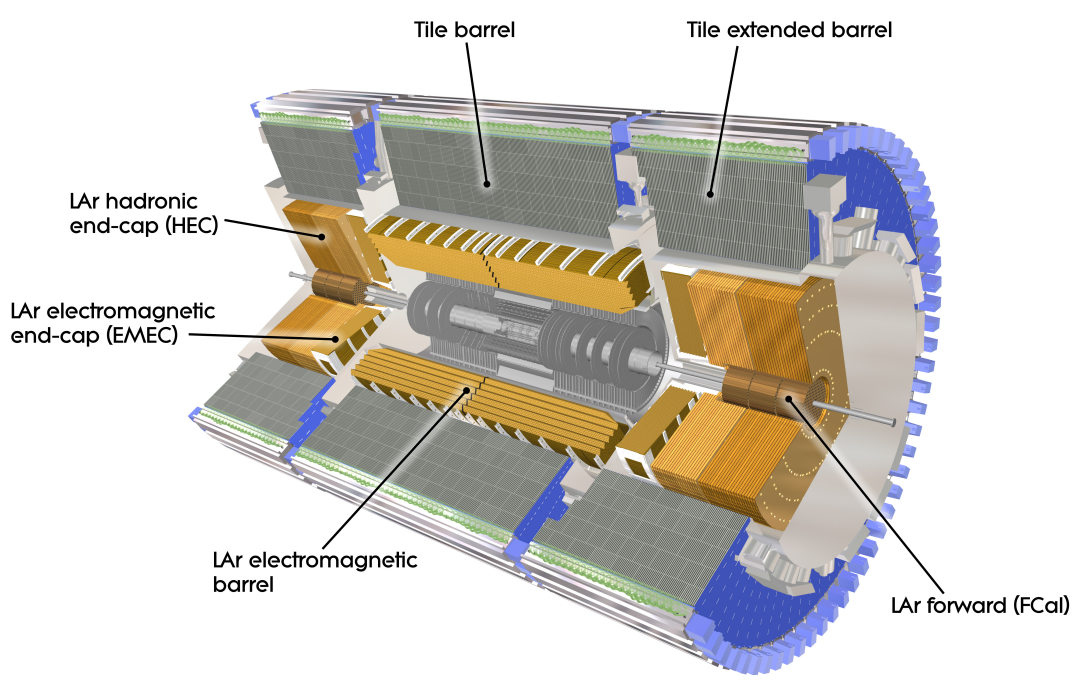

Figure 3.4.: Schematic view of the ATLAS calorimeter system [27].

The barrel part of the electromagnetic calorimeter is located in a range of $|\eta|<1.475$ and the two end-cap parts cover a range of $1.375<|\eta|<3.2$. Lead-liquid argon technology is used in the precise electromagnetic calorimeters. Their shape is accordion like and the gaps between the the lead absorbers are filled with liquid argon as the active medium. Liquid argon was chosen because it is intrinsically radiation hard.

The barrel is composed of two half barrels each with a length of $3.2 \mathrm{~m}$ and a weight of $57 \mathrm{t}$. The inner and outer diameters of the barrel are $2.8 \mathrm{~m}$ and $4 \mathrm{~m}$. The end-caps are composed of two wheels at the end of the barrel. The radiation length $\left(X_{0}\right)$ of the electromagnetic calorimeter is $>22$ in the barrel and $>24$ in the end-caps.

The hadronic calorimeter consists of a centre barrel, two extended barrels, the end-caps and a forward calorimeter. The tile calorimeters that are placed in the barrel region consist of steel absorbers and scintillating tiles in the active region. The signal is read out by photomultipliers that are connected via fibres. The barrel covers a region of $|\eta|<1.0$ and the extended barrels cover $0.8<|\eta|<1.7$. It has an inner radius of $2.28 \mathrm{~m}$ and an outer radius of $4.25 \mathrm{~m}$.

Hadronic end-caps are placed directly behind the electromagnetic end-caps. The end-cap wheels use the liquid-argon technology with copper plates as absorber material and a coverage of $1.5<|\eta|<3.2$. The liquid argon forward calorimeter covers a range of $3.1<|\eta|<4.9$ and is located behind the electromagnetic calorimeter end-cap.

\subsubsection{Muon System}

An overview of the muon system is given in Figure 3.5. In total four different types of chambers are used in the muon spectrometer [27]. Monitored Drift Tubes (MDT's) are located in a large pseudorapidity range allowing precise position measurements. In the innermost layer and at large pseudorapidities Cathode Strip Chambers (CSC's) are used, because they provide a higher counting rate. An important feature of the muon system is the ability to trigger on muons in 
a range of $|\eta|<2.7$. The trigger system consists of Resistive Plate Chambers (RPC's) in the barrel region and Thin Gap Chambers (TGC's) in the end-caps.

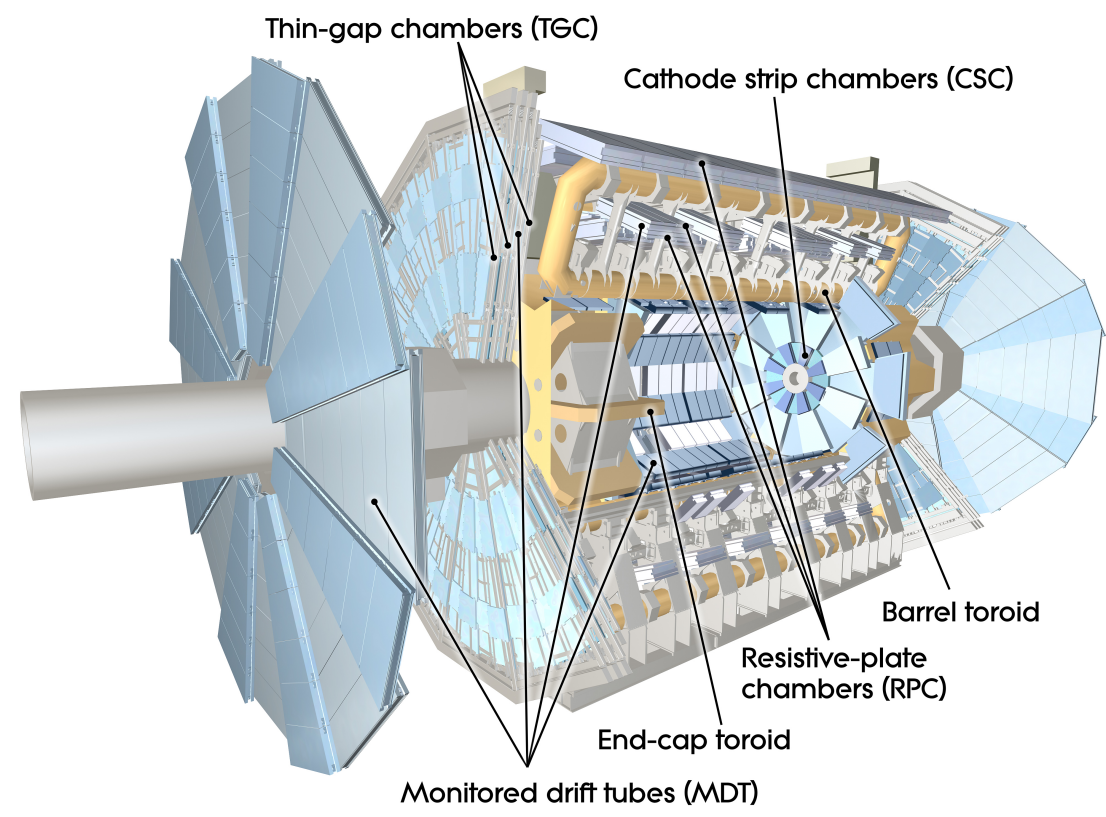

Figure 3.5.: Schematic view of the ATLAS muon system 27].

The muon chambers are placed within a toroidal magnetic field. In a range of $|\eta|<1.4$ the field is created by toroidal magnet coils and between $1.4<|\eta|<1.6$ it is provided by barrel end-cap magnets. The magnet system consists of one large air-core toroid in the barrel and two end-cap toroids. The air-core toroid is composed of eight coils, while the end-cap toroids hold eight racetrack-like coils which are housed in an aluminium alloy enclosure.

The bending power of a magnet is defined by $\int \mathrm{B} \cdot \mathrm{dl}$. B is the component of the magnetic field that is normal to the direction of the passing muon. The integral is calculated between the innermost and outermost muon chamber for a muon with infinite momentum. A bending power of 1.5 to $5.5 \mathrm{Tm}$ is provided by the toroid in a range of $0<|\eta|<1.4$, while the end-cap magnets deliver about 1 to $7.5 \mathrm{Tm}$ in a range of $1.6<|\eta|<2.7$. The bending power is not as large in the region in-between the two areas.

MDT's cover a range of $|\eta|<2.7$. In the innermost layer the MDT's are replaced by the CSC's in a range of $2<|\eta|<2.7$, as mentioned above. 1088 MDT chambers have been installed covering an area of $5500 \mathrm{~m}^{2}$. The MDT chambers consist of drift tubes that are operated with $\mathrm{Ar} / \mathrm{CO}_{2}$ gas. The tubes have a diameter of $29.970 \mathrm{~mm}$ and hold a tungsten-rhenium wire with a diameter of $50 \mu \mathrm{m}$ in the centre.

The counting rate in the high $\eta$ region exceeds the maximal rate of the MDT's $\left(\sim 150 \frac{\mathrm{Hz}}{\mathrm{cm}^{2}}\right)$, while CSC's can be operated up to a rate of about $1000 \frac{\mathrm{Hz}}{\mathrm{cm}^{2}}$. CSC's are multiwire proportional chambers and they cover a total area of approximately $65 \mathrm{~m}^{2}$. The cathodes of the CSC's are segmented into strips, one parallel and one perpendicular to the wires. The position of the passing muon can be obtained by interpolating the two signals coming from the strips. The distance between the anode wires $d$ is equal to the distance between the wires and the cathode strips $S=d=2.5 \mathrm{~mm}$. The induced signal in the wires is not used, since it is sufficient to use the signal of the strips.

RPC's are used in the barrel region of the trigger system and they do not possess any wires 
which leads to a simpler design. They have a good time resolution and a sufficient counting rate. The requirements for trigger chambers in the end-caps are more demanding, thus the TGCs that are used in the end-caps are also based on multi-wire proportional chambers. They have been developed to deliver a good time resolution and function at high counting rate.

\subsubsection{ATLAS Trigger System}

The trigger system of the ATLAS detector is composed of the Level-1 (L1) trigger and the High Level Trigger (HLT) 27]. The L1 trigger system uses only a part of the ATLAS subdetectors to make a selection. In the muon system only the RPC's and TGC's are taken into account, while the whole calorimeter is used. The L1 trigger level reduces the readout rate from $40 \mathrm{MHz}$ to about $75 \mathrm{kHz}$. Interesting signatures are saved and passed to the next system.

The Level-2 (L2) trigger and the event filter together form the High Level Trigger. Only detector information of the selected event in a region of interest is used in the L2 trigger level in order to improve the selection of events. The region of interest corresponds to the region in the detector where a possible object was found by the L1 trigger in the selected event.

Candidates passed to the event filter are assessed with information from all subdetectors and offline analysis techniques in order to refine the selection. The readout rate is reduced to about $200 \mathrm{~Hz}$. Events selected by the event filter are considered important enough to be recorded permanently. 



\section{The ATLAS Pixel Detector}

\subsection{Introduction}

As the innermost detector layer, the ATLAS pixel detector plays a crucial role in track reconstruction. It is a very important detector for the identification of secondary vertices and thus it plays a vital role for the performance of b-tagging measurements. The detector has a very high spatial resolution, which enables a very precise reconstruction of primary vertices created by the proton-proton interactions. It is designed for the detection of particles in a pseudorapidity range of $|\eta|<2.5$. The high peak luminosity in the LHC required the installation of approximately 80 million channels for the pixel detector.

The pixel detector has the advantage of delivering real 2-dimensional information and is therefore not affected by ambiguity problems as strip detectors are. Furthermore, radiation hardness of the detector is crucial and the pixel detector has the advantage that effects induced by radiation damage only have a considerable impact at very high fluences. It is designed to withstand a total dose of $500 \mathrm{kGy}$ and a fluence of about $10^{15} \mathrm{n}_{\mathrm{eq}} \mathrm{cm}^{-2}$. It is expected that the lifetime-dose is reached after about 5 years of operation in the innermost layer, while the other layers are expected to reach the dose after 10 years or more 33 .

The small pixel size leads to a very high resolution of the detector. In addition, the innermost pixel layer is placed as close as possible to the interaction point allowing high precision tracking. The amount of material in the detector is kept as small as possible to deal with multiple scattering and photon conversions.

An overview of the detector is given in Section 4.2. The whole system consists of single pixel modules, which are introduced in Section 4.3 . The readout system of the ATLAS pixel detector consists of an electrical and optical part, a short summary of this system is given in Section 4.4 .

\subsection{Layout of the Pixel Detector}

The ATLAS pixel detector consists of three barrel layers and it is designed to cover a pseudorapidity region of $|\eta|<2.5$. Layer 0,1 , and 2 have radii of $50.5,88.5$, and $122.5 \mathrm{~mm}[33,34]$. The ends of the detector are covered by two end caps which are composed of three disk layers each.

In addition, the internal services (cooling, monitoring, power, optical input and output) and the corresponding mechanical support structures are located on both ends of the active region of the detector. A pixel support tube holds the active detector part, the services as well as related support structures. The external services are connected at the end of the pixel support tube to the internal services.

The innermost pixel layer (layer 0 or b-layer) is composed of 22 staves, while layer 1 consists of 38 staves and layer 2 of 52 . A schematic view of the pixel detector is given in Figure 4.1. A stave holds 13 pixel modules and the cooling pipes of the detector. The disks consist of 48 modules, resulting in a total of 1744 pixel modules with a sensitive area of about $1.7 \mathrm{~m}^{2}$. All staves are tilted by $20^{\circ}$ in order to optimize the cluster size distribution of the incoming particle tracks and to compensate the Lorentz-displacement of charges in the magnetic field. 


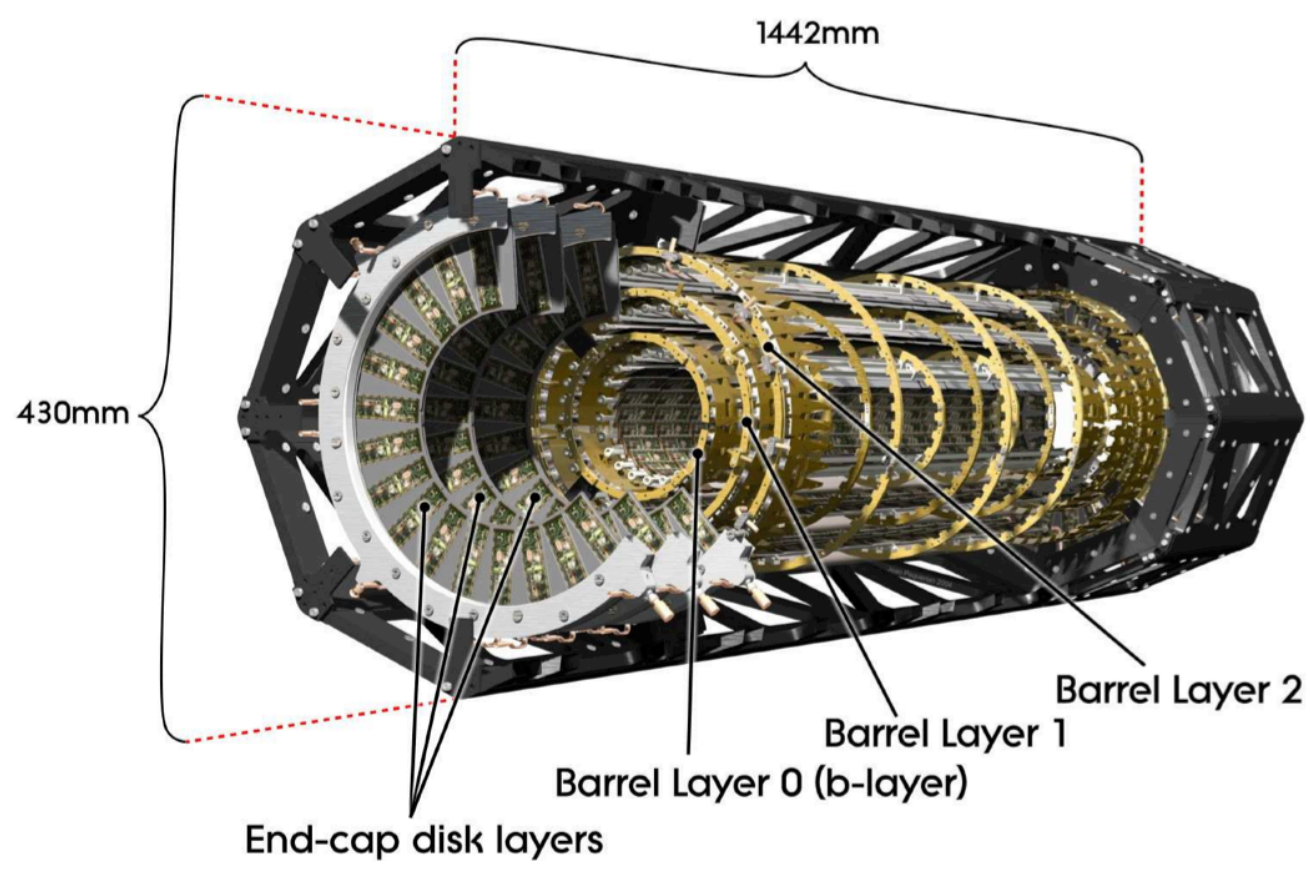

Figure 4.1.: Overview of the ATLAS pixel detector and its support structure 33. The detector is composed of three layers and two end-cap regions.

\subsection{Pixel Module}

The design of each pixel module in the detector is identical. A single pixel module has an active surface area of about $6.08 \times 1.64 \mathrm{~cm}^{2}$ and it is composed of three main components 33 :

- The flex-hybrid that holds the control chip, circuits for low and high voltage distribution as well as data connections.

- A silicon sensor with an n-in-n design, which is used as the active area for the collection of charge carriers.

- Sixteen Front-End chips (FE chips) which are responsible for the signal collection and digitization of the sensor output.

An illustration of a pixel module and its essential parts is given in Figure 4.2(a), The electrical connection between the FE-chip and the pixels on the sensor is realised via the so called bumpbonds. Bump-bonds are small metal spheres. The bump-bond connection is illustrated in Figure 4.2(b), The electrical connection between the FE chips and the flex-hybrid is realised with wire bonds. 


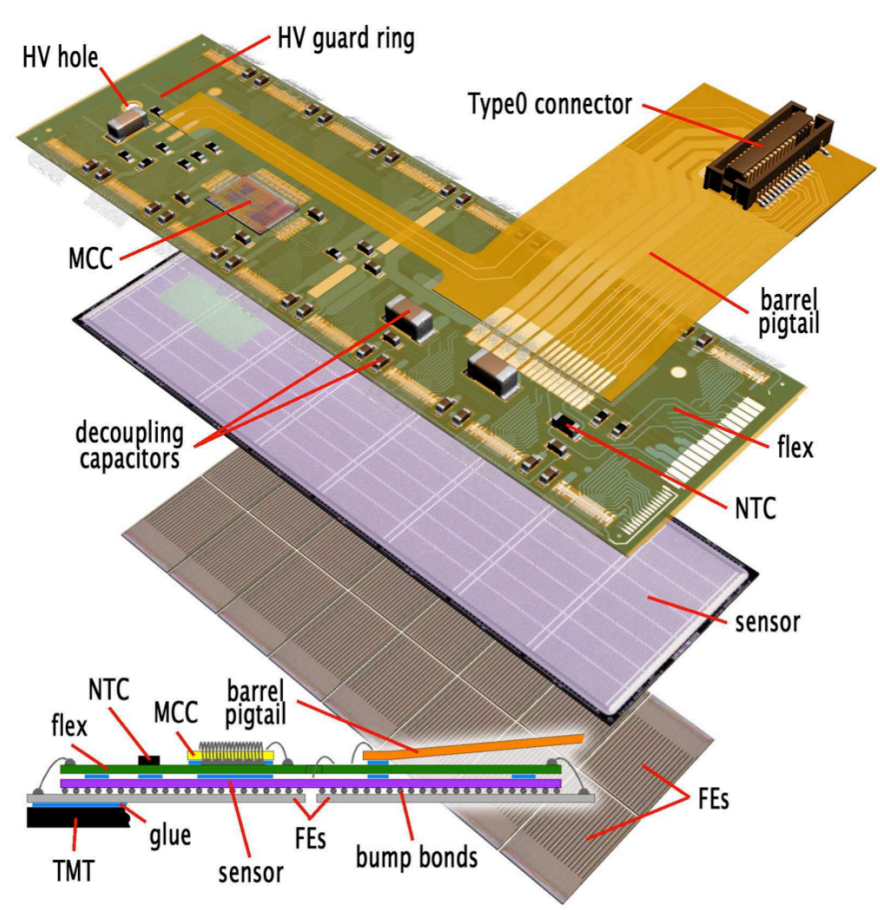

(a)

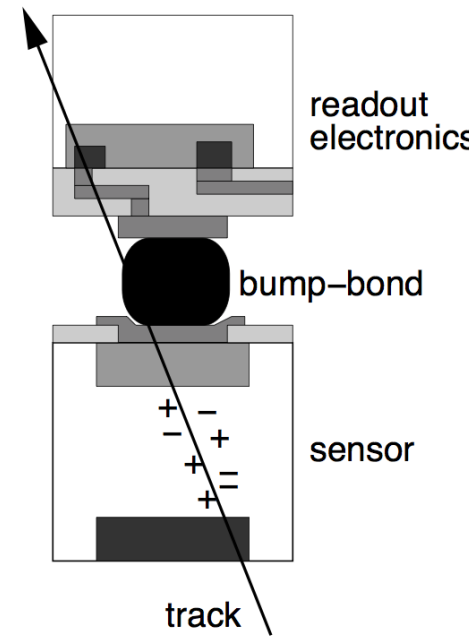

(b)

Figure 4.2.: Schematic drawing of the pixel module (a). A single module consists of the flexhybrid, the sensor and the FE-chips. The electric connection between the sensor and the FE chips is illustrated (b). Small metal spheres, the so called bump bonds, are used to establish the connection [33.

\subsubsection{Flex-Hybrid}

The flex-hybrid is a double-sided flexible printed circuit board with a thickness of approximately $100 \mu \mathrm{m}$ [33]. It holds the control chip, the Negative Temperature Coefficent (NTC), the electrical connections for high and low voltage power supplies, and data in and output lines. The NTC is used for the monitoring of the module temperature. An interlock system powers the module off quickly, if the temperature is too high.

The Module Control Chip (MCC) needs to operate in a high radiation environment with a time resolution of $25 \mathrm{~ns}$. The first radiation soft chips were produced in 1998. Several improvements led to the design of the final MCC-I2.1 chip in 2003 [35].

It has three main tasks. First, the MCC configures itself and the FE chips before a data-taking run or after power-up. Second, the MCC is responsible for the distribution of timing signals such as bunch crossing, L1 triggers and resets, and readout signals for the FE chips. Third, event building is done by the MCC. It is the most complicated task, data coming from the FE chips is buffered and used by the event builder to prepare the transmission of an event out of the pixel module.

External signals and the supply voltage are transmitted through the pigtail in barrel modules, while for the disk module wires are soldered directly to the flex, without the pigtail. 


\subsubsection{The Pixel Sensor}

The sensitive area of the pixel detector is a silicon sensor with high resistivity. It has a $256 \pm 3 \mu \mathrm{m}$ thick n-type bulk. The $\mathrm{n}^{+}$pixel implants are located on the readout side of the bulk. The backside of the sensor is p-doped in order to create a p-n junction. Each sensor consists of 47232 pixel implants [33].

The pixel size in the short pixel direction is $50 \mu \mathrm{m}$ and in the long pixel direction it is $400 \mu \mathrm{m}$. The gap between two FE chips is $400 \mu \mathrm{m}$ wide and thus needs to be covered by a special type of pixel. Figure 4.3 shows the pixel region at the edge of the FE chips. Elongated pixels with a length of $600 \mu \mathrm{m}$ cover the gaps in the long pixel direction, while ganged pixels are used to cover the gap in the short pixel direction. At the corner of each FE chip, elongated + ganged pixels are used in order to achieve full coverage.

Four ganged pixels are connected to four pixels at the edge of each FE chip. In order to minimize the impact on track reconstruction ganged pixels are always connected to the next but one neighbouring pixel below the FE chip. The signal between the bump-bonded and the ganged pixel hits can be distinguished, because a hit in two neighbouring ganged pixels is recorded as a hit in non-neighbouring bump bonded pixels.
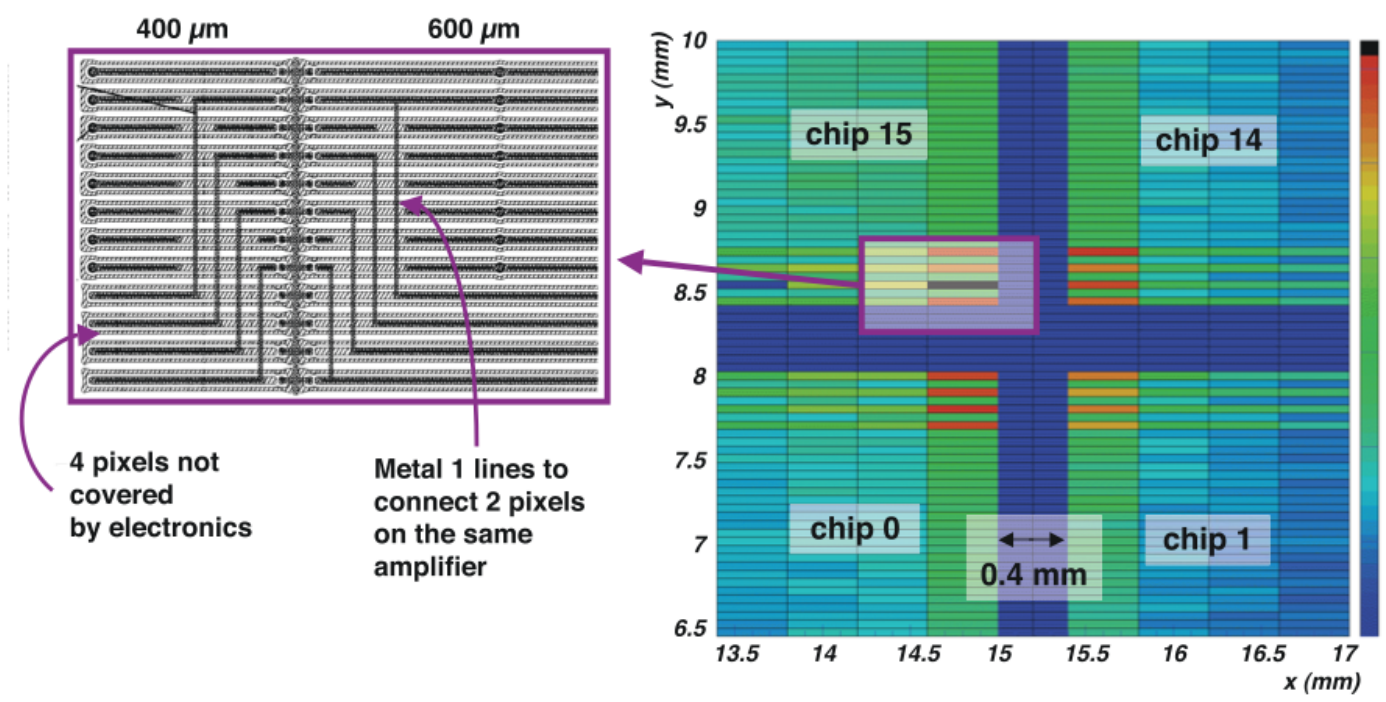

Figure 4.3.: Interchip region of the pixel detector. Long and ganged pixels are used to cover the gap between the FE chips [33].

The edges of the sensors are damaged by the cutting of the pixel sensors. This leads to crystal defects in the silicon close to the sensor edge. These defects can create shorts and increase the leakage current if this region is depleted. Thus, 17 guard rings are implemented to decrease the potential stepwise towards the cutting edge of the sensor [36].

The pixel sensor is operated with a reverse bias voltage in order to extend the depletion depth through the whole sensor bulk. Electron hole pairs are separated in the depleted region and can thus be collected and detected as it is explained in detail in Chapter 2, The charge carriers are deflected in the presence of a magnetic field due to the Lorentz force as discussed previously. The magnetic field in the ATLAS pixel detector is parallel to the beam line and thus perpendicular to the drift direction. Charge carriers are only deflected in the short pixel direction, due to the orientation of the magnetic field and of the pixel modules. Long pixels are parallel to the 
magnetic field and the effect on the charger carrier drift is negligible.

The Lorentz angle is an important effect that needs to be taken into account during the reconstruction of particle tracks. More detailed information and testbeam measurements of the Lorentz angle can be found in $33,37,38]$.

The depleted region starts to grow at the backside of the sensor before type-inversion. The sensor has been designed to be tolerant to radiation damage. The impact of radiation damage is explained and analysed in Chapter 6 .

\subsubsection{Front End Electronics}

Each module of the ATLAS pixel detector is read out by $16 \mathrm{FE}$ chips. The FE-I3 readout chip is implemented as a standard $0.25 \mu \mathrm{m}$ CMOS chip with a radiation tolerant layout 39,40 , 33. The chip consists of 2880 pixel cells, arranged in 18 columns and 160 rows. The cells are designed to digitize the signals coming from the corresponding pixel in the sensor.

An overview of the analogue readout part is shown in Figure 4.4. The sensor and the FE chips are connected via bump bonds. The unit cell contains two amplifiers and a discriminator with an adjustable threshold. A capacitor $\left(C_{f}=6.5 \mathrm{fF}\right)$ is charged by the induced current and it is discharged through a constant feedback current. The outcome is a nearly triangular signal shape, where the rising edge is defined by the collected charge in the sensor, while the falling edge is defined by the feedback current.

The amplitude of the feedback current is tuned globally for every FE chip with an 8-bit DAC 1 (IF DAC) and locally for every pixel with a 3-bit DAC (FDAC). A calibration circuit is included in each unit cell. The capacitors $C_{\text {low }}$ and $C_{\text {high }}$ are used to inject predefined amounts of charge into the pixel unit cell.

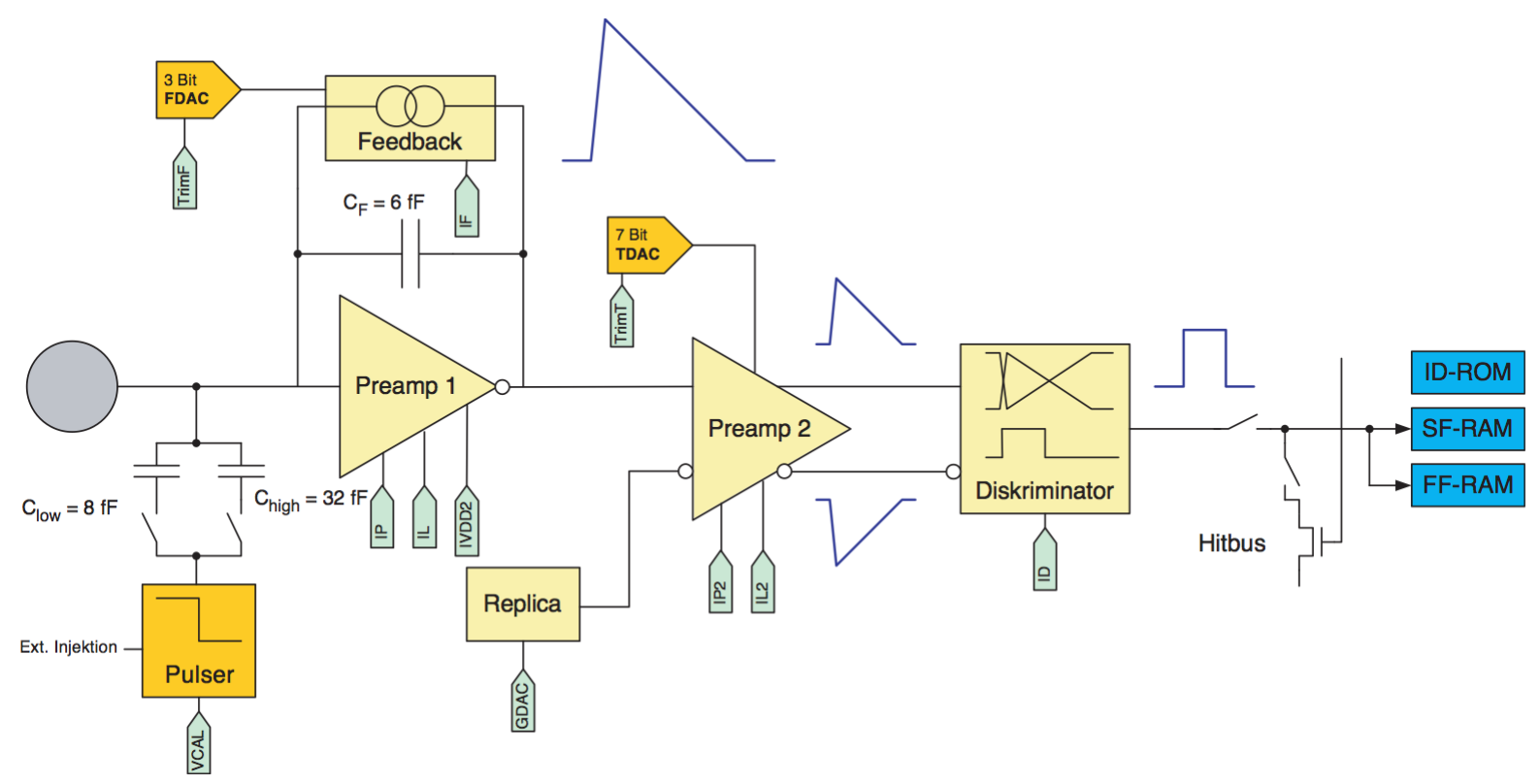

Figure 4.4.: Overview of the analog part of the pixel unit cell in the FE-I3 chip 39].

The discriminator output is a logical one as long as the incoming signal is above the adjustable threshold. An example of the signal digitization is given in Figure 4.5. The value that is saved

${ }^{1}$ Digital Analogue Converter 
is the Time over Threshold (ToT), i.e. the total time that the signal exceeds the discriminator threshold. Two cases are shown, a signal with a large amount of collected charge and a smaller one. The ToT value is proportional to the collected charge in the sensor and it is measured in units of $25 \mathrm{~ns}$. The threshold of the discriminator can be tuned globally with a 5-bit Global DAC (GDAC) and locally with a 7-bit Trim DAC (TDAC).

The hit coordinate as well as a timestamp for the leading edge of the discriminator output signal are temporarily stored in one of the 64 buffers per column pair until the readout selection is made by the trigger. Hits selected for readout are sent to the module controller chip (MCC) which builds events containing the hit information of all $16 \mathrm{FE}$ chips. Hits without a trigger signal are discarded.

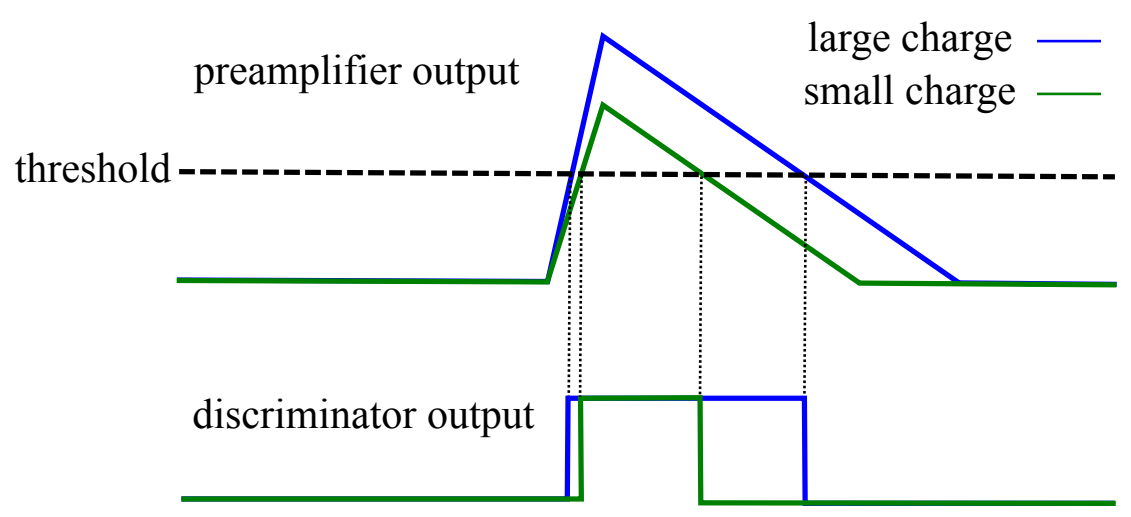

Figure 4.5.: Digitization of the sensor signal. The output of the first preamplifier and of the discriminator in the FE chip is illustrated. The dependence on the collected charge is shown.

\subsection{Readout System and Optoboards}

The communication between the pixel modules and the off-detector electronics is established with optical links 33,41 . The architecture is based on the ATLAS SCT implementation [42]. The design has been adapted to the requirements of the pixel detector (radiation hardness, data rates, modularity). An illustration of the pixel services is given in Figure 4.6 .

Data coming from the FE chips is prepared by the MCC and transmitted electrically to the Patch Panel 0 (PP0). The PP0s are located on the Service Quarter Panels (SQPs), which are installed at both ends of the pixel detector. They hold systems for the transmission of electrical power, optical data and cooling fluid.

The electrical signal is converted into an optical one on the optoboards, which are connected to the PP0s. Each obtoboard receives data from either 6 disk modules or one half stave holding 6 or 7 pixel modules.

Vertical-Cavity Surface-Emitting Lasers (VCSEL) are used for the transformation of the electrical signal into an optical. VCSELs need very low currents for a strong optical signal and they have fast rise and fall times. The optical signals transmitted by the VCSELs are converted back into an electrical signal by PiN diodes located on the optoboards and the BOCs.

Each module uses individual fibres for the transmission of data. One fibre per module is used as the down-link, which is used for trigger and clock commands as well as configuration data. The up-link transmits event and configuration read-back data. The occupancy of the modules 
depends on their distance to the interaction point. Thus, one or two fibres are used as the up-link depending on the bandwidth requirements of the corresponding module.

Bandwidth requirements were estimated by simulations of the pixel readout system. The necessary readout rate per module was found to be $160 \mathrm{Mb} / \mathrm{s}$ in layer $0,80 \mathrm{Mb} / \mathrm{s}$ in layer 1 and $40 \mathrm{Mb} / \mathrm{s}$ in layer $2[35]$. Optoboards for the disks, layer 1, and layer 2 hold eight down-link and eight up-link channels. Only 6 or 7 of them are used since there is one optoboard per half stave available. Optoboards for layer 0 hold 7 down-link and 14 up-link channels. Two up-link channels per module in layer 0 are used with a rate of $80 \mathrm{MB} / \mathrm{s}$ in order to achieve the required readout rate.

The two most important components of the optical readout system are the Back Of Crate Card (BOC) at the off-detector side and the optoboard at the detector side.

The connection between the optoboard and the BOC is established by optical fibres. Two different types of cables are used. Radiation hard cables with lower bandwidth are used close to the on-detector part. Due to the smaller particle fluence, cables with higher bandwidth but smaller radiation tolerance are used outwards.

Each BOC is connected to one Read Out Driver (ROD). One important task of the RODs is to reroute data coming from the detector modules to the ATLAS Read Out System (ROS). The ROS system consists of PCs that store the events temporarily until events are accepted or rejected by the L2 trigger.

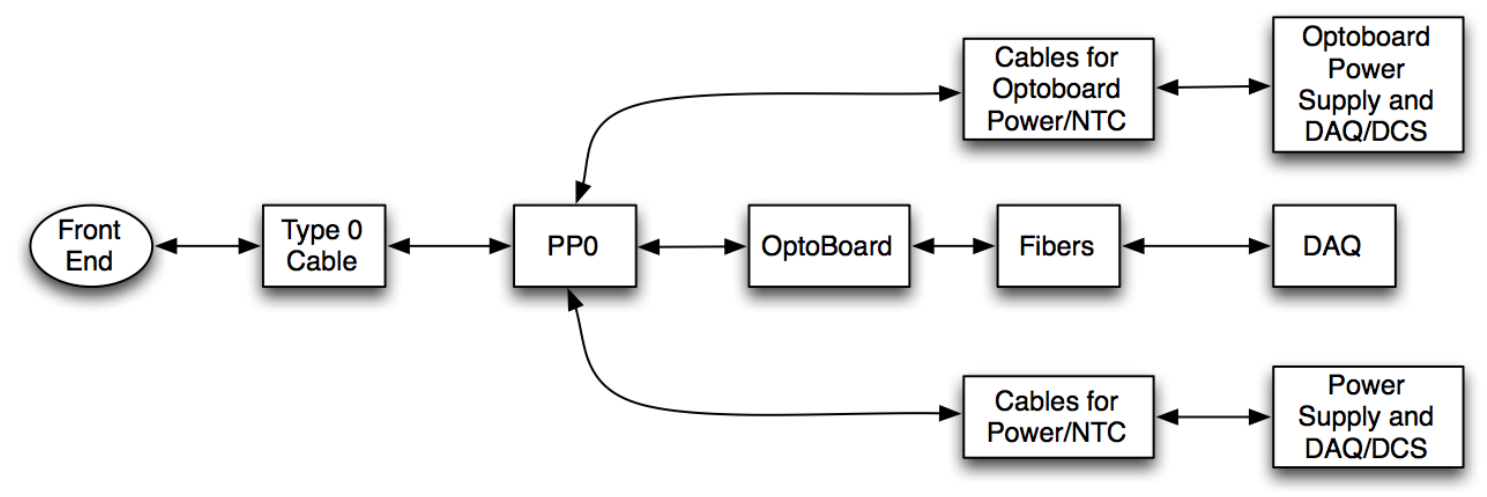

Figure 4.6.: Service structure of the ATLAS pixel detector [41]. 



\section{Upgrades of the Pixel Detector}

\subsection{Introduction}

The impact of radiation damage became increasingly visible between 2008 and 2013 while the ATLAS pixel detector was in operation. In order to counteract the loss in efficiency, a new detector layer has been developed and has been installed during the first long shutdown (LS1). The Insertable B-Layer (IBL) [43, 44] is placed between the beamline and layer 0 and thus will become the new innermost detector layer.

In addition, the current Service Quarter panels are replaced with new ones. The new Service Quarter Panels (nSQPs) are installed in order to reactivate a large fraction of the disabled modules in the pixel detector. More detailed specifications of IBL are given in Section 5.2, while the nSQPs are described in Section 5.3 .

\subsection{Insertable B-Layer}

\subsubsection{Motivation}

The pixel detector was designed as a three layer system. As the innermost detector system it is exposed to a high level of radiation and it is a crucial system for the identification of jets originating from b-quarks. The increasing impact of radiation damage and the growing number of disabled modules have an increasing effect on the detector performance. As it is discussed in Chapter 1 1 , it is very important to ensure a good b-tagging performance in order to be able to discover new phenomena. Thus, it was planned to replace the innermost layer. The original plan was to replace layer 0 after approximately 5 years of operation. First studies for a replacement of layer 0 started in 2008 with the goal of finding the best solution.

It would have been necessary to extract and disassemble the whole pixel detector in order to replace layer 0 , which is a delicate and potentially risky procedure. Furthermore, the precision of the track reconstruction in ATLAS can be improved by adding a new additional detector layer with a decreased radius, which is very important as mentioned. Fortunately the beam width at the LHC is smaller than initially expected, which made it possible to decrease the size of the beam pipe in the ATLAS detector. Thus it was decided to add a new layer to the detector instead of replacing the innermost one.

Originally it was planned to install the new layer during the shutdown in 2016, but the new LHC schedule made it necessary to change the plan and install IBL during the long shutdown in $2013 / 2014$.

\subsubsection{IBL Layout}

An overview of IBL is given in Figure 5.1. The outer radius of the new beam pipe is $24.4 \mathrm{~mm}$ and IBL is placed at a distance of $33.4 \mathrm{~mm}$ from the interaction point. The detector layer is composed of 14 staves. The staves are tilted by $14^{\circ}$ in the $R \phi$-plane with a $20 \%$ stave to stave overlap 45, 46. There is no overlap in z-direction due to space constraints. The IBL support 
structure holds the cooling pipes, it is composed of carbon fibre and is filled with a low density carbon foam. This reduces the radiation length to $60 \%$ of the radiation length of the b-layer (layer 0).

Each stave holds 20 modules. Two different types of modules are used in the IBL, 12 double chip and 8 single chip modules. High or low voltage channels always provide power for two double chip or four single chip modules.

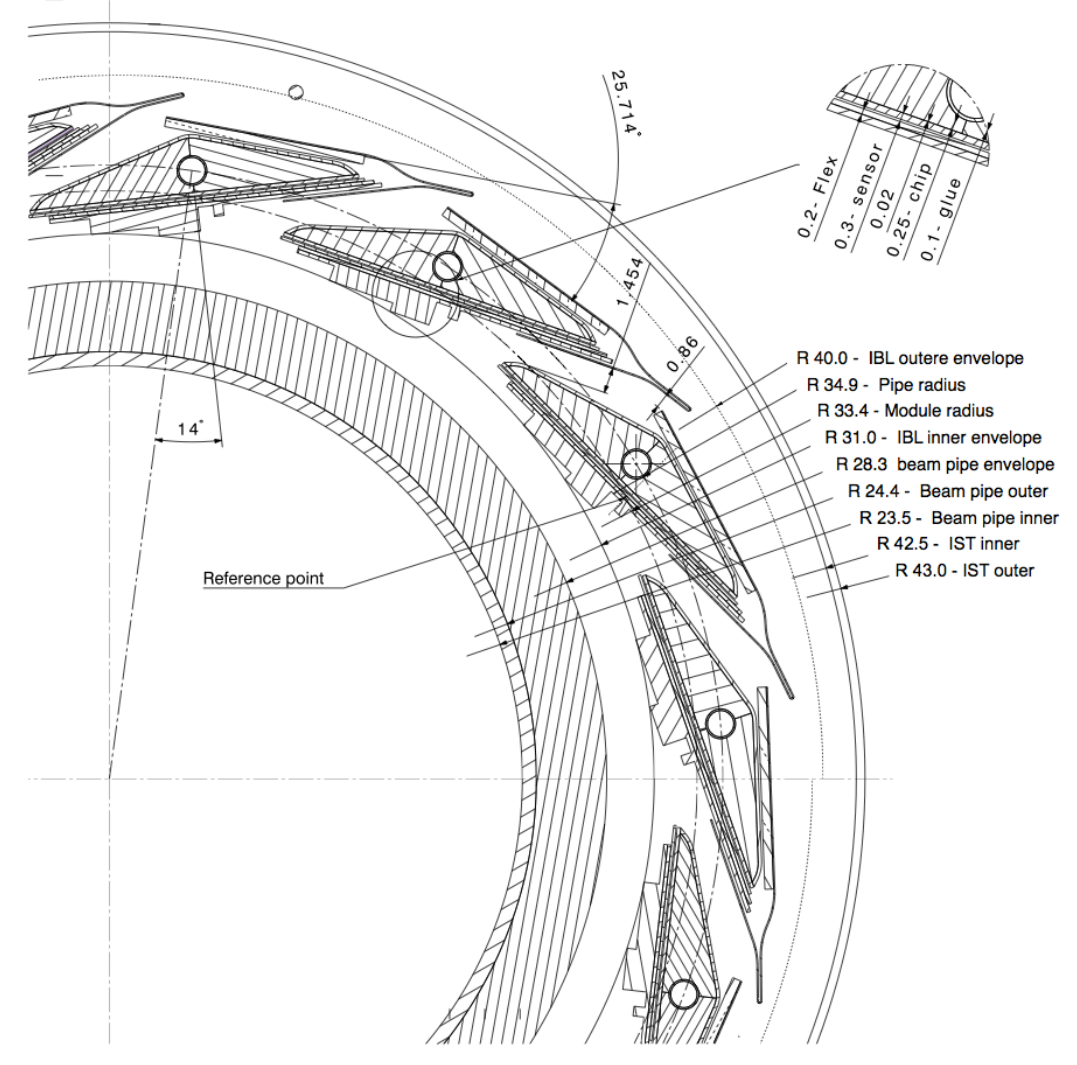

Figure 5.1.: Profile of the IBL and the new beam-pipe 45.

\subsubsection{IBL Modules}

IBL modules use hybrid technology and the basic layout is thus similar to the one of the pixel detector described in Section 4.3. Modules consist of three main components, a flexible printed circuit, the silicon sensor and the FE chips. The electrical connection between the sensor and the FE chips are realised with bump-bonds. New FE chips have been developed and the sensor design has been altered in order to cope with the high occupancy.

The IBL layer consists of two different types of modules, one using planar silicon sensors, the other 3D silicon sensors. Planar pixel sensors are used in double chip modules [47, while 3D sensors are used in single chip modules [48]. Figure 5.2 shows the two module types, their position on the stave as well as a cross-section of the stave. $75 \%$ of the sensor surface is covered by planar sensors which are placed in the centre of the stave, while the remaining $25 \%$ are covered by $3 \mathrm{D}$ modules located at both ends of the stave. 


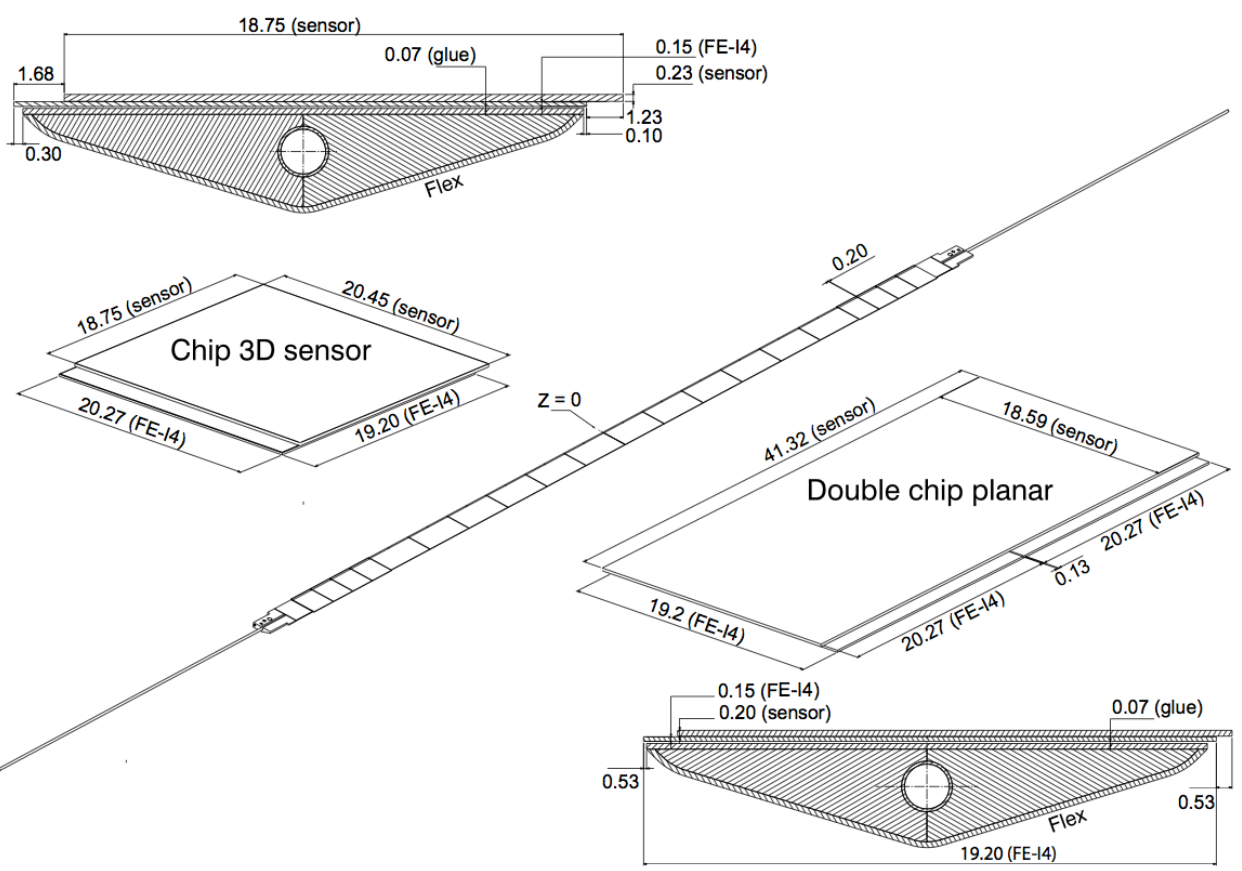

Figure 5.2.: Drawing of the IBL modules and the stave. The position of the planar and 3D sensors on the stave is indicated 45$]$.

\section{Sensor Design}

Planar sensors use the $\mathrm{n}^{+}$-in-n design. The sensors are based on the current ATLAS pixel sensor design [49]. Planar sensors are $200 \mu \mathrm{m}$ thick and the pixel size is $50 \times 250 \mu \mathrm{m}^{2}$ [45]. Guard rings are implemented close to the cutting edge in order to decrease the potential in a controlled way.

The guard ring design has been adapted to find the slimmest possible edge design that still allows safe depletion [50]. The new design reduces the inactive region at the edge of the sensors significantly and thus minimizes the impact of a stave design that has no overlap in z-direction. In total, 13 guard rings are used at each cutting edge. The outermost pixel column consist of long pixels with a length of $500 \mu \mathrm{m}$. The overlap between the guard ring region and the last pixel column is $250 \mu \mathrm{m}$ wide.

In planar sensors, the pixel implants are located at the readout side and the bottom of the sensor bulk is heavily p-doped in order to create a pn-junction, while in 3D sensors the electrodes penetrate the sensor bulk perpendicular to the sensor surface. In planar sensors, the depletion depth grows vertically to the surface from one side of the sensor to the other, while in 3D sensors the depleted region grows parallel to the surface between the electrodes.

Figure 5.3 shows the difference between a planar and a 3D sensor. In a 3D sensor, the distance between the electrodes is much smaller than the sensor thickness, thus the depletion voltage is smaller. In addition, the charge collection distance does not depend on the sensor thickness and is small. This could mitigate charge trapping effects in highly irradiated sensors [51]. As mentioned previously, charge carriers are deflected by the Lorentz force in the current ATLAS pixel modules. Due to the horizontal charge drift in 3D sensors it is expected that the impact of the magnetic field is negligible in these modules [45, 52]. 

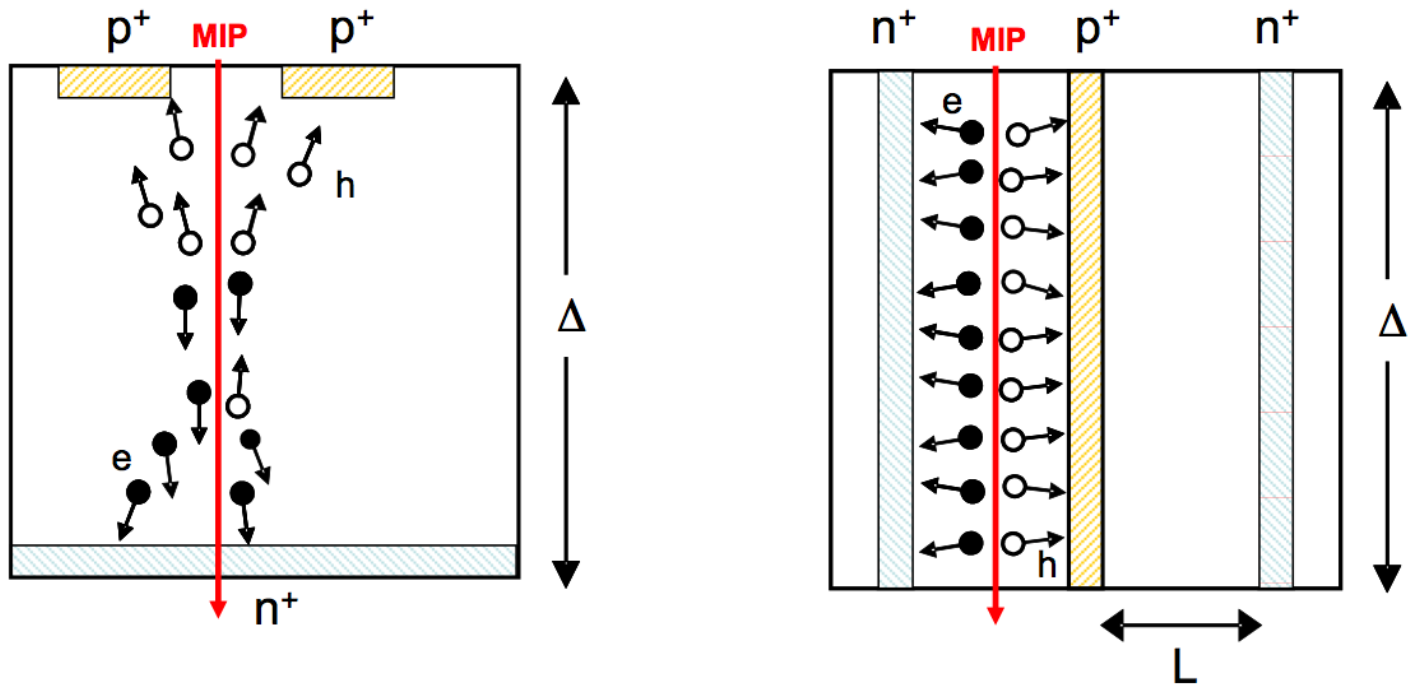

Figure 5.3.: Charge collection in a planar and a 3D pixel sensor 51].

\section{Front End Electronics}

The FE-I4 chip has a dimension of $18.8 \times 20.2 \mathrm{~mm}^{2}$. It was developed because the current FE-I3 can not fullfill the requirements for radiation hardness and high occupancy in the IBL due to the small radius. The chip that is intended for use in IBL is the Front End I4B chip (FE-I4B). It is a revised version of the FE-I4A chip. A list of the changes and a detailed description of the FE-I4B chip is given in the Note [53].

An FE-I4 chip holds an amplifier and a discriminator with independently adjustable threshold per pixel. A big advantage is, that the hits are stored locally in pixel buffers which allows faster data transmission because it is not necessary to transfer the data to a storage unit at the periphery. The FE-I4 chip saves the ToT with a 4-bit precision as well as the firing time of each discriminator. Each hit can be saved for up to 255 clock cycles (nominally $40 \mathrm{MHz}$ ). In this time interval the transmission of the information to the off detector side can be initiated by a trigger signal.

\subsection{New Service Quarter Panels}

\subsubsection{Motivation}

Signs of degradation were already visible in 2010 at the level of services. A large number of dead modules during operation were caused by the failure of several optical transmitters on the offdetector side. Fortunately it is possible to replace these transmitters on a regular basis. However, in 2011 the situation became worse and more modules needed to be disabled permanently due to failures in non reachable locations. The sensitive optical transmitters at the on-detector side are located in the SQPs and can not be replaced without removing the whole pixel detector from the ATLAS detector. In summer 2011, the new Service Quarter Panel project was initiated in order to prepare for the possibility of a high failure rate of optical transmitters at the on-detector side. The goal of the nSQP project is to move the optical transmitters from the unreachable on-detector location to a position where they can be serviced on a regular basis. 
In the meantime the failure rate increased, however, no evidence was present for optical transmitter failures as they were observed on the off-detector side. It was decided to complete the nSQP construction anyway in order to be able to install the new system during the long shutdown in 2013/2014 (LS1). Due to the problematic rate of failing modules, a series of module defect extrapolations was created in 2012 and the results were presented in January 2013 (see Chapter 7).

Finally, the pixel community and the ATLAS Collaboration made the decision to install the nSQPs.

\subsubsection{New Pixel Services}

As mentioned previously the main goal of the $\mathrm{nSQP}$ project is to extend the electrical readout system of the pixel detector in order to move the optoboards to an accessible location. The optoboards are located in the SQPs at a distance of about $1 \mathrm{~m}$ from the pixel detector (see Section 4.4). The nSQP system moves the optoboards by 6.6 meters 41 . Figure 5.4 shows a general overview of the new pixel services.

The electrical signal in the SQP is not strong enough to simply extend the electric cables. Thus, a copy of the old SQP system was made and the optical readout was replaced. Instead of the optoboards, E-boards are used which hold an electrical repeater chip to amplify the signal. The E-boards have the same form factor and electrical connections as the optoboards. Electrical cables and the ER-bundle are used to extend the electric readout to the new optoboard position at the detector end plate.

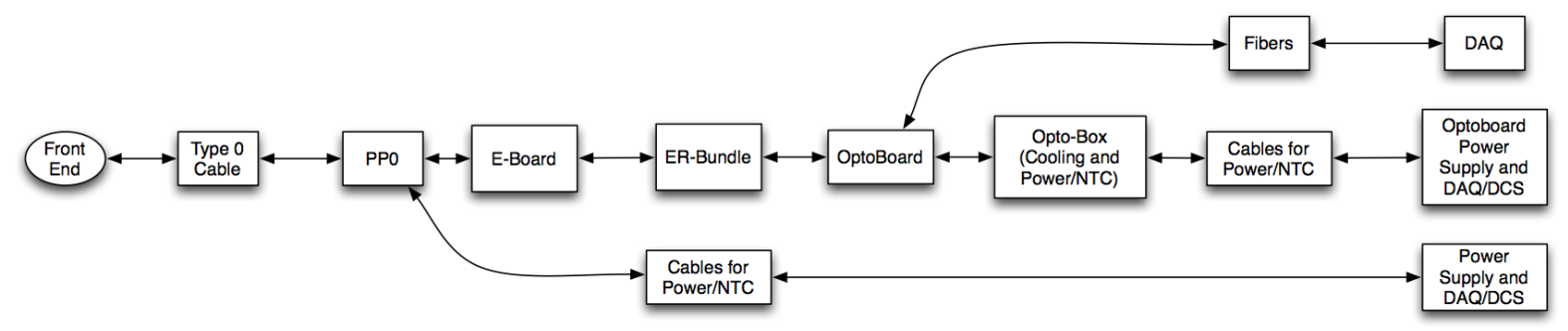

Figure 5.4.: New Service structure of the ATLAS pixel detector [41].

Currently the readout system is limited to a data rate per module of $160 \mathrm{Mb} / \mathrm{s}$ in layer 0 . The bandwidth in layer 1 is $80 \mathrm{Mb} / \mathrm{s}$ and $40 \mathrm{Mb} / \mathrm{s}$ in layer 2 as mentioned previously in Section 4.4. The bandwidth limitations might prove to be a problem in the future for runs with very high luminosity. The installation of the nSQP system offers the opportunity to increase the bandwidth per module in layer 1 to $160 \mathrm{Mb} / \mathrm{s}$ allowing the detector to cope with higher occupancies. 



\section{Monitoring Radiation Damage in the Pixel Sensor}

\subsection{Introduction}

Radiation damage effects in the ATLAS pixel detector become increasingly visible with the advancing run time of the LHC. The effective depletion voltage and the depletion depth are key values for the monitoring of radiation damage in the pixel detector. Continuous monitoring of these values is crucial to ensure that the expected performance of the pixel detector is met until it can be replaced.

Due to the sensor design it is expected that the depletion voltage decreases up to the point of type-inversion, after which the sensor bulk is effectively p-type silicon. After type-inversion the depletion voltage increases constantly with the accumulated radiation dose. It is possible to increase the standard voltage of operation from $150 \mathrm{~V}$ up to $600 \mathrm{~V}$. The pixel sensors are designed to be operated partially depleted as soon as the depletion voltage exceed the maximum operation voltage.

Monitoring of radiation damage is performed by a range of methods. Before type-inversion dedicated cross-talk scans were used, which were taken regularly during the maintenance stops of the LHC. Results of the cross-talk scans in the innermost layer reveal a general trend of reduction in the effective depletion voltage between summer 2011 and summer 2012. At this time the effective depletion voltage was very close to its expected minimum and indicated the start of type-inversion in the pixel sensors.

The cross-talk method relies on the growth of the depleted region from the back side of the sensor and is thus not applicable after type-inversion. Two track based depletion depth measurements have been developed in order to be able to monitor the development of radiation damage after type-inversion using collision data. Both methods are fully data driven and do not rely on Monte Carlo data.

Dedicated depletion depth scans confirmed the start of type-inversion in the innermost layer in summer 2012. Additional scans in fall 2012 also confirmed type-inversion in layer 1. Furthermore, the scans reveal a decrease in depletion depth over time, if the bias voltage is fixed to a value that is below the effective depletion voltage.

Finally, a method has been developed to measure the effective depletion voltage after typeinversion. It uses the results of the track based depletion depth scans. Measurements after type-inversion reveal an increase of the effective depletion voltage in the innermost layer since summer 2012 and for layer 1 since fall 2012, as expected.

The accumulated radiation dose in the outermost detector layer is considerably smaller and is thus only included in the monitoring from time to time until radiation damage effects become more visible.

A general overview of the effects of radiation damage in silicon is given in Section 6.2, while the local coordinate system of the pixel detector is explained in Section 6.3. The cross-talk method is explained in Section 6.4. Track based depletion depth measurements are presented in Section 6.5 and 6.6. followed by the measurement of the effective depletion voltage after 
type-inversion in Section 6.7 .

\subsection{Radiation Damage in Silicon}

Charged particles that travel through silicon lose their energy primarily by excitation of electrons as discussed in Chapter 2. However, additional types of energy loss also occur such as the displacement of lattice atoms.

Radiation damage in silicon sensors can be separated into two categories, bulk damage and surface damage. Surface damage comprises all defects that are located close to the surface of the silicon crystal as well as in the interface area between the silicon and passivation layer. Bulk defects are located deeper inside the silicon sensor. The creation of bulk damage is described by the so called Non Ionising Energy Loss (NIEL) hypothesis.

This section focuses on the creation and the impact of bulk damage, because it is the deciding factor for the performance of a silicon sensor. The microscopic effects that lead to radiation damage in silicon are discussed and the macroscopic impact which has an influence on the operation of the pixel detector is summarised.

\subsubsection{Bulk Damage}

A passing particle can knock silicon atoms off of their lattice position and thus is able to create defects in the silicon crystal. The displacement of atoms in the lattice leads to the creation of Vacancy-Interstitial (V - I) pairs (Frenkel defect). The minimum energy necessary to create a Frenkel pair is approximately $25 \mathrm{eV}[54]$. The energy of a neutron creating such a point defect has to be $175 \mathrm{eV}$ or more, while electrons need at least an energy of $260 \mathrm{keV}$ to create such displacements [55].

Silicon atoms that are displaced by interactions with a passing particle are called Primary Knock-on Atoms (PKAs). At lower particle energies no further defects are created, however, if the PKA has received sufficient energy it travels through the crystal and loses its energy by ionisation and the creation of further Frenkel defects. In addition to single point defects, high energetic PKAs also create clusters with a high density of defects in a relatively small area. Clusters are usually created when the silicon atom already has lost most of its energy. Thus, they are in general located at the end of the PKA's path. For very high energies, it is possible that point defects created by the PKA also possess enough energy to create additional defects. These atoms are called secondary knock-on atoms.

A PKA with an energy of $50 \mathrm{keV}$ can create up to 1000 additional defect pairs, while approximately 600 of the defects recombine again [56]. A simulation of a PKA creating further defects is shown in Figure 6.1. The exact distribution varies from event to event. The example shows a structure created by one PKA with an energy of $50 \mathrm{keV}$. This is a typical energy for $1 \mathrm{MeV}$ neutron scattering in silicon. A tree-like structure and several sub-clusters are clearly visible. Sub-clusters are created by secondary knock-on atoms with lower energy. If the PKA energy is increased above $50 \mathrm{keV}$ a larger tree with several branches can be observed [57.

Particles that are charged and neutral particles interact differently with matter. Charged particles can interact electrically with the nuclei which increases the probability of the creation of point defects. Neutrons, on the other hand, only interact via the nuclear force and thus create more clusters. However, the difference is evened-out for highly energetic particles, because secondary knock-on atoms have enough energy to create additional defects in the silicon crystal, as mentioned previously 22 . 


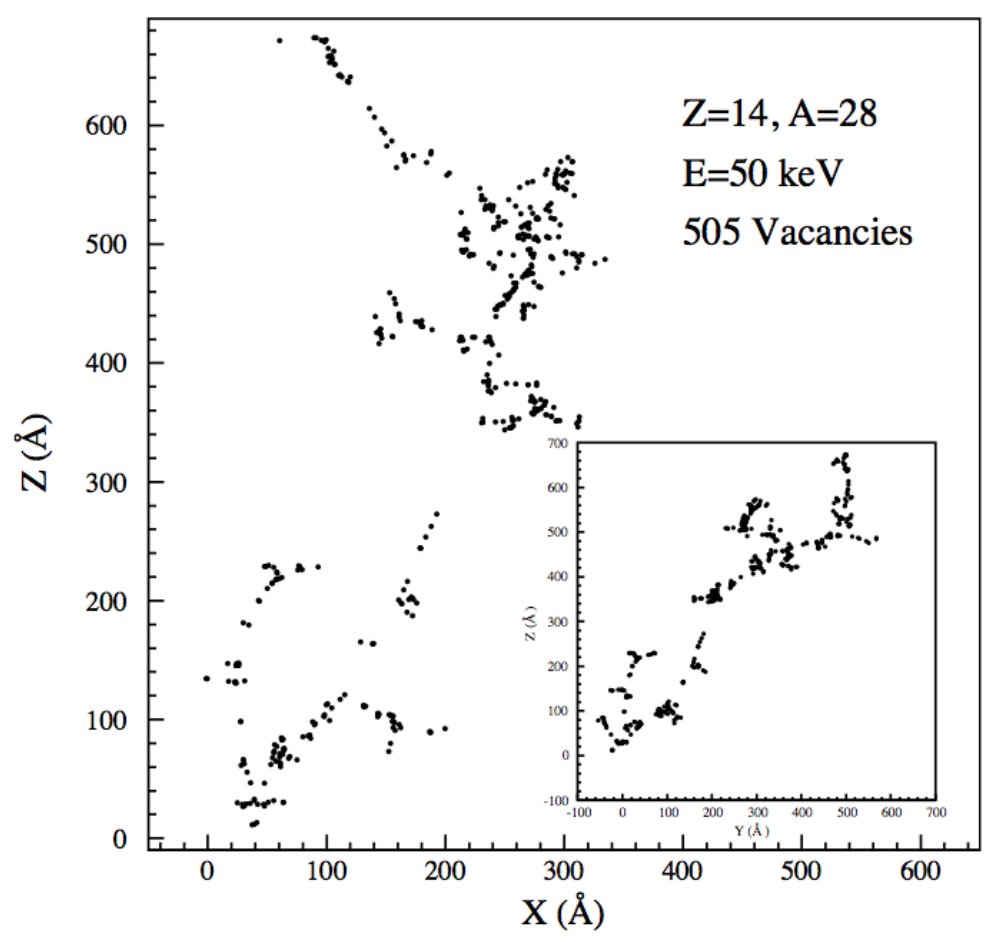

Figure 6.1.: Simulation of defects in silicon created by a single PKA with an energy of $50 \mathrm{keV}$. The transverse projection is shown in the inset. Point defects as well as cluster defects are visible [57].

The NIEL scaling technique is used if a comparison of samples irradiated with different types of particles is desirable. The impact of the radiation damage of highly energetic particles is scaled to the damage of $1 \mathrm{MeV}$ neutrons as a reference. A hardness factor $\kappa$ is introduced and used together with the total fluence, $\phi_{p}$, of the corresponding particles in order to calculate the neutron equivalent fluence: $\phi_{\mathrm{eq}}=\kappa \phi_{p}$. A short summary of the NIEL scaling technique is given in [55], while information on the calculation of the hardness factor can be found in [58].

The created point defects are mobile and thus are able to move through the silicon crystal and many of them recombine again as mentioned earlier. However, stable defects are also formed. Various types of stable defects can be created - it is for example possible that defects react with each other or with impurity atoms. These stable defects as well as defect clusters can be electrically active and are thus the source of the measurable radiation damage effects in silicon.

Many combinations of point defects are possible. A few examples of defect formations are shown in Figure 6.2. A-centres $(V-O)$ are formed if a vacancy and an oxygen atom are joined, while E-centres are created if a vacancy and a phosphorus $(V-P)$ or a vacancy and a Boron atom $(V-B)$ are joined into a stable defect. Two vacancies can create a di-vacancy, while cluster defects consist of a large amount of crystal defects. Cluster defects show a distinct behaviour and thus they cannot be described as a series of independent point defect.

A lot of progress has been made in identifying various additional microscopic defects in silicon and their relationship to the macroscopic effects. Detailed information on the work on defect characterisation can for example be found in [59, 60, 61].

The formation of point defects and defect clusters has a large impact on the performance of the silicon sensor. Defects with energy levels in the centre of the band gap act as recombination- 
generation centres, because it is likely that simultaneous capture or thermal emission of electrons and holes occurs. Shallow defects which have an energy level close to the valence or conduction band on the other hand act as trapping centres due to the fact that they can capture free charge carriers temporarily. The trapped charge carriers are re-emitted after a time delay. Finally, some of the defects also posses energy levels that are similar to the ones of acceptors and donors. All of these defect characteristics lead to a visible change in the behaviour of silicon sensors, as is explained in the following.

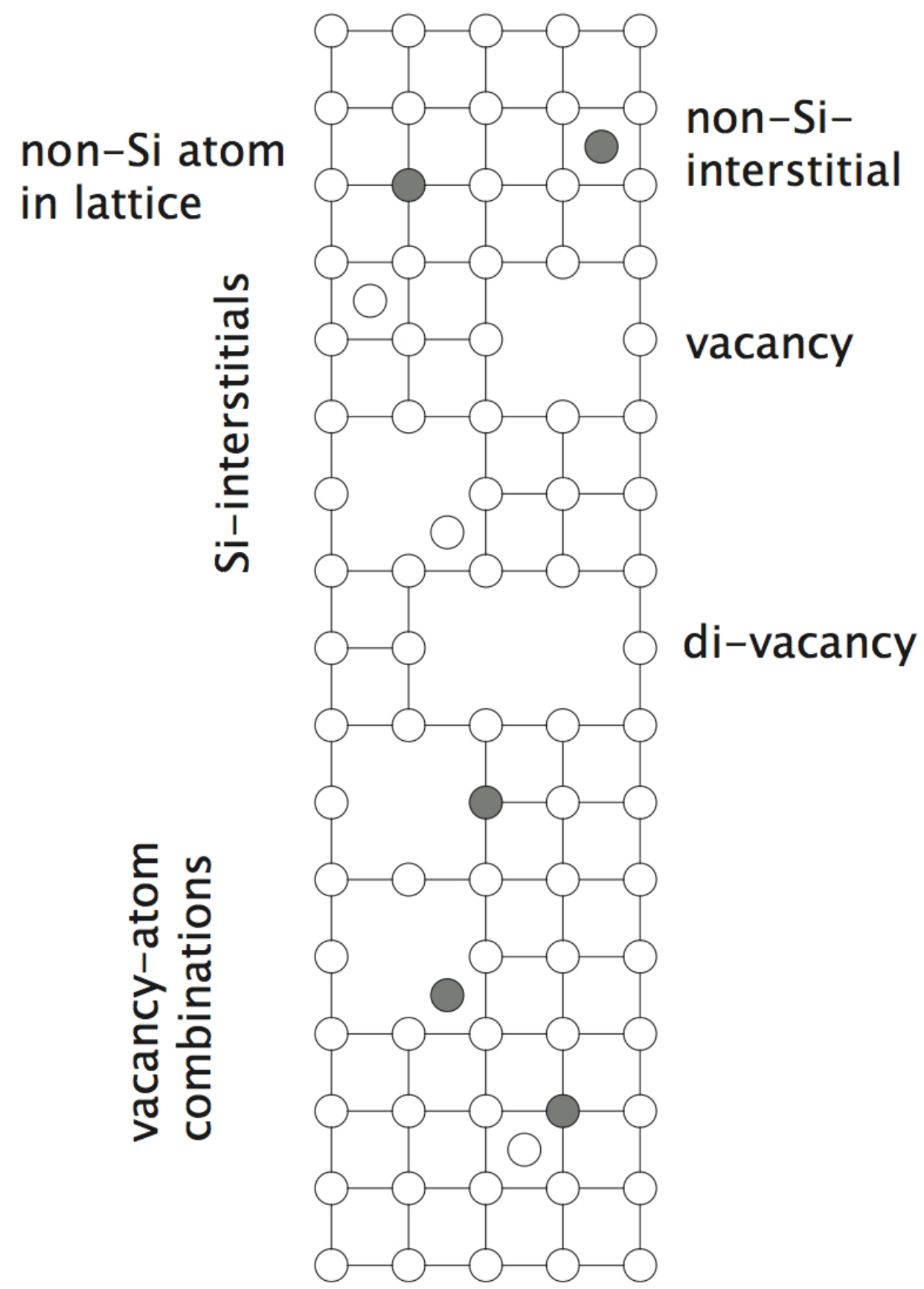

Figure 6.2.: Possible crystal defects introduced by non ionising energy losses 36,56 


\subsubsection{Impact on Sensor Performance}

The microscopic defects accumulate over time and have an increasing impact on the sensor performance, which ultimately limits the lifetime of silicon sensors used in high energy experiments. The properties of the sensor are altered in three main ways that are important for detector operation:

- Recombination-generation centres increase the leakage current, which affects the signal to noise ratio, the power consumption and the cooling system of the pixel detector.

- Defects change the effective doping concentration of the sensor, which has an impact on the depletion voltage. Overall more p-type than n-type defects are created, which leads to type-inversion of the sensor.

- Trapping centres cause a decrease in the charge collection efficiency. A degrading hit efficiency is the result, which in the end has a negative impact on the $b$-tagging performance and track resolution of the detector.

The impact of radiation damage is not constant after irradiation has stopped, because the created defects can anneal. Silicon interstitial and vacancies can recombine as already mentioned, however, stable defects are also able to transform and become inactive, which is beneficial for the sensor performance. Inactive defects can also be reactivated and thus increase the impact of radiation damage again.

\section{Leakage Current}

An example of the increase in the leakage current per unit of volume $\Delta I / V$ in several types of silicon is shown in Figure 6.3. In these measurements the leakage current was measured before and after irradiation in order to estimate its increase. An important factor that needs to be taken into account for a precise measurement is the dependence of the leakage current on the temperature. In this case all current measurements were normalised to a reference temperature of $20^{\circ} 62$.

The increase in the measured leakage current is proportional to the particle fluence and it is clearly visible that it does not depend on the silicon type. The relationship between the leakage current and the collected fluence can thus be written as 62$]$ :

$$
\Delta I=\alpha \phi_{\mathrm{eq}} V,
$$

where $V$ is the sensor volume and $\alpha$ is the current related damage rate.

Annealing effects are described by the current related damage rate, which is therefore a function of time, $\alpha(t)$. The value of $\alpha$ was found to decrease over time 62]. From this it follows that annealing effects are exclusively beneficial for the leakage current. Several models exist to describe the impact of annealing, however, in general the long term behaviour of $\alpha$ is described by a fit function 62 :

$$
\alpha(t)=\alpha_{I} \exp \left(-\frac{t}{\tau_{I}}\right)+\alpha_{0}-\beta \cdot \ln \left(\frac{t}{t_{0}}\right)
$$

where $\alpha_{I}, \alpha_{0}$ and $\beta$ are fit parameters and the time $t_{0}$ is set to 1 minute. 


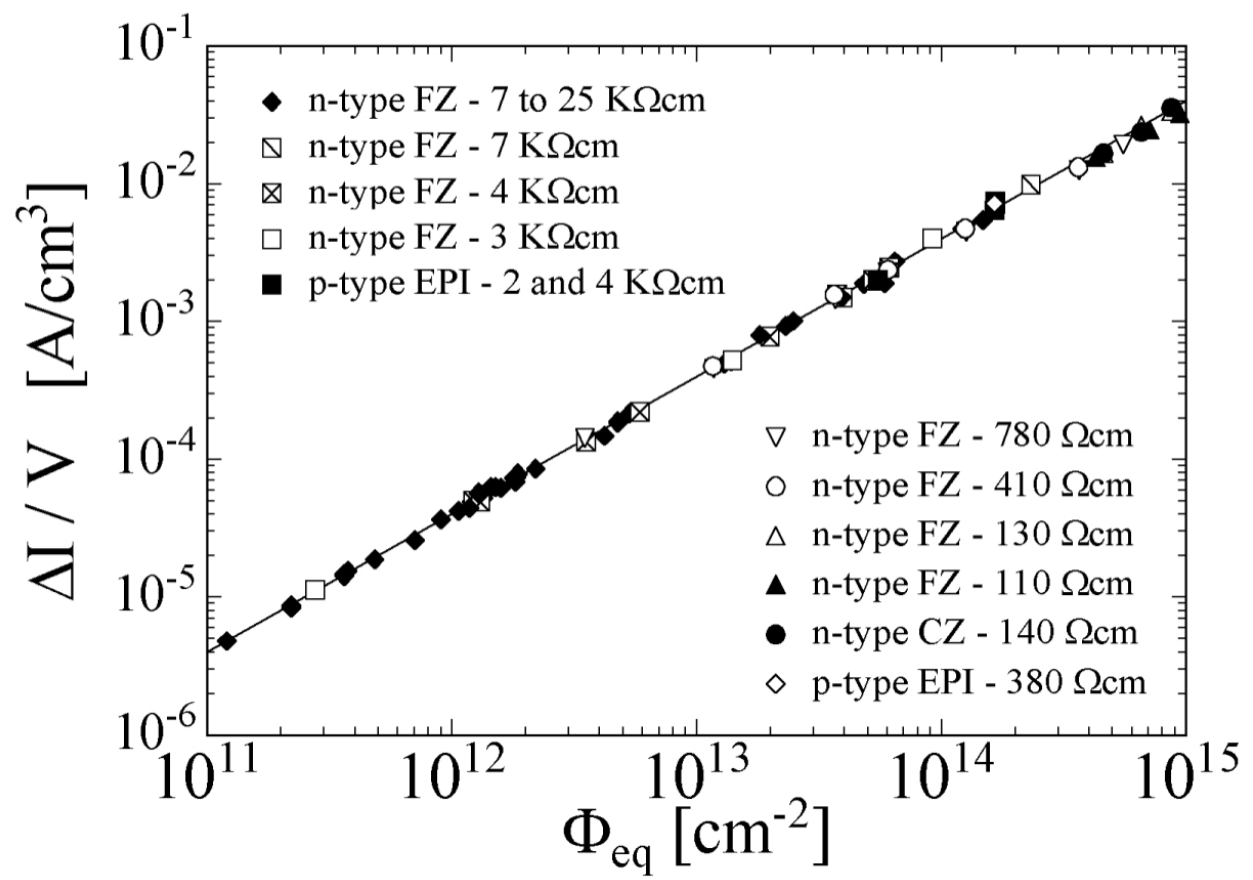

Figure 6.3.: The leakage current as a function of the neutron equivalent fluence shown for different types of silicon. The measurements were taken after heating the sensor to $60^{\circ}$ degrees for 80 minutes in order to be comparable to other measurements 62 .

The time constant $\tau_{I}$ is based on the Arrhenius equation, which describes reaction rates as a function of the temperature. It is expressed as:

$$
\frac{1}{\tau_{I}}=k_{0 I} \cdot \exp \left(-\frac{E_{I}}{k_{B} T_{a}}\right),
$$

with two fit parameters $k_{0 I}$ and $E_{I}$. $T_{a}$ is the temperature at which the silicon sensors were stored during the annealing period. $T_{a}$ is in general different to the temperature at which the leakage current is measured.

According to 62 the parameters of the fit have been determined to be:

$$
\begin{aligned}
\alpha_{I} & =(1.23 \pm 0.06) \cdot 10^{-17} \mathrm{~A} / \mathrm{cm} \\
k_{0 I} & =1.2_{-1.0}^{+5.3} \cdot 10^{13} \mathrm{~s}^{-1} \\
E_{I} & =(1.11 \pm 0.05) \mathrm{eV} \\
\beta & =(3.07 \pm 0.18) \cdot 10^{-18} \mathrm{~A} / \mathrm{cm} \\
\alpha_{0} & =-(8.9 \pm 1.3) \cdot 10^{-17} \mathrm{~A} / \mathrm{cm}+(4.6 \pm 0.4) \cdot 10^{-14} \mathrm{AK} / \mathrm{cm} \cdot \frac{1}{T_{a}}
\end{aligned}
$$

A growing leakage current increases the noise in the sensor as well as the power consumption. Due to the fact that the leakage current can reach the magnitude of $\mathrm{mA}$ in highly irradiated sensors, it can cause thermal runaway. The heat dissipation caused by a current of this magnitude is large enough to increase the leakage current, leading to an even higher heat dissipation. This self amplifying loop can finally destroy the sensor. Thus, it is crucial to ensure proper cooling of silicon detectors during operation. 


\section{Effective Doping Concentration and Depletion Voltage}

Acceptor-like defects and donor removal lead to a change in the effective doping concentration in the silicon bulk. Donor removal can occur if foreign donor atoms such as phosphorus are combined with a vacancy and thus create a new defect which has different electrical properties. An example measurement for an initially n-type doped sensor is shown in Figure 6.4. The effective doping concentration as well as the depletion voltage decrease at the beginning up to the point of type-inversion. Afterwards the silicon is effectively p-doped and the doping concentration as well as the depletion voltage increases continuously with the particle fluence.

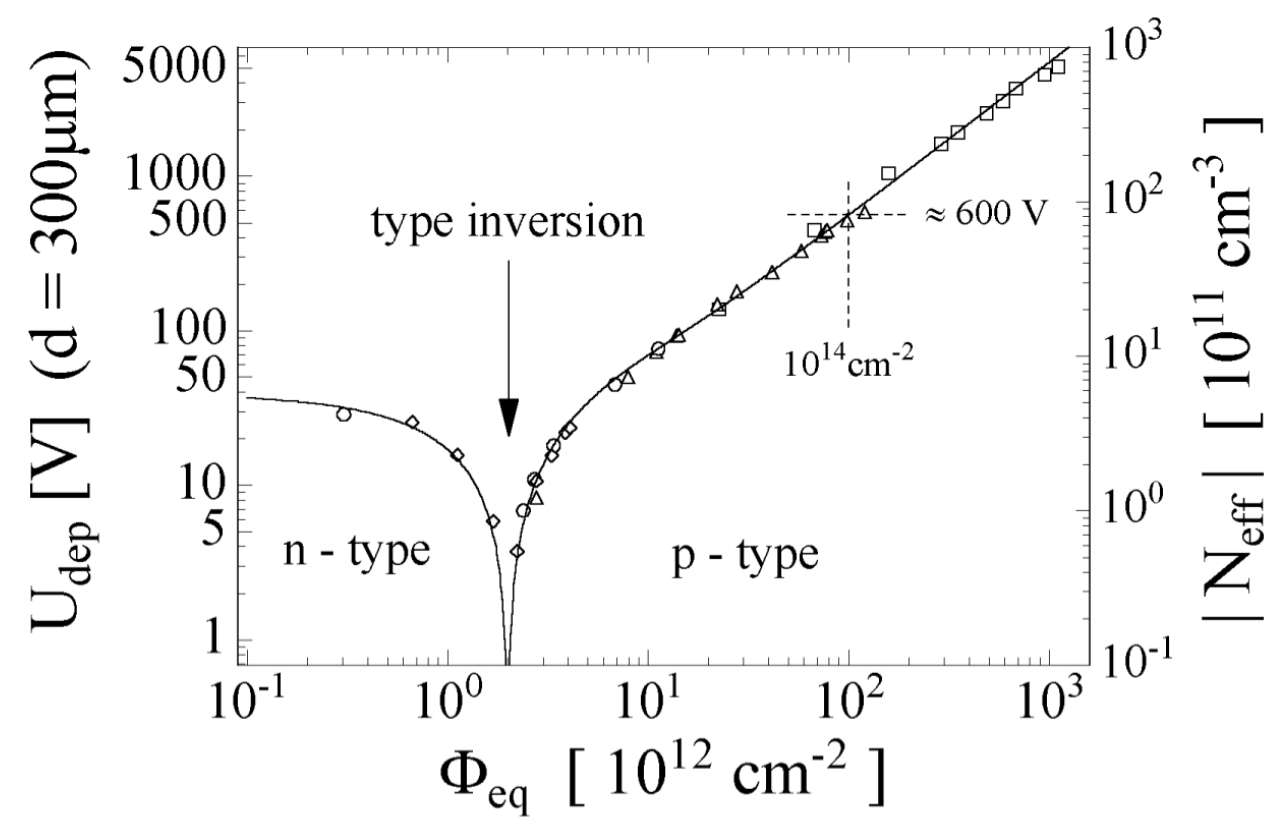

Figure 6.4.: Change in effective doping concentration / depletion voltage as a function of the neutron equivalent fluence. The measurements were taken directly after irradiation 56 .

According to 62 , the change in the doping concentration can be quantified with the following formulas (Equations 6.4- 6.10). Several parameters of these equations are again estimated via fits. The reader is also referred to 62 for the estimation of the fit parameters that are mentioned in this paragraph. Furthermore, an additional short summary of the model and its parameters can also be found in [22]. The effective doping concentration $N_{\text {eff }}\left(\phi_{\text {eq }}, t\left(T_{a}\right)\right)$ is described as:

$$
N_{\text {eff }}\left(\phi_{\text {eq }}, t\left(T_{a}\right)\right)=N_{\text {eff }, 0}-\Delta N_{\text {eff }}\left(\phi_{\text {eq }}, t\left(T_{a}\right)\right),
$$

where $N_{\text {eff }, 0}$ is the initial doping concentration and $\Delta N_{\text {eff }}\left(\phi_{\text {eq }}, t\left(T_{a}\right)\right)$ the change in the concentration induced by irradiation. The effective doping concentration depends on the time and the fluence, where the time dependence is a function of the temperature $T_{a}$. The induced change $\Delta N_{\text {eff }}\left(\phi_{\text {eq }}, t\left(T_{a}\right)\right)$ can be written as:

$$
\Delta N_{\text {eff }}\left(\phi_{\text {eq }}, t\left(T_{a}\right)\right)=N_{C}\left(\phi_{\text {eq }}\right)+N_{A}\left(\phi_{\text {eq }}, t\left(T_{a}\right)\right)+N_{Y}\left(\phi_{\text {eq }}, t\left(T_{a}\right)\right) .
$$

$N_{C}$ represents the stable defects and thus only depends on the fluence, $N_{A}$ is the part characterising the short term annealing, and $N_{Y}$ is the reverse annealing component of the formula. 
The function for the stable damage consists of two parts. An exponential function leads to a decrease in the effective doping concentration until the minimum $N_{C 0}$ is reached. The decrease can be interpreted as donor removal, while the second term is linear and describes the creation of acceptor-like states in the silicon sensor:

$$
N_{C}=N_{C 0}\left(1-\exp \left(-c \phi_{\mathrm{eq}}\right)\right)+g_{c} \phi_{\mathrm{eq}} .
$$

The values of the parameters $N_{C 0}$ and $c$ depend on the type of silicon that is used. Some values are listed in [62]. The factor $g_{c}$ is only slightly dependent on the silicon material and the average is $g_{c}=(1.49 \pm 0.04) \cdot 10^{-2} \mathrm{~cm}^{-1}$.

The short term annealing component is described as:

$$
N_{A}=\phi_{\mathrm{eq}} \sum_{i} g_{a, i} \exp \left(-\frac{t}{\tau_{a, i}}\right)
$$

Annealing times that are very short are often not important for the operation of silicon detectors. Additional information on short annealing times is for example presented in [56]. Therefore all time constants except the longest decay time are generally neglected. This reduces Equation 6.7 to a single exponential function. The parameter of the amplitude is estimated as $g_{a}=(1.49 \pm$ $0.04) \cdot 10^{-2} \mathrm{~cm}^{-1}$, while the time constant has a dependence of:

$$
\frac{1}{\tau_{a}}=k_{0 a} \cdot \exp \left(-\frac{E_{a}}{k_{B} T_{a}}\right)
$$

with $k_{0 a}=2.4_{-0.8}^{+1.2} \cdot 10^{13} \mathrm{~s}^{-1}$ and $E_{a}=(1.09 \pm 0.03) \mathrm{eV}$. Reverse annealing can also increase the change in the doping concentration in contrast to the annealing of the leakage current. The third term in Equation 6.5 describes this behaviour:

$$
N_{Y}=N_{Y, \infty}\left(1-\frac{1}{1+\frac{t}{\tau_{Y}}}\right),
$$

where $N_{Y, \infty}=\phi_{\text {eq }} g_{y}$ is the amplitude, with $g_{Y}=(5.16 \pm 0.09) \cdot 10^{-2} \mathrm{~cm}^{-1}$. The time constant is again described as:

$$
\frac{1}{\tau_{Y}}=k_{0 Y} \cdot \exp \left(-\frac{E_{Y}}{k_{B} T_{a}}\right)
$$

with $k_{0 Y}=1.5_{-1.1}^{+3.4} \cdot 10^{15} \mathrm{~s}^{-1}$ and $E_{Y}=(1.33 \pm 0.03) \mathrm{eV}$. The combination of all three components is illustrated in Figure 6.5 and the impact of beneficial and reverse annealing is clearly visible.

The evolution of radiation damage in silicon can be slowed down by inducing oxygen into the silicon sensor [63], this is the case for the sensors in the ATLAS pixel detector 33]. However, after years of operation the maximum voltage of the power supplies is nevertheless reached and the sensor cannot be operated fully depleted any longer. Due to the n-in-n sensor design that is used in the ATLAS pixel detector it is possible to operate the detector partially depleted after type-inversion. Before type-inversion, the depleted region grows from the backside of the sensor as is shown in Figure 6.6(a). Thus, it is only possible to receive a signal if the sensor is fully depleted. After type-inversion the p-n junction is located at the pixel side and therefore the depleted region grows from the readout side as shown in Figure 6.6(b). This offers the opportunity to operate the detector after the maximum voltage has been reached, even though the sensor efficiency will of course decrease, since the collected charge depends on the size of the active region. 


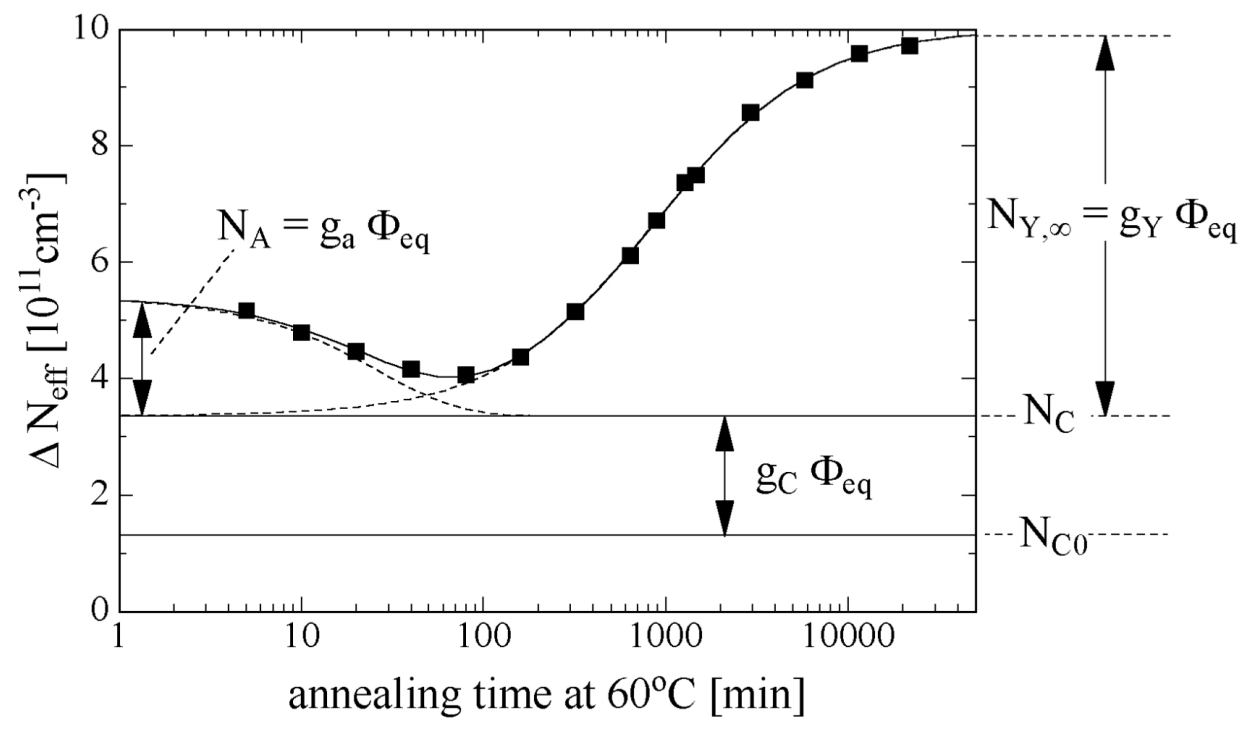

Figure 6.5.: Change in the effective doping concentration $\Delta N_{\text {eff }}$ caused by annealing effects as a function of time. The temperature was set to $60^{\circ}$ and the sample was irradiated with a fluence of $1.4 \cdot 10^{13} \mathrm{~cm}^{-2} 62$.

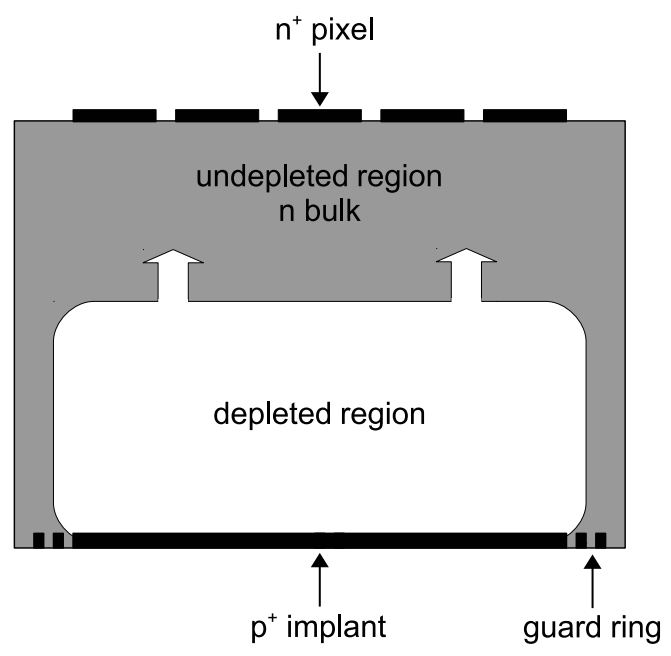

(a)

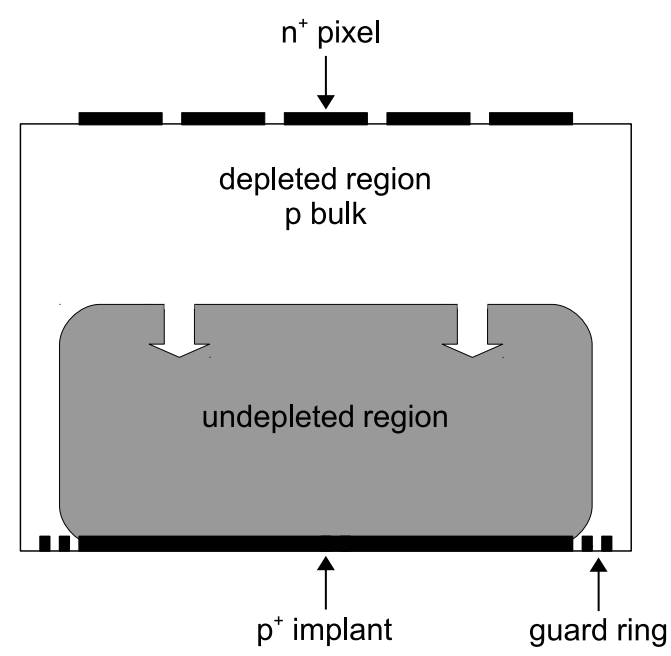

(b)

Figure 6.6.: Schematic view of a silicon sensor before (a) and after type-inversion. The depleted region grows from the backside of the sensor before and from the readout side after type-inversion. Thus, it is possible to operate the sensor partially depleted after type-inversion. 


\section{Charge Trapping}

A fraction of the free charge carriers drifting towards the electrodes in the silicon sensor are trapped for a limited time by defects. If the trapping time is longer than the time available for readout, then the charge carriers do not contribute to the signal and its amplitude will decrease. The trapping time depends highly on the type of trap, only traps with energy levels very close to the valence/conduction band might release the holes/electrons fast enough so that the charge collection is not affected [15].

Traps, and thus the charge carrier loss in the silicon sensor, are characterised by the trapping time constant $\tau^{+}(\phi)$, which describes the average time that a charge carrier stays trapped. It is inversely proportional to the fluence [56]:

$$
\frac{1}{\tau^{+}(\phi)}=\frac{1}{\tau_{0}^{+}}+\gamma \phi
$$

Both parameters have been measured and the values were found to be $\tau_{0}^{+}=0.51 \cdot 10^{6} \mathrm{~s}^{-1} \pm 12 \%$ and $\gamma=0.24 \mathrm{~cm}^{2} \mathrm{~s}^{-1}$. The quoted values are valid for electrons and holes, however, they cannot be used above a fluence of $\phi=8.8 \cdot 10^{12} \mathrm{~cm}^{-2}[56$.

Measurements that assess the radiation damage in the ATLAS pixel detector have been taken regularly until the LHC was shutdown in order to install several upgrades at the beginning of 2013. Results of the monitoring program are discussed in the following.

\subsection{Local Coordinate System}

In the following measurements a special local coordinate system is used in addition to the global coordinate system (see Section 3.3.1). The local coordinate system is designed to identify the pixel modules in the detector and the position of tracks inside single pixel modules. The module position on a stave is illustrated in Figure 6.7. The numbering starts at the centre of the stave. Modules closer to the C-Side of the cavern receive a positive number, while modules on the A-side receive negative numbers.

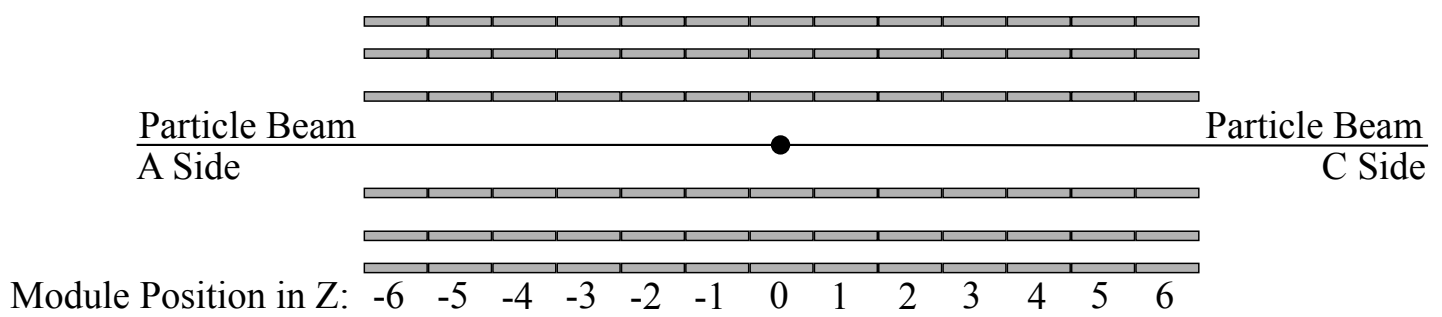

Figure 6.7.: Stave position of pixel modules in the pixel detector. The interaction point is indicated as the black point. Module numbers can be negative and positive. The closer the module is located to the interaction point the smaller is the stave number.

Figure 6.8 illustrates the local coordinate system of the ATLAS pixel modules. Row and column numbering starts at the left corner of FE chip 0. Short pixel edges are parallel to the local x-axis, while the local y-axis is parallel to the long pixel direction. The origin of the local coordinate system is the centre of each module between FE chip 3, 4, 11 and 12. The local z-axis of the module points from the backside of the sensor to the readout side. Particles travel 
usually in positive the z-direction, since the interaction point is located below the backside of the sensors. The origin of the z-axis is located at the centre of the active silicon [64].

Local incidence coordinates are defined in addition to the global spherical system. The local incidence angle in the local xz-plane is named $\varphi$. Particles that travel parallel to the local z-axis in the positive direction have a track incidence angle $\varphi$ of 0 . Tracks that are parallel to the local $\mathrm{x}$-axis have angles of $\frac{\pi}{2}$ or $-\frac{\pi}{2}$. The total range is $[-\pi, \pi]$.

The local angle of aperture $\theta$ is projected in the local yz-plane. It is measured clockwise and tracks parallel to positive $\mathrm{z}$ have an angle of 0 , while particles that travel in positive $\mathrm{y}$ have an angle of $\frac{\pi}{2}$. The total range is $\left[-\frac{\pi}{2}, \frac{\pi}{2}\right]$. An illustration of local $\theta$ is given in Figure 6.9 .

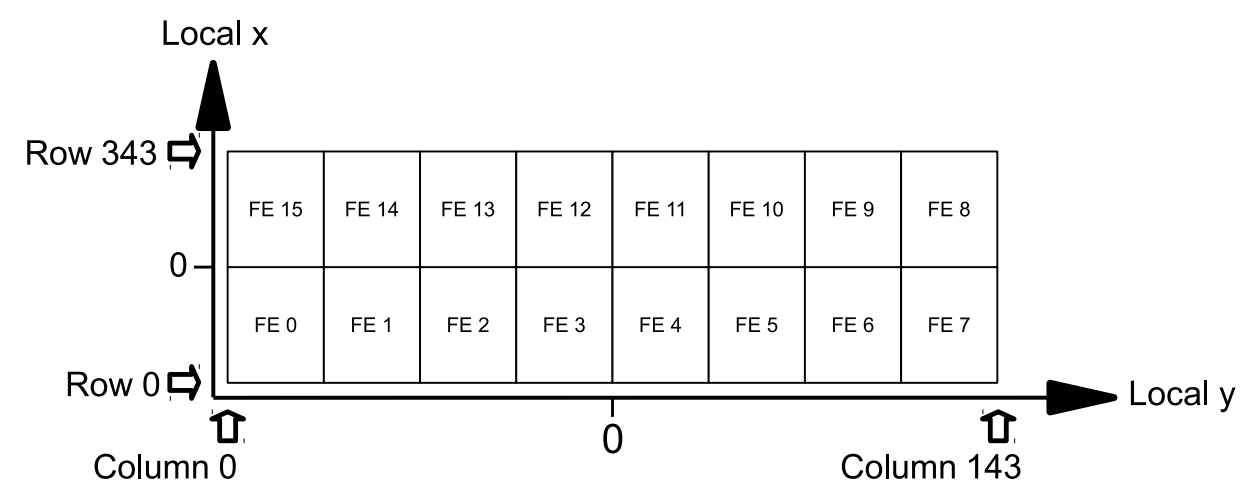

Figure 6.8.: Overview of the local coordinate system used for ATLAS pixel modules. Local y is parallel to the long pixel direction while local $\mathrm{x}$ is parallel to the short pixel direction. Local $\mathrm{z}$ points towards the readout side of the pixel sensor.

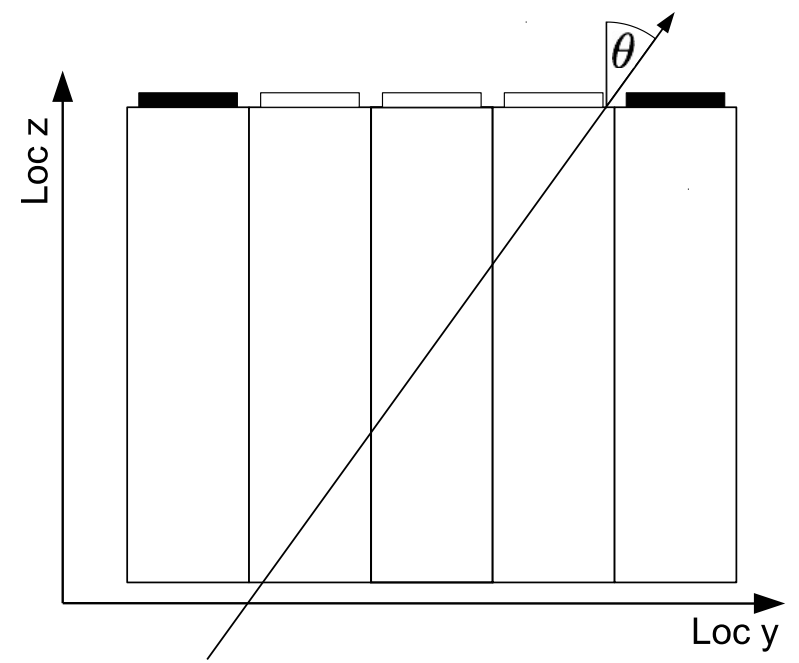

Figure 6.9.: Projection of a pixel module in the local yz-plane (not to scale). A track passing the sensor and the local angle of aperture $\theta$ are indicated. 


\subsection{Measurement of the effective Depletion Voltage before type-inversion}

\subsubsection{Measurement Method}

Before type-inversion an inter-pixel cross-talk scan is used to determine the effective depletion voltage in the ATLAS pixel modules. The method is an evolution of earlier pixel cross-talk measurements 65, 66]. First result: 11 of the effective depletion voltage measurement are presented in [67].

As long as the pixel sensor is not fully depleted a high-ohmic short exists between neighbouring pixel and free charge carriers are able to drift between adjacent pixels, while all pixels are isolated from each other in a fully depleted sensor. The change in the conductivity is used for determination of the effective depletion voltage. A pixel $n$ is chosen as the readout pixel and charge is injected in two adjacent pixels $n+1$ and $n-1$. The hit counter is increased by one if enough charge is collected in pixel $n$ to exceed its threshold. The cross-talk measurement is repeated 100 times for every pixel. At the bias voltage where the pixels become isolated the number of cross-talk hits drops to zero.

An example of a cross-talk scan at a bias voltage of $46 \mathrm{~V}$ is shown in Figure 6.10(a). The sensor is partially depleted in this specific case. Pixels in the red area still collect charge and thus have a large amount of cross-talk hits, while pixels in the white area are already isolated from each other due to full depletion of this part of the sensor. It is clearly visible that the depleted and undepleted areas have a circular shape. These shapes are created during the production of the sensors. The corresponding silicon wafers are ground rotationally in order to decrease their thickness.

Furthermore, a grid structure is present in every scanned module. Pixels in the grid structure do not represent depleted and undepleted regions correctly, because they collect more charge than expected. Thus, all of the pixels in the grid are excluded from the analysis. Long pixels are the cause for the vertical lines parallel to the pixel row. The horizontal lines parallel to the pixel column are created because the cross-talk scan is performed for several pixels at the same time. In these cases charge is injected in pixels with different column numbers but the same row numbers. This leads to the fact that more than two injection pixels are in close proximity to the readout pixel and the collected charge is larger than initially expected. Ganged and inter-ganged pixels are always excluded from the analysis, because it is not possible to inject charge correctly into these pixels.

Several data points are taken in order to estimate the correct effective depletion voltage. The cross-talk measurement is repeated while the bias voltage is increased step by step up to a maximum of $80 \mathrm{~V}$. The number of pixels with no cross-talk hits is counted for every module as a function of the bias voltage. An example is shown in Figure 6.10(b).

A Gaussian error function is fitted to the data in order to extract the effective depletion voltage:

$$
f(x)=a \cdot \int_{0}^{b \cdot(x-c)} \exp \left(-t^{2}\right) d t+d
$$

where $\mathrm{x}$ corresponds to the bias voltage (also called HV), a,b,c,d are fit parameters, and $\mathrm{f}$ is the number of pixels with zero hits. The effective depletion voltage is defined as $90 \%$ of the

${ }^{1}$ The final results are also summarised in ATL-INDET-INT-2013-001. The internal note is only available for ATLAS members. 
maximum value of the fit function. The number of pixels with no hits is zero for very small values of the bias voltage. The depth of the depleted region grows with increasing bias voltage. A rapid increase of pixel with no hits is recorded as soon as the depleted region approaches the readout side of the sensor.

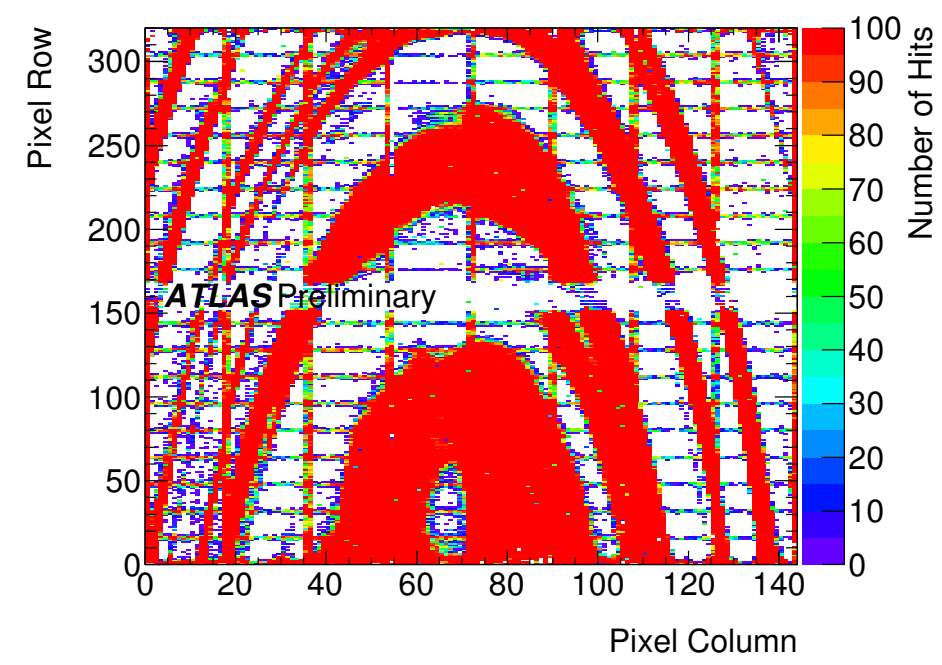

(a)

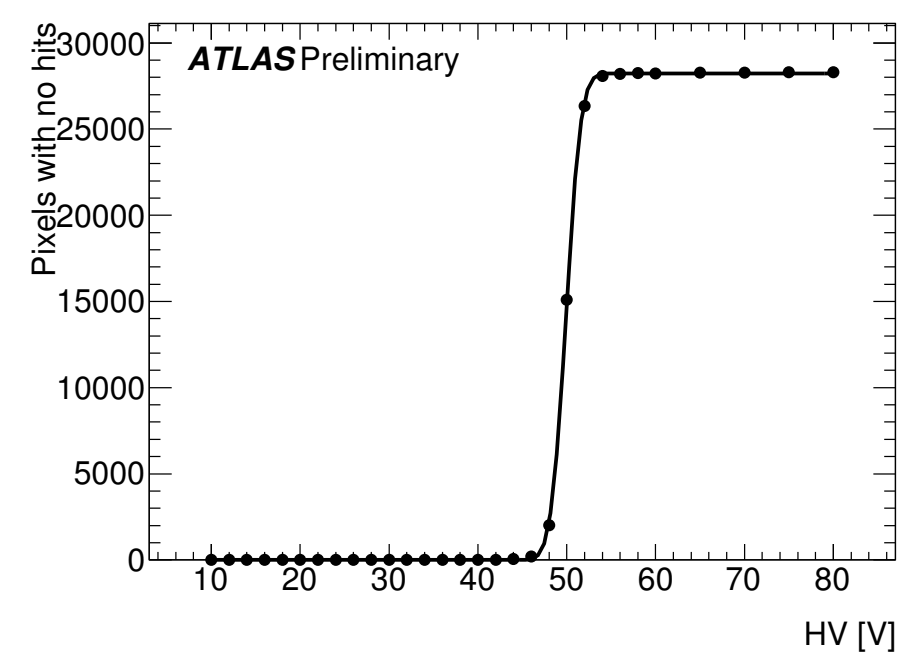

(b)

Figure 6.10.: Example of a cross-talk scan for a partially depleted sensor. The scan was taken at a bias voltage of $46 \mathrm{~V}$ on the 11.05.2011 (a). The number of pixels with no hits as a function of the bias voltage is also shown (b). An error function is fitted to the data in order to extract the effective depletion voltage. Modules L2_B01_S1_A6_M2A (a) and L0_B08_S1_C7_M6C (b) are shown 68. 


\subsubsection{Scans and Module Selection}

Cross-talk scans were taken on a regular basis in order to be able to monitor the development of the effective depletion voltage. In earlier cross-talk measurements only $2 \cdot 60000$ electrons were injected during the scans. However, one side effect of radiation damage is an increasing bulk resistivity. Thus, the injected charge has been increased to $2 \cdot 80000$ electrons at 22.06.2011.

Only scans that were performed after the number of injected electrons were increased are considered in the measurement of the depletion voltage. A summary of these scans is given in Table 6.1. Most of the time only layer 0 has been scanned due to time constraints and because it is the one closest to the interaction point, while three scans have been performed for the whole detector.

\begin{tabular}{|c|c|c|}
\hline $\begin{array}{c}\text { Cross-talk } \\
\text { scan date }\end{array}$ & $\begin{array}{c}\text { Integrated } \\
\text { luminosity }\end{array}$ & $\begin{array}{c}\text { Scanned detector } \\
\text { layers }\end{array}$ \\
\hline \hline 22 June 2011 & $1159 \mathrm{pb}^{-1}$ & Layer 0 \\
8 July 2011 & $1351 \mathrm{pb}^{-1}$ & Layer 0 \\
04 August 2011 & $2047 \mathrm{pb}^{-1}$ & Layer 0 \\
11 August 2011 & $2454 \mathrm{pb}^{-1}$ & Layer 0 \\
15 August 2011 & $2537 \mathrm{pb}^{-1}$ & All layers \\
31 August 2011 & $2768 \mathrm{pb}^{-1}$ & Layer 0 \\
10 November 2011 & $5790 \mathrm{pb}^{-1}$ & Layer 0 \\
27 April 2012 & $6746 \mathrm{pb}^{-1}$ & All layers \\
28 June 2012 & $12544 \mathrm{pb}^{-1}$ & All layers \\
\hline
\end{tabular}

Table 6.1.: List of conducted cross-talk scans. The impact of radiation damage is largest in layer 0 , since it is the innermost detector layer. Therefore, only this layer has been monitored at the beginning.

\section{Module Selection}

It is necessary to exclude several modules from the analysis. Cross-talk scans can fail for many reasons - problems with the readout electronics, high resistivity between pixels etc.

However, every problem changes the shape of the error function. Thus, it is feasible to create a module selection that is solely based on the fit outcome of the error function. The module selection is designed to accept only modules where the shape of the function is within the expected limits, while modules that show a strange behaviour during the cross-talk scans are excluded by the following selection criteria:

- Error function fit has to converge

- Maximum of error function $>20000$ dead pixels

- Minimum of error function $<500$ dead pixels

The number of modules that pass and fail the cuts in layer 0 is given in Table 6.2 , because only this layer is activated in all scans.

The most likely cause of failure is the large resistance between adjacent pixels, even though the charge injection has been increased. It is not possible to increase the charge injection any further. Thus, it is necessary to exclude all modules that still fail the module selection due to 


\begin{tabular}{|c|c|c|c|c|c|}
\hline Date & Deactivated & Not Converged & Minimum & Maximum & Analysed \\
\hline $06 / 2011-04 / 2012$ & 4 & 25 & 12 & 140 & 103 \\
\hline $28 / 06 / 2012$ & 4 & 62 & 17 & 172 & 29 \\
\hline
\end{tabular}

Table 6.2.: Number of modules that pass and do not pass the module selection in the innermost pixel layer.

an increasing high bulk resistance. The impact can be seen in Figure 6.11. The same module is selected that is shown in Figure 6.10(a). It is clearly visible that the resistivity between the pixels has increased.

The bias voltage for the scans is set to $10 \mathrm{~V}$ in Figure 6.11(a) and to $42 \mathrm{~V}$ in Figure 6.11(b). In both cases the number of pixels with no hits is very high. A bias voltage of $42 \mathrm{~V}$ is relatively close to the expected value of the effective depletion voltage and thus a high number of pixels with no hits is reasonable. However, nearly all pixels should have a very larger number of hits if the bias voltage is below the effective depletion voltage, which is clearly the case for a bias voltage of $10 \mathrm{~V}$. This is clearly not the case and thus it is necessary to exclude this module and the ones that show a similar behaviour from the analysis.

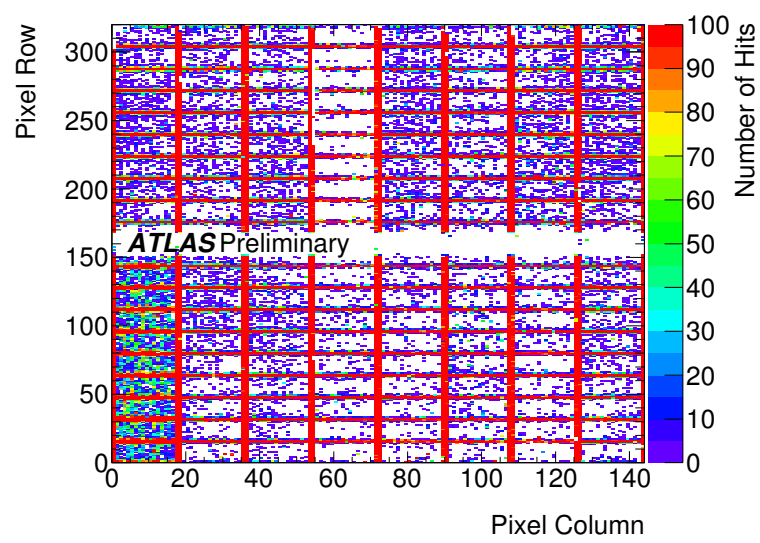

(a)

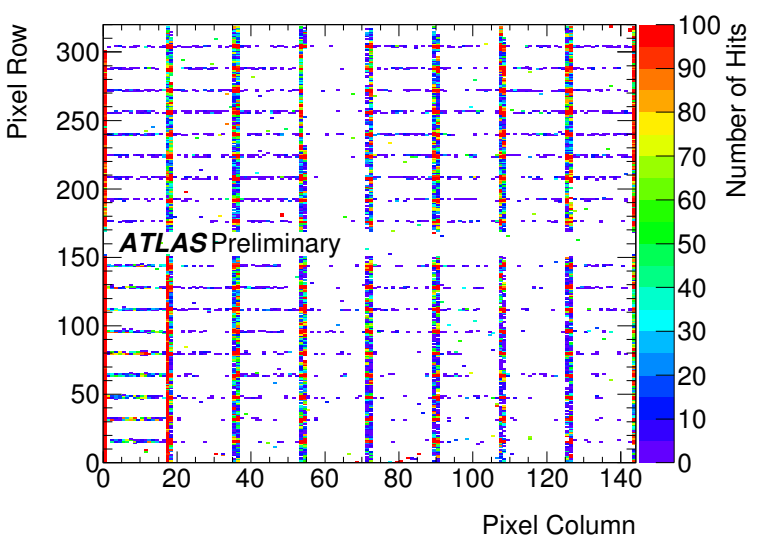

(b)

Figure 6.11.: Plot of the cross-talk scan at the 15.08.2011 for a bias voltage of $10 \mathrm{~V}$ (a) and for a bias voltage of $42 \mathrm{~V}$ (b). Module L2_B01_S1_A6_M2A is shown 68.

\subsubsection{Evolution of the effective Depletion Voltage}

\section{Depletion Voltage Fit and Distribution}

An example of the depletion voltage fit for three different dates is given in Figure 6.12(a). Between 22.06.2011 and 27.04.2012, a decrease in the effective depletion voltage is observed, as is expected before type-inversion. An increase is observed on 08.07.2011. The scan was taken after the cooling had to be stopped between the 29.06.2011 and 03.07.2011. During the cooling stoppage radiation damage induced effects become electrically inactive and thus lead to beneficial annealing. The effect is visible as an increase in the effective depletion voltage.

The doping of the n-type silicon and the creation of crystal defects are statistical processes. In addition, the thickness of the modules varies slightly. Therefore, the effective depletion 
voltage of the whole detector is distributed. Figure 6.12(b) shows the effective depletion voltage distribution of the layer 0 for the same three days as the depletion voltage fit. A similar behaviour is observed for the whole layer as it is for a single module. A decrease in depletion voltage for the whole layer is clearly visible between the first and the last scan, while the whole layer 0 shows signs of beneficial annealing after the cooling stop.

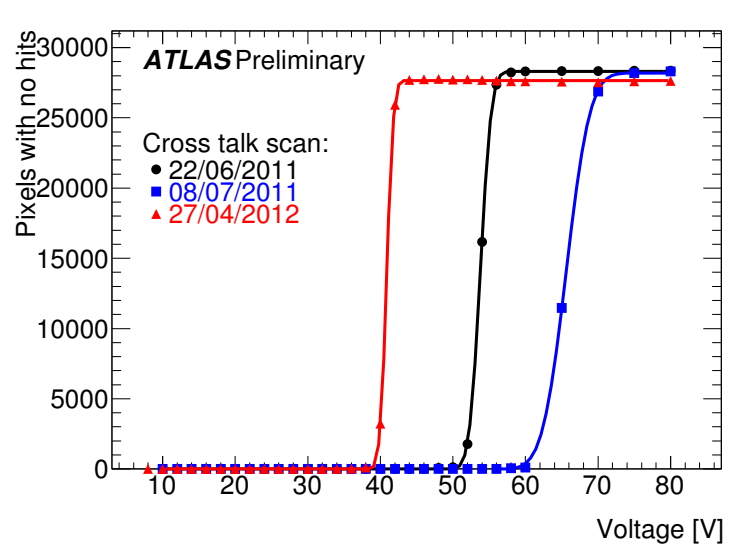

(a)

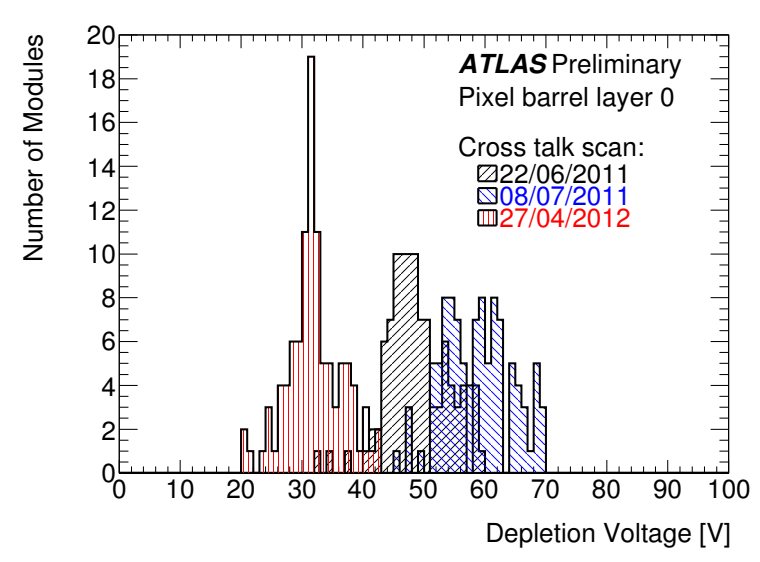

(b)

Figure 6.12.: Results of the depletion voltage scan showing the number of pixels with no hits as a function of the bias voltage. Module L0_B08_S1_C7_M6C is shown (a). Depletion Voltage distributions are shown for all modules in the b-layer that have passed the module selection (b). 103 modules passed the module selection in all three scans 68 .

\section{Evolution of the Depletion Voltage in Layer 0}

The evolution of the effective depletion voltage for single modules in layer 0 is shown in Figure 6.13. Only the modules that have passed the module selection are included. Figure 6.14 shows the mean value of the effective depletion voltage as a function of time, while additional results are shown in Figure A.1. A temperature profile is superimposed at the top of the figure in order to illustrate the temperature rise after the cooling stoppages. It has been extracted from the Detector Control System (DCS) for a single module as an example.

The statistical uncertainty is found to be $\sigma_{\text {stat }} \approx \pm 0.7 \mathrm{~V}$. It is included in Figure 6.14, but it is too small to be visible. A bias in the module selection leads to a systematic uncertainty of $\sigma_{\text {sys }}= \pm 3 \mathrm{~V}$.

A similar behaviour of the effective depletion voltage is observed for the measurement of single modules and for the mean value. In general, the effective depletion voltage decreases with time, as it is expected. Annealing effects during cooling stoppages lead to an increase of the effective depletion voltage. Beneficial annealing has a similar impact on each module, as is shown in Figure 6.13. The increase of the effective depletion voltage is nearly the same for every module in layer 0 , after the temperature has risen.

Many modules in the latest cross-talk scan are close to the expected minimum of the effective depletion voltage and, therefore, are very close to type-inversion or may have started to typeinvert. Thus, they have to be excluded from the analysis, because cross-talk scans are only 
viable as long as the depleted region grows from the backside of the sensor. 103 modules were used in the analysis of the first eight scans, while only 29 modules remain in the last scan, as is clearly visible in Figure 6.13.

Only the remaining modules that still precede type-inversion on the 28.06.2012 are used for the calculation of the last mean value. The effective depletion voltage of the remaining modules is in general larger than the mean value for the whole layer, as is illustrated in Figure6.13. The mean value of the effective depletion in the last scan is therefore larger than it would be for the whole detector layer.

The simulated model prediction of the depletion voltage (dashed line) in Figure 6.14 is based on the delivered luminosity profile, the fluence expectation (FLUKA simulations [69, 70]) and the detector temperature 77,72 . Effects of beneficial annealing during the cooling stoppages are included in the model, their impact is larger in data than it is in the model prediction. Comparing the second and the third scan, it is visible that the decrease in the effective depletion voltage after annealing is slightly faster than the long-term decrease. Overall, the modelled depletion voltage decreased faster than the measured effective depletion voltage, most likely caused by the underestimation of beneficial annealing in the model prediction.

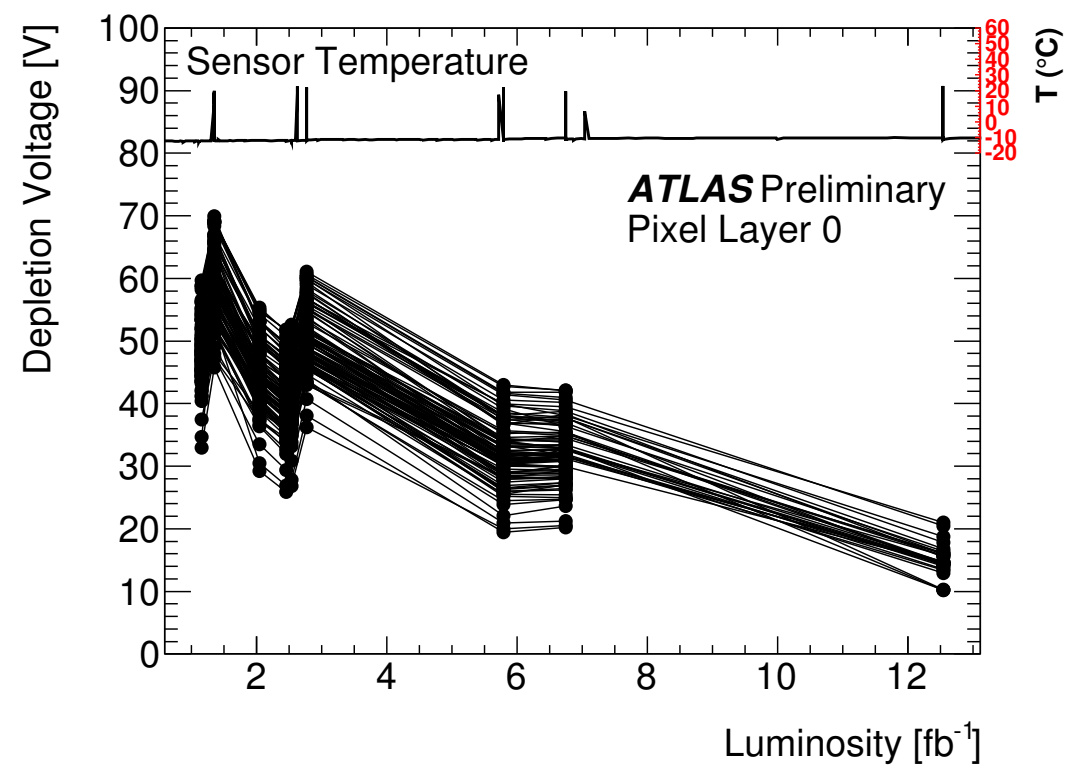

Figure 6.13.: Effective depletion voltage as a function of the delivered luminosity. Each point shows the effective depletion voltage for a single module in layer 0. A temperature profile for a single module is added at the top in order to illustrate the temperature rise after the cooling stoppages [68].

\section{Dependence on Module Position}

Figure 6.15 shows the change in the effective depletion voltage between the first and second to last cross-talk scan as a function of the module position in $z$. Only modules in layer 0 are analysed. The second to last scan is chosen, because the number of modules that pass the module selection is equal for both of these scans.

The position on the stave parallel to the beam-line is called module position in $z$. The 


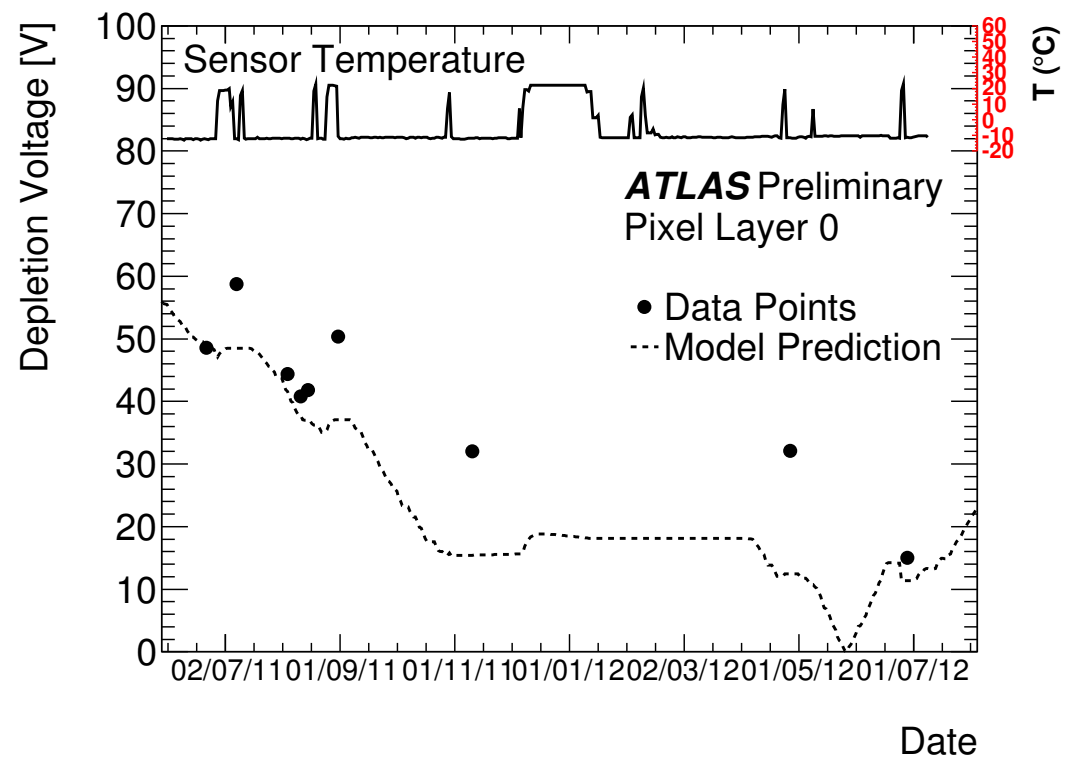

Figure 6.14.: The mean value of the effective depletion voltage in layer 0 as a function of the date is shown. The model prediction for layer 0 is superimposed as a dashed line and an example of the module temperature is added at the top. The statistical error is included, but it is too small to be seen in this Figure and the systematic uncertainty is found to be $\sigma_{\text {sys }}= \pm 3 \mathrm{~V}$ [68].

interaction point is at the origin and the higher the number the further away is the module from the interaction point. Modules that are located on the A side have positive module positions, while modules with negative module positions are located on the $\mathrm{C}$ side of the detector.

The particle fluence is larger at the center of the detector, and thus it is expected that the impact of radiation damage is more severe for modules close to the interaction point. Modules in the center clearly show a larger change in the effective depletion voltage than the outer modules. The change is $\sim 3 \mathrm{~V}$ larger in the centre.

Furthermore, a second illustration of the larger impact of radiation damage in the center of the detector is shown in Figure 6.16(a). The change in the depletion voltage is shown as a function of the module and stave number. This time the change is calculated by taking the difference of the second scan and the last scan. Only staves in layer 0 are shown and the module number is identical to the module position in $z$. It is expected that the modules in the centre of the detector fail in the cross-talk scan earlier than the outer modules, due to the very high particle fluence at the interaction point. All of the 29 modules that remain active in the last scan have a module position of two or larger, while all inner modules are excluded from the analysis. This is a strong indication that the modules in the centre started to undergo type-inversion earlier.

With the help of a FLUKA simulation it is possible to calculate the received $1 \mathrm{MeV}$ neutron equivalent fluence for each module in the layer 0. Figure 6.16(b) shows the effective depletion voltage as a function of the $1 \mathrm{MeV}$ neutron equivalent fluence. The modules are divided into inner and outer modules. Inner modules have a module position in $\mathrm{z}$ that is smaller than three. The collected fluence is in general larger for inner modules, as expected. 


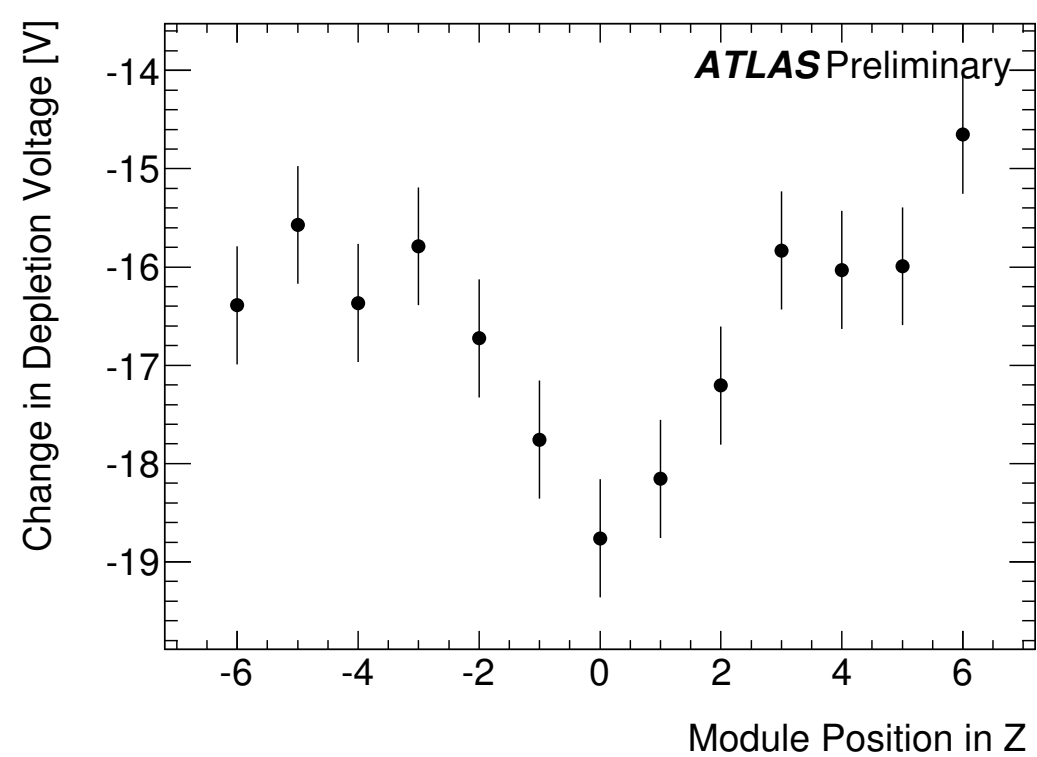

Figure 6.15.: Depletion Voltage difference between 22.06 .2011 and 17.04 .2012 as a function of the module position. The mean value is calculated by summing up all modules in layer 0 with the same stave position. The larger the module position in $z$ the further away is the module from the interaction point. A high fluence at the centre leads to a faster decrease in the effective depletion voltage in the corresponding modules [68].

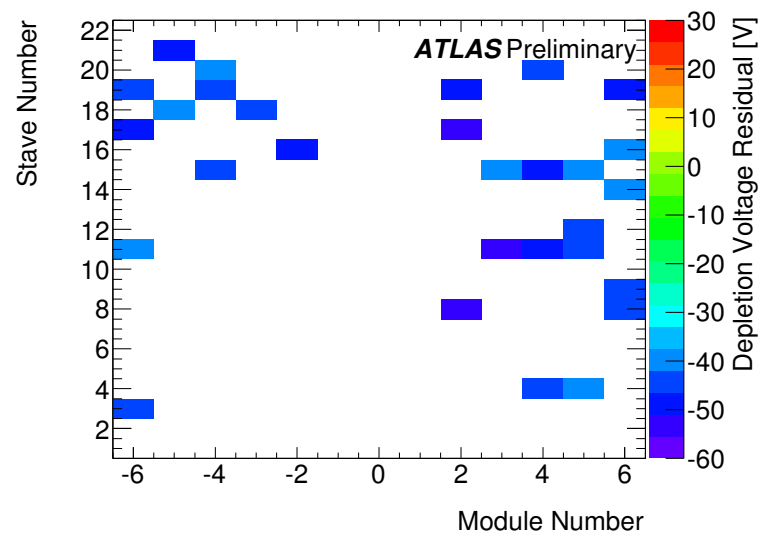

(a)

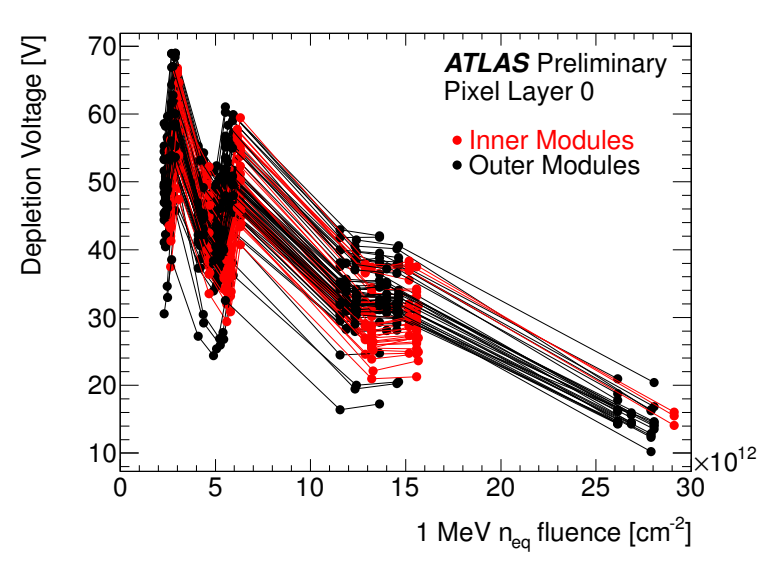

(b)

Figure 6.16.: Depletion voltage residual shown for the 29 modules that still precede typeinversion. The depletion voltage residual is the difference of the effective depletion voltage between the 08.07.2011 and 28.06.2012 (a). Depletion Voltage as a function of the neutron equivalent fluence (b) 68. 


\section{Depletion Voltage Measurements in Layer 1 and 2}

Figure 6.17(a) and 6.17(b) show the results of the cross-talk scans for layer 1 and 2 as a function of time. Results as a function of luminosity are shown in A.2. Due to time constraints, only three scans have been taken for the outer layers. The dashed line represents the model prediction and the sensor temperature is superimposed at the top of the figure.

The evolution of the effective depletion voltage for layer 1 and 2 is very similar to that of layer 0. A general trend of reduction in the effective depletion voltage is visible, while signs of beneficial annealing are observed after the cooling stoppages. The decrease in the effective depletion voltage is fastest for layer 0 and slowest for layer 2, as expected. Both outer layers do not show signs of type-inversion in summer 2012, while strong indications for type-inversion are visible in the cross-talk scan of layer 0 .

The impact of annealing in the model prediction is similar for all three layers. This is not the case in data - if Figures 6.14, 6.17(a) and 6.17(b) are compared, it is visible that the impact of beneficial annealing becomes larger the closer the detector layer is located to the beam line. Therefore, the discrepancy between collision data results and the model prediction is smallest for Layer 2.

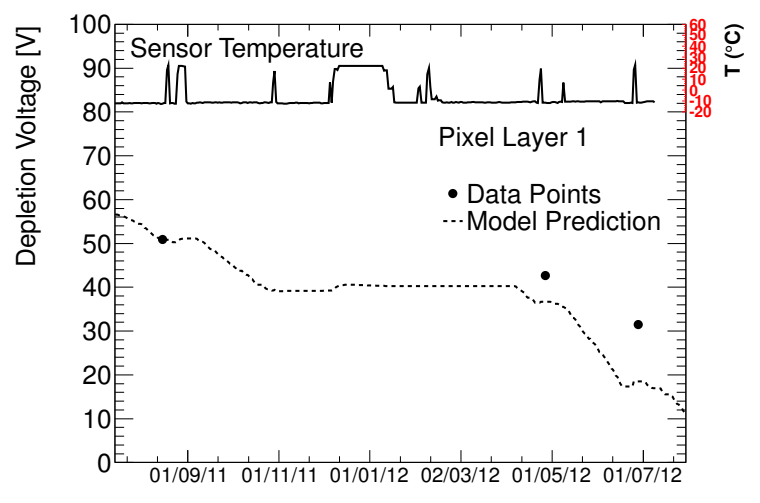

Date

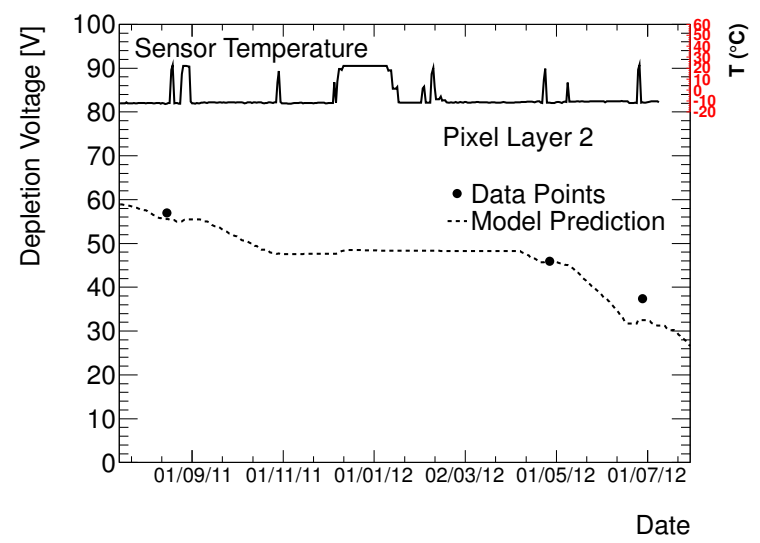

(b)

Figure 6.17.: Evolution of the effective depletion voltage as a function of time shown for layer 1 (a) and layer 2 (b). The sensor temperature is added at the top and the model prediction is superimposed as a dashed line.

\section{Summary}

The evolution of the effective depletion voltage before type-inversion is routinely monitored. A general trend of reduction is measured in all three layers of the ATLAS pixel detector. Beneficial annealing is observed after cooling stoppages. A similar behaviour is observed for the evolution of the mean value and for single module measurements. The model prediction shows a similar behaviour. However, The impact of beneficial annealing is larger in data than it is in the simulated model prediction.

The results for all layers are summarised as a function of the $1 \mathrm{MeV}$ neutron equivalent fluence in Figure 6.18. The expected fluence with a dependence on the radius is calculated using FLUKA Monte Carlo simulations. 
The cross-talk scans show that the effective depletion voltage in the innermost layer is close to its minimum in summer 2012. This indicates that layer 0 underwent type-inversion in summer 2012, this observation is consistent with the model prediction. Layer 1 and 2 do not show any signs of type-inversion in the last scan. However, layer 1 is expected to undergo type-inversion in fall 2012, while the start of type-inversion in layer 2 is not expected before the first long shutdown of the LHC between 2013 and 2015.

New methods for monitoring the impact of radiation damage have been developed, in order to confirm that type-inversion has occurred. In addition, the new measurements ensure that a continuous monitoring of radiation damage after type-inversion is possible, since cross-talk scans can only be used for sensors preceding type-inversion. Track based depletion depth measurements replace the cross-talk scan based depletion voltage measurements. Measurements of the depletion depth are explained in the following.

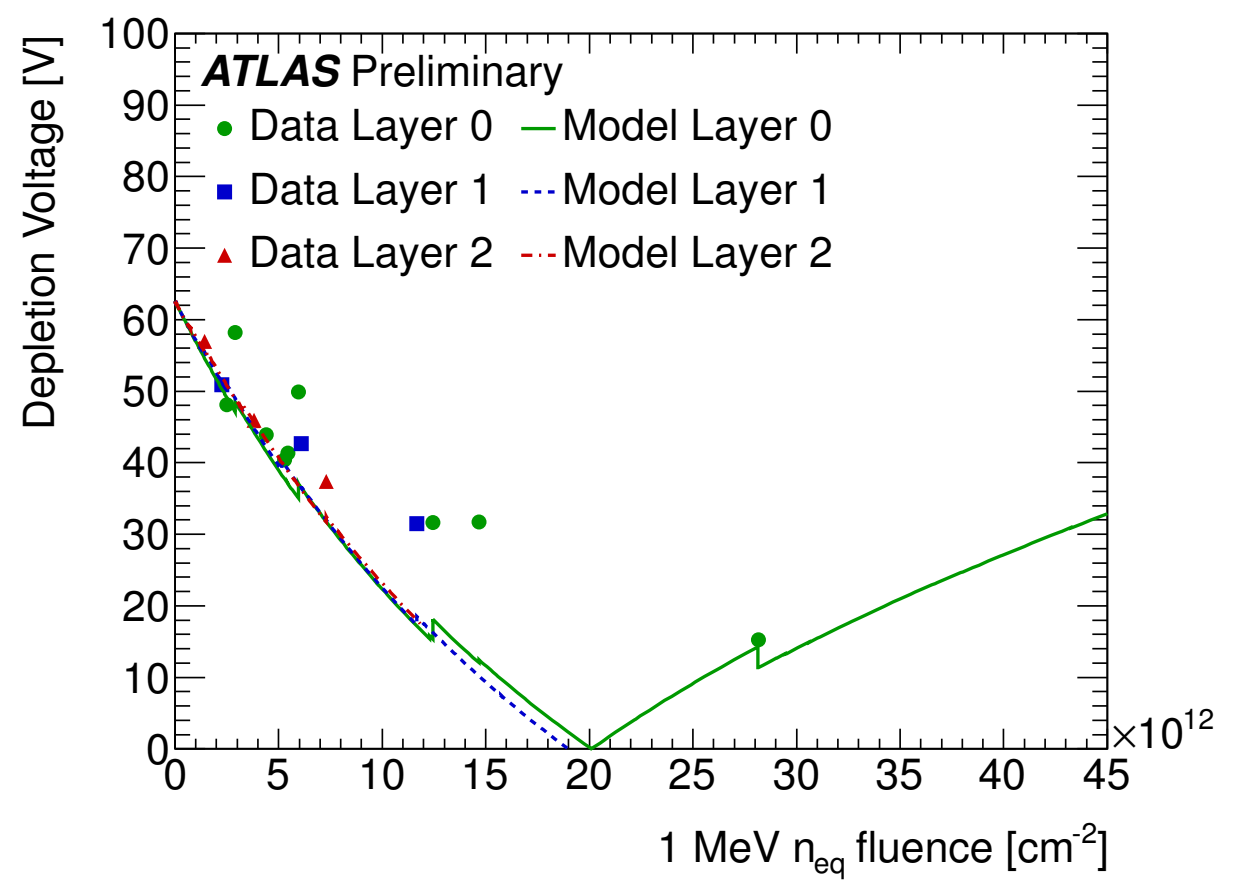

Figure 6.18.: Mean value of the effective depletion voltage as a function of the $1 \mathrm{MeV}$ neutron equivalent fluence shown for all three detector layers. Model predictions for each layer are superimposed. The calculation of the $1 \mathrm{MeV}$ neutron equivalent fluence is based on FLUKA simulations 68 .

\subsection{Track Position based Depletion Depth Measurement}

\subsubsection{Measurement Method}

The charge collection in the pixel detector is closely related to the depletion depth in the sensor. A fully depleted sensor and a partially depleted sensor are shown Figure 6.19. The depleted region is represented by the white area, while the undepleted region is shown in grey. A particle passing through the sensor is illustrated as a black line. Pixels that have collected enough charge to reach the discriminator threshold are white, while black pixels do not collect enough charge. 
Charge is collected as long as the particle passes through the active region in the sensor and the corresponding pixels show a hit. No charge is collected in the undepleted region, because the created electron-hole pairs recombine again on a very short time-scale. Thus, the total collected charge is proportional to the size of the depletion depth. Furthermore, the cluster-size is a function of the depletion depth as is demonstrated in Figure 6.19(a) and 6.19(b). These relationships allow the measurement of the depletion depth using reconstructed particle tracks.

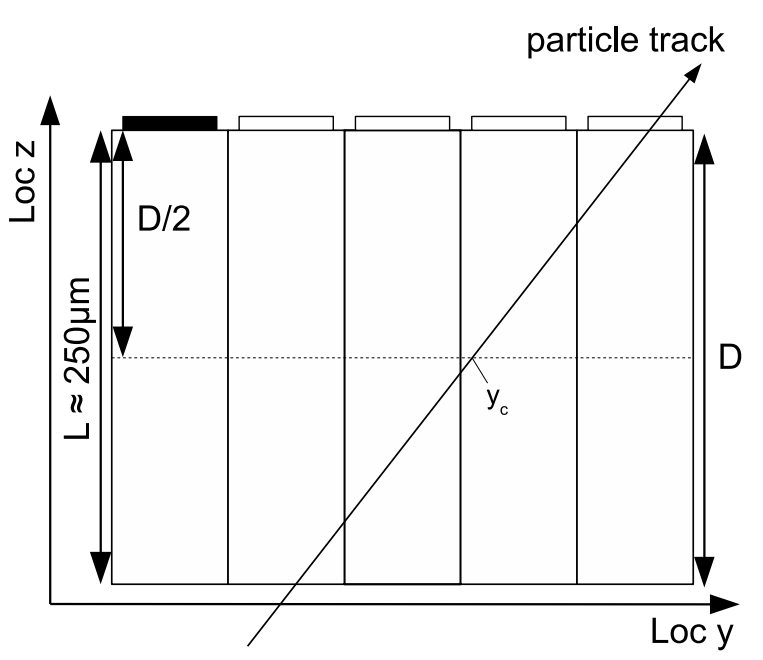

(a)

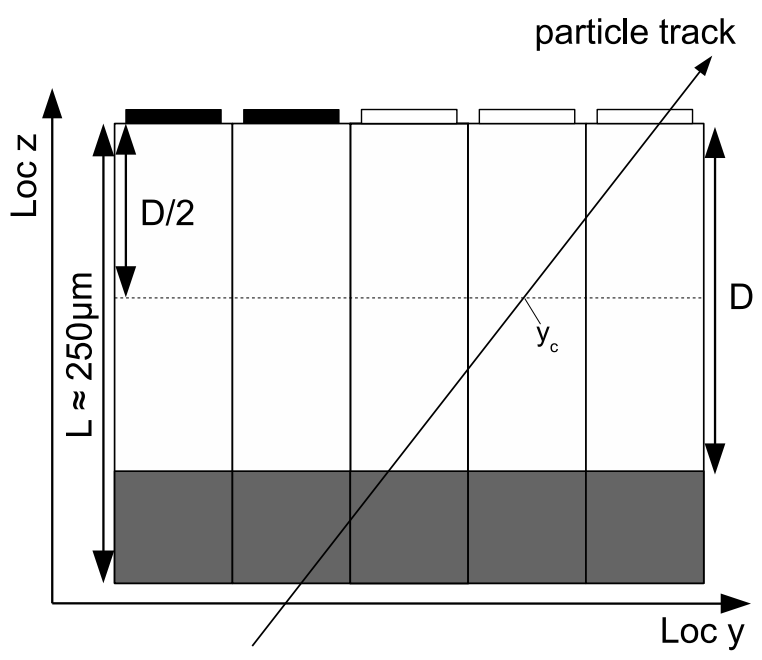

(b)

Figure 6.19.: Illustration of the pixel sensor fully depleted (a) and partially depleted (b). A two dimensional projection in long pixel direction is shown (not to scale).

It is assumed that the collected charge is only a function of the depletion depth. This simplification is only valid for non and slightly irradiated sensors. In highly irradiated sensor additional effects of radiation damage such as trapping have to be considered. More details are given in Subsection 6.5.2.

The first track based measurements were developed to estimate the depletion depth of irradiated modules in early test-beam data [73], [38]. The conditions in the test-beam environment are different to the conditions in the ATLAS detector. Thus, it was necessary to adapt the track based depletion depth measurements after the installation of the pixel detector. First measurements of the depletion depth in the ATLAS pixel detector were performed in 2008 using cosmic ray data [74. Cosmic rays travel mostly from the top of the detector to the bottom, leading to a large range of incidence angles in the local $R-\varphi$ plane of the pixel module.

After the start of the LHC the situation changed again, the angular distribution for collision data differs from the cosmic ray distribution and since collision data are taken with the magnetic field turned on, Lorentz Angle effects have to be considered. In addition, the older methods rely partially on Monte Carlo data, in order to take threshold effects into account.

The new fully data driven track position based depletion depth measurement has the advantage that it can be used on a wide angle range, since only a minimum cluster size of 2 is needed in order to estimate the depletion depth using this method. First results have been presented in the course of a conference in summer 2012 [67].

The tracks are always projected onto one of the planes of the pixel modules. Two projections can be used, the one in the long pixel direction (local y-z plane) and the one in the short pixel 
direction (local $R-\varphi$ plane). In general the measurement works for both projections. In earlier measurements 74$]$ the projection in the short pixel direction has been used.

The pixel size in the long direction is larger than the sensor thickness. The track position method described in this section is very precise if the pixel width is smaller than the sensor thickness, however, collision data are taken with the magnetic field turned on. The impact of the charge drift caused by the Lorentz force is large in the short pixel direction, while it is negligible in the long pixel direction. In addition, the range of incidence angles in the $R-\varphi$ plane is narrow (range $\sim 0.2-0.5[\mathrm{rad}]$ ). To avoid these issues the projection has been changed. All particle tracks are projected along the long pixel direction, because the magnetic field lines are parallel to the local y-axis.

A scheme of the pixel sensor in the long pixel direction is shown in figure 6.20, where $L$ is the assumed thickness of the pixel sensor and $D$ is the depletion depth of the pixel sensor. In principle only two variables are needed to calculate the depletion depth. These values are the track position at the readout side on the surface of the sensor $y_{0}$ and the track incidence angle $\theta$. Track incidence angles are calculated during the reconstruction phase with very high precision. The track position $y_{0}$, however is unknown, while the position of the track in the centre of the active region $y_{c}$ is known. Thus, the track position can only be approximated as $y_{0}^{\prime}$. It is possible to correct the approximation and calculate the depletion depth, as is explained in the following.

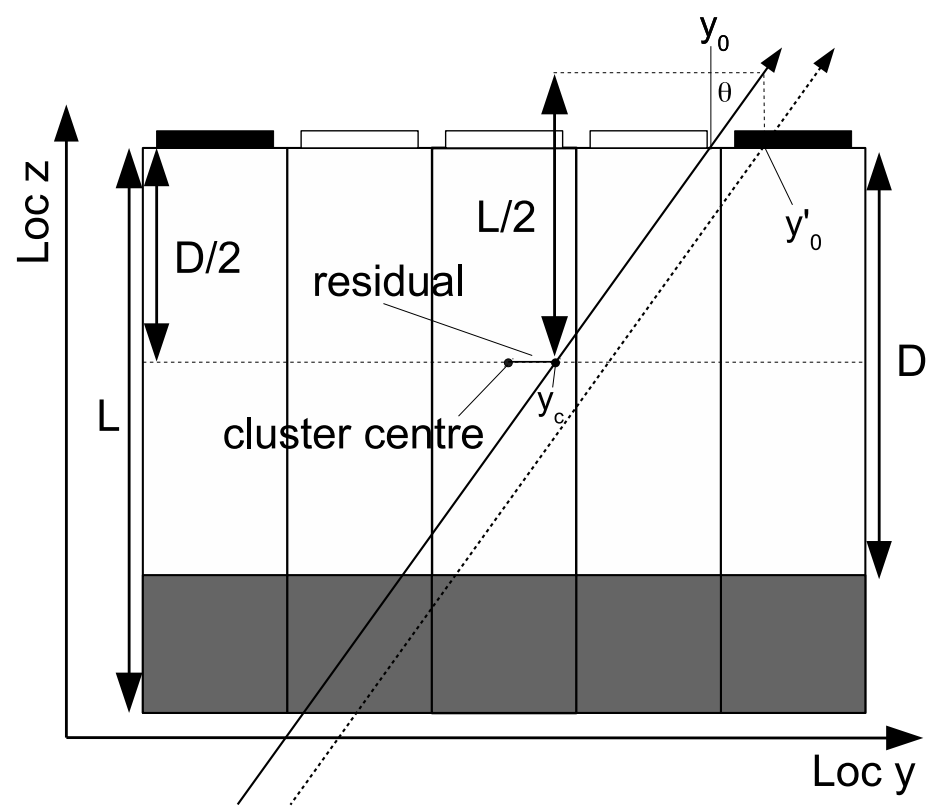

Figure 6.20.: A particle passing through the pixel sensor. The track position on the surface of the sensor $y_{0}$ is needed for the calculation of the depletion depth. The known value is the position of the track in the centre of the active region $y_{c}$ (not to scale).

The trigonometric function for the calculation of the track position on the surface $y_{0}$ is defined as:

$$
y_{0}=y_{c}+\frac{D}{2} \tan \theta .
$$

The extrapolated cluster position $y_{c}$ is calculated in the centre of the depletion depth (local $z$ 
direction). Hence, the distance in local z between $y_{c}$ and $y_{0}$ is $\frac{D}{2}$. Since the depletion depth is unknown, $D$ is replaced by the sensor thickness $L$ leading to the following approximation:

$$
y_{0}^{\prime}=y_{c}+\frac{L}{2} \tan \theta .
$$

The result of this replacement is a systematic uncertainty on the track position $y_{0}$ :

$$
y_{0}^{\prime}-y_{0}=\frac{L-D}{2} \tan \theta .
$$

The thickness $L$ is chosen to be $250 \mu \mathrm{m}$. The difference $y_{0}^{\prime}-y_{0}$ can be measured by using the pixel charge. Therefore, it is possible to retrieve the depletion depth:

$$
D=L-2 \frac{y_{0}^{\prime}-y_{0}}{\tan \theta} .
$$

However, a systematic misalignment $\Delta \tilde{y}$ also affects the calculation of the entry point. Thus, the systematic shift $y_{0}^{\prime}-y_{0}$ of the entry point is calculated to be:

$$
y_{0}^{\prime}-y_{0}=\frac{L-D}{2} \tan \theta+\Delta \tilde{y} .
$$

Leading to

$$
D=L-2 \frac{y_{0}^{\prime}-y_{0}}{\tan \theta}+\frac{2 \Delta \tilde{y}}{\tan \theta} .
$$

Two variables in 6.18 remain unknown and need to be estimated in order to be able to measure the depletion depth $D$. The measurement of $y_{0}^{\prime}-y_{0}$ is shown in Figure 6.21. The mean value of the deposited charge is proportional to the travel distance of a particle in the active region of the sensor.

If the incidence angle is fixed, then the total distance is constant, while the distance in each pixel depends on the track position $y_{0}$ of the particle, as is illustrated in Figure 6.21(a). The travelled distance in the last pixel increases between track 1 and track 2 and so does the collected charge. However, the maximum distance is reached as soon as the surface point is equal to the pixel edge (track 2) and the collected charge in track 2 and 3 are equal as long as the threshold of the neighbouring pixel has not been reached. The difference between the real knee position and the edge of the pixel is equal to the difference between the track position and the approximation $y_{0}^{\prime}-y_{0}$.

The knee position is estimated with the following fit function:

$$
q(y)=a(y-b) \theta(y-b)+c,
$$

where $\theta(y-b)$ is the step function and a,b,c are fit parameters. The effect of varying incidence angles is handled by grouping the incoming particle tracks into different slices, depending on the corresponding angle. Tracks with different angles are filled into different charge histograms. One of these histograms is shown as an example in Figure 6.21(b).

A second measurement is used to estimate the effect of the systematic shift $\Delta \tilde{y}$. The essential values needed for this measurement are shown in Figure 6.22. The track depth is defined as the distance between the track and the surface of the sensor on the readout side (see Figure 6.22(a)). It is measured at the centre of each active pixel in the cluster. To calculate this value it is necessary to know the cluster size, the incidence angle and the track position of the particle on the surface $y_{0}$. 


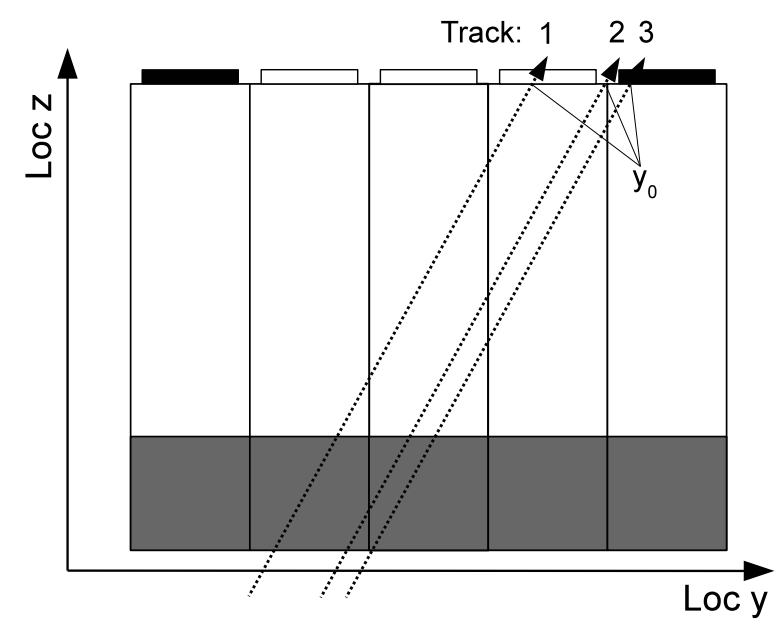

(a)

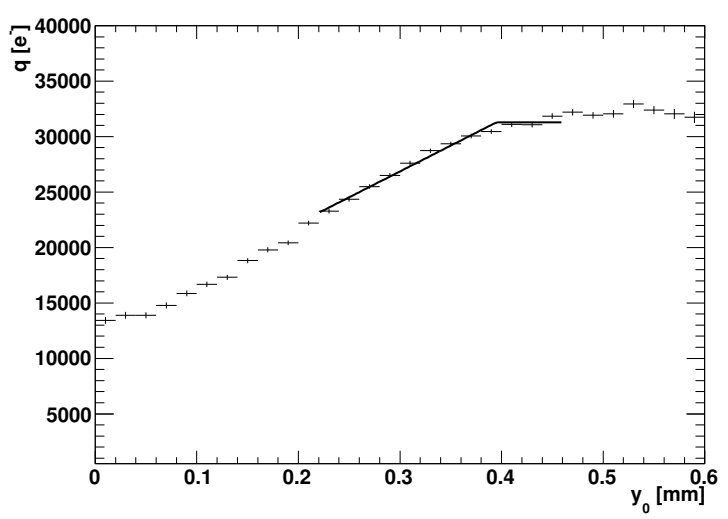

(b)

Figure 6.21.: Schematic view of the sensor. Three tracks with different track positions are shown (a). Fit of the knee position is shown for tracks with incidence angles between 1.2 and 1.24 radians (b).

Track depth values are closely related to the depletion depth of the sensor. Figure 6.22(b) shows an example of the maximum and the minimum track depth in a cluster. The maximum track depth for a positive angle of incidence is measured when the first pixel in the cluster is just above threshold and for a negative track depth when the last pixel is just above threshold. The difference between the maximum track depth and the depletion depth $c$ is equal to the minimum track depth. Therefore, it would be possible to calculate the depletion depth directly, if the track position $y_{0}$ were known. This is not the case as mentioned before.

The approximation of the track position $y_{0}^{\prime}$ introduces a systematic shift $A=\frac{L}{2}-\frac{D}{2}$ in the minimum and maximum track depth, as is illustrated in Figure 6.23. Due to this shift, it is not possible to calculate the depletion depth directly using the track depth. However it is possible to use the track depth as a measurement of misalignment since the depletion depth can be calculated using the direct relationship between the real and the approximated track position $y_{0}^{\prime}-y_{0}$.

The shifted entry point $y_{0}^{\prime}$ is used to obtain the approximated track depth $t d^{\prime}$ :

$$
t d^{\prime}=\frac{y_{0}^{\prime}-y_{i}}{\tan \theta} .
$$

To estimate the discrepancy between the real track depth $t d$ and the approximated track depth $t d^{\prime}$, it is necessary to replace the calculated track position $y_{0}^{\prime}$ in equation 6.20 with the real track position. The outcome is:

$$
t d^{\prime}=t d+\frac{L-D}{2}+\frac{\Delta \tilde{y}}{\tan \theta}
$$

where

$$
t d=\frac{y_{0}-y_{i}}{\tan \theta} .
$$



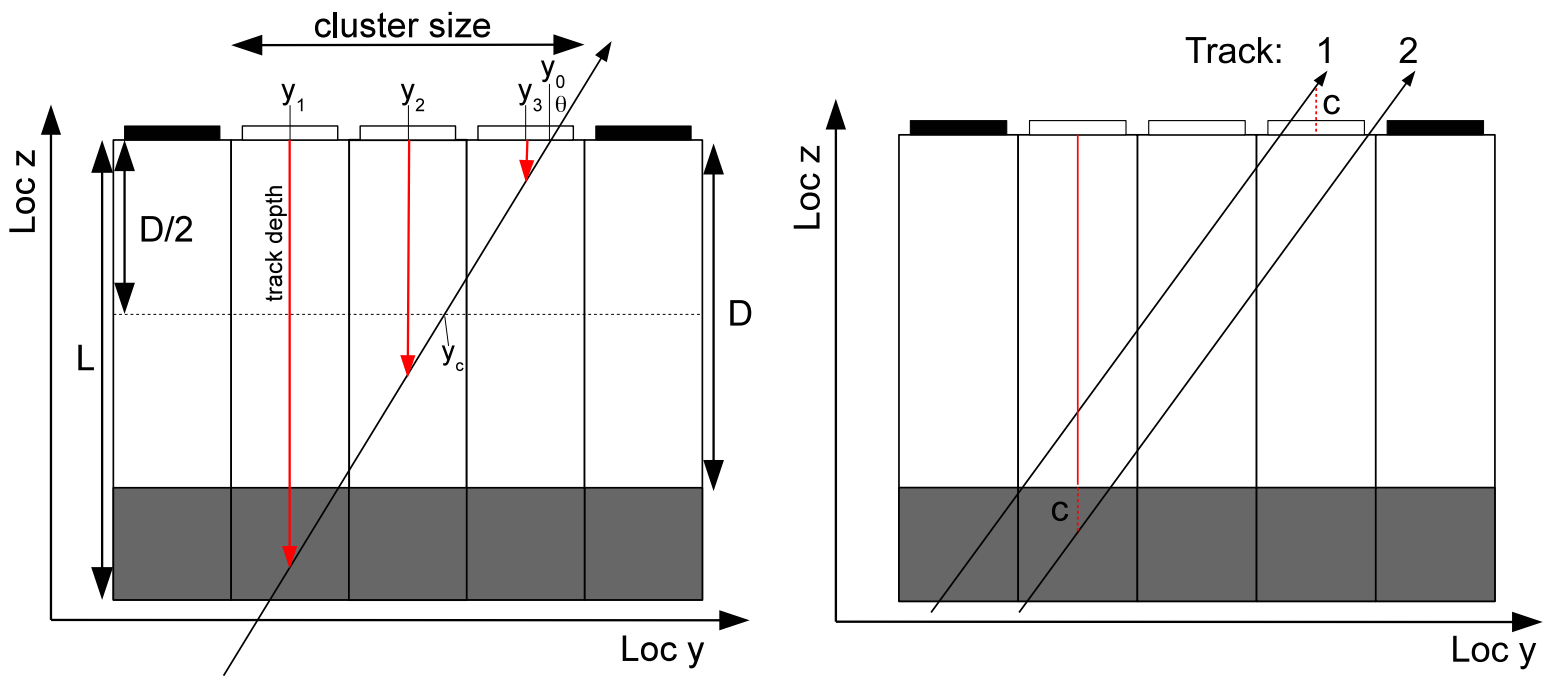

Figure 6.22.: Schematic view of the sensor. The track depth is defined as the distance between the track in the centre of each pixel and the readout-side of the sensor (a). $c$ is the difference between the maximum track depth and the depletion depth (b).
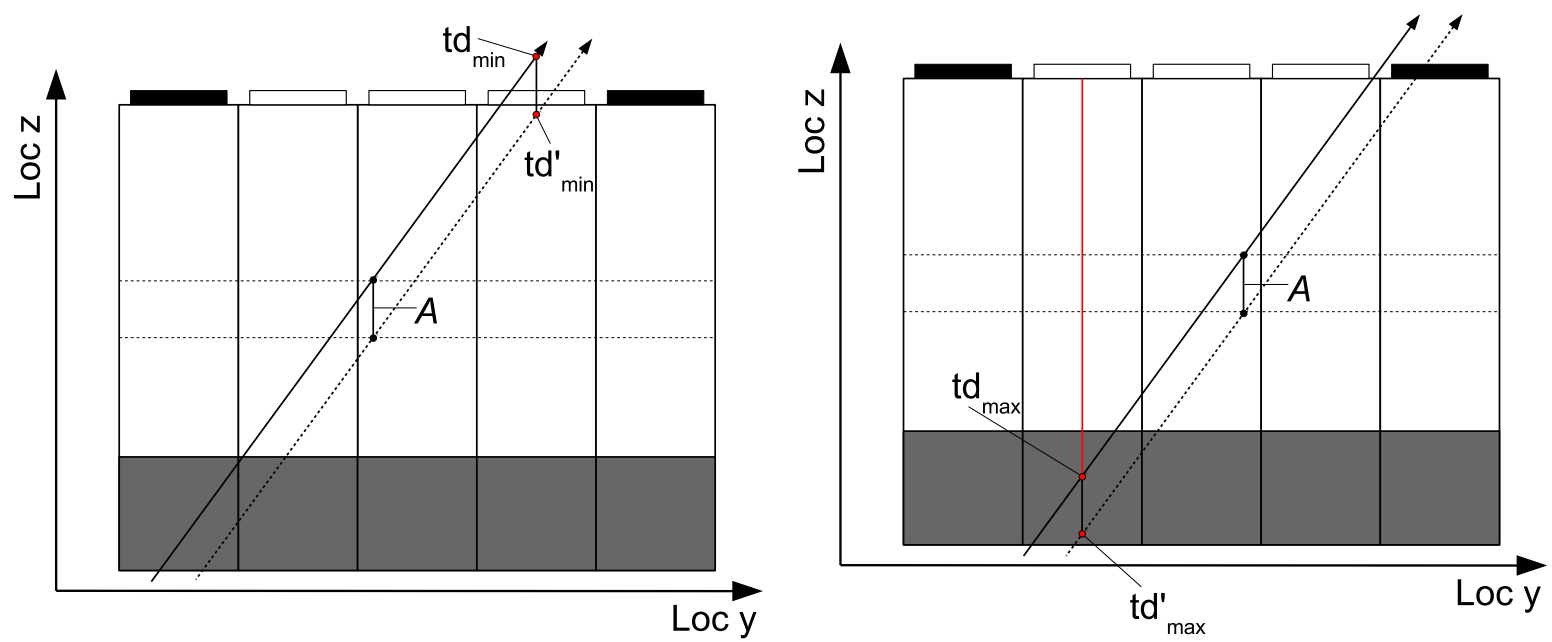

Figure 6.23.: Overestimation of the minimum track depth (a) and maximum track depth (b). Both track depths are overestimated by the same amount $A$ due to the approximation of the entry point. 
The approximation of the maximum and the minimum track depth for a fixed angle is used to estimate the shift. They are added:

$$
t d_{\max }^{\prime}+t d_{\min }^{\prime}=t d_{\max }+t d_{\min }+L-D+\frac{2 \Delta \tilde{y}}{\tan \theta} .
$$

The sum of the real maximum $t d_{\max }$ and the minimum track depth is equal to the depletion depth $D=t d_{\max }+t d_{\min }$. This means:

$$
t d_{\max }^{\prime}+t d_{\min }^{\prime}=L+\frac{2 \Delta \tilde{y}}{\tan \theta} .
$$

The minimum and maximum track depth values are a function of the discriminator threshold and the incidence angle. Effects of varying incidence angles are handled the same way as they are handled for the knee position fit. Tracks with different angles are filled into different track depth histograms. One of these histograms is shown as an example in Figure 6.24. Two error functions are fitted to the track depth histogram to estimate the approximated maximum and minimum track depth:

$$
f(x)=a \cdot \int_{b \cdot\left(x-c_{1}\right)}^{\infty} \exp \left(-t^{2}\right) d t
$$

if $x<\frac{c_{1}+c_{2}}{2}$ and

$$
f(x)=a \cdot \int_{-b \cdot\left(x-c_{2}\right)}^{\infty} \exp \left(-t^{2}\right) d t
$$

if $x>\frac{c_{1}+c_{2}}{2}$. The fit parameters are $a, b, c_{1}$ and $c_{2}$. The inflection points $c_{1}$ and $c_{2}$ are equal to the approximated track depth values for a fixed angle of incidence.

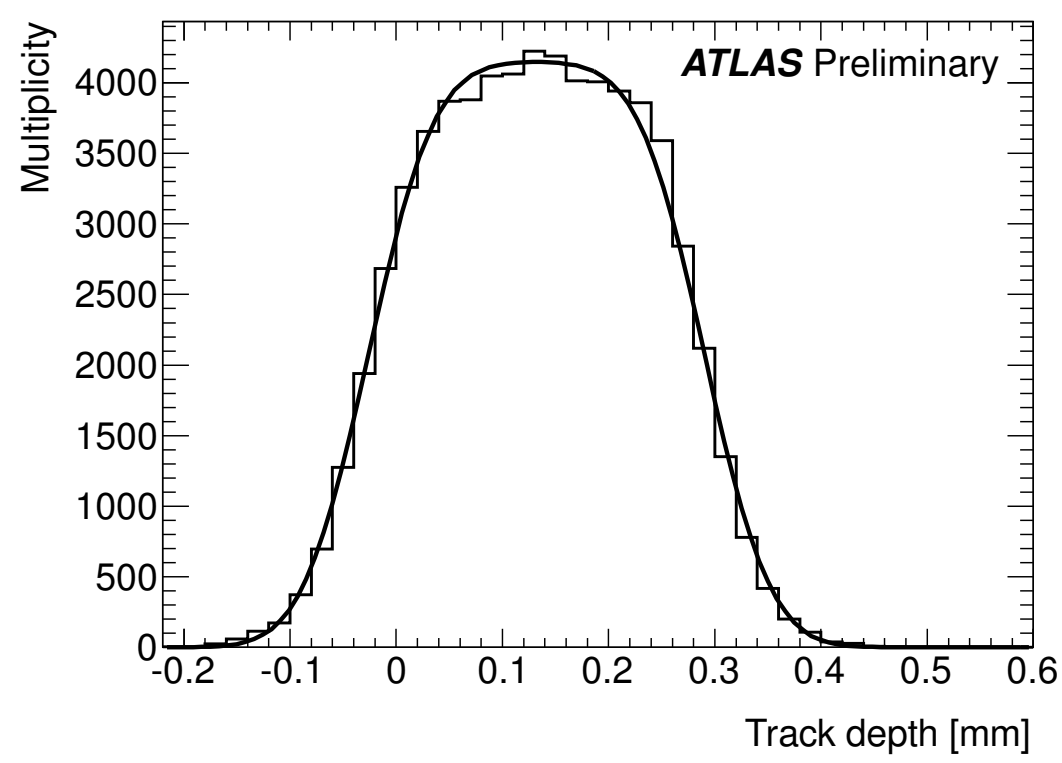

Figure 6.24.: Track depth distribution and error function fit. The minimum and maximum track depth values are defined by the inflection point of the error function fit [68]. 
Finally, Equation 6.24 and 6.18 are combined to create the final formula for the estimation of the depletion depth as a function of the approximated track depth $t d^{\prime}$, the difference in track position $y_{0}^{\prime}-y_{0}$ and the incidence angle $\theta$ :

$$
D=t d_{\max }^{\prime}(\theta)+t d_{\min }^{\prime}(\theta)-2 \frac{y_{0}^{\prime}(\theta)-y_{0}(\theta)}{\tan \theta} .
$$

\subsubsection{Sources of Systematic Uncertainties}

The systematic uncertainty in the charge calibration is overall $\sigma \approx 400$ electrons, while the standard deviation can be as large as 1000 electrons. In addition, the mean value of the uncertainty is a function of the collected charge in the pixel and it tends to be bigger if the collected charge is larger.

The knee position is not shifted as long as the miscalculation of the charge is constant. However, the dependence of the systematic uncertainty in the charge calibration on the collected charge leads to a shift in the knee position (see Figure 6.21(b) and thus, finally to a systematic uncertainty of the depletion depth. The total systematic uncertainty is found to be $\sigma_{\text {sys }} \approx 10 \mu \mathrm{m}$. It is retrieved by varying the starting parameters of the knee position fit.

In the future, additional effects of radiation damage have to be taken into account. Trapping decreases the amount of collected charge in the whole sensor and therefore the likelihood that a pixel reaches its threshold. Earlier measurements of the depletion depth relied mainly on the relationship between the cluster size and the depletion depth and are thus very susceptible to trapping effects. The result of the measurement in highly irradiated sensors is therefore an effective distance of charge collection.

The new method relies mainly on the relationship between the collected charge in each pixel and the depletion depth and is therefore less susceptible to any uniform decrease in charge collection. Trapping centres are expected to be distributed homogeneously in the whole sensor and thus charge carriers are lost in the sensor. However, the Shockley-Ramo theorem 24] states that free charge carriers need to be close to the pixel implants in order to induce sufficient charge in the readout electronics. Particles created closer to the backside of the sensor, thus, need to travel through the sensor bulk towards the pixel side before they can generate a signal. The larger distance that needs to be covered in this case leads to an increased trapping probability for electrons created deep inside the sensor bulk. Therefore, in heavily irradiated sensors the charge collection decreases with increasing track depth. A study of the impact has been conducted using ATLAS pixel detector test beam data [75]. The change in charge collection will most likely lead to a smearing of the charge distribution in Figure 6.25 and thus might also cause a displacement of the knee position.

Furthermore, radiation damage has an impact on the electric field inside the sensor. The field configuration differs from the linearly decreasing field as is observed in un-irradiated $\mathrm{p}-\mathrm{n}$ junctions operated in reverse bias. A promising model assumes a double junction configuration [76]. The complicated field configuration makes it more and more difficult to define a strict threshold between the depleted and the undepleted area with increasing fluence. Due to these two effects the measurement of the depletion depth in heavily irradiated sensors also becomes a measurement of an effective depth of charge collection. However, measurements of the effective charge collection depth are also extremely useful, since this value is a very good indicator of the impact of radiation damage and sensor performance.

\footnotetext{
${ }^{2}$ The values are taken from ATL-INDET-INT-2011-001. The internal note is only available for ATLAS members.
} 


\subsubsection{Data Samples and Track Selections}

In the presented analysis, the collision data from two runs, with a centre-of-mass energy of $7 \mathrm{TeV}$, are used. Table 6.3 shows a summary of the used data runs.

\begin{tabular}{|c|c|}
\hline Run & Date \\
\hline 182516 & 24.05 .2011 \\
187815 & 22.08 .2011 \\
\hline
\end{tabular}

Table 6.3.: Runs analysed with the track position based depletion depth measurements.

Tracks have to pass the basic track quality cuts as well as additional cuts connected with cluster characteristics.

- Momentum cut. Tracks need a transverse momentum ${ }^{3}$ of $p_{T}>2 \mathrm{GeV}$.

- Isolation cut. There is no other cluster on the same module within $2 \mathrm{~mm}$.

- No ganged pixels and no long pixels. Ganged pixels and long pixels between the FE chips are excluded.

- First rows and columns. In order to avoid edge effects the cluster should not contain any cells belonging to the first or the last two rows and columns of pixels.

- Extrapolated position. The extrapolated position of the track $y_{c}$ must be located between the first and the last hit pixel.

- Minimum pixel barrel hits. Tracks with less than 3 hits in the Pixel Barrel are excluded.

- Cluster size. The cluster size in the local $\mathrm{x}$ direction has to be smaller than 4 pixels and the cluster size in the local y direction has to be smaller than 7 pixels.

- Residual cut. The track residual in the local $\mathrm{x}$ direction has to be smaller than $1 \mathrm{~mm}$.

\subsubsection{Results}

Figure 6.25 shows two examples of the knee fit used to estimate the track position difference $y_{0}^{\prime}-y_{0}$. The beginning of the first pixel in the cluster is used for the charge measurement if the incidence angle is negative. The track position difference for tracks with negative angles is equal to the residual between the end of the last pixel and the knee position of the fit.

The approximated maximum and minimum track depth $t d^{\prime}$ is also estimated for each angle slice (see Figure 6.24). The outcome for the maximum track depth fit is shown as a function of the angle of incidence $\theta$ in Figure 6.26. The black points represent the results of the error function fit for the right side of the track depth distributions. The relation between the angle of incidence, the track depth, and cluster size is shown as a scatter plot. Only cluster sizes between 2 and 4 in the short pixel direction are included. The amount of statistics is not high enough for a cluster size larger than 4 . The approximated track depth $t d^{\prime}$ increases with the angle of incidence.

Equation 6.27 is used to calculate the depletion depth for each angle slice and the mean value is calculated for all slices. Two different sets of Monte Carlo simulations have been

\footnotetext{
${ }^{3}$ The transverse momentum of particle is defined as $p_{T}=|p| \sin (\Theta)$.
} 

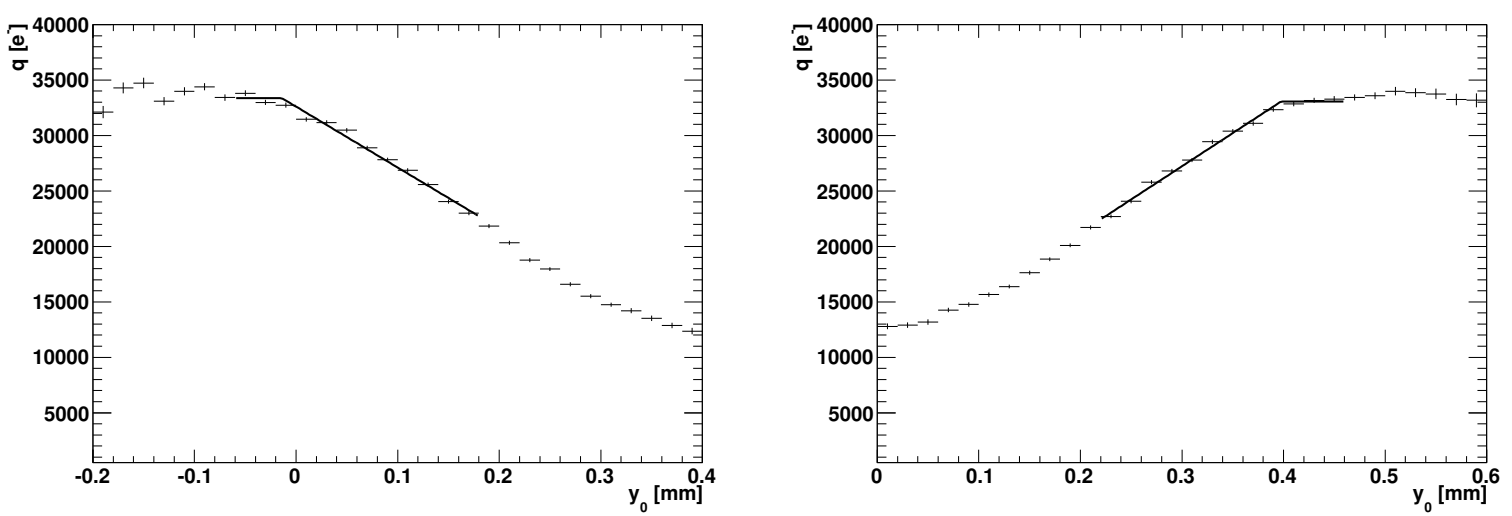

Figure 6.25.: Estimation of the difference between the real and approximated track position. The illustrated graphs include absolute track angles between 1.12 and 1.16 radians. Tracks with negative angles are shown in (a) and tracks with positive angles are shown in (b).

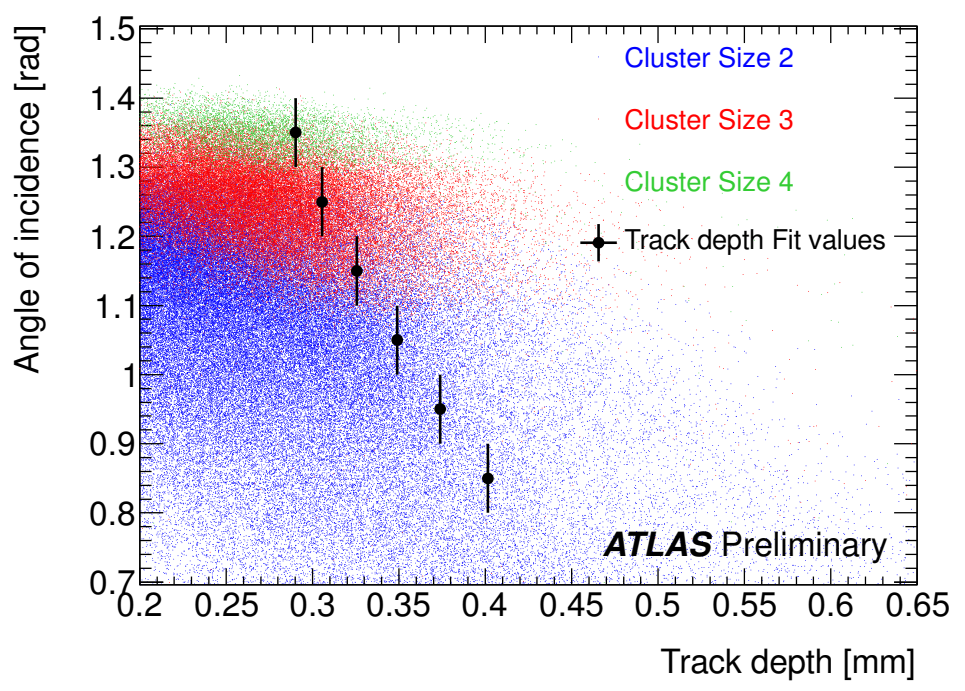

Figure 6.26.: Dependence between angle of incidence, track depth, cluster size and fit values 68 . 
produced in order to validate the measurement, one with a depletion depth of $D=250 \mu \mathrm{m}$ and a second one with $D=200 \mu \mathrm{m}$. The measured results are $D_{250}=(248 \pm 8$ (stat. $\left.)\right) \mu \mathrm{m}$ and $D_{200}=(201 \pm 6$ (stat. $\left.)\right) \mu \mathrm{m}$. The measurements are in agreement with the simulated depletion depths.

The measured depletion depths for runs 182516 and 187815 are:

$$
\begin{aligned}
& D_{182516}=(253 \pm 3(\text { stat. })) \mu \mathrm{m} \\
& \left.D_{187815}=(252 \pm 2 \text { (stat. })\right) \mu \mathrm{m} .
\end{aligned}
$$

Only the statistical uncertainty is quoted, while the systematic uncertainty is found to be $\sigma_{\text {sys }} \approx 10 \mu \mathrm{m}$. The expected sensor thickness is $S \approx 250 \mu \mathrm{m}$. Both results agree within the uncertainties and they are both consistent with the expected sensor thickness.

The measurement has been successfully adapted to estimate the depletion depth using collision data and it is fully data driven. Furthermore, with this method it is possible to compare test-beam and collision data measurements of the depletion depth. A second depletion depth measurement has been developed and is explained in the following section.

\subsection{Cluster Depth based Depletion Depth Measurement}

\subsubsection{Measurement Method}

The main idea of this method is to estimate the total travel length of the particle inside the pixel sensor in order to calculate the depletion depth. This method has been developed in addition to the track position measurement in order to decrease the minimum amount of statistics that are necessary for the depletion depth measurement.

As mentioned earlier, the fluence for inner modules is larger than for outer modules, thus, it is natural to illustrate the depletion depth as a function of the modules position. The variables in the track position measurement are functions of the track incidence angle (see Equation 6.27). Thus, it is necessary to split the available amount of statistics. This is not necessary for the new cluster depth based depletion depth measurement, which makes it better suited for monitoring purposes in the ATLAS pixel detector.

The total precision of both depletion depth measurements is similar, however, the track position measurement has the advantage that it can be used for a cluster size of two while the cluster depth method needs at least a cluster size of three pixels. Therefore, the cluster depth method has generally harsher geometric constraints and it is not necessarily suitable for measurements with small or fixed incidence angles, such as comparison measurements between test-beam and collision data.

A cluster with a size of three in the long pixel direction is used as an example to explain the measurement and the generalisation for larger clusters is made at the end. The total travel length $l=\sum_{i=1}^{3} l_{i}$ in the depleted region is a key value in this analysis, because it is directly related to the depletion depth of the sensor.

In this example a particle enters the sensor at the bottom, travels partially through the first pixel column, fully through the second pixel column in the center and again partially through the third pixel column before it exits the sensor at the readout side. A three dimensional example of the situation is shown in Figure 6.27. Only the depleted region is shown.

A projection in the $y-z$ plane of the active and inactive region is shown in 6.28(a), In the illustrated case, a larger depletion depth leads to a larger active area in the first pixel and thus 
increases $l_{1}$. The values needed in the calculation are the incidence angle $\varphi$ of the particle in the $R-\varphi$ plane, the incidence angle $\theta$ in the local y-z plane and the collected charge in each pixel of the sensor $Q_{i}(i=1,2,3)$.

It is possible that the particle travels through more than one pixel row, as is demonstrated in Figure 6.28(b). In this case the charge $Q_{3}$ is the sum of the two pixels that have been hit. The collected charge is separated for each pixel column and summed up for each pixel row in order to avoid Lorentz Angle effects.

Additional variables that are used in the derivation of the depletion depth formula are the track length $l_{y z}$ in the local y-z plane, the length of the track in each pixel column $l_{i}$, the total difference $\Delta x, \Delta y, \Delta z$ between the point where the track enters the depleted area $P_{1}$ of the sensor and the exit point on the pixel side of the sensor $P_{2}$ in local coordinates and the distance per pixel column $l_{y z i}, \Delta x_{i}, \Delta y_{i}, \Delta z_{i}$ where $i=1,2,3$.

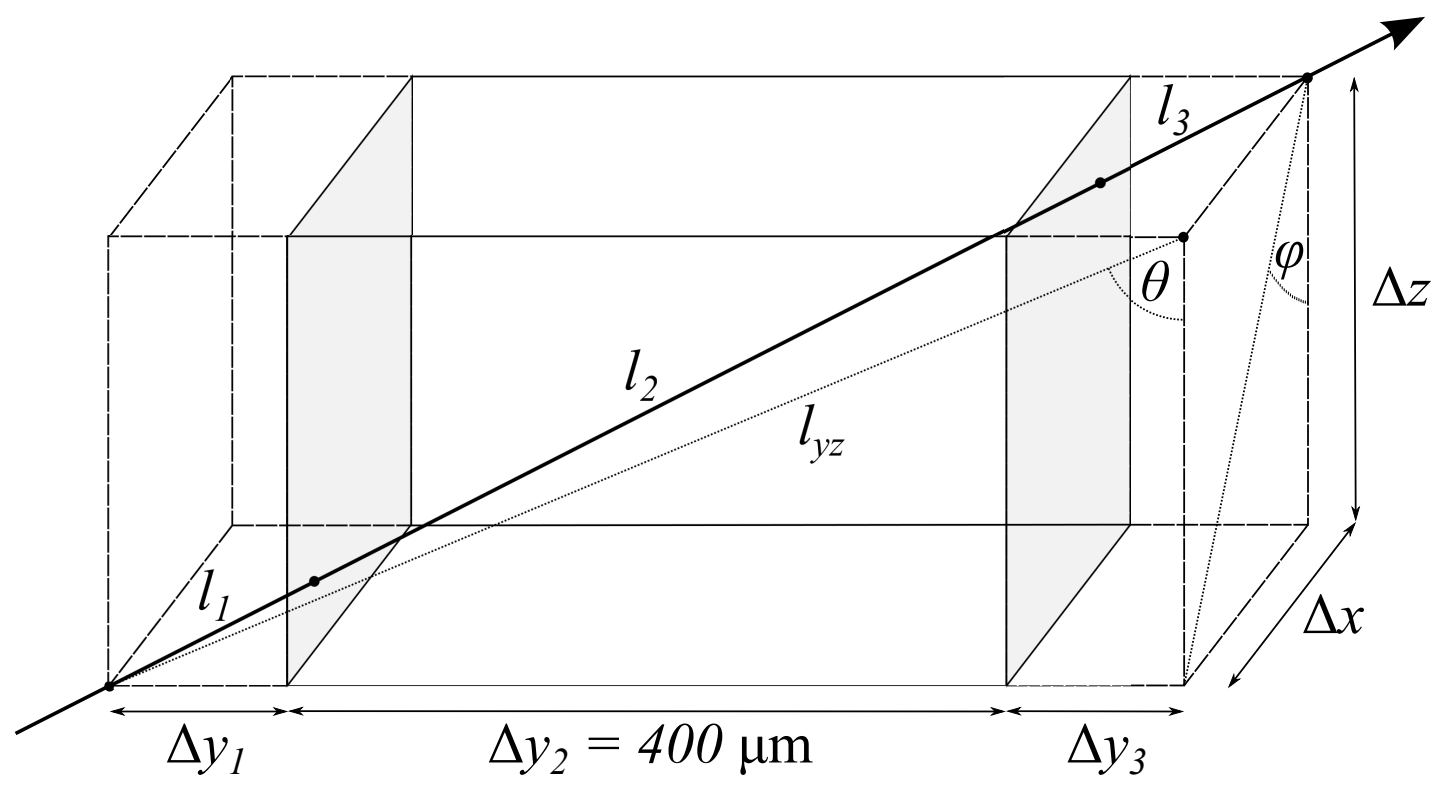

Figure 6.27.: Example of a particle passing through the pixel sensor. The depleted pixel region is shown in three dimensions. The track enters the depleted region at the left side travels through three pixel columns and leaves the sensor on the readout side.

A relationship between the depletion depth and the total track length $l$ and can be derived using the following trigonometric functions:

$$
\begin{array}{r}
\sin \theta=\frac{\Delta y}{l_{y z}}, \\
\tan \theta=\frac{\Delta y}{\Delta z}, \\
\tan \varphi=\frac{\Delta x}{\Delta z}, \\
l^{2}=l_{y z}^{2}+(\Delta x)^{2} .
\end{array}
$$




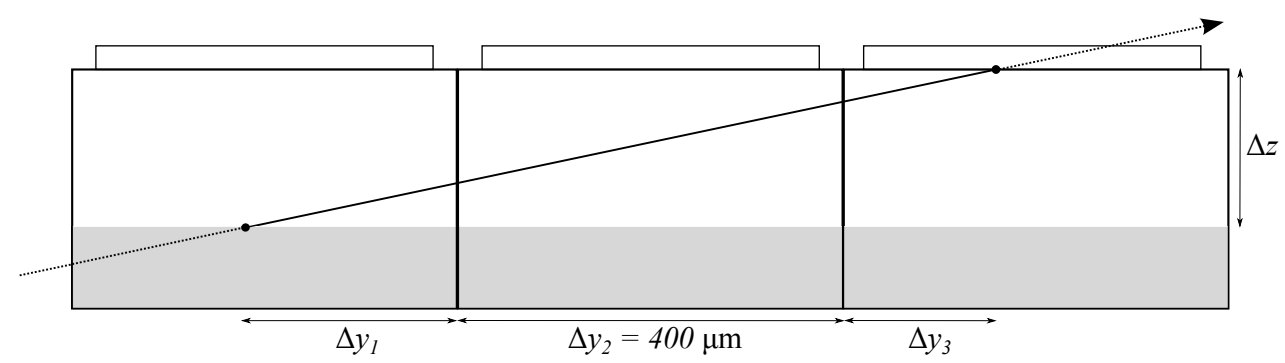

(a)

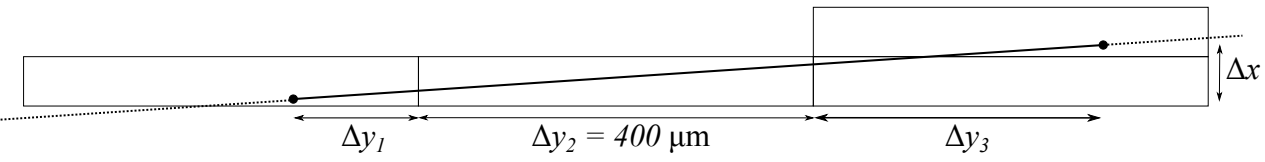

(b)

Figure 6.28.: The sensor is shown in the local $\mathrm{y}-\mathrm{z}$ plane (a) and from the top in the local $\mathrm{x}-\mathrm{y}$ plane (b). Tracks from collision data travel from the p-side of the sensor towards the readout-side. The solid line represents the track segment inside the depleted region of the sensor, while the dashed lines represent the track segments inside the undepleted region and outside the sensor.

Combining Equations 6.30-6.33 leads to:

$$
\begin{aligned}
& l^{2}=\Delta z^{2} \cdot \frac{\tan ^{2} \theta}{\sin ^{2} \theta}+\Delta z^{2} \tan ^{2} \varphi, \\
& l^{2}=\Delta z^{2} \cdot\left(1+\tan ^{2} \theta+\tan ^{2} \varphi\right) .
\end{aligned}
$$

The relationship can be written as a function of the track incidence angles and the total track length:

$$
\Delta z^{2}=l^{2} \cdot \frac{1}{1+\tan ^{2} \theta+\tan ^{2} \varphi} .
$$

The incidence angles are calculated in the reconstruction process and are known with very high precision. The only unknown value is the total track length $l$. It is calculated using the collected charge in each pixel. The track length $l_{2}$ in the center pixel can be calculated directly because the size of the pixel is known $\Delta y_{2}=P_{y}=400 \mu \mathrm{m}$.

Replacing $\Delta y$ with $\Delta y_{2}$ etc. in Equations 6.30-6.33 leads to:

$$
\begin{array}{r}
\sin \theta=\frac{P_{y}}{l_{y z 2}}, \\
\tan \theta=\frac{\Delta y_{2}}{\Delta z_{2}}, \\
\tan \varphi=\frac{\Delta x_{2}}{\Delta z_{2}}, \\
l_{2}^{2}=l_{y z 2}^{2}+z_{2}^{2} .
\end{array}
$$


With $\sin ^{2} \theta+\cos ^{2} \theta=1$ follows:

$$
l_{2}^{2}=P_{y}^{2} \cdot\left(1+\frac{1}{\tan ^{2} \theta}+\frac{\tan ^{2} \varphi}{\tan ^{2} \theta}\right) .
$$

For a Minimum Ionising Particle (MIP) the travelled distance in silicon is proportional to the average of the deposited charge. This information can be used together with collected charge in each pixel column to estimate the missing parts of the total track length:

$$
\begin{aligned}
& l_{1}=\frac{Q_{1}}{Q_{2}} \cdot l_{2}, \\
& l_{3}=\frac{Q_{3}}{Q_{2}} \cdot l_{2} .
\end{aligned}
$$

Combining the equations 6.36, 6.42, 6.43 and 6.44 one finds:

$$
\begin{aligned}
\Delta z^{2} & =\left(\frac{Q_{1}}{Q_{2}}+1+\frac{Q_{3}}{Q_{2}}\right)^{2} \cdot \frac{1+\frac{1}{\tan ^{2} \theta}+\frac{\tan ^{2} \varphi}{\tan ^{2} \theta}}{1+\tan ^{2} \theta+\tan ^{2} \varphi} \cdot P_{y}^{2}, \\
\Delta z & =\left(\frac{Q_{1}}{Q_{2}}+1+\frac{Q_{3}}{Q_{2}}\right) \cdot \frac{P_{y}}{|\tan \theta|} .
\end{aligned}
$$

The total height difference $\Delta z$ between the points $P_{1}$ and $P_{2}$ is called the cluster depth $c d$ and its most probable value is equal to the depletion depth $D$. The most probable value needs to be calculated, because the deposition of charge is a statistical process with a large variance. Thus, the calculated track length $l$ can vary a lot for a single track and it is not directly related to the depletion depth.

In order to derive the final formula it is necessary to take larger column sizes into account. Larger clusters only differ by having additional centre pixel and the track length for each of them is identical. This leads to:

$$
c d=\left(\frac{Q_{1}}{Q_{c}}+n+\frac{Q_{3}}{Q_{c}}\right) \cdot \frac{P_{y}}{|\tan \theta|},
$$

where $n=\Delta c o l-2$ is the number of centre columns in the pixel cluster for $\Delta$ col $>2 . Q_{c}$ is the mean charge of the center columns and it is equal to $Q_{2}$ for a column size of three.

\section{Most Probable Cluster Depth}

As mentioned, it is necessary to calculate the most probable value for the cluster depth. The calculated cluster depth is separated using the stave position of the hit pixel module (see Section 6.3). The separation is arbitrary and can be changed if needed. A histogram is created for each stave position and the calculated cluster depth is filled into the corresponding histogram. Thus, each histogram represents the cluster depth of a ring of modules with the same stave position in local z but different position in the $R-\varphi$ plane. An example of the cluster depth distribution at stave position -3 is given in Figure 6.29. Four different voltages have been applied in this example, during data taking.

The maximum value of the cluster depth is defined as the depletion depth. To calculate the depletion depth for each module ring a fit is used. The shape of the cluster depth distribution depends highly on the incidence angle and the bias voltage. In general, the cluster depth is 


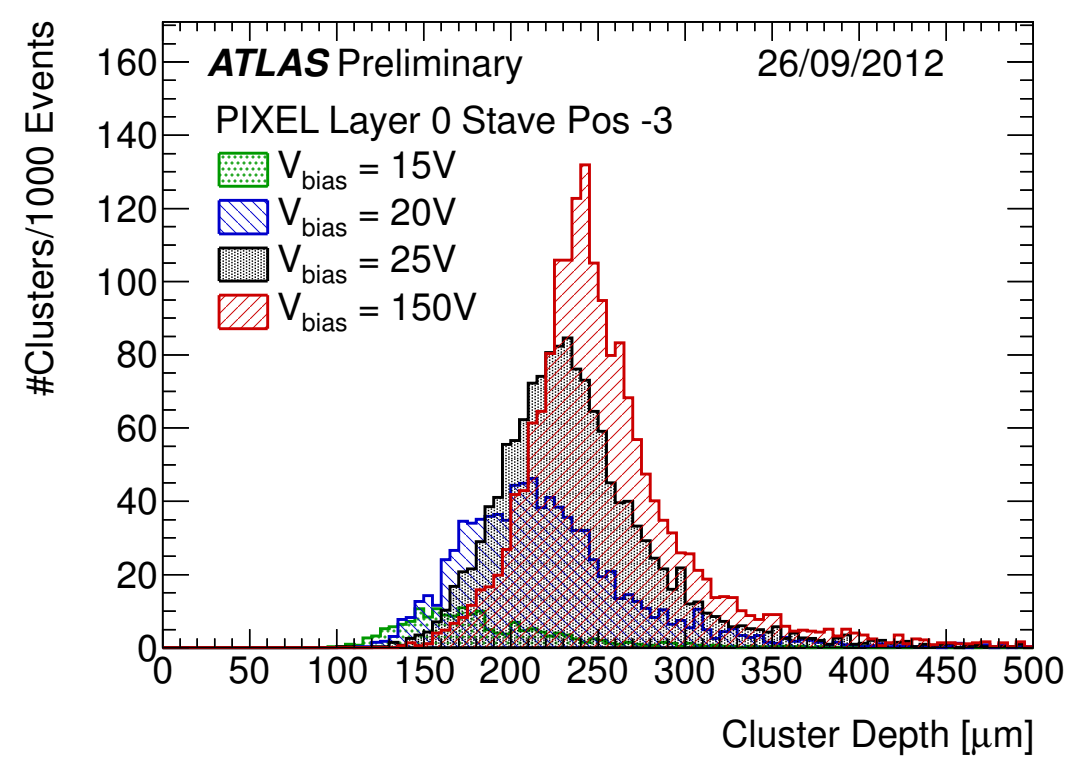

Figure 6.29.: Example of the cluster depth distribution shown for four different bias voltages. The distribution is a summation of all hit modules with module position -3 . The most probable value increases with increasing bias voltage and the shape of the distribution becomes more gaussian [68].

a convolution of a Landau and a normal function. However, only one of the two functions describes the shape very well, depending on the incidence angle. Therefore, it is possible to use two different fit functions to extract the most probable cluster depth instead of a convolution of both functions. The Landau distribution defines the shape of the cluster depth, if the reverse bias voltage is smaller than $15 \mathrm{~V}$ while the shape is described by the normal distribution at larger bias voltages.

Therefore, the following fit function is used in the first case to calculate the depletion depth:

$$
p(x)=a \cdot \phi\left(\frac{x-m p v}{\sigma}\right)=a \cdot \phi(\lambda),
$$

where $a, m p v, \sigma$ are parameters determined by the fit. The first scale factor is $a, m p v$ approximates the most probable value, and $\sigma$ is the second scale factor, since the mean and the variance of the Landau distribution are undefined. $\phi(\lambda)$ is defined by the following complex integral:

$$
\phi(\lambda)=\frac{1}{2 \pi i} \int_{c-i \infty}^{c+i \infty} \exp (\lambda s+s \ln s) d s,
$$

where $c$ does not change the result and it can be any positive real number. As mentioned previously a normal distribution is used as the fit function, if the bias voltage is set to values larger than $15 \mathrm{~V}$ :

$$
f(x)=a \cdot \exp \left(-\frac{(x-\mu)^{2}}{2 \sigma^{2}}\right),
$$

where $a, \mu, \sigma^{2}$ are fit parameters. The scale factor is $a, \mu$ is the mean value, and $\sigma^{2}$ is the variance. Non Gaussian tails are present in each cluster depth histogram. Therefore, the fit is 
restricted to the area around the maximum of the distribution. The estimation of the depletion depth is influenced by systematic uncertainties, which are described in Subsection 6.6.3.

\subsubsection{Validation with Monte Carlo Simulations}

The cluster depth method has been validated on two Monte Carlo samples. One with a full depletion of $S=250 \mu \mathrm{m}$ and a second one partially depleted with a depth of $S=200 \mu \mathrm{m}$. Results for layer 0 are presented in Figure 6.30. Both results are consistent with the simulated depletion depth within the uncertainties. The true depletion depth is underestimated by less than $5 \mu \mathrm{m}$.

The depletion depth measurements show a slight dependence of the module position on a stave. The track incidence angle $\theta$ is overall smaller for modules closer to the interaction point leading to shorter track lengths in the pixel detector. This leads to a slightly larger impact of the systematic uncertainties.

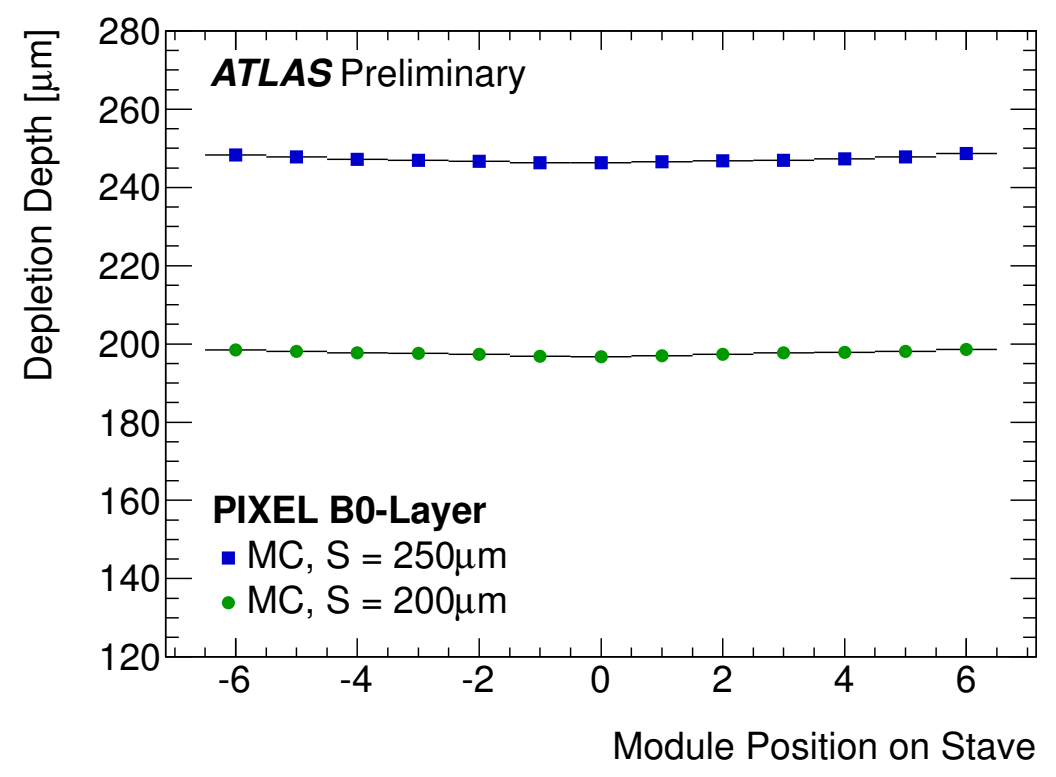

Figure 6.30.: Depletion depth as a function of the module position on stave. Monte Carlo data with two different depletion depths have been produced in order to validate the method. Only the statistical uncertainty of the fit is shown [68].

\subsubsection{Sources of Systematic Uncertainties}

The cluster depth based depletion depth measurement is mainly affected by two systematic uncertainties, the charge collection uncertainty and the underestimation of the track length due to threshold effects. As mentioned earlier, the charge collection residual is overall equal to $\sim 400$ electrons, while the standard deviation can be as large as 1000 electrons. The exact deviation depends on the total amount of the collected charge, however the exact values are not known. All charges $Q_{i}$ in Equation 6.47 are affected by this uncertainty. Since the exact dependency is unknown, a total correlation of the systematic uncertainties is assumed and the mean of the residual is used as the value for all uncertainties $\sigma_{Q_{i}}$. 
The impact of the uncertainty is then approximated by:

$$
\sigma_{\mathrm{sys}}=\sum_{i=1}^{3} \frac{\partial c d}{\partial Q_{i}} \sigma_{\mathrm{sys}}\left(Q_{i}\right)=\left(\frac{\sigma_{\mathrm{sys}}\left(Q_{1}\right)+\sigma_{\mathrm{sys}}\left(Q_{3}\right)}{Q_{2}}-\frac{Q_{1}+Q_{3}}{Q_{2}^{2}} \cdot \sigma_{\mathrm{sys}}\left(Q_{2}\right)\right) \cdot \frac{400 \mu \mathrm{m}}{|\tan \theta|},
$$

With an average charge of about 30000 electrons deposited in one pixel column a systematic uncertainty of $\sigma_{\text {sys }} \approx 10-20 \mu \mathrm{m}$ is found depending on the track incidence angle $\theta$. The impact of the charge collection residual is expected to be the dominating effect and thus limiting the precision of the measurements as long as the sensors are not heavily irradiated. However, two additional and smaller uncertainties have been identified.

The second uncertainty is the underestimation of the track length due to threshold effects. If the entry or exit point of the track is very close to a pixel edge, then it is possible, that the track segment in the pixel is very short. Thus, the pixel might not collect enough charge to reach its discriminator threshold. In these cases the total track length is underestimated. The threshold has been tuned to a mean value of $\sim 4000$ electrons [77]. This roughly corresponds to a minimum track length in the sensor of $40 \mu \mathrm{m}$ to $50 \mu \mathrm{m}$. With Equation 6.36 follows an overall underestimation of the cluster depth between $\Delta c d \approx 6-30 \mu \mathrm{m}$. However, only a small subset of tracks are affected by this uncertainty.

The mean value of this underestimation is a function of the track incidence angle and thus leads to the creation of a second peak in the cluster depth distribution. The cluster depth fit might be influenced, depending on the position of the peak, the standard deviation, and the amount of tracks that are affected. Only a small subset of all tracks enter the sensor in such a way that the threshold is not reached in a certain pixel and no clear second peak is visible in the cluster depth distributions (see Figure 6.33). Thus, the impact of the threshold uncertainty is significantly smaller than the impact of the charge collection uncertainty.

A third systematic uncertainty is introduced by the fit of the cluster depth distribution. As mentioned earlier the shape of the distribution is a combination of a Landau distribution and a normal distribution. The contributions of both distribution depend highly on the depletion depth of the sensor, however a small asymmetric Landau contribution is always present. The depletion depth is extracted by a fit of the most probable cluster depth. However, the most probable value of the collected charge changes if the sensor thickness changes (see Chapter 2), which also has an influence on the maximum of the cluster depth. As a result the relationship between the track segments $l_{1}, l_{2}$ and $l_{3}$ is not entirely linear as assumed. The additional change in the depletion depth measurement is expected to be of the order of $\sigma_{\mathrm{sys}} \approx 5 \mu \mathrm{m}$.

In addition, charge trapping and the distribution of the electric field needs to be regarded in the future as soon as the sensors are heavily irradiated. The impact of the change in the electric field is similar for the track position and the cluster depth measurement. Trapping has an impact on the depletion depth measurement, because charge carriers coming from track segments close to the backside are lost which leads to a change in the charge weighted cluster depth distribution. However, these influences lead to a measurement of the effective charge collection depth which is also an important factor, as discussed previously. The possible impact of trapping centres has been discussed in detail in Subsection 6.5.2.

\subsubsection{Geometric Constraints}

As mentioned earlier, measurements are projected in the long pixel direction in order to avoid the impact of the Lorentz angle. However, a consequence of the projection in the long pixel direction and the minimum cluster size of three directions are additional geometric constraints. 
The exact values depend on the depletion depth of the pixel sensors and the position of the collision point. The constraints for tracks originating from the collision point are illustrated in Figure 6.31. The sensitive region of the sensor decreases with time and so does the cluster size. A minimum column size of three can, therefore, only be achieved for tracks with higher incidence angles and modules located in the centre are not covered any longer.

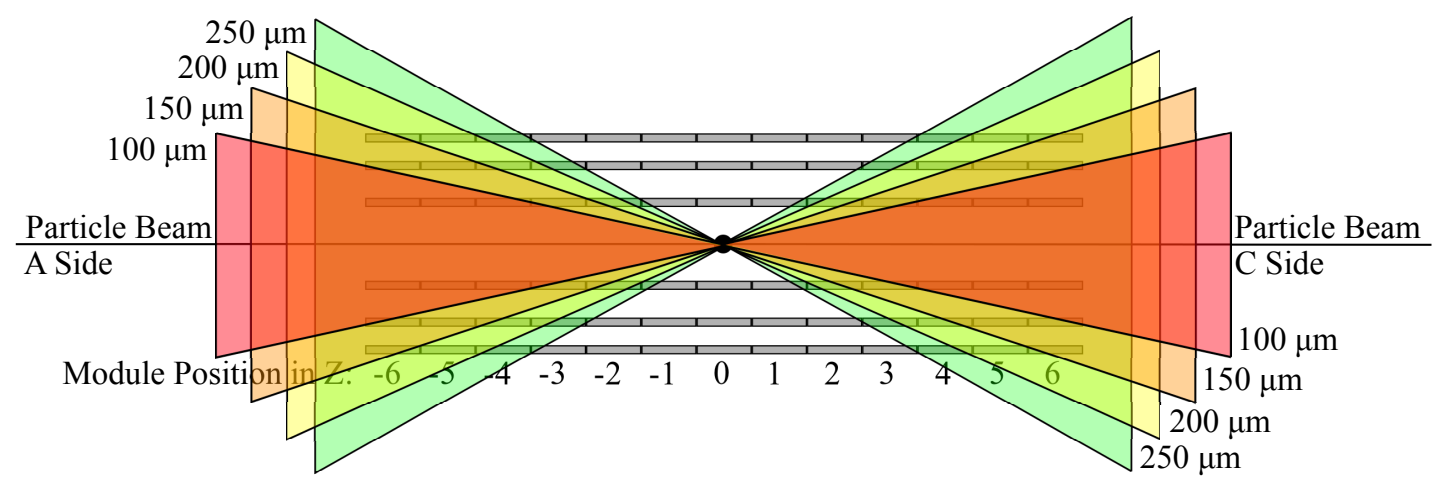

Figure 6.31.: Analysis area depending on the depletion depth of the pixel sensor. The corresponding value of the depletion depth is indicated in $\mu \mathrm{m}$ at the edge of each analysis area. A smaller depletion depth causes smaller column size of the cluster. Less modules are covered for smaller depletion depths.

The nominal interaction is at the centre of the pixel detector as illustrated in Figure 6.31 and hard scattering events originate from a single proton-proton collision in an event. In addition to that, several secondary low $p_{T}$ interactions also occur independently while the two bunches collide (see also Section 7.4). These so called pile-up events are in general not of interest for physics analysis, however, this is not the case for the depletion depth measurement and tracks from pile-up events are thus used in the analysis.

$Z_{0}$ is defined as the point of closest approach for tracks coming from the interaction point. It is the coordinate measured parallel to the beam and its centre is in the middle of the pixel detector. An example of the distribution is shown in Figure 6.32 and a normal distribution is fitted to the data. The estimated mean value is $\mu=(-0.933 \pm 0.003) \mathrm{mm}$ and the standard deviation is $\sigma=(54.529 \pm 0.003) \mathrm{mm}$. The origins of the tracks are distributed around the nominal collision point. Therefore, about one to one and a half additional modules are covered in both directions by the cluster depth measurement compared to tracks only coming from the nominal collision point. This means that the geometric constraints in reality are not quite as harsh as they are indicated in Figure 6.31. However, the cluster position based depletion depth measurement is still only applicable for large values of the depletion depth in the outer layers of the detector. The coverage of Layer 0 is significantly larger and measurements can still be carried out at a depletion depth of $100 \mu \mathrm{m}$.

An approximation of the covered detector area is calculated. The impact of pile-up events is included by assuming that 2 more modules are covered in addition to the area that is covered in Figure 6.31. Table 6.4 shows the coverage of the detector layers for four different depletion depths. 


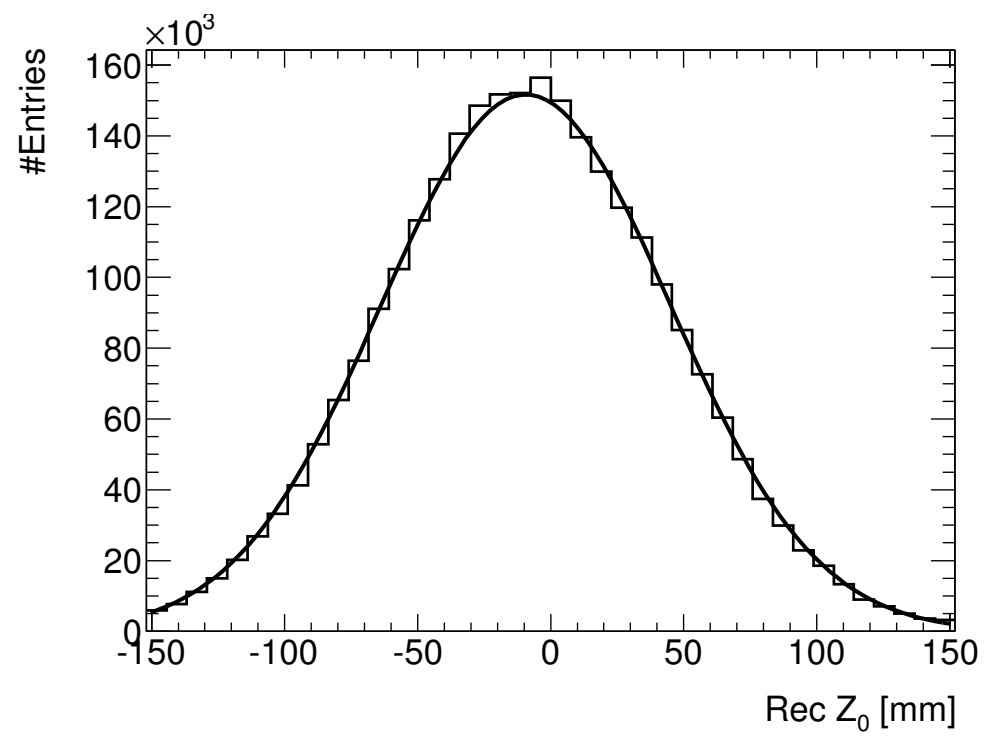

Figure 6.32.: Reconstructed $Z_{0}$ distribution and Gaussian fit. The fit results are $\mu=(-0.933 \pm$ $0.003) \mathrm{mm}$ and $\sigma=(54.529 \pm 0.003) \mathrm{mm}$.

\begin{tabular}{|l|c|c|c|c|}
\hline Depletion Depth: & $250 \mu \mathrm{m}$ & $200 \mu \mathrm{m}$ & $150 \mu \mathrm{m}$ & $100 \mu \mathrm{m}$ \\
\hline Layer 0: & $95 \%$ & $89 \%$ & $80 \%$ & $62 \%$ \\
Layer 1: & $78 \%$ & $68 \%$ & $52 \%$ & $20 \%$ \\
Layer 2: & $63 \%$ & $50 \%$ & $27 \%$ & $0 \%$ \\
\hline
\end{tabular}

Table 6.4.: Approximated acceptance of the cluster depth measurement. Pile-up events have been included by assuming that two more modules are covered. The covered area of each barrel layer is shown for four different depletion depth values.

\subsubsection{Data Samples and Quality Cuts}

In order to be able to measure the relation between the applied reverse bias voltage and the depletion depth, four different depletion depth scans have been taken in Layer 0 during data taking, while two scans have been taken in Layer 1 . The dates and runs of the high voltage scans are summarised in Table 6.5. The bias voltage is only changed in one layer at a time and the applied voltage of the other layers is $150 \mathrm{~V}$. The last scan had to be taken during the course of several days, because various unexpected beam dumps prevented its completion.

\begin{tabular}{|c|c|c|}
\hline Run & Date & LumiBlocks \\
\hline 206253 & 02.07 .2012 & $52-156$ \\
211522 & 26.09 .2012 & $62-171$ \\
213754 & 01.11 .2012 & $1260-1350$ \\
218036 & 22.01 .2013 & $45-70$ \\
218168 & 24.01 .2013 & $392-422$ \\
218179 & 25.01 .2013 & $600-652$ \\
218436 & 29.01 .2013 & $264-377$ \\
\hline
\end{tabular}

Table 6.5.: Summary of runs during which the high voltage scans have been performed. 
Quality cuts are applied to improve the precision of the measurement. First a track selection is done in order to reject tracks with high uncertainties on their track parameters. In addition, cuts are performed on the cluster level. All applied cuts are summarised in the following:

- Momentum cut. Tracks need a minimum transverse momentum of $p_{T}>1 \mathrm{GeV}$.

- Sufficient number of hits. Tracks need more than one Pixel hit and at least 7 SCT hits.

- Isolation cut. There is no other cluster on the same module within $1.5 \mathrm{~mm}$.

- No ganged pixels and no long pixels. Ganged pixels and long pixels between the FE chips are excluded.

- First rows and columns. In order to avoid edge effects the cluster should not contain any cells belonging to the first or the last two rows and columns of pixels.

- Residual cut. The track residual has to be smaller than $0.5 \mathrm{~mm}$.

- Cluster size. The cluster size in the local x direction has to be smaller than 5 pixels and the cluster size in local y direction has to be larger than 2 pixels.

\subsubsection{Results}

\section{Cluster Depth}

Examples of the cluster depth distributions for all four bias voltage scans are shown in Figure 6.33. The module position on stave is -3 in all cases. Different values of the bias voltage were used during the different scans, because the depletion depth is changing constantly and due to time constraints it is only possible to use a very limited set of voltages. Each depletion depth scan has a different amount of statistics, thus, the cluster depth distributions are normalised in order to be able to compare different scans.

An evolution of the depletion voltage can already be seen in the graphs. For $150 \mathrm{~V}$ the maximum of the cluster depth distribution is at the same place for all four cases, because the detector is always fully depleted at this voltage. However, in the first case the detector is still nearly fully depleted at $20 \mathrm{~V}$, while it is clearly not fully depleted at this voltage later on. In order to measure a distribution that is similar to the first one at $20 \mathrm{~V}$ the voltage needs to be increased over time. This is why a shift in the cluster depth over time is a direct indication of the change in depletion voltage. Measurements of this value are discussed in more detail in the next paragraphs.

The normal operation voltage of the detector is $150 \mathrm{~V}$. It is clearly visible that not only the maximum value, but also the shape of the cluster depth distribution is identical for the maximum bias voltage, while the shape changes for smaller bias voltages.

\section{Collision Data Results}

Figure 6.34 shows the depletion depth as a function of the stave position for all four bias voltage scans. The first scan was taken right after the last cross-talk scan started to indicate typeinversion in the first detector layer. A close-up of the region with high bias voltages for the same bias scans are shown in Figure 6.35. The last three scans have been taken at regular intervals after type-inversion in order to monitor the development of the depletion depth and to extract the depletion voltage after type-inversion (see Section 6.7). 


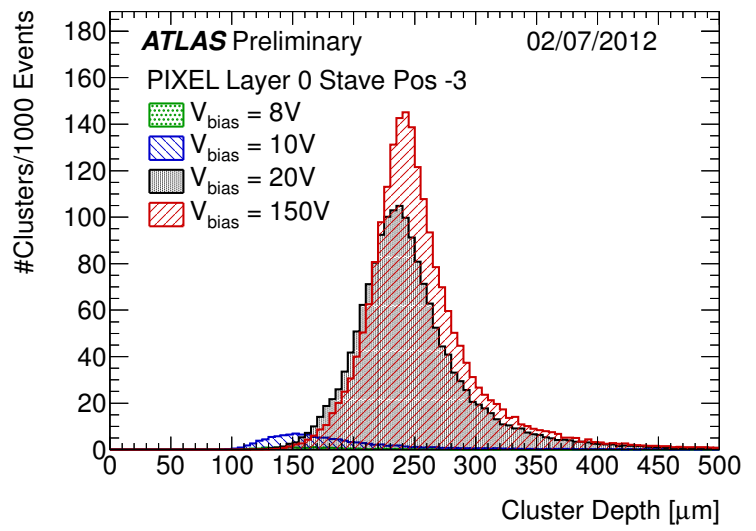

(a)

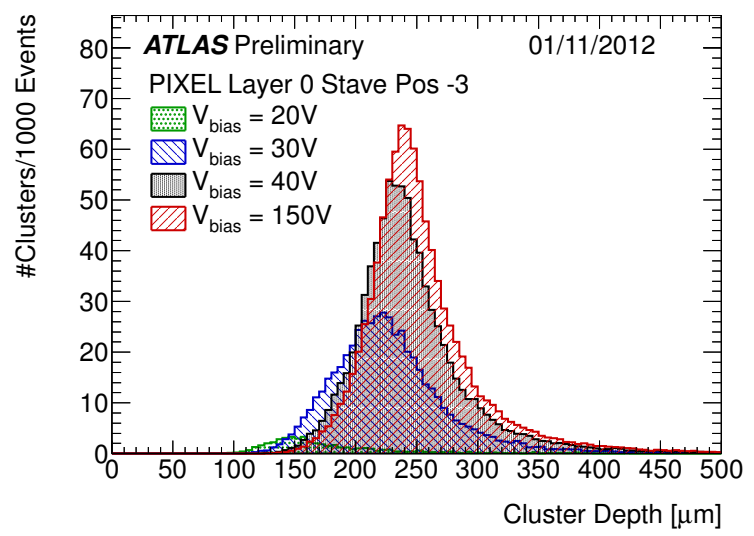

(c)

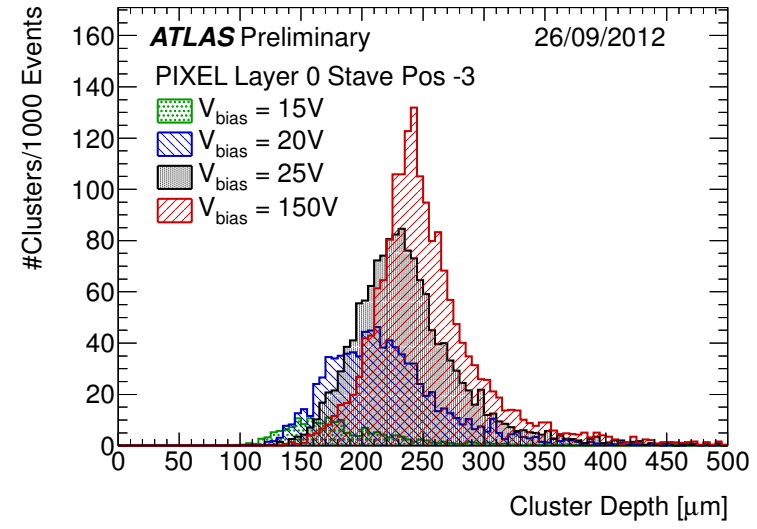

(b)

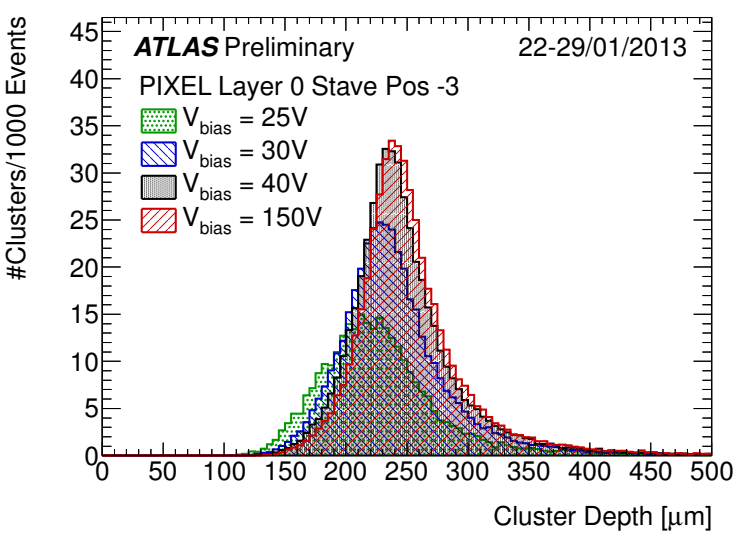

(d)

Figure 6.33.: Cluster depth distribution shown for all four bias voltage scans. The most probable value of the cluster depth increases with increasing bias voltage and it decreases with time for a given voltage. Only the module ring at the stave position of -3 in layer 0 is shown [68].

The first layer of the pixel detector started to undergo type-inversion at the beginning of July 2012 and it was still in the process of type-inversion when the first scan was taken. The cluster depth based depletion depth measurement always works for fully depleted sensors and only for partially depleted modules after type-inversion. Partially depleted modules that are not-type inverted always appear to be fully undepleted. Thus, only the subset of pixel modules that already underwent type-inversion can be taken into account as long as sensors are not fully depleted. Figure 6.34(a) shows the depletion depth for the first scan on the 02.07.2012. A more detailed zoom for large bias voltage is also shown in Figure 6.35(a),

The depletion voltage is at its minimum during type-inversion and the depleted region grows very fast with increasing bias voltage. The depletion depth grows rapidly up to a voltage of $20 \mathrm{~V}$ and the increase slows down considerably above a bias voltage of $30 \mathrm{~V}$. Thus, it can be concluded that the effective depletion voltage lies between 20 and $30 \mathrm{~V}$. 


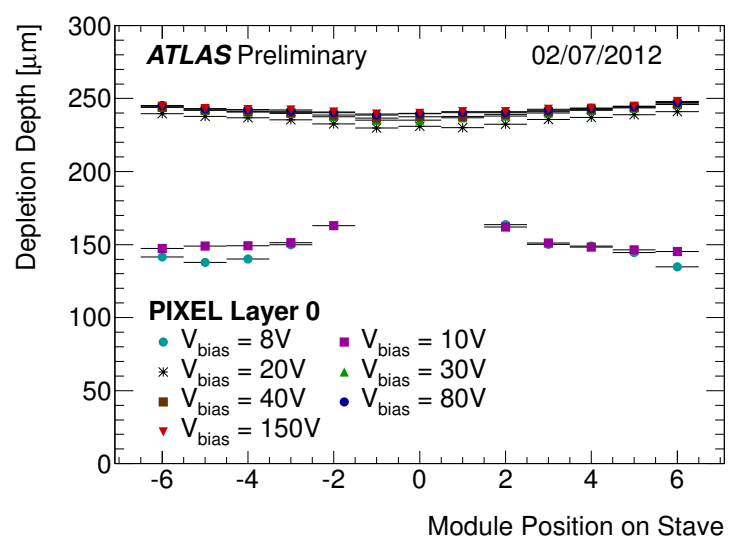

(a)

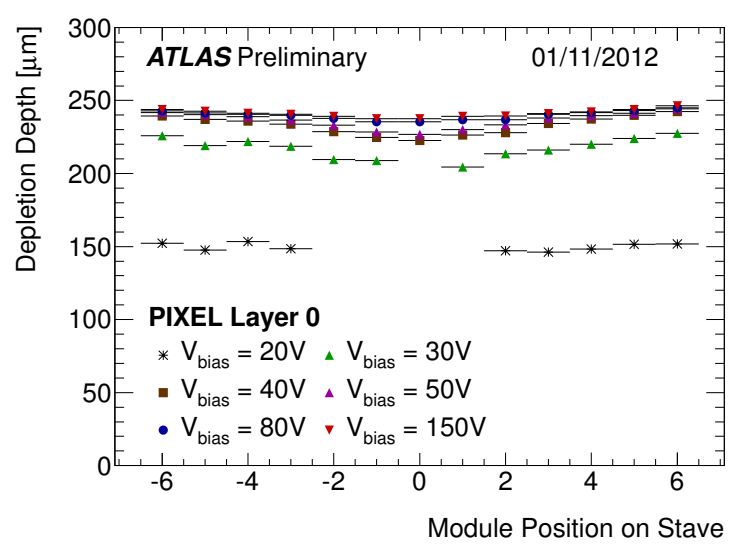

(c)

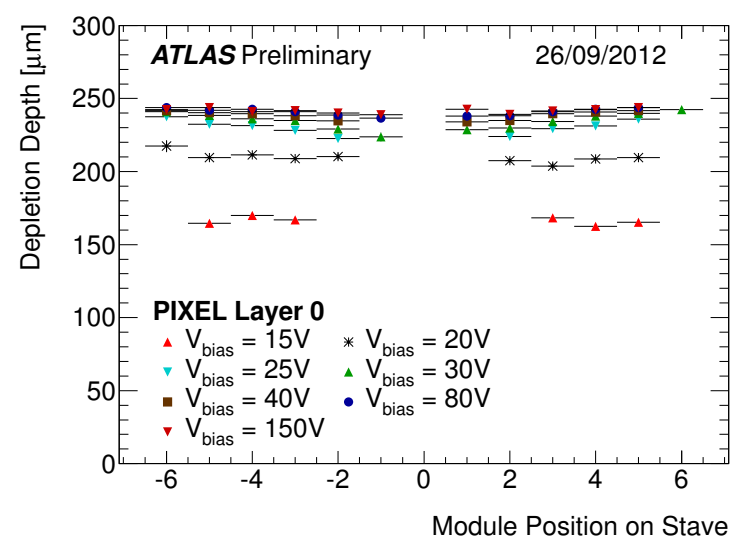

(b)

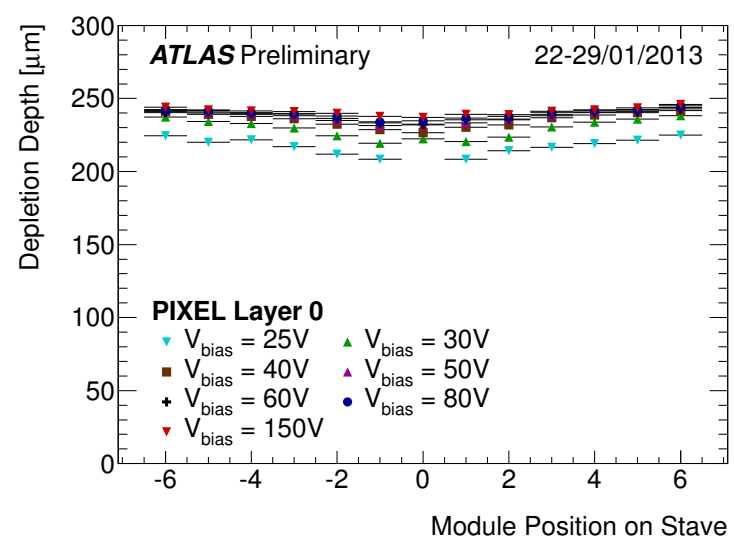

(d)

Figure 6.34.: Depletion depth shown as a function of the module position on stave. All four bias voltage scans are shown. The first scan was taken right after type-inversion of layer 0 . The amount of statistics is too low for measurements in the central region of the detector for small values of the bias voltage 68 .

It is expected that the depletion voltage increases after type-inversion and thus it is expected that the depletion depth decreases with time for a fixed bias voltage. This general trend can be observed in the depletion depth scans. While the depletion depth for a voltage of $20 \mathrm{~V}$ is between 240 and $230 \mu \mathrm{m}$ it has already decreased to $\approx 210 \mu \mathrm{m}$ in the second and to $\approx 150 \mu \mathrm{m}$ in the third scan. The last scan has been taken after the Christmas break where beneficial annealing has occurred.

The largest systematic uncertainty in the depletion depth measurement is caused by the charge collection uncertainty of the pixel detector as is explained in Subsection 6.6.3. The impact of the systematic uncertainty depends on the incidence angle of the track and is thus in general larger for pixel modules close to the interaction point. The impact of the systematic uncertainties can be evaluated by looking at fully depleted sensors. The measurement should be constant and identical for every module position on stave if the bias voltage is larger than the depletion voltage. A bias voltage of $150 \mathrm{~V}$ is in all four cases larger than the depletion voltage. 


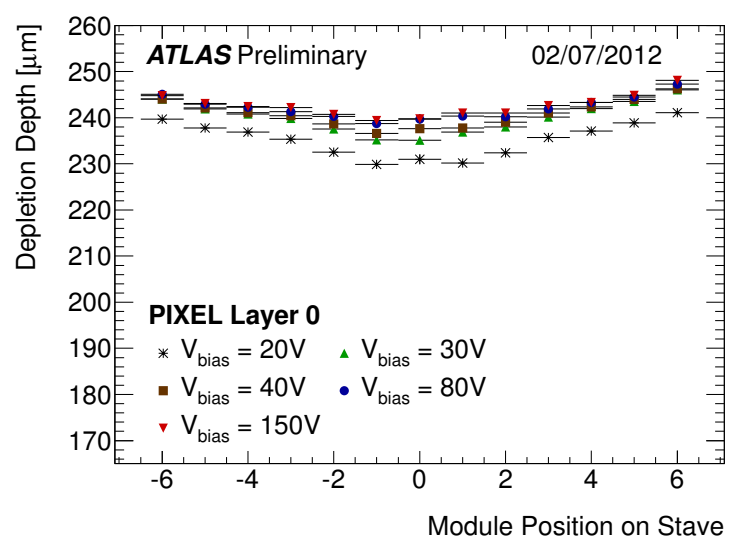

(a)

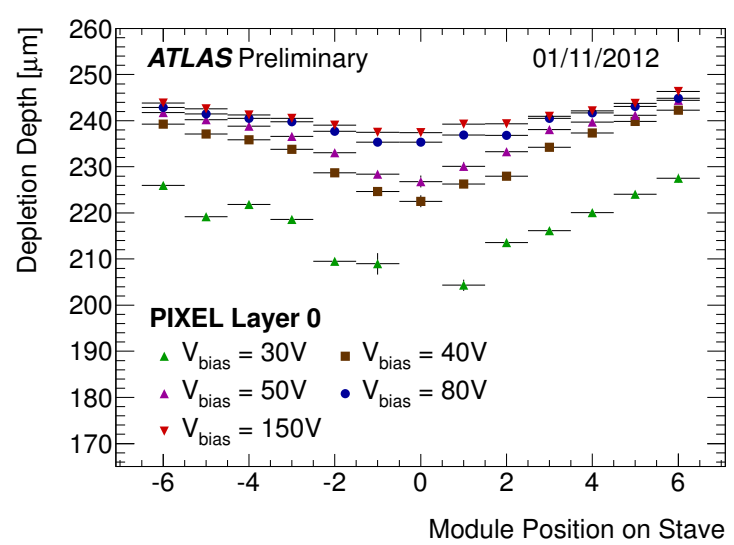

(c)

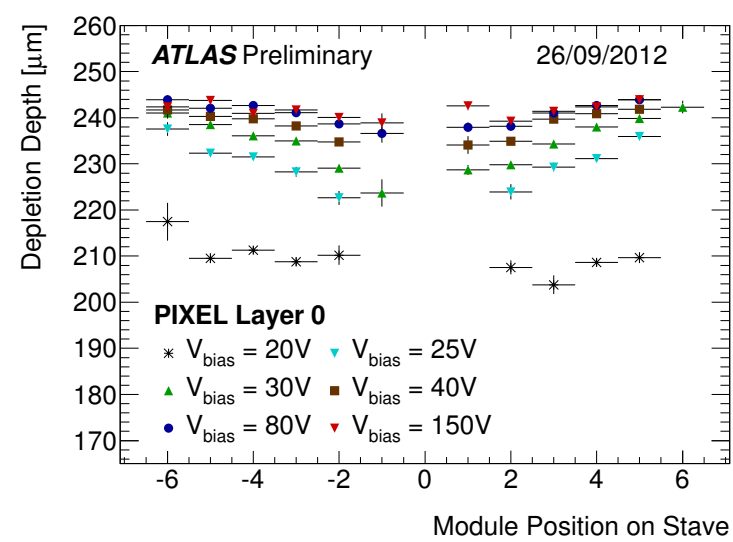

(b)

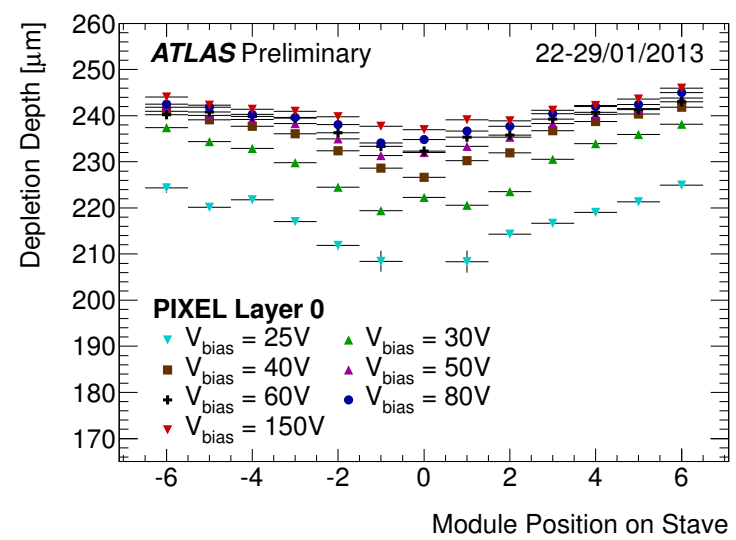

(d)

Figure 6.35.: Depletion depth as a function of the module position on stave. A close-up of the region with larger bias voltages is shown [68].

The measured depletion depth is a function of the module position even if it is fully depleted, as can be seen in Figure 6.35(a).

The discrepancy between the real sensor thickness of $\approx 250 \mu \mathrm{m}$ and the measured sensor thickness in collision data is in general slightly larger than the measured difference in Monte Carlo data. However, all measurements are within the estimated systematic uncertainties of 10 to $20 \mu \mathrm{m}$.

When the depletion depth starts to decrease additional effects need to be taken into account. Modules close to the interaction point are exposed to a higher particle flux and thus show in general more radiation damage effects. This effect could already be observed before typeinversion (see Figure 6.16). The change in the depletion voltage is $\approx 3 \mathrm{~V}$ larger for the inner modules than for the outer modules. After type-inversion inner modules will thus have a larger depletion voltage than outer modules and therefore, the depletion depth is smaller for a fixed bias voltage. However, this effect is overlapped by the systematic uncertainty which is also larger for inner modules and thus it is impossible to quantify the difference in depletion voltage after type-inversion. 
An interesting result is found for very low bias voltages during type-inversion. The measured depletion depth is larger for inner modules than for outer modules. In general it is expected that the depletion depth is smaller for inner modules at a given bias voltage due to the above described effects. However, as mentioned, only a part of the detector is already type-inverted and only this subset of modules can be measured in this case.

In addition, during type-inversion it is possible that the effective doping concentration in the sensor bulk is right at the edge of changing the sensor from n-type to p-type silicon. During this process part of the sensor can already be type-inverted while some part is not. This should have no effect on the detector performance, as long as the sensor is fully depleted. However, the operation of a silicon detector without a strict border between the n- and p-type silicon at very low bias voltages can create unexpected behaviour.

Depletion voltage measurements before type-inversion reveal a large influence of beneficial annealing on the sensor properties after each cooling stop. A similar behaviour is observed after type-inversion. Beneficial annealing leads to an increase of the depletion depth at a fixed bias voltage. The detector was shut-down during the Christmas break at the end of 2012. The difference between the depletion depth at a bias voltage of $30 \mathrm{~V}$ is shown in Figure 6.35(c) and Figure 6.35(d), The depletion depth values lie between $D \approx 230 \mu \mathrm{m}$ and $D \approx 210 \mu \mathrm{m}$ at the beginning of November. While they have increased to values between $D \approx 240 \mu \mathrm{m}$ and $D \approx 220 \mu \mathrm{m}$ at the end of January.

Layer 1 was expected to undergo type-inversion at the beginning of November 2012. Thus, depletion depth scans have been performed for Layer 1 from the beginning of November. The results are shown in Figure 6.36. The first scan is taken during or right after type-inversion. The depletion depth distribution for Layer 1 shows the same behaviour as the distribution for Layer 0 during type-inversion. The depletion depth values are larger for inner modules and a low bias voltage. The second scan after type-inversion shows the expected behaviour. The innermost modules are not included in this measurement due to the strict geometric constraints of the measurement (see Subsection 6.6.4).

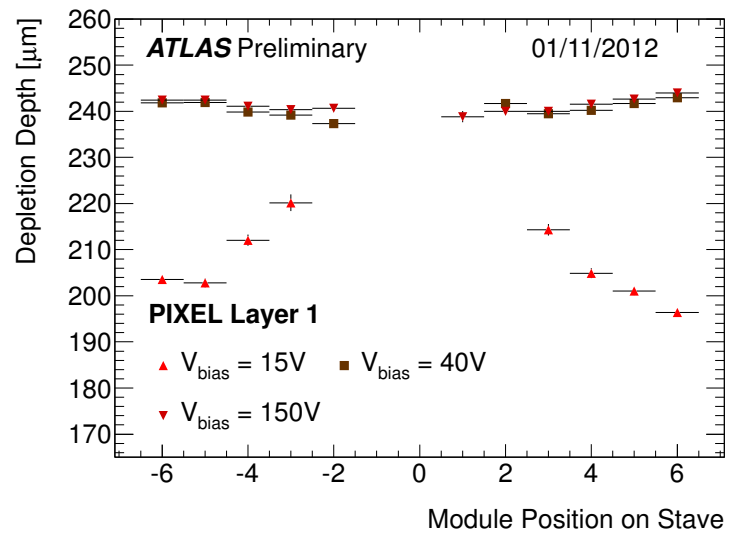

(a)

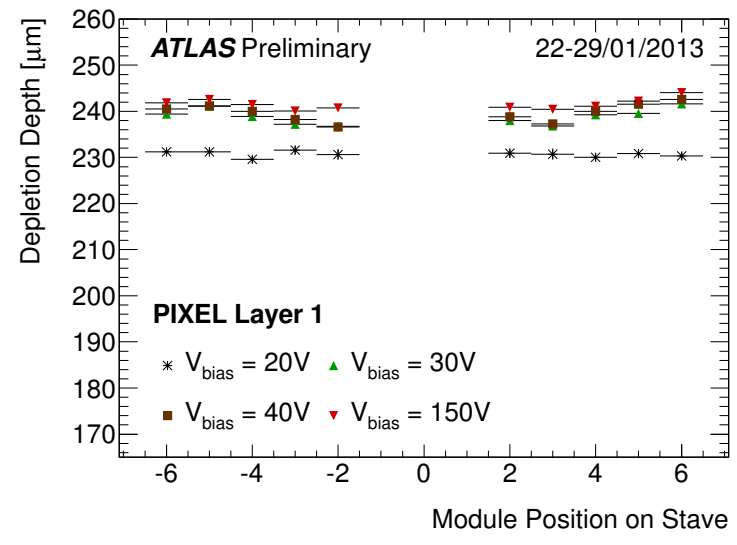

(b)

Figure 6.36.: Depletion depth shown as a function of the module position on stave. Layer 1 is shown. Layer 1 underwent type-inversion in November 2012. The first scan was taken right after type-inversion 68 . 


\subsection{Measurement of the Effective Depletion Voltage after Type-Inversion}

\subsubsection{Measurement Method}

The effective depletion voltage after type-inversion is extracted from the depletion depth scans. The cluster based depletion depth measurement has been used for the estimation of the effective depletion voltage and results are shown for layer 0 only. In order to be able to compare the depletion voltage measurements to the module prediction it is necessary to calculate an average for the whole detector layer. Thus, a mean value for the depletion depth is calculated for the entire detector layer at each bias voltage. Figure 6.37 shows the relation for the scan taken at the 26.09.2012. All scans are shown in Figure A.6.

The measurement of the depletion depth depends on the stave position of the corresponding modules. As discussed in Subsection 6.6.3 the systematic uncertainty of the cluster based depletion depth measurement has been estimated to be approximately $10-20 \mu \mathrm{m}$. Thus a dependence of the depletion depth on the module position can only be seen if the depletion depth difference between inner and outer modules is larger than $10 \mu \mathrm{m}$. As it can be seen in Figure 6.35 the difference between in inner and outer modules is roughly $10-15 \mu \mathrm{m}$ for most bias voltages and is thus in the same range. Therefore, the calculation of a mean value for the whole detector layer is still feasible after type-inversion. This relationship will change in the future with increasing radiation damage and it might become necessary to measure the effective depletion voltage as a function of the module position.

The depletion depth grows very fast with increasing bias voltage until a certain point, after which the depletion depth increase is very small (see Figure 6.37). The change in the slope occurs at $\sim 98 \%$ of the sensor thickness. Thus, the effective depletion voltage after type-inversion is defined at the point $D$ where the depletion depth function changes its slope drastically:

$$
D_{v}=D_{\max }-5 \mu \mathrm{m} .
$$

$D_{\max }$ is the maximum depletion depth, it is consistent with the sensor thickness within the estimated uncertainties. A linear fit is used to find the exact position of the effective depletion voltage:

$$
f(x)=a \cdot x+b,
$$

where $\mathrm{a}$ and $\mathrm{b}$ are fit parameters. The closest points below and above the approximated position of the depletion voltage are taken for the measurement. They are chosen by measuring the distance between $D_{\max }-5 \mu \mathrm{m}$ and the data point. The boundaries of the fit are $-2.5 \mathrm{~V}$ below and $+2.5 \mathrm{~V}$ above the two chosen data points.

The effective depletion voltage $x=V_{\text {depl }}$ is found at the point where $f(x)=D_{v}$, as mentioned earlier. Thus, the effective depletion voltage is calculated to be:

$$
V_{\mathrm{depl}}=\frac{D_{\max }-5 \mu \mathrm{m}-b}{a},
$$

and the error is calculated by:

$$
\sigma=\sqrt{\left(\frac{D_{\max }-b}{a^{2}} \cdot \sigma_{a}\right)^{2}+\left(\frac{1}{a} \cdot \sigma_{b}\right)^{2}+2 \cdot \frac{D_{\max }-b}{a^{2}} \cdot \frac{1}{a} \cdot \operatorname{cov}(a, b)} .
$$




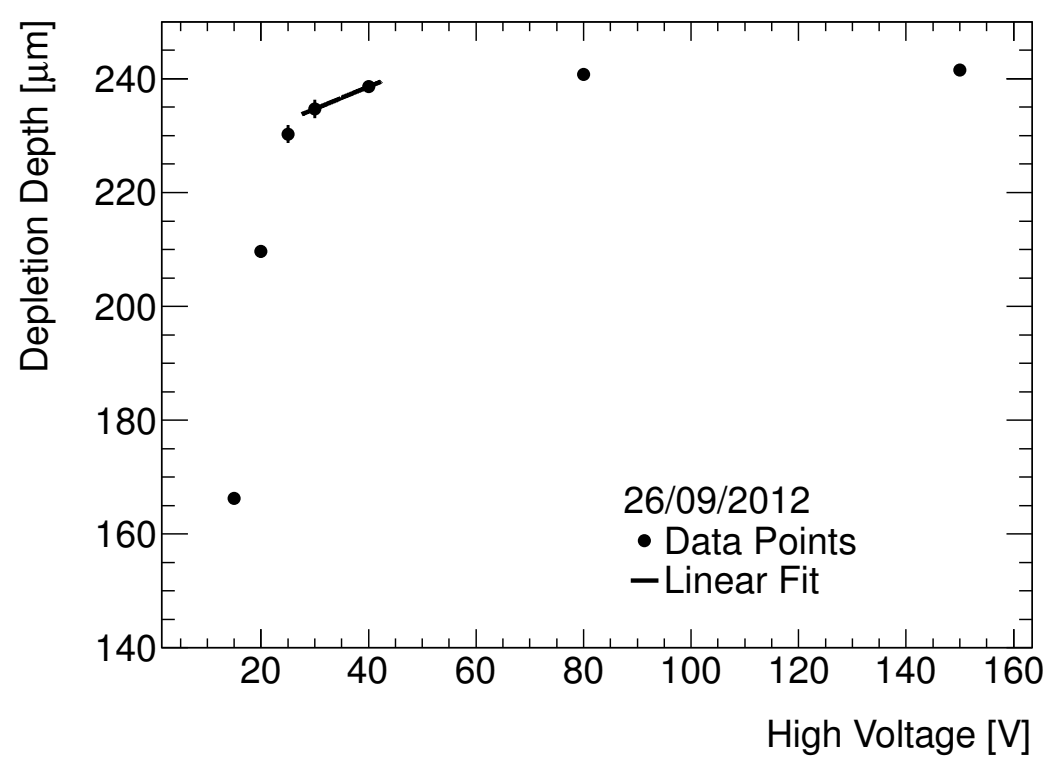

Figure 6.37.: The mean value of the calculated depletion depth is shown as a function of the bias voltage. The depletion depth scan was taken at the 26.09.2012. A linear fit is used to estimate the effective depletion voltage after type-inversion. Only the two data points closest to the expected depletion voltage value are used in the analysis.

\subsubsection{Results}

The combined results for the effective depletion voltage measurement before and after typeinversion are shown in Figure 6.38. A detailed view of the results only after type-inversion is also shown in A.5.

The results before type-inversion are illustrated as the black data points. For a detailed discussion of these results see Section 6.4. The effective depletion voltage measured after typeinversion is added in the form of the red data points and the dashed line is the model prediction.

The time difference between the last cross-talk scan and the first depletion depth scan is only four days. It is clearly visible that the measurement of the effective depletion voltage after typeinversion is larger than the measurement using the cross-talk scans. However, only a subset of the modules are measured in both scans, since the detector was in the process of undergoing type-inversion at the time.

The last cross-talk scan can only measure the modules that are not type-inverted and thus have in general a lower effective depletion voltage than the average. Only modules that have finished the process of type-inversion are measured in the depletion depth scan, thus, most modules that are included in this measurement have a higher effective depletion voltage than the average.

The measured effective depletion voltage after type-inversion is in general larger than the model prediction. First, the effective depletion voltage is in general overestimated by the linear fit. Second, as mentioned in Section 6.4 the model prediction is based on the delivered luminosity profile, the fluence expectation (FLUKA simulations) and the detector temperature. Uncertainties in the measurements of these values or simplified assumptions in the calculation 
can easily lead to a slight discrepancy between measurements and model prediction. The overall discrepancy between the model prediction and data results is still small after a measurement period of about one and a half years, especially if all uncertainties are taken into account.

Effects of beneficial annealing after cooling stoppages are visible in the model and in the measurement. The effect of the annealing in the model prediction is much larger after-type inversion, while it is similar for both measurements.

The failure of the cross-talk scans only indicates type-inversion in the pixel sensors. With the depletion depth scans it was possible to confirm this hypothesis. In addition, it is possible to monitor the evolution of radiation damage on a regular basis and evaluate the impact on the detector performance.

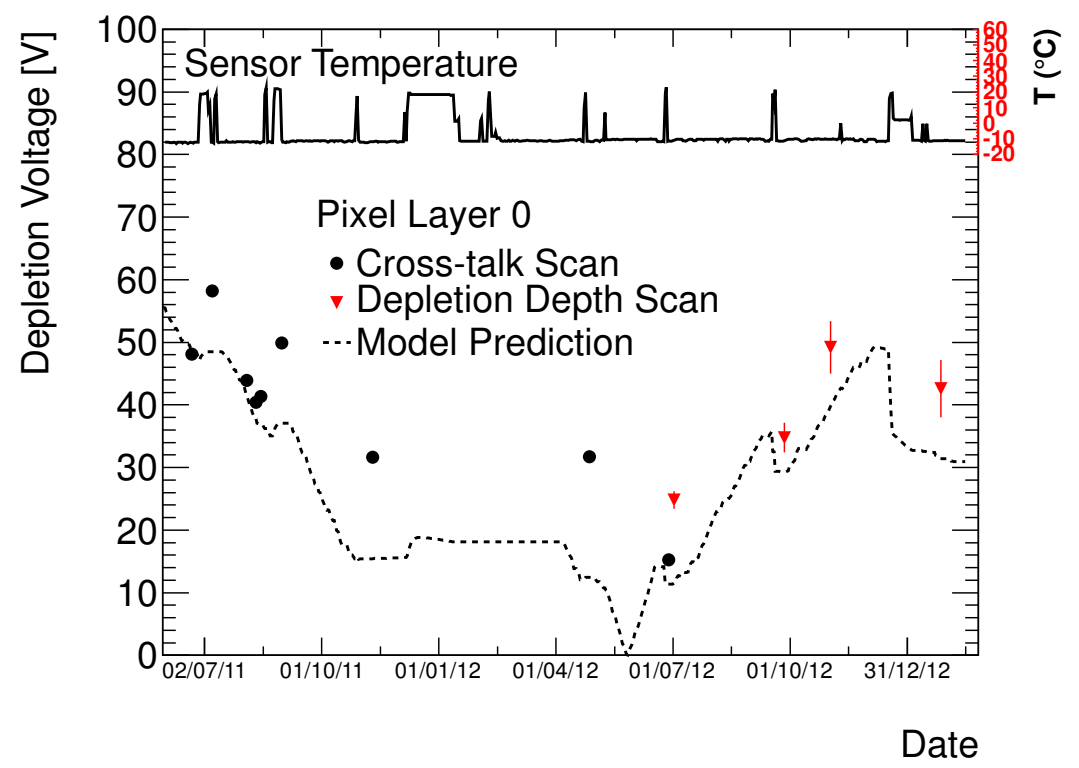

Figure 6.38.: Depletion voltage of the pixel detector as a function of time before and after typeinversion. Depletion voltage values before type-inversion have been measured with the cross-talk method. The sensor temperature is added at the top of the figure. The dashed line is a model prediction. 


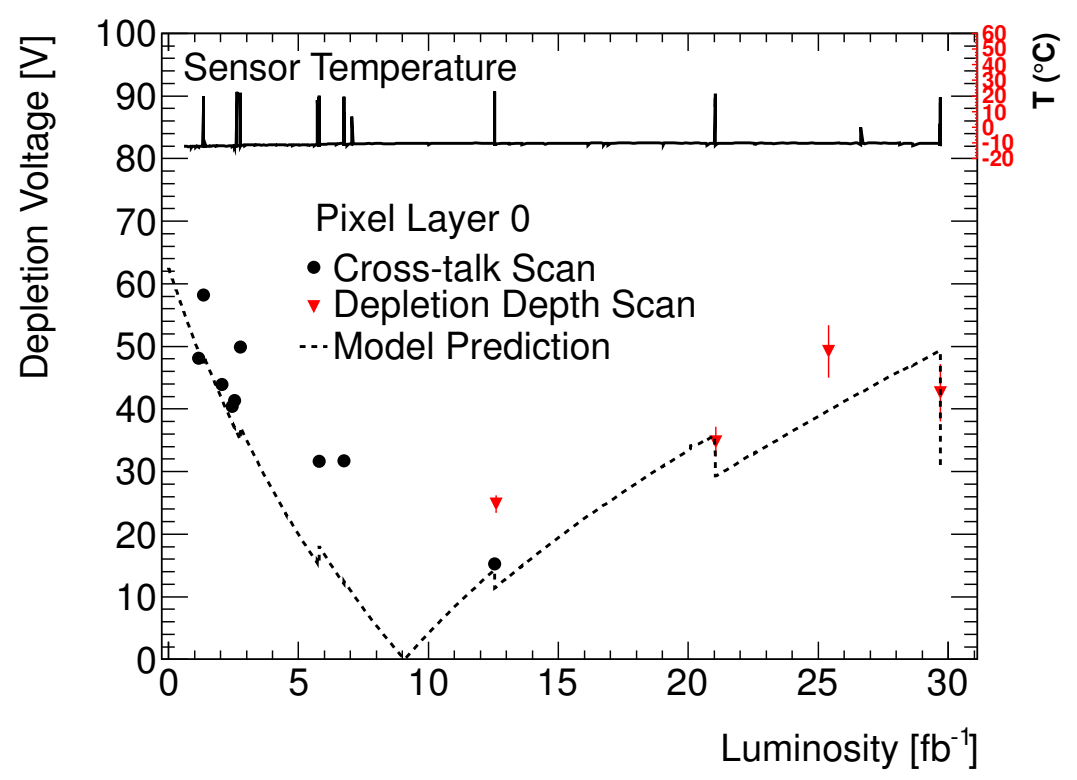

Figure 6.39.: Depletion voltage as a function of delivered luminosity. Sensor temperature and model prediction are added. The amount of delivered luminosity is very small in the lead-proton run in 2013. 


\section{Impact of Module Defects on the Detector Performance}

\subsection{Introduction}

Various actions are taken in order to counteract the impact of radiation damage and module failures as it is explained in Chapter 5. As mentioned, one of these measures is the installation of the nSQPs. The analysis presented in this chapter mainly focuses on the importance of module recovery and thus on the impact of the nSQPs on the detector efficiency. In addition to this, the impact of module failures in IBL is simulated and studied.

The module failure studies were performed initially at the beginning of 2013 in order to assess if the impact of module repairs is large enough to justify the installation of the nSQPs. In order to deploy the nSQPs the pixel detector needed to be extracted from the ATLAS detector and brought to the surface, which is a delicate task. The decision was made to install the nSQPs so the pixel detector has been extracted, the nSQPs are connected and they have been tested. During this time the presented analysis was updated with the newest information available.

The evolution of the module failures is described in Section 7.2, while the prediction of module failures in 2019 is explained in Section 7.3. A short summary of the used b-tagging algorithm is given in Section 7.4 and the used track selection is explained in Section 7.5. Finally, the impact on the detector performance of module failures is presented in Section 7.6 .

\subsection{Evolution of Module Failures}

In total 88 modules were deactivated or inefficient at the beginning of the long shutdown LS1. 79 of them were deactivated, while 2 of them were inefficient and a complete optoboard in layer 0 failed electrically, adding another 6 non-working modules. Table 7.1 shows the failures per detector layer. The largest fraction of dead modules is found in layer 2 even though the impact of radiation damage is smallest in this layer.

The module failure trend between 2008 and 2013 is presented in Figure 7.1(a), The total trend of module failures increases at a linear rate. The amount of disabled modules in layer 1 and 2 and in the disks remains relatively constant, while the number of dead modules in layer 2 increases continuously.

Figure 7.1(b) shows the trend of module failures separated into possibly repairable and possibly not repairable modules. The separation was made by the expected location of the corresponding defect. In non repairable modules, defects are located in unreachable regions (e.g. flex-hybrid), while repairable defects are located in areas that can be reached without disassembling the pixel detector, such as the optoboards. Initially it was expected that about 60 to 70 modules can be repaired. In fall 2013, all nSQPs were connected to the pixel detector and the whole detector was tested. At the time of the last test, 20 modules remained dead, while 68 modules could be reactivated, fully confirming the initial expectation. It is expected that a few additional modules will fail during the transportation and reinstallation of the pixel detector. 
However, the amount of disabled modules will be significantly lower than the 88 dead modules at the beginning of 2013 .

\begin{tabular}{|l|c|c|}
\hline & Disabled modules & Fraction \\
\hline Layer 0 & 18 & $\sim 6 \%$ \\
Layer 1 & 10 & $\sim 2 \%$ \\
Layer 2 & 47 & $\sim 7 \%$ \\
Disks & 13 & $\sim 5 \%$ \\
Total & 88 & $\sim 5 \%$ \\
\hline
\end{tabular}

Table 7.1.: Total number and fraction of disabled modules at the beginning of the long shutdown in February 2013. Most modules are deactivated in layer 2.

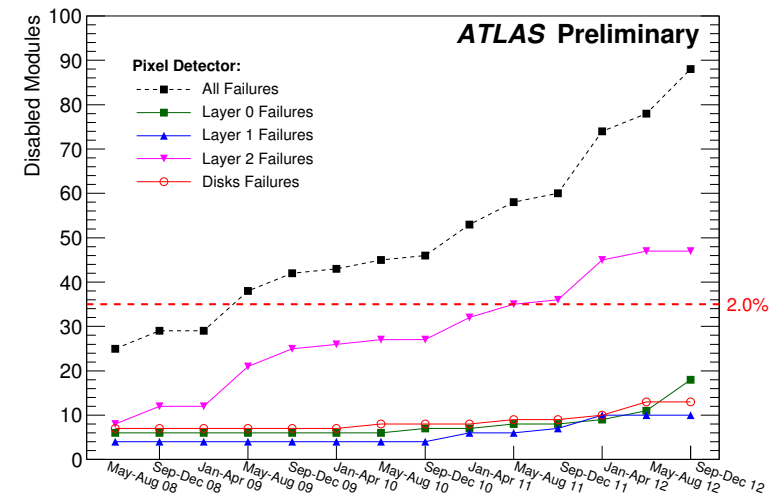

(a)

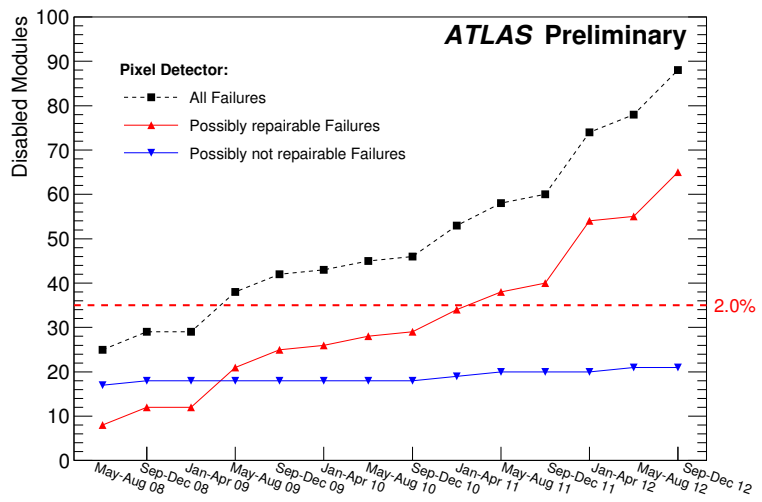

(b)

Figure 7.1.: Module failures as a function of time. The total trend and the trend per layer is shown (a). The module failures can be divided into possibly repairable and possibly not repairable failures (b) [68].

\subsection{Prediction of Module Failures}

The future performance of the detector is evaluated by predictions of module failures that are based on the observed failure trend between 2008 and 2013. Originally, two module failure trends were created. The first one without repairs and the second one including module repairs, which corresponds to the installation of the nSQPs.

Figure 7.2 shows the failure trend up to 2013 and several extrapolations. The red and the blue line show an envelope of the module failure trend without repairs. The envelope is extrapolated up to the year 2019 and indicates the amount of disabled modules in the more pessimistic case. In this case it is expected that between $6.0 \%$ and $9.6 \%$ of the pixel detector is disabled at the end of run II. Module repairs are included in the trends that are shown by the black lines. The amount of disabled modules decreases drastically after the long shutdown due to the installation of the nSQPs. Two trends after the shutdown are shown, a more pessimistic one with a module failure rate of $0.5 \%$ per year, and a more optimistic one with a failure rate of $0.2 \%$ per year. 
Only the more optimistic trend is used for the estimation of the number of dead modules in 2019 in the following analysis.

Furthermore, effects of readout limitations have been simulated. The occupancy during run I between 2009 and the beginning of 2013 was higher than initially expected. The first readout limitations have been observed in 2012 in layer 2, probably caused by the limited computing power of the RODs. Further improvements of the LHC machine will increase the luminosity in the future which will bring the system close to its bandwidth limit leading to lost or corrupted events. To address these problems, additional electrical lines were implemented into the nSQPs, increasing the readout speed for layer 1 to $160 \mathrm{Mb} / \mathrm{s}$. Due to the limited space in the cavern it was not possible to add the same improvement for layer 2. However, a new ROD system with upgraded hardware will solve the limitations in layer 2 . Thus, the bandwidth will be large enough to deal with the high luminosity in run II. However, a worst case scenario has been created for layer 1 in order to assess the impact of event loss caused by bandwidth limitations.

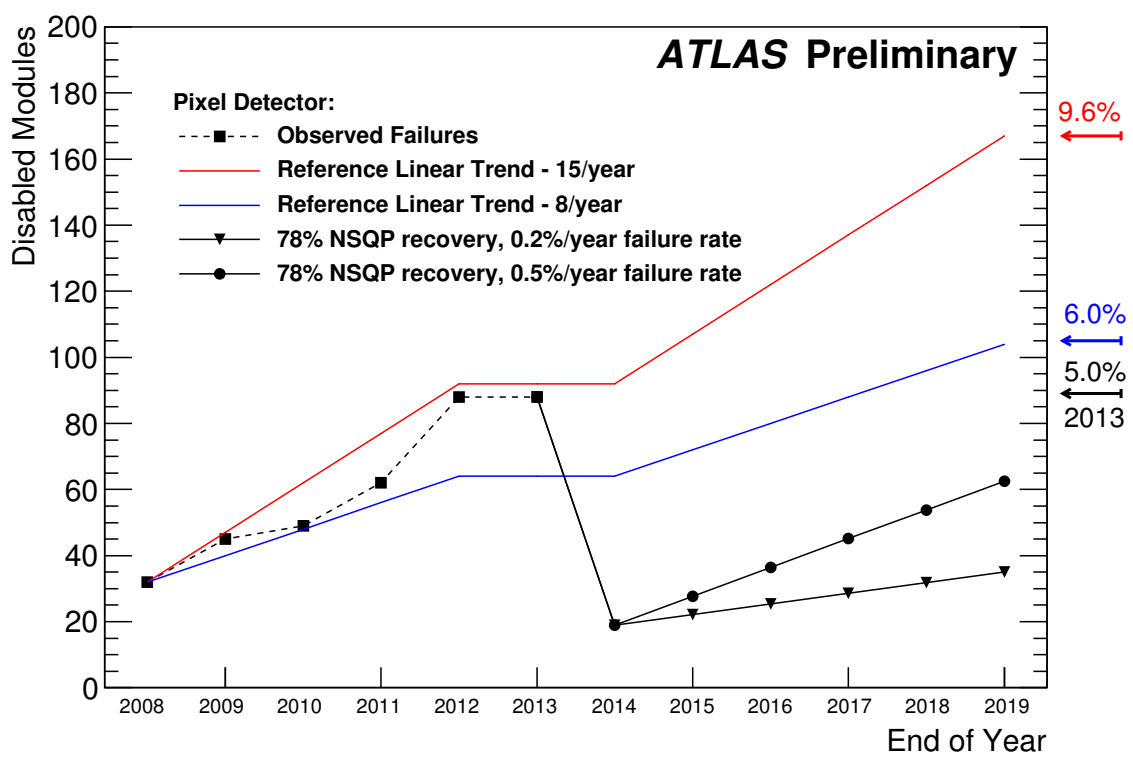

Figure 7.2.: Total trend of module failures and extrapolations. The module recovery due to the deployment of the nSQPs is taken into account. Two failure rates are shown, one with $0.2 \%$ failures per year and a second more pessimistic one with $0.5 \%$ failures per year. The red and blue lines indicate the envelope of the failure extrapolation without any module repairs 68 .

Originally all scenarios were developed in order to assess the situation between 2015 and 2019 with and without nSQPs, as explained previously. They are based on the module trends shown in Figure 7.2. However, since the nSQPs have been installed the names of the scenarios are changed in order to be able to assess the impact of module failures in a more general approach. During the update additional scenarios for different numbers of deactivated IBL modules were created, which are analysed separately.

The four scenarios for IBL are:

- Perfect IBL All IBL modules are activated.

- 5 dead IBL About $2 \%$ of the IBL modules are disabled. 
- 22 dead IBL Approximately $10 \%$ of all modules in IBL are turned off.

- 44 dead IBL Finally, about $20 \%$ of the IBL modules are deactivated.

Four different dead module scenarios have been produced for layer 0-2 and the disks as well:

- 19 dead Pix Only $\approx 1 \%$ of the pixel modules are deactivated.

- 35 dead Pix The number of deactivated modules is approximately $2 \%$.

- 88 dead Pix About 5\% of all modules in the detector are deactivated.

- 167 dead Pix In the most pessimistic scenario roughly $10 \%$ of the modules are deactivated.

Some of the scenarios are combined to assess and compare the performance of the detector at the beginning and the end of run II. The number of dead modules in layer 0-2 and in the disks corresponds to the number of deactivated modules with and without module repairs. The original scenarios created at the beginning of 2013 are summarised in the following:

- $2015+$ nSQPs IBL works perfectly and with the deployment of the nSQPs the amount of dead modules drops down to 19.

- 2015 At the beginning of the next run, the IBL is new and it is assumed that the layer works perfectly. If the nSQPs had not been installed then 88 modules would have been disabled.

- $2019+$ nSQPs + IBL Perf The most optimistic scenario. IBL works perfectly during the 4 years of operation and the reduced failure rate predicts 35 disabled pixel modules.

- 2019 + nSQPs In 20195 IBL modules are disabled - this corresponds to a failure rate of $\sim 2 \%$ after 4 years. In addition 35 pixel modules are disabled.

- 2019 Without the installation of the nSQPs 167 modules would most likely not work in layer 0-2 at the end of run II. The number of dead IBL modules is kept constant with a value of 5 .

- 2019 readfail The readout failure scenario has the same amount of dead modules as the 2019 scenario. In addition, layer 1 is completely deactivated for half of the used events. This is a worst case scenario and some mitigation can definitely be done even if the nSQPs would have not been installed.

\section{4. b-tagging}

In the following analysis a combination of the impact parameter based and secondary vertex based taggers (IP3D+SV1) has been used in order to be consistent with the IBL technical design report [43, 44]. A short summary of the b-tagging algorithms with a strong focus on the IP3D+SV1 tagger is therefore given in the following. More detailed descriptions of b-tagging in ATLAS as well as additional information on related topics such as vertex finding and jet reconstruction can be found in $[78,79$.

Normally only one hard scattering collision per bunch crossing takes place which is of interest, as mentioned in the previous chapter. The exact location of the hard scatter event (primary 
vertex position) is reconstructed precisely in the ATLAS detector [80]. However, in general several additional protons collide with each other during the same bunch-crossing creating events with a lower transverse momentum $p_{T}$. These pile-up events are created independently of the hard scattering event.

In general, b-tagging algorithms use the properties of reconstructed calorimeter jets 78,81 as well as tracks 82$]$ that can be associated to a jet. Tracks are associated to a jet if they lie within

$$
\Delta R=\sqrt{\Delta \phi^{2}+\Delta \eta^{2}}<0.4,
$$

in this study. Several cuts on the distance $\Delta R$ are possible, however a value of 0.4 is widely used.

B-tagging algorithms exploit the fact that the b-hadron tends to fly approximately a few millimetres before it decays and thus has a measurable displacement from the primary interaction vertex. Several b-tagging algorithms can be used in order to indentify b-jets. Important taggers are the secondary vertex (e.g. SV1) and impact parameter based taggers (e.g. IP3D) [78, 83] as well as the JetFitter algorithm 84, 79]. Furthermore, the MV1 tagger is a tagger that is applied widely in ATLAS. It uses the outputs of the SV1, IP3D and the JetFitterCOMBNN taggers as an input for a neural network based b-tagging algorithm [85].

\subsubsection{The Impact Parameter based Algorithm IP3D}

The IP3D tagger is a likelihood ratio based algorithm that uses two different impact parameters for the identification of b-jets. In the following, track trajectories are defined at the point of closest approach, $P_{A}$, with respect to a reference point $P_{0}$. The point $P_{0}$ is defined as the location of the primary vertex. The distance between $P_{A}$ and $P_{0}$ in the transverse plane (global $R \phi$-plane, defined in Section 3.3.1) is called $d_{0}$, while the distance parallel to the global z-axis is called $z_{0}$,79, 86.

The transverse impact parameter is simply defined as the distance $P_{A}-P_{0}$ in the $R \phi$-plane $[79$ :

$$
I P_{r \phi}=d_{0} .
$$

The distance between the point of closest approach and the primary vertex in longitudinal direction is approximated by a straight line. Thus, the longitudinal impact parameter is calculated using the opening angle $\Theta$ measured with respect to the global $z$-axis 79 :

$$
I P_{z}=z_{0} \sin (\Theta) .
$$

In order to further improve the discriminating power a lifetime sign is defined. It is positive if the track intersects the reconstructed jet axis in front of the primary vertex, which is more likely for tracks originating from a secondary vertex. The sign is negative if the corresponding track intersects the jet axis behind the vertex opposite to the jet direction. An example for a positive and a negative lifetime sign is shown in Figure 7.3 .

The formula for the lifetime sign can be written as 79 :

$$
\operatorname{sign}_{3 D}=\operatorname{sign}\left(\left[\vec{p}_{\mathrm{trk}} \times \vec{p}_{\mathrm{jet}}\right] \cdot\left[\vec{p}_{\mathrm{trk}} \times \Delta \vec{r}_{I P}\right]\right),
$$

where $\vec{p}_{\text {jet }}$ is the jet momentum measured in the calorimeter and $\vec{p}_{\text {trk }}$ is the track momentum at the point of closest approach. $\Delta \vec{r}_{I P}$ is the difference between the position of the primary vertex and the point of closest approach of the selected track $\vec{X}_{P V}-\vec{X}_{I P}$. The IP3D tagger uses the 


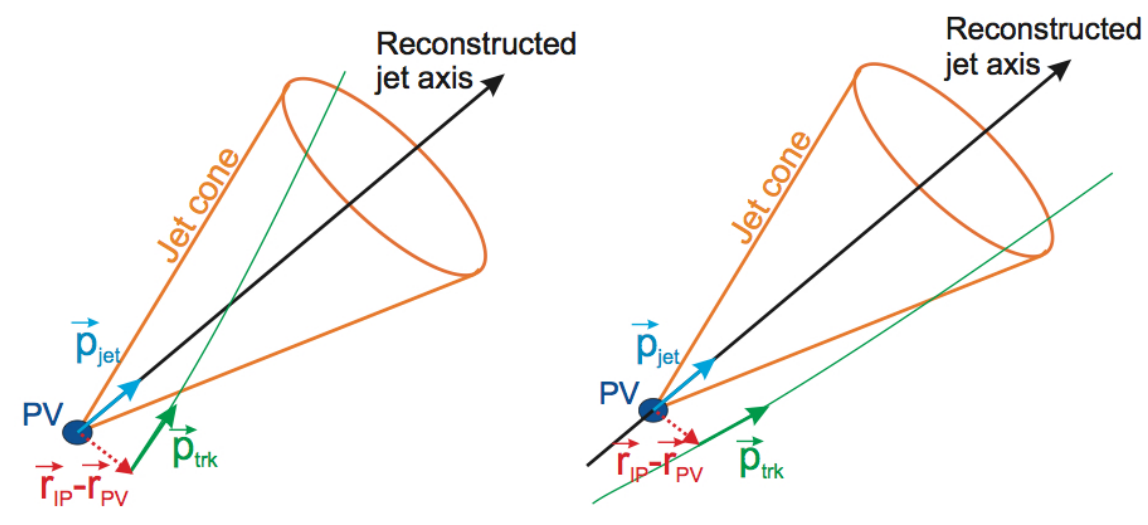

Figure 7.3.: Schematic view of a jet and a selected track. A track with a positive lifetime sign is shown on the left, while the track on the right has a negative lifetime sign [79].

two previously mentioned parameters, however, the estimation of the discriminator is similar for several methods. For the sake of simplicity the measurement is first explained for a generic impact parameter IP, while the application for IP3D is described afterwards.

The lifetime sign and the impact parameter are used to calculate the impact parameter significances

$$
S_{i}=\operatorname{sign}_{\mathrm{r} \phi} \cdot \frac{I P_{i}}{\sigma_{I P_{i}}}
$$

for each track $i$ associated to the jet. For the IP3D tagger two significances are calculated, one with $I P_{i}=I P_{r \phi}$ and the other one with $I P_{i}=I P_{z}$. After they are calculated they are then compared to normalised reference distributions for the b-jet hypothesis $b\left(S_{i}\right)$ and for the light jet hypothesis $u\left(S_{i}\right)$, which are derived from Monte Carlo samples (see Figure 7.4). The ratio of the reference distributions describes the weight which is then summed up for all tracks associated to the jet 78 :

$$
W_{\mathrm{jet}}=\sum_{i=1}^{N_{T}} \ln \frac{b\left(S_{i}\right)}{u\left(S_{i}\right)},
$$

where $N_{T}$ is the number of tracks. A jet is tagged as a b-jet if its weight is larger than the cut value on the jet weight. Its value is chosen depending on the desired b-tag efficiency and the corresponding mistag rate (see Subsection 7.4.4).

Selected tracks can be separated into different categories $j$ in order to further improve the performance of the tagger. The separation depends on their properties such as shared hits in the pixel detector. This change can be easily implemented by separating the reference distribution the same way and comparing each track to its corresponding reference distribution. This leads to [78]:

$$
W_{\mathrm{jet}}=\sum_{j=1}^{N_{C}}\left(\sum_{i=1}^{N_{T}^{j}} \ln \frac{b_{j}\left(S_{i}\right)}{u_{j}\left(S_{i}\right)}\right),
$$

where $N_{C}$ is the number of track categories and $N_{T}^{j}$ is the number of tracks per category. Several taggers use this likelihood approach with different types of parameters and reference 
distributions. The IP3D tagger uses two-dimensional reference distributions of the signed impact parameter significances. The reference distributions in Equation 7.7 thus become functions of the longitudinal and transverse impact parameters: $b_{j}\left(S_{i, r \phi}, S_{i, z}\right), u_{j}\left(S_{i, r \phi}, S_{i, z}\right)$. An example of the distributions is shown in Figure 7.4 .
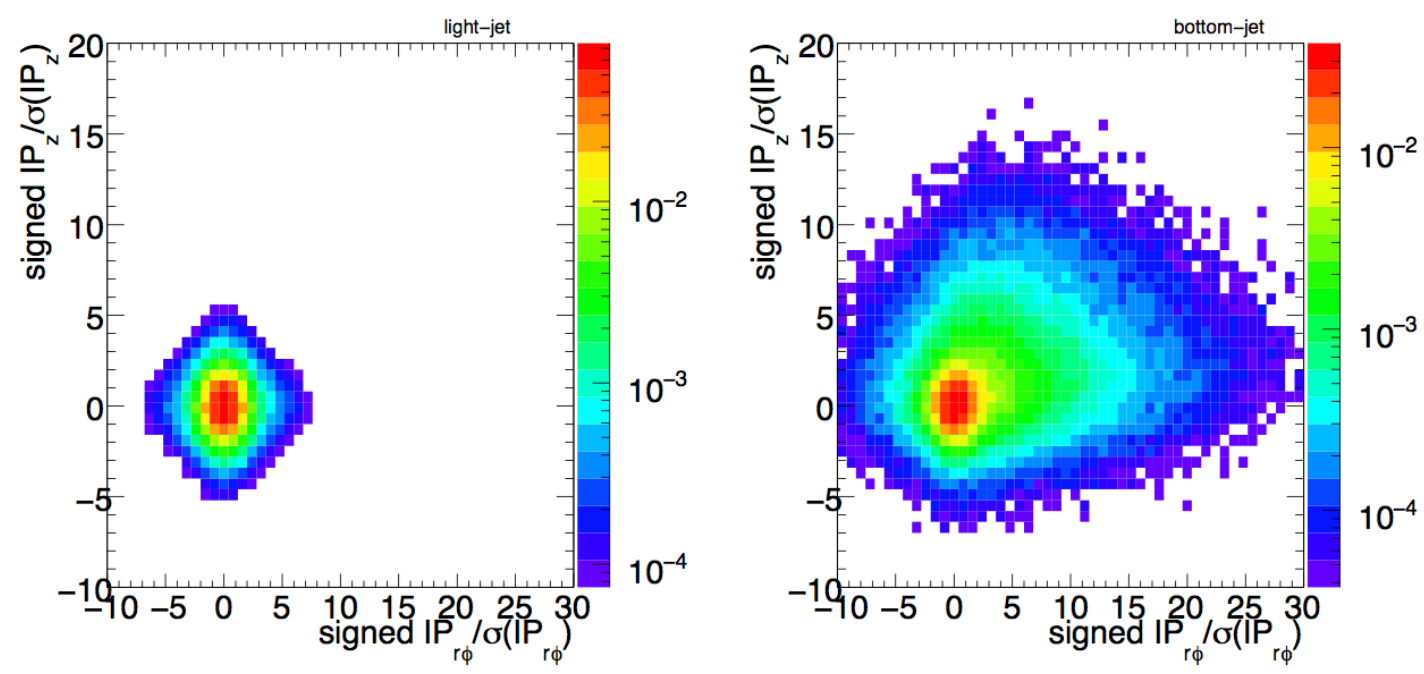

Figure 7.4.: Reference distributions as a function of the transverse and longitudinal impact parameter significances. An example distribution is shown for light jets on the left and b-jets on the right. Both distributions are logarithmic in scale and normalised 79$]$.

\subsubsection{The Secondary Vertex based Algorithm SV1}

In most cases a c-hadron is created during the decay of b-jets, which can also travel for a substantial distance before it decays and thus forms a third vertex. However, in general it is very challenging to separate the secondary vertex from the third c-decay vertex due to resolution constraints in the detector. The secondary vertex based algorithms approximate the vertex position by reconstructing an inclusive vertex of the $b$ and $c$ hadron decay.

The search for the vertex is started by taking the tracks in the jet and combining them in two track vertices [78]. Only tracks that originate far enough from the primary vertex are regarded. Furthermore, reconstructed two track vertices that originate from $K_{S}^{0}, \Lambda^{0}$ and photon decays are removed using their reconstructed vertex mass. An iterative approach is then used on all tracks that remain in order to find the secondary vertex. The track that contributes the most to the resulting $\chi^{2}$ is removed until a good result is found. The SV1 tagger then relies on two reference distributions that take advantage of the secondary vertex properties. One two dimensional histogram uses the invariant mass of all tracks associated to the secondary vertex and the track energy ratio, which is defined in the following. For the calculation of the track energy ratio the energies of the tracks associated to the second vertex and the energies of all tracks are summed up. The ratio of these two sums is the track energy ratio. The one dimensional distribution uses the number of two track vertices. The jet weight is then calculated in a similar way as it is done in Equation 7.7 . 


\subsubsection{Combination of Algorithms}

The IP3D and SV1 algorithms are combined by simply adding the two calculated event weights. The sum is then used as the new discriminator. This treatment is only correct under the assumption that the likelihood ratios calculated by both algorithms are uncorrelated. However, a large improvement of the performance is observed for the combined tagger IP3D+SV1, even if this assumption is not correct 79 .

\subsubsection{Efficiency and Jet Rejection}

The flavour $q$ of jets in Monte Carlo simulations is defined by the heaviest quark that is generated in its vicinity. For example a jet is defined as a b-jet $(q=b)$ if a b-quark was generated within a distance of $\Delta R<0.3$ in jet direction. The efficiency of tagging a jet of flavour $q$ as a b-jet is 79 :

$$
\epsilon_{q}=\frac{\text { Number of q-jets tagged as b-jets }}{\text { Number of q-jets }} .
$$

If $q=b$, then the above described formula for the b-jet efficiency is in general used. However, if the true flavour $q$ of a jet is different to the flavour $b$ (e.g. light jets), then the jet rejection $r=\frac{1}{\epsilon_{q}}$ is often used as a measure for the impact of mistagging a jet of a different flavour as a b-jet.

\subsection{Data Samples and Selection}

Two $t \bar{t}$ samples from the MC12 production are chosen in the analysis: one with no pile-up and one with a pile-up of $\langle\mu\rangle=60$. The centre of mass energy of the chosen samples is $\sqrt{s}=14 \mathrm{TeV}$. The version of the software package athena used for the reconstruction and the analysis of the Monte Carlo samples is 17.3.10.1.2,IBLProd and the version of the geometric model used in the simulation is ATLAS-IBL-03-00-00. The mentioned geometric model description for IBL is only equipped with modules that use planar silicon sensors. In addition, the change in the material distribution at high $\eta$ values due to the installation of the nSQPs was not implemented at the time. In the current analysis the neural network pixel clustering is not used, because it had not been adapted for the track reconstruction with 4 pixel detector layers.

Several dead module maps were created and then used in the reconstruction phase in order to deactivate the correct number of modules according to the scenarios described in Section 7.3. The scenario that simulates readout failures is a combination of two scenarios. Half of the events in this scenario were taken from the standard 2019 scenario, while for the other half of the events layer 1 was completely masked during reconstruction.

Only jets with a pseudorapidity of $|\eta|<2.5$ and a jet momentum of $p_{T}>20 \mathrm{GeV}$ have been defined as taggable jets and thus are considered in the following analysis. In pile-up Monte Carlo samples an additional Jet Vertex Fraction $(J V F)$ cut is introduced in order to reduce the amount of pile-up jets. The JVF value for the jet $i$ and the vertex $j$ is defined as 87]:

$$
J V F\left(\text { jet }_{i}, \operatorname{vtx}_{j}\right)=\frac{\sum_{k} p_{T}\left(\operatorname{trk}_{k}^{\text {jet }_{i}}, \operatorname{vtx}_{j}\right)}{\sum_{n} \sum_{l} p_{T}\left(\operatorname{trk}_{l}^{\text {jet }_{i}}, \operatorname{vtx}_{n}\right)} .
$$

The general idea of the JVF is also illustrated in Figure 7.5(a) showing two jets with associated tracks as an example. During the calculation only tracks associated to vertex candidates are used and the JVF values reach from 0 to 1 , while jets with no tracks associated have a value of -1 . 
If the primary vertex is taken as a reference, then the value of a jet is 0 if all tracks originate from vertex candidates that were not selected as the primary vertex. It is 1 if all tracks are associated to the primary vertex, while values in between indicate a mixture of associated tracks. Examples of the JVF distribution for pile-up and hard scatter jets are shown in Figure 7.5(b). The shown distributions were calculated using $\mathrm{Z} \rightarrow$ ee + Jets Monte Carlo samples. The JVF value is a very good indicator for pile-up interactions, since vertex candidates not selected as the primary vertex are most likely created by low $p_{T}$ interactions. In the following analysis only jets with a $J V F>|0.5|$ are considered.

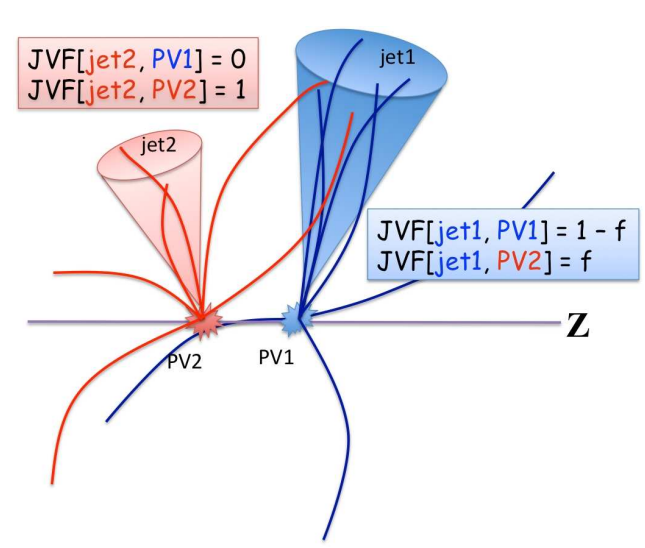

(a)

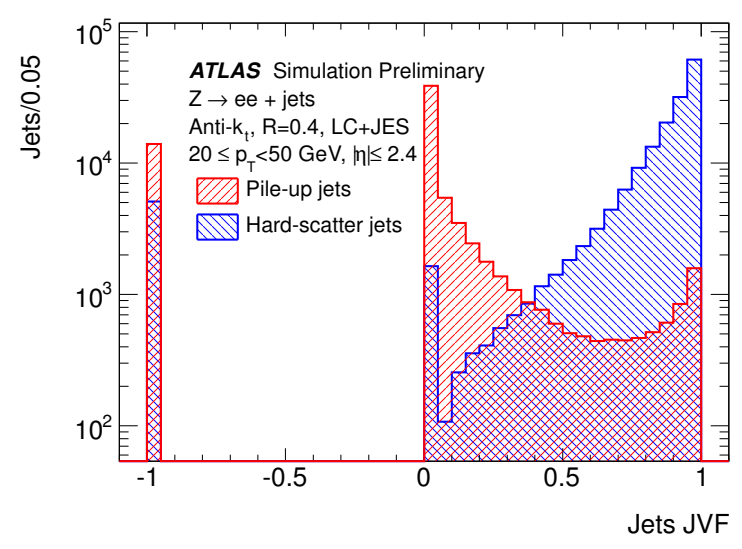

(b)

Figure 7.5.: A schematic view of two jets demonstrating the principle behind jet vertex fraction (a) [87]. Example plot of the JVF, calculated for simulated $\mathrm{Z} \rightarrow$ ee + Jets events (b) [87].

\subsection{Impact on Light Jet Rejection}

\section{Jet Rejection with pile-up}

In order to assess the impact of the module failures the light jet rejection as a function of the b-jet efficency is evaluated in this study. Dead modules of course lead to a decrease of the active area in the detector and thus eventually cause a drop in the tracking resolution. These effects have an impact on the ability to separate b-jets from light jets.

A harder cut on the jet weight naturally increases the light jet rejection and thus decreases the background, while also decreasing the b-jet efficiency. The light jet rejection as a function of the b-jet efficiency is shown in Figure 7.6 for several scenarios. The jet rejection plot is only shown as an example, because this study focuses on the difference in detector performance, which is best visible in jet rejection ratio plots.

Figure 7.7 shows the jet rejection ratio for a more detailed analysis. In the ratio plot the scenario "5 dead IBL +35 Pix" is used as the reference model. As is clearly visible, the deactivation of 5 IBL modules reduces the light jet rejection by about $10 \%$ to $20 \%$. The difference for a detector with and without the nSQP package is expected to be about 132 dead modules. The change in light-jet rejection between these two scenarios is the difference between the "5 dead IBL + 35 Pix" and the "5 dead IBL + 167 Pix" scenario. As is clearly visible, the 


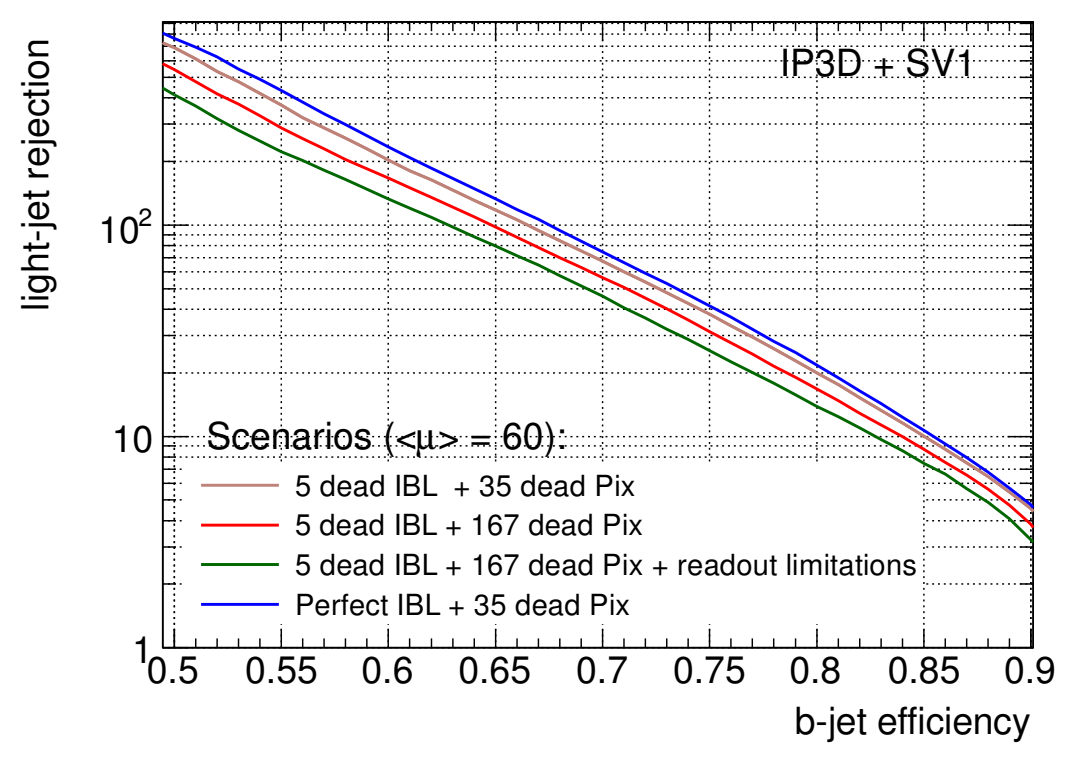

Figure 7.6.: Light jet rejection as a function of the b-jet efficiency. Five different scenarios are shown. The pile-up in this example is $\langle\mu\rangle=60$.

difference is about $20 \%$. The pessimistic worst case scenario decreases the light-jet rejection by $30 \%-40 \%$.

All scenarios shown in Figure 7.7 are scenarios for 2019 at the end of run II (see Section 7.3). As explained, it is expected that 35 pixel modules are disabled at the end of run II with nSQPs installed, while 167 would have been disabled without module repairs. The decision was made in 2013 to install the nSQPs, due to the fact that the impact of module repairs on the detector performance is clearly visible. In addition, it is obvious that the worst case scenario needs to be avoided, therefore, actions have been taken to increase the bandwidth and computing power, which will prevent these problems. Due to these activities it is currently expected that less modules are deactivated in 2019 than at the beginning of 2013, which will ensure a good performance of the detector throughout run II.

The detector model for IBL and the reconstruction software is not final and the b-tagging algorithms are not yet optimised for a 4 layer detector, as mentioned. These effects will have a big impact on the total jet rejection, while the impact on the ratio is expected to be small.

In addition, the impact of module failures in the IBL is studied independently. The change in jet rejection ratio as a function of the number of deactivated IBL modules is shown in Figure 7.8. The " 5 dead IBL" scenario with 35 deactived modules in the outer layers is again taken as the reference. It is obvious that the impact of dead IBL modules is large, due to the fact that the new detector layer is very close to the interaction point. Thus, one IBL module covers a larger angular fraction than one outer pixel module. The deactivation of 5 modules already changes the ratio visibly, while the reduction for 22 deactivated IBL modules lies between $20 \%$ and $40 \%$ with respect to "5 dead IBL". This is larger than the impact of deactivating 132 outer layer modules. The deactivation of approximately $20 \%$ of IBL decreases the performance further, however, the increase in the degradation is not linear and thus not as big as it is for $10 \%$. 


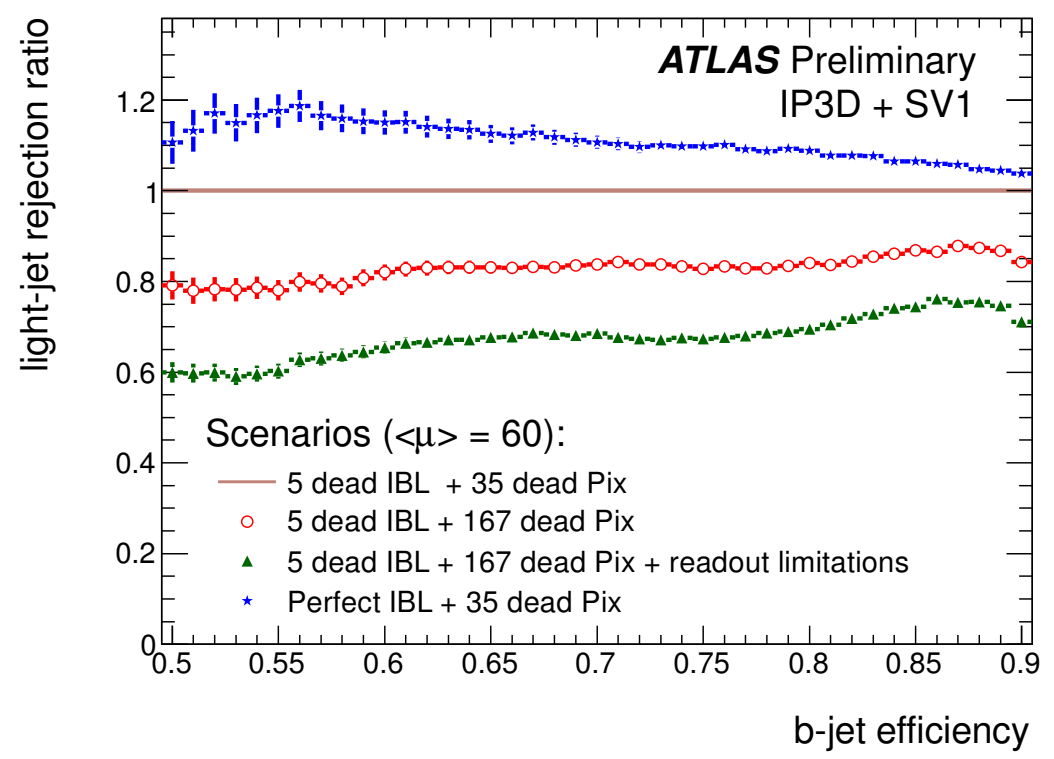

Figure 7.7.: Light jet rejection ratio with respect to the standard scenario as a function of the b-jet efficiency for pile-up $\langle\mu\rangle=60$. Various scenarios for 2019 are compared [68].

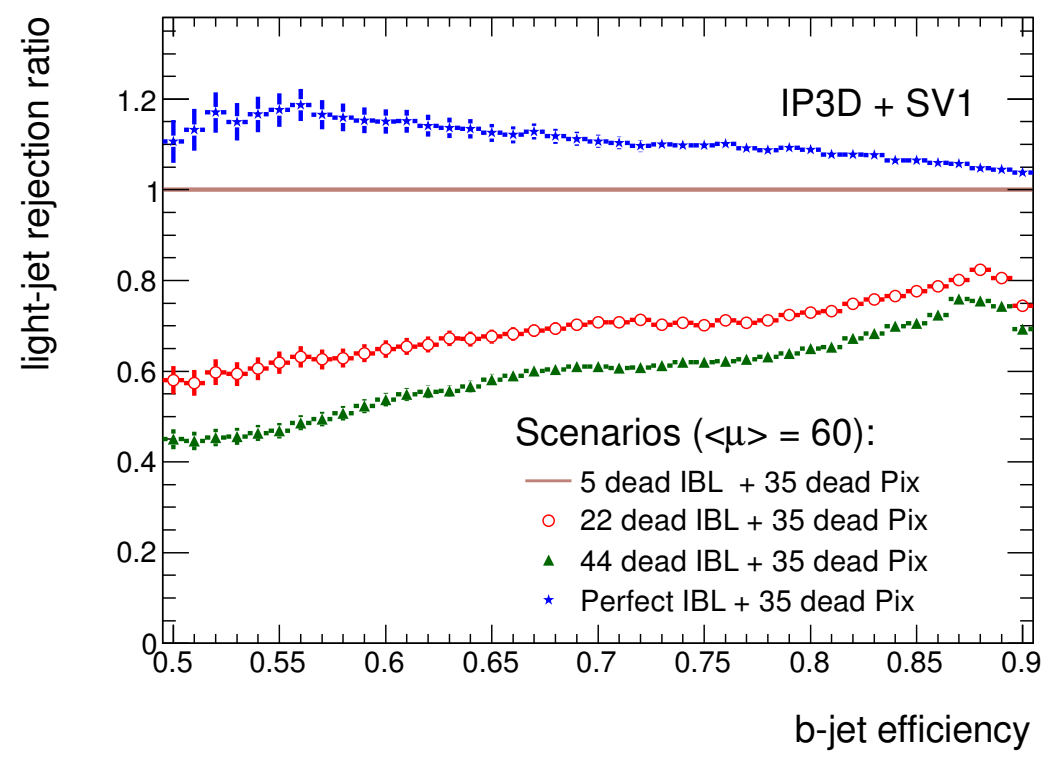

Figure 7.8.: Light jet rejection ratio with respect to the standard scenario is shown. It is a function of the b-jet efficiency and the pile-up of the simulated sample is $\langle\mu\rangle=60$. Different scenarios for the IBL are shown. 


\section{Jet Rejection without pile-up}

Studies without pile-up are performed in order to be able to compare the impact of pile-up on the jet-rejection studies. Figure 7.9 shows the light jet-rejection for two different comparisons of no pile-up scenarios. Figure 7.7 and Figure $7.9(\mathrm{a})$ show the same scenarios and the only difference is the number of pile-up events in the corresponding Monte Carlo samples. The total jet rejection ratio differs slightly for the different scenarios, but in general it is very similar. The impact of pile-up on the total jet rejection ratio in this case is small. The impact on the total jet rejection is of course large, since pile-up events in general consist of a large amount of light jets.

The impact of dead pixel modules in the outer layers can bee seen in Figure 7.9(b), Between 19 and 167 modules are disabled. The reference scenario in this plot has changed, it is the scenario with all IBL modules activated and only 19 pixel modules deactivated. This scenario is expected at the start of run II, since all repairable modules are reactivated and the IBL is a new detector layer. In this case only the impact of module failures in the outer layers is studied and thus the IBL stays completely activated in all scenarios. 35 dead modules are expected in 2019 after module repairs. The impact on the performance is relatively small compared to the scenarios without module repairs (88 and 167 dead modules), due to the reduced failure rate, again showing the importance of the nSQPs.

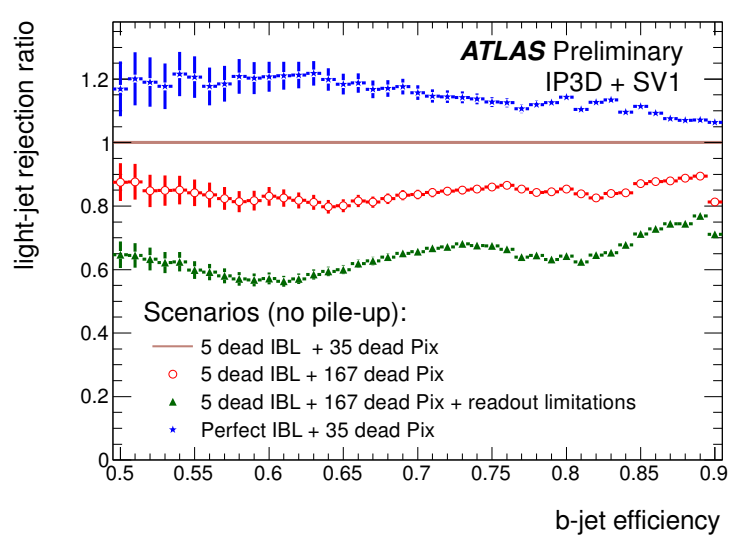

(a)

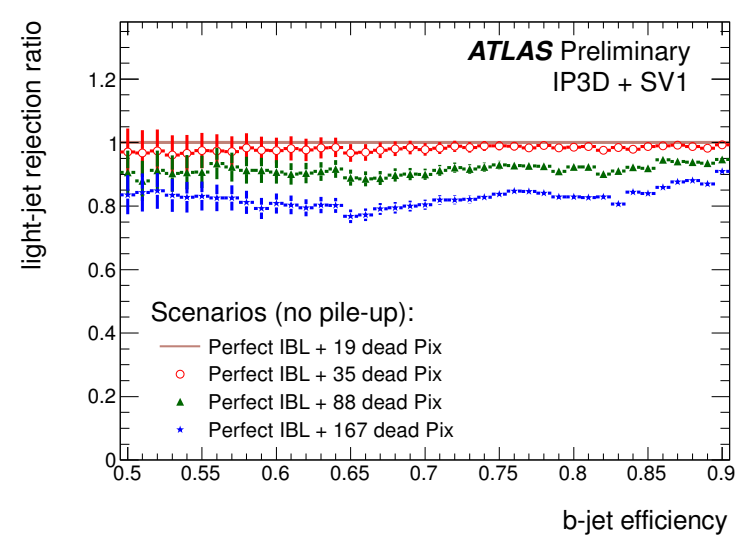

(b)

Figure 7.9.: Light jet rejection ratio shown as a function of the b-jet efficiency. No pile-up is present in the used Monte Carlo simulations. A comparison for different scenarios in 2019 (a) and only the impact of dead modules in layer 0-2 (b) is shown 68. 


\section{Conclusion and Outlook}

In the course of this thesis, fully data driven measurements for the monitoring of radiation damage have been developed. This makes it possible to measure the depletion depth without partially relying on Monte Carlo data as was necessary for earlier measurements. It is now possible to measure the effective depletion voltage before and after type-inversion as well as the depletion depth after type-inversion.

The effective depletion voltage was measured before type-inversion using cross-talk scans that were taken regularly. It decreased with time up to the point of type-inversion as expected. All measurements have been compared to a model prediction. The agreement is in general good, however measurements taken shortly after cooling stoppages showed a much larger impact of beneficial annealing than it was expected from the model prediction. The cross-talk scans revealed that the modules in layer 0 were close to type-inversion in summer 2012, while layer 1 was expected to undergo type-inversion in fall 2012.

In order to confirm that type-inversion has occurred and to further monitor the evolution of radiation damage, track based depletion depth scans replaced the cross-talk measurements. The depletion depth measurements have been tested with fully depleted sensors and the results are in agreement with the sensor thickness. The first scans have shown clearly that the depleted region grows now from the backside in layer 0 and layer 1 , thus confirming the occurrence of type-inversion. Further depletion depth scans revealed a decrease in the depletion depth for a fixed bias voltage. This dependence was used to extract the effective depletion voltage after type-inversion. Results were again compared to the model prediction showing a similar trend, however, the measured effective depletion voltage is slightly larger than the model predicts it.

The direct measurement of radiation damage and type-inversion in a silicon detector that is in operation is in contrast to standard laboratory measurements, which use fixed environmental conditions. Type-inversion took place in the time window that was predicted by the model descriptions even though some deviations from the model prediction are observed, confirming that the impact of radiation damage on pixel detectors can be predicted reliably for years in advance.

The measurement techniques developed in this thesis can be used to further monitor the evolution of radiation damage, which is a very important task in order to ensure that the performance of the detector is met until the designated lifetime of the sensor is reached. Furthermore it is possible to use these measurements to improve the simulation of the ATLAS detector. In earlier versions of the digitisation package no radiation damage effects were simulated. However, the impact of radiation damage is being added since it will become more severe with time. The results of the radiation damage measurements presented in this thesis can be used to estimate the effective doping concentration in the sensor which can be fed back into the simulation of radiation damage in order to improve it. In addition, the observation of the annealing phenomenon could be used to improve future model predictions of the impact of radiation damage in the pixel detector.

In the future, it would be possible to add a few improvements to the depletion depth measurements. The estimation of the cluster depth could be improved, since it is susceptible to the systematic shift of the Landau distribution. Changing the measurement could increase its 
precision further. In addition, the largest systematic uncertainty is the charge collection uncertainty in the Front-End chips. Further studies of this uncertainty might reveal a more precise dependence of the amount of collected charge and its deviation. This additional information could increase the precision of the measurement further.

The impact of module failures on the detector has been studied and it was decided to install the nSQP system during the long shutdown in 2013/2014. This decision was partially based on the results of the module failure studies presented in this thesis, since its benefit outweighs the risks of the installation procedure. This installation was completed during LS1 2013/2014.

The module failure studies have been updated after the decision was made and they are now used as a general outline for the impact of module failures over time. Overall the pixel detector is in good condition, since many modules could be reactivated and the impact of the radiation damage in the sensor is in the same order of magnitude as it was expected during the construction of the detector. 


\section{A. Additional Radiation Damage Figures}

\section{A.1. Additional Depletion Voltage Plots}

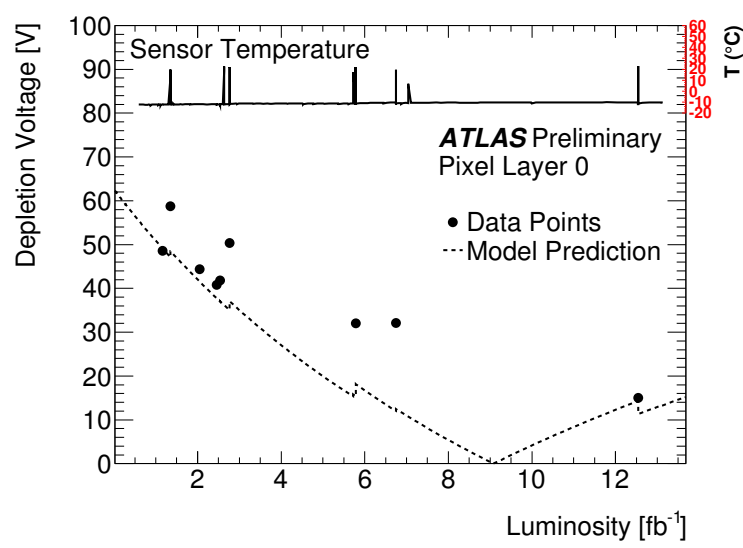

(a)

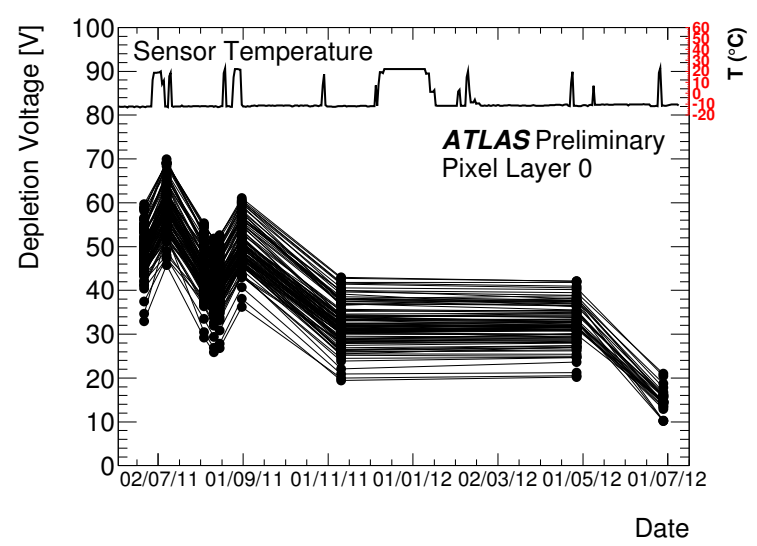

(b)

Figure A.1.: Depletion voltage vs delivered luminosity is shown. Each point represents the measured depletion voltage for the b-layer (a). Depletion voltage vs date is shown for each module in the b-layer (b) 68 .

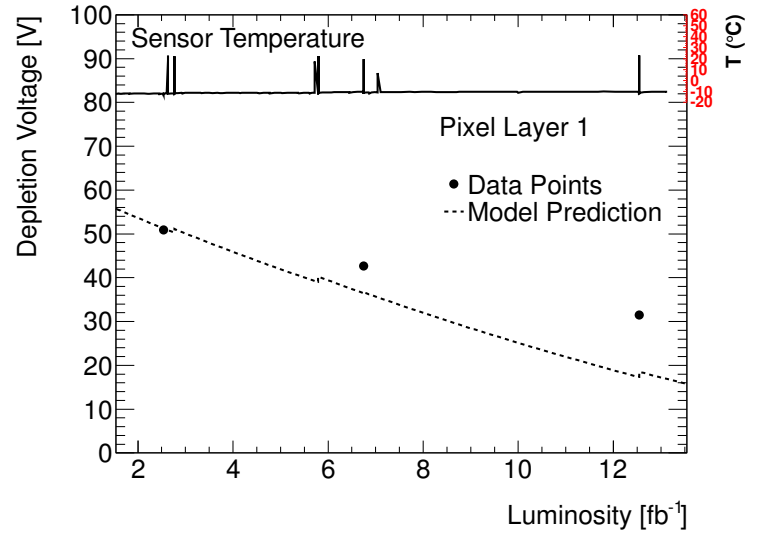

(a)

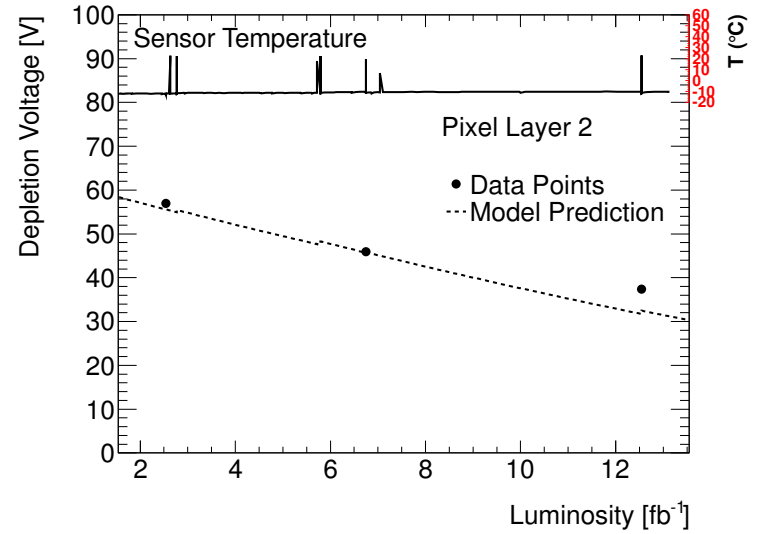

(b)

Figure A.2.: Depletion voltage as a function of the total delivered luminosity shown for layer 1 (a) and layer 2 (b). 


\section{A.2. Additional Cluster Depth Plots}

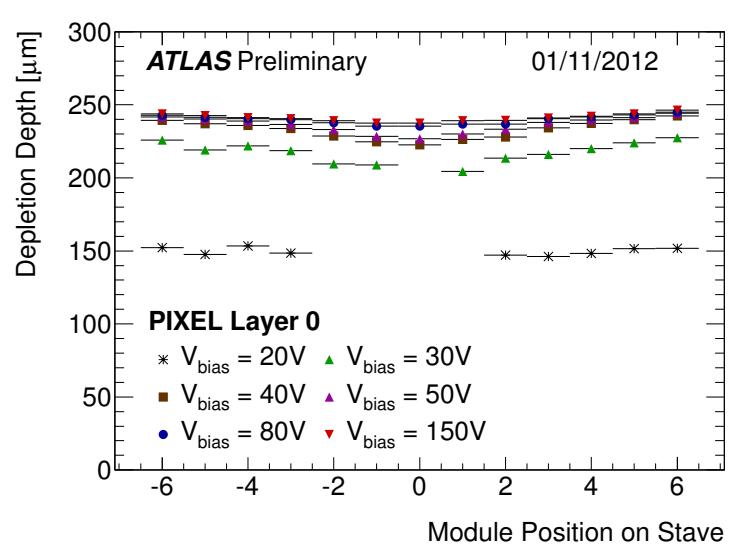

(a)

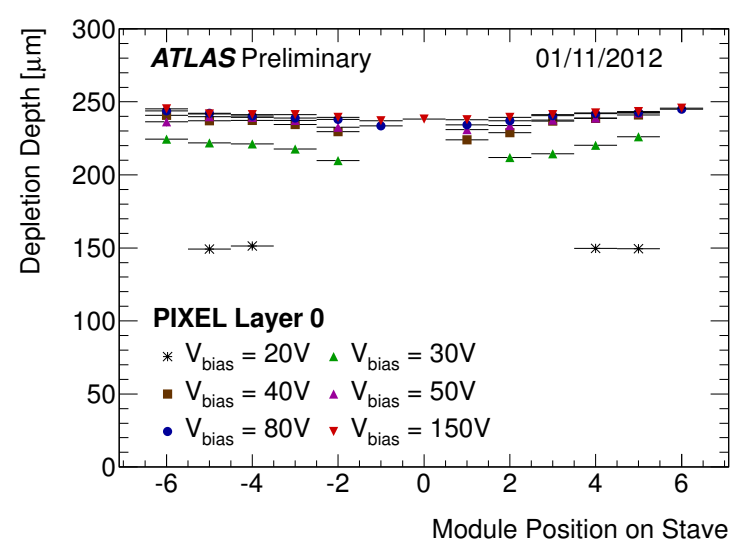

(b)

Figure A.3.: Depletion depth as a function of the stave position in the first pixel layer. The bias voltage scan has been taken at the 01.11.2012. Result are produced using reconstructed tracks from the muon stream(a) and the minbias stream(b) [68].

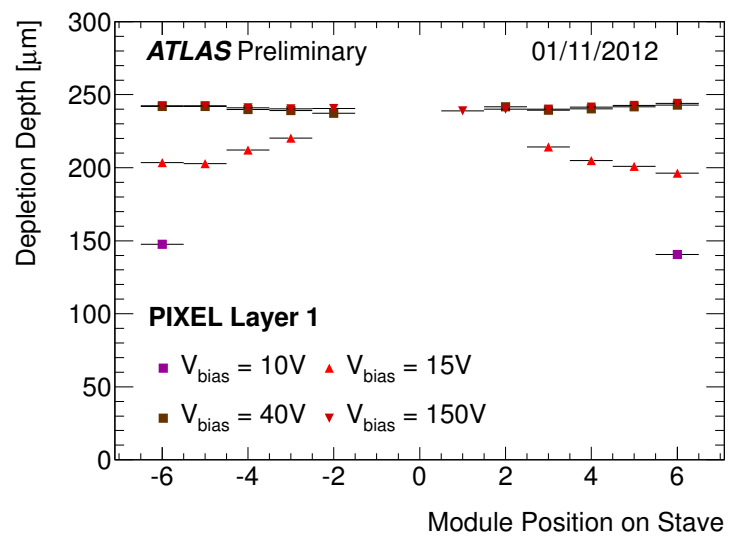

(a)

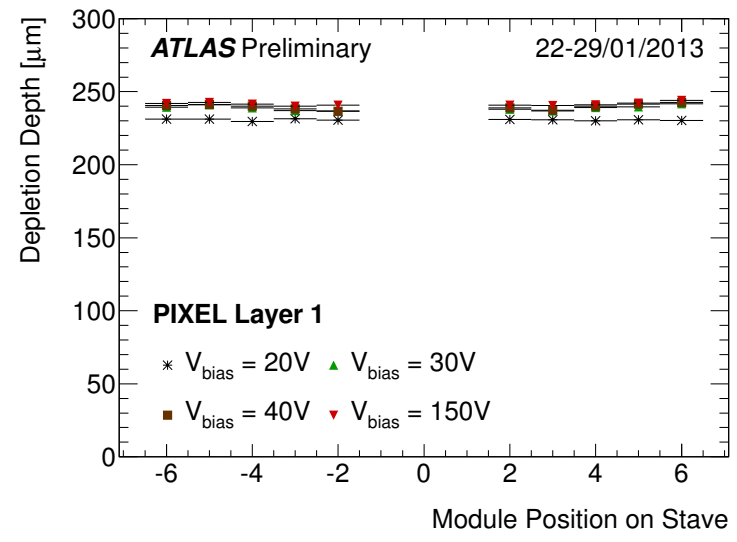

(b)

Figure A.4.: Depletion depth shown as a function of the module position on stave. Layer 1 is shown [68]. 


\section{A.3. Additional Depletion Voltage Plots after type-inversion}

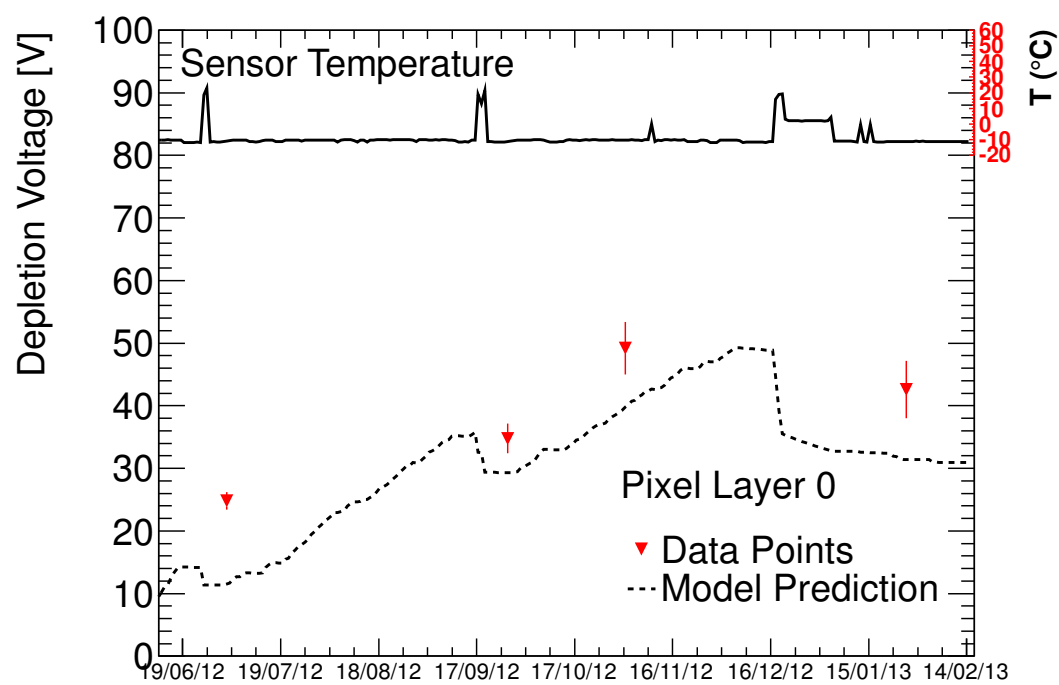

Date

(a)

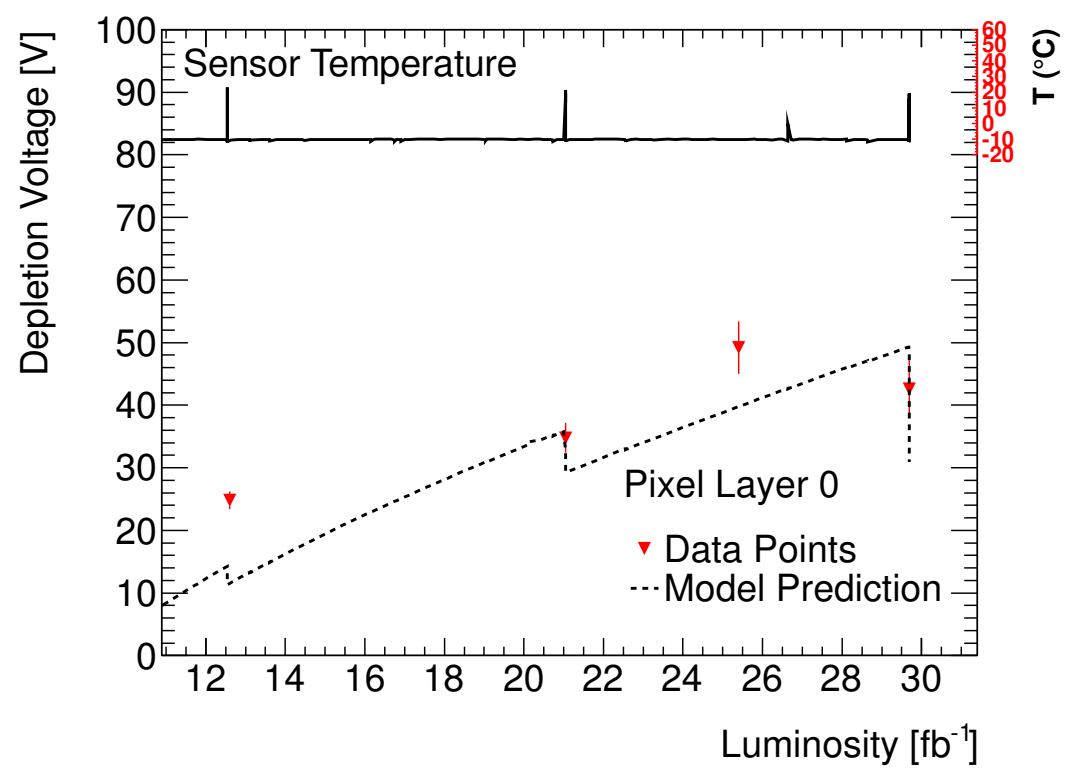

(b)

Figure A.5.: Depletion voltage of the pixel detector as a function of time (a) and delivered luminosity (b) after type-inversion. 


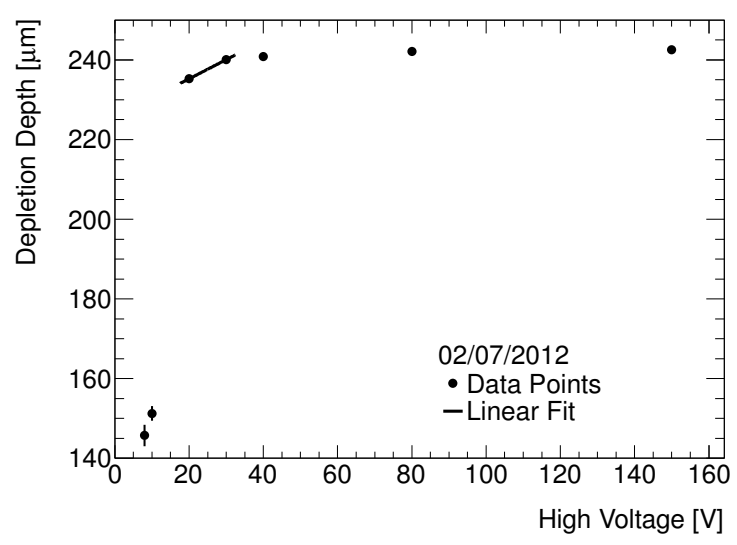

(a)

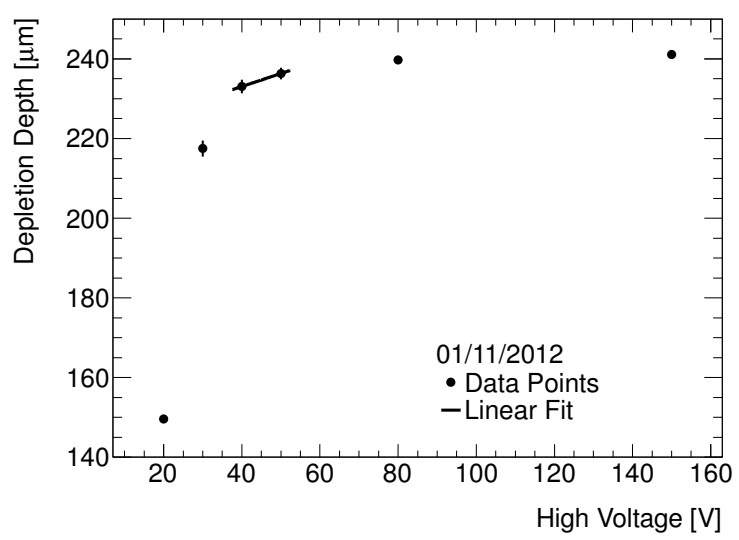

(c)

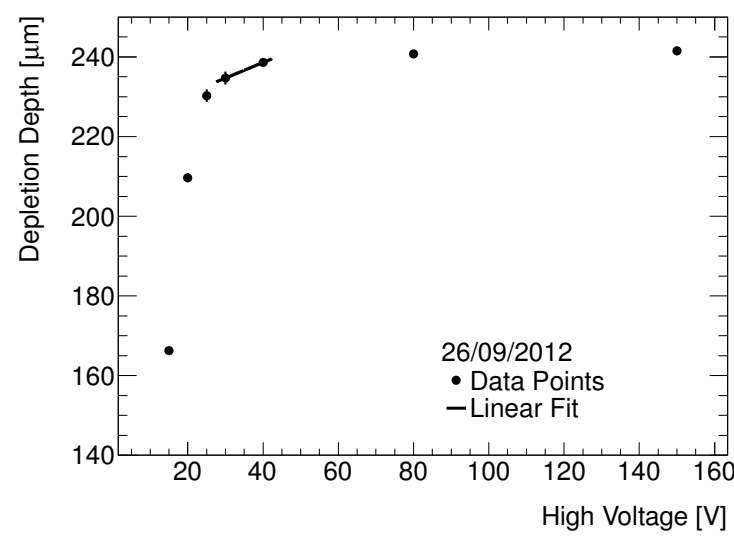

(b)

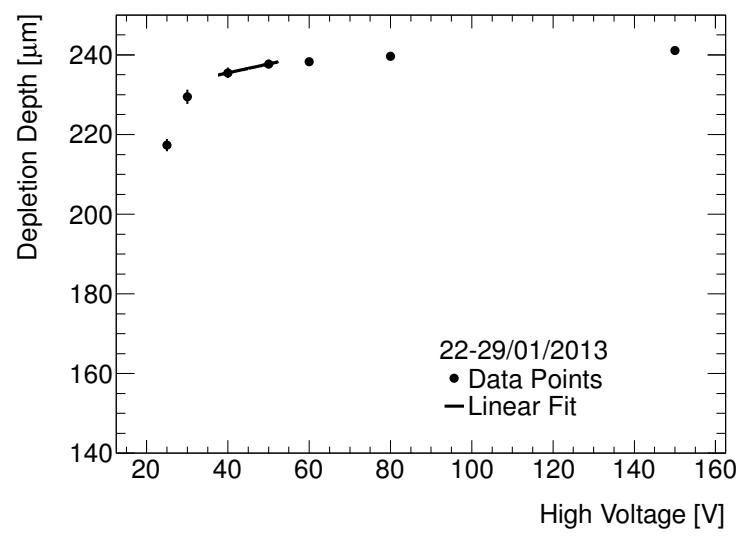

(d)

Figure A.6.: Mean value of depletion depth in Layer 0 shown as a function of the bias voltage. A linear fit is used to extract the effective depletion voltage. 


\section{B. Additional Tables}

\begin{tabular}{|c|c|c|}
\hline Time & Voltage & LumiBlock \\
\hline $07: 02$ & $150 \mathrm{~V}$ & $52-78$ \\
$07: 25$ & $80 \mathrm{~V}$ & $79-98$ \\
$07: 44$ & $40 \mathrm{~V}$ & $99-110$ \\
$07: 55$ & $30 \mathrm{~V}$ & $111-120$ \\
$08: 03$ & $20 \mathrm{~V}$ & $121-130$ \\
$08: 11$ & $10 \mathrm{~V}$ & $131-140$ \\
$08: 20$ & $8 \mathrm{~V}$ & $141-146$ \\
$08: 26$ & $6 \mathrm{~V}$ & $147-154$ \\
$08: 33$ & $150 \mathrm{~V}$ & $155-156$ \\
\hline
\end{tabular}

Table B.1.: Summary of depletion depth scan in layer 0 at the 02.07.2012. ATLAS Run 206253, LHC Fill 2797.

\begin{tabular}{|c|c|c|}
\hline Time & Voltage & LumiBlock \\
\hline $14: 03$ & $15 \mathrm{~V}$ & $62-73$ \\
$14: 08$ & $20 \mathrm{~V}$ & $74-86$ \\
$14: 13$ & $25 \mathrm{~V}$ & $87-98$ \\
$14: 18$ & $30 \mathrm{~V}$ & $99-119$ \\
$14: 24$ & $40 \mathrm{~V}$ & $120-133$ \\
$14: 30$ & $80 \mathrm{~V}$ & $134-146$ \\
$14: 35$ & $10 \mathrm{~V}$ & $147-159$ \\
$14: 40$ & $50 \mathrm{~V}$ & $160-171$ \\
\hline
\end{tabular}

Table B.2.: Second depletion depth scan in layer 0 at the 26.09.2012. ATLAS Run 211522, LHC Fill 3087.

\begin{tabular}{|c|c|c|}
\hline Time & Voltage & LumiBlock \\
\hline $11: 07$ & $10 \mathrm{~V}$ & $1260-1269$ \\
$11: 17$ & $40 \mathrm{~V}$ & $1270-1279$ \\
$11: 27$ & $80 \mathrm{~V}$ & $1283-1291$ \\
$11: 38$ & $30 \mathrm{~V}$ & $1292-1301$ \\
$11: 48$ & $20 \mathrm{~V}$ & $1302-1311$ \\
$11: 59$ & $50 \mathrm{~V}$ & $1312-1321$ \\
$12: 08$ & $150 \mathrm{~V}$ & $1322-1322$ \\
\hline
\end{tabular}

Table B.3.: Third depletion depth scan in layer 0 at the 01.11.2012. ATLAS Run 213754, LHC Fill 3249 . 


\begin{tabular}{|c|c|c|c|c|}
\hline Run & Date & Time & Voltage & LumiBlock \\
\hline 218036 & 22.01 .2013 & $23: 24$ & $30 \mathrm{~V}$ & $45-54$ \\
218036 & 22.01 .2013 & $23: 35$ & $40 \mathrm{~V}$ & $55-65$ \\
218036 & 22.01 .2013 & $23: 36$ & $50 \mathrm{~V}$ & $66-70$ \\
218168 & 24.01 .2013 & $22: 12$ & $80 \mathrm{~V}$ & $392-404$ \\
218168 & 24.01 .2013 & $22: 25$ & $60 \mathrm{~V}$ & $405-415$ \\
218168 & 24.01 .2013 & $22: 36$ & $50 \mathrm{~V}$ & $416-422$ \\
218179 & 25.01 .2013 & $10: 37$ & $25 \mathrm{~V}$ & $632-641$ \\
218179 & 25.01 .2013 & $10: 47$ & $30 \mathrm{~V}$ & $642-652$ \\
218436 & 29.01 .2013 & $09: 44$ & $50 \mathrm{~V}$ & $264-275$ \\
218436 & 29.01 .2013 & $09: 56$ & $40 \mathrm{~V}$ & $276-308$ \\
218436 & 29.01 .2013 & $10: 19$ & $150 \mathrm{~V}$ & $310-316$ \\
218436 & 29.01 .2013 & $10: 22$ & $30 \mathrm{~V}$ & $317-339$ \\
218436 & 29.01 .2013 & $10: 40$ & $150 \mathrm{~V}$ & $341-346$ \\
218436 & 29.01 .2013 & $10: 44$ & $40 \mathrm{~V}$ & $347-359$ \\
218436 & 29.01 .2013 & $10: 54$ & $50 \mathrm{~V}$ & $360-377$ \\
\hline
\end{tabular}

Table B.4.: Last depletion depth scan in layer 0 between the 22.01.2013 and the 29.012.2013.

\begin{tabular}{|c|c|c|}
\hline Time & Voltage & LumiBlock \\
\hline $12: 08$ & $10 \mathrm{~V}$ & $1323-1329$ \\
$12: 16$ & $40 \mathrm{~V}$ & $1330-1339$ \\
$12: 28$ & $15 \mathrm{~V}$ & $1342-1350$ \\
\hline
\end{tabular}

Table B.5.: First depletion depth scan in layer 1 at the 01.11.2012. ATLAS Run 213754, LHC Fill 3249.

\begin{tabular}{|c|c|c|}
\hline Time & Voltage & LumiBlock \\
\hline $10: 05$ & $20 \mathrm{~V}$ & $600-610$ \\
$10: 17$ & $30 \mathrm{~V}$ & $611-621$ \\
$10: 27$ & $40 \mathrm{~V}$ & $622-630$ \\
\hline
\end{tabular}

Table B.6.: Second depletion depth scan of layer 1 at the 2501.2013. ATLAS Run 218179, LHC Fill 3490. 


\section{Bibliography}

[1] J. Beringer et al. (Particle Data Group). Review of Particle Physics. Phys. Rev. D, 86:010001, 2012 and 2013 partial update for the 2014 edition.

[2] S.L. Glashow. Partial-symmetries of weak interactions. Nucl. Phys., 22:579-588, 1961.

[3] A. Salam and J.C. Ward. Electromagnetic and weak interactions. Phys. Lett., 13:168-171, 1964.

[4] S. Weinberg. A Model of Leptons. Phys. Rev. Lett., 19:1264-1266, 1967.

[5] F. Englert and R. Brout. Broken Symmetry and the Mass of Gauge Vector Mesons. Phys. Rev. Lett., 13:321-323, 1964.

[6] G.S. Guralnik, C.R. Hagen and T.W. Kibble. Global Conservation Laws and Massless Particles. Phys. Rev. Lett., 13:585-587, 1964.

[7] P.W. Higgs. Broken Symmetries and the Masses of Gauge Bosons. Phys. Rev. Lett., 13:508509,1964 .

[8] The ATLAS Collaboration. Observation of a new particle in the search for the Standard Model Higgs boson with the ATLAS detector at the LHC. Phys. Lett. B, 716:1-29, 2012.

[9] The CMS Collaboration. Observation of a new boson at a mass of $125 \mathrm{GeV}$ with the CMS experiment at the LHC. Phys. Lett. B, 716:30-61, 2012.

[10] P. Nason, S. Dawson and R.K. Ellis. The total cross section for the production of heavy quarks in hadronic collisions. Nucl. Phys. B, 303:607-633, 1988.

[11] R.K. Ellis, W.J. Stirling and B.R. Webber. QCD and Collider Physics. Cambridge University Press, 1996.

[12] The DELPHI Collaboration. A study of the b-quark fragmentation function with the DELPHI detector at LEP I and an averaged distribution obtained at the Z Pole. Eur. Phys. J. C, 71:1557, 2011.

[13] The ATLAS Collaboration. Physics at a High-Luminosity LHC with ATLAS. ATL-PHYSPUB-2012-001, 2012.

[14] The ATLAS Collaboration. Physics at a High-Luminosity LHC with ATLAS (Update). ATL-PHYS-PUB-2012-004, 2012.

[15] G. Lutz. Semiconductor Radiation Detectors. Springer, 1999.

[16] W. R. Leo. Techniques for Nuclear and Particle Physics Epxeriments. Springer, 1994.

[17] H. Bichsel. Straggling in thin silicon detectors. Rev. Mod. Phys., 60:663, 1988.

[18] L.D. Landau. On the energy loss of fast particles by ionization. J. Exp. Phys. (USSR), 8:201, 1944.

[19] P.V. Vavilov. Ionization Losses of High-Energy Heavy Particles. Sov. Phys. JETP, 5:749, 1957. 
[20] S. Hancock et al. Energy loss and energy straggling of protons and pions in the momentum range 0.7 to $115 \mathrm{GeV} /$ c. Phys. Rev. A, 28:615, 1983.

[21] S.M. Sze. Semiconductor Devices Physics and Technology. Wiley, second edition, 2002.

[22] L. Rossi et al. Pixel Detectors From Fundamentals to Applicstions. Springer, 2006.

[23] P.P. Altermatt et al. The influence of a new band gap narrowing model on measurements of the intrinsic carrier density in crystalline silicon. Int. Photovoltaic Sci. Eng. Conf., Sapporo, 11:719, 1999.

[24] Z. He. Review of the Shockley-Ramo theorem and its application in semiconductor gammaray detectors. Nucl. Instr. and Meth. in Phys. Res. A, 463:250-267, 2001.

[25] G. Kramberger and D. Contarato. Simulation of signal in irradiated silicon pixel detectors. Nucl. Instr. and Meth. in Phys. Res. A, 511:82-87, 2003.

[26] The ALICE Collaboration. The ALICE Experiment at the CERN LHC. JINST, 3:S08002, 2008.

[27] The ATLAS Collaboration. The ATLAS Experiment at the CERN Large Hadron Collider. JINST, 3:S08003, 2008.

[28] The CMS Collaboration. The CMS Experiment at the CERN LHC. JINST, 3:S08004, 2008.

[29] The LHCb Collaboration. The LHCb Detector at the LHC. JINST, 3:S08005, 2008.

[30] L. Evans and P. Bryant. LHC Machine. JINST, 3:S08001, 2008.

[31] J. Haffner. The CERN accelerator complex, 2013. General Photo.

[32] G. Bachy. Co-ordinate axis systems. CERN EDMS Note ATL-GE-CERN-QAP-0204.01, 1996.

[33] G. Aad et al. ATLAS Pixel Detector Electronics and Sensors. JINST, 3:P07007, 2008.

[34] D. Barberis et al. Pixel Detector Active Area Layout. CERN EDMS Note ATL-IP-EP0004, 2002.

[35] R. Beccherle et al. MCC: the Module Controller Chip for the ATLAS Pixel Detector. Nucl. Instr. and Meth. in Phys. Res. A, 492:117-133, 2002.

[36] J. Große-Knetter. Vertex Measurement at a Hadron Collider - The ATLAS Pixel Detector. Habilitation. BONN-IR-2008-04, Universität Bonn, 2008.

[37] I. Gorelov et al. A measurement of Lorentz angle and spatial resolution of radiation hard silicon pixel sensors. Nucl. Instr. and Meth. in Phys. Res. A, 481:204-221, 2002.

[38] T. Lari. Study of silicon pixel sensors for the ATLAS Detector. PhD thesis. CERNTHESIS-2001-028, Università degli studi di Milano, 2001.

[39] F. Hügging on behalf of the ATLAS Pixel Collaboration. Front-End electronics and integration of ATLAS pixel modules. Nucl. Instr. and Meth. in Phys. Res. A, 549:157-164, 2005.

[40] I. Perić et al. The FEI3 readout chip for the ATLAS pixel detector. Nucl. Instr. and Meth. in Phys. Res. A, 565:178-187, 2006.

[41] S. Welch and J. Dopke on behalf of the ATLAS Collaboration. The ATLAS Pixel nSQP Readout Chain. ATL-INDET-PROC-2012-018, 2012. 
[42] M.L. Chu et al. The off-detector opto-electronics for the optical links of the ATLAS Semiconductor Tracker and Pixel detector. Nucl. Instr. and Meth. in Phys. Res. A, 530:293$310,2004$.

[43] The ATLAS IBL Community. ATLAS Insertable B-Layer Technical Design Report. CERNLHCC-2010-013, 2010.

[44] The ATLAS IBL Community. ATLAS Insertable B-Layer Technical Design Report Addendum. CERN-LHCC-2012-009, 2012. Addendum to CERN-LHCC-2010-013.

[45] The ATLAS IBL Collaboration. Prototype ATLAS IBL modules using the FE-I4A frontend readout chip. JINST, 7:P11010, 2012.

[46] D.-L. Pohl on behalf on the ATLAS Collaboration. Overview of the ATLAS Insertable B-Layer (IBL) Project. ATL-INDET-PROC-2013-008, 2013.

[47] D. Muenstermann. R\&D on Planar Pixel Sensor Technology for the ATLAS Inner Detector Upgrade. CERN EDMS Note ATU-RD-MN-0029, 2008.

[48] G. Darbo C. Da Via S. Parker. Development of Full 3D Active-Edge and Modified 3D Silicon Pixel Sensors with Extreme Radiation Hardness. CERN EDMS Note ATL-PAMN-0007, 2006.

[49] M.S Alam et al. The ATLAS silicon pixel sensors. Nucl. Instr. and Meth. in Phys. Res. A, 456:217-232, 2001.

[50] C. Goessling et al. Evaluation of the breakdown behaviour of ATLAS silicon pixel sensors after partial guard-ring removal. Nucl. Instr. and Meth. in Phys. Res. A, 624:410-413, 2010 .

[51] C. Da Viá et al. Technical Specifications and Acceptance Criteria for the 3D Sensors of the ATLAS IBL. CERN EDMS Note ATU-SYS-QC-0004, 2011.

[52] P. Grenier et al. Test beam results of 3D silicon pixel sensors for the ATLAS upgrade. Nucl. Instr. and Meth. in Phys. Res. A, 638:33-40, 2011.

[53] The FE-I4 Collaboration. The FE-I4B Integrated Circuit Guide. Version 2.3, 2012.

[54] V.A.J. van Lint. Mechanisms of Radiation effects in Electronic Material. Wiley, 1980.

[55] G. Lindstöm. Radiation damage in silicon detectors. Nucl. Instr. and Meth. in Phys. Res. A, 512:30-43, 2003.

[56] R. Wunstorf. Systematische Untersuchungen zur Strahlenresistenz von Silizium-Detektoren für die Verwendung von Hochenergiephysik-Experimenten. PhD thesis. DESY-FH1K92-01, Universität Hamburg, 1992.

[57] M. Huhtinen. Simulation of non-ionising energy loss and defect formation in silicon. Nucl. Instr. and Meth. in Phys. Res. A, 491:194-215, 2002.

[58] A. Vasilescu and G. Lindström. Notes on the fluence normalisation based on the NIEL scaling hypothesis. ROSE/TN/2000-02, 2000.

[59] M. Moll et al. Relation between microscopic defects and macroscopic changes in silicon detector properties after hadron irradiation. Nucl. Instr. and Meth. in Phys. Res. B, 186:100$110,2002$.

[60] I. Pintilie et al. Second-order generation of point defects in gamma-irradiated float-zone silicon, an explanation for "type inversion". Applied physics letters, 82:2169-2171, 2003. 
[61] I. Pintilie et al. Radiation-induced point- and cluster-related defects with strong impact on damage properties of silicon detectors. Nucl. Instr. and Meth. in Phys. Res. A, 611:52-68, 2009.

[62] M. Moll. Radiation Damage in Silicon Particle Detectors - microscopic defects and macroscopic properties. PhD thesis. DESY-THESIS-1999-040, Universität Hamburg, 1999.

[63] G. Lindström et al. Developments for radiation hard silicon detectors by defect engineering-results by the CERN (RD48) ROSE Collaboration. Nucl. Instr. and Meth. in Phys. Res. A, 465:60-69, 2001.

[64] S. Haywood. Local Coordinate Frames for the Alignment of Silicon Detectors. ATLINDET-2004-001, 2004.

[65] F. Hirsch. B-Tagging calibration at the ATLAS experiment. PhD thesis. TU Dortmund, 2011.

[66] S. Gibson on behalf of the ATLAS Collaboration. Monitoring radiation damage in the ATLAS Silicon Tracker. PoS, RD11:035, 2011.

[67] A. L. Schorlemmer on behalf of the ATLAS Collaboration. Monitoring radiation damage in the ATLAS Pixel Detector. JINST, 8:C01045, 2013.

[68] The ATLAS Collaboration. ATLAS Approved Pixel Plots. https://twiki.cern.ch/ twiki/bin/view/AtlasPublic/ApprovedPlotsPixel and https://twiki.cern.ch/ twiki/bin/view/AtlasPublic/PixelPublicResults.

[69] G. Battistoni et al. The FLUKA code: description and benchmarking. AIP Conference Proceedings, 896(1):31-49, 2007. DOI: 10.1063/1.2720455.

[70] A. Ferrari et al. FLUKA: A multi-particle transport code (program version 2005). CERN2005-010, INFN-TC-2005-11, SLAC-R-773, 2005.

[71] O. Krasel. Charge collection in irradiated silicon-detectors. PhD thesis. TU Dortmund, 2004 .

[72] R. Klingenberg et al. Prediction of charge collection efficiency in hadron-irradiated pad and pixel silicon detectors. Nucl. Instr. and Meth. in Phys. Res. A, 568:34-40, 2006.

[73] I. Gorelov et al. A measurement of Lorentz angle and spatial resolution of radiation hard silicon pixel sensors. Nucl. Instr. and Meth. in Phys. Res. A, 481:204-221, 2002.

[74] A. L. Schorlemmer. Radiation damage assessment of ATLAS pixel sensors using continous depletion depths measurements. Diplomarbeit. TU Dortmund, 2009.

[75] G. Alimonti et al. A study of charge trapping in irradiated silicon with test beam data. ATL-INDET-2003-014, 2003.

[76] V. Eremin, E. Verbitskaya and Z. Li. Effect of radiation induced deep level traps on $\mathrm{Si}$ detector performance. Nucl. Instr. and Meth. in Phys. Res. A, 476:537-549, 2002.

[77] P. Behara et al. Threshold Tuning of the ATLAS Pixel Detector. ATL-INDET-PUB-2010001, 2010.

[78] The ATLAS Collaboration. Expected performance of the ATLAS experiment: detector, trigger and physics. CERN-OPEN-2008-20, 2009.

[79] N. G. Piacquadio. Identification of b-jets and investigation of the discovery potential of a Higgs boson in the $\mathrm{WH} \rightarrow l \nu \mathrm{b} \overline{\mathrm{b}}$ channel with the ATLAS experiment. PhD thesis. CERN-THESIS-2010-027, Universität Freiburg, 2010. 
[80] G. Piacquadio, K. Prokofiev and A. Wildauer. Primary vertex reconstruction in the ATLAS experiment at LHC. J. Phys.: Conf. Ser., 119:032033, 2008.

[81] M. Cacciari et al. The Anti-k(t) jet clustering algorithm. JHEP, 0804:063, 2008.

[82] T. Cornelissen et al. Concepts, Design and Implementation of the ATLAS New Tracking (NEWT). ATL-SOFT-PUB-2007-007, ATL-COM-SOFT-2007-002, 2007.

[83] The ATLAS Collaboration. Performance of the ATLAS Secondary Vertex b-tagging Algorithm in 7 TeV Collision Data. ATLAS-CONF-2010-042, 2010.

[84] The ATLAS Collaboration. Commissioning of the ATLAS high-performance b-tagging algorithms in the $7 \mathrm{TeV}$ collision data. ATLAS-CONF-2011-102, 2011.

[85] The ATLAS Collaboration. Measuring the b-tag efficiency in a top-pair sample with $4.7 \mathrm{fb}^{-} 1$ of data from the ATLAS detector. ATLAS-CONF-2012-097, 2012.

[86] T. Cornelissen et al. Updates of the ATLAS Tracking Event Data Model (Release 13). ATL-SOFT-PUB-2007-003. ATL-COM-SOFT-2007-008, 2007.

[87] The ATLAS Collaboration. Pile-up subtraction and suppression for jets in ATLAS. ATLAS-CONF-2013-083, 2013. 



\section{Acknowledgements}

My thesis would not have been possible without the help and support of many people during the past years. I want to thank everyone, since the group is too large for everyone to be mentioned.

First, I am very thankful to Arnulf Quadt for all of his guidance and of course for giving me the opportunity to write my thesis in Goettingen - I appreciate all of his help. I am also grateful to Joern Grosse-Knetter for all of his support.

I want to thank Beniamino Di Girolamo for all of his help and advice. He has always been very supportive and I learned a lot from him during my time at CERN. Furthermore, I want to thank Christoph Rembser, he has always been a great help and I am especially thankful for his encouragement in the past month, while I was writing my thesis. I am very thankful that I could spend the largest part of my Ph.D. at CERN within the Wolfgang-Gentner Program, and in that context I also want to thank Michael Hausschild who has always been very helpful.

I would like thank Jens Weingarten for all of all of his support, help, and guidance. I am especially thankful to him for proofreading my thesis.

Many thanks to Benjamin for his help, I really enjoyed working with him while he was at CERN in summer 2012.

Thanks to all of my colleagues at CERN with whom it was very nice to work. Special thanks to Stephen who always answered my questions, as well as Giacinto, Silvia and Kerstin.

Many thanks to Carla for our great time together and all of your encouragement and support.

Finally thanks to my family and all my friends, who have always supported me. 